\title{
COASTAL GROUNDWATER DYNAMICS - INVESTIGATION OF USING GEOELECTRICAL AND HYDROCHEMICAL TOOLS FOR SALINE INTRUSION MONITORING
}

BY

Eva MARIA SutTER

A THESIS SUBMITTED TO THE VICTORIA UNIVERSITY OF WELLINGTON IN FULFILMENT OF THE REQUIREMENTS FOR THE DegreE of Doctor of Philosophy

VICTORIA UNIVERSITY OF WELLINGTON 2018 



\section{Abstract}

With over $65 \%$ of the global population currently living in areas near a coast, increasing fresh groundwater demands within these areas, shifted precipitation patterns, and rising mean sea levels, increased seawater intrusion into coastal aquifers has become a major issue for groundwater resources in many coastal countries all around the world. Although there are many past studies researching the saline interface in affected aquifers from a modelling, laboratory or field perspective with different hydrological and geophysical approaches, little is known about real field dynamics over various time spans and in different geological settings. This PhD project aims at detecting and characterising seawater intrusion into a shallow coastal Holocene sand and gravel aquifer at New Zealand's west coast with respect to seasonally changing aquifer resistivity and hydrochemical tracers, as well as investigating resistivity and hydraulic property changes within a tidal time frame. Seawater mixing behaviour over different time spans was monitored with electrical resistivity tomography (ERT) over the course of two years, and additional hydrochemical sampling was carried out during the second year of the long-term seawater intrusion monitoring study. During two consecutive years, repeat ERT measurements were able to determine seasonal and shorter-term cycles in seawater mixing behaviour within the shallow coastal aquifer. There are strong indications that increasing urbanisation has a major influence on seasonal seawater intrusion patterns, and the dominant processes at the field locations were identified to be tied to the amount of freshwater available over the course of a year, as well as enhanced evapotranspiration / evaporation during summer. Hydrochemical data backed these observations and were also able to characterise seawater intrusion as a function of depth at the field location. Within the smaller tidal cycle investigations, similarly behaving parts of the aquifer could be identified from resistivity ratios and time series analyses. Varying amplitudes in resistivity changes led to the conclusion that saline mixing within a diurnal tidal cycle is strongly dependent on the recharge regime of the aquifer and decreases significantly for times during the year when recharge is continuous as opposed to times with little general recharge but intense, isolated rainfall events. In addition, tidal time series were used to qualitatively infer hydraulic properties of the aquifer and ultimately delineate preferential flow paths for seawater intrusion at the field site. The results of this project support findings of 
previous modelling, laboratory and short-term field studies, and put the concepts into a much broader time frame. For the first time the dynamics of seawater mixing in a shallow coastal sand aquifer were conclusively studied at the same location and within different time frames, and are thus of great value for sustainable groundwater management in the area of investigation and similar coastal environments. 
Dedicated to Harold.

Never stop believing in yourself.

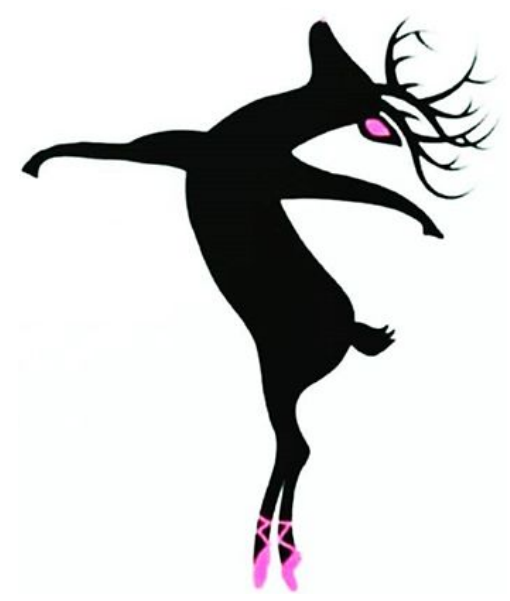





\section{Acknowledgements}

My special thanks go out to my primary supervisor Malcolm Ingham for his everlasting support throughout the entire process of this thesis with all its highs and lows, for his continuous approval of all the travels that I was able to do during the course of the project, for his endless encouragement and advice with the 'accursed chapter', and for all the good discussions (and laughters) many of the ideas in this thesis have thrived and prospered from. Thank you!

To my secondary supervisor Gideon Gouws for being an appreciated 'observer from the outside'. The thesis certainly benefited from his valuable comments to improve understanding for a non-expert in a very specialised field. And for his patience through the technical battles of each one of the six-monthly progress reports!

\section{"Knowledge is in the end based on acknowledgement."}

Ludwig Wittgenstein, philosopher (1889 - 1951)

For financial support through a doctoral scholarship I like to thank Victoria University of Wellington (VUW). Doug Mzila and Dave Turner from Greater Wellington Regional Council for their kind hospitality, suggestions of a suitable field area, access to their environmental monitoring data base and the gift of a (semi-functional) groundwater data logger.

My thanks extend to the field techs of SGEES at VUW Dez Tessler and Adrian Benson for lending me their equipment without hesitation and all the advice that came with it. Also, Nick Grinter and Manu Pouajen Blakiston from the mechanical workshop of SCPS at VUW for fulfilling all my mechanical desires for the field work ... yesterday! Thanks also to Peter Coard and David Stead from the electronics workshop at SCPS for their help, tools and advice when fabricating the borehole ERT equipment which sadly never got used.

The hydrochemical analysis of this thesis would not have been possible without the much valued assistance of Monica Handler. Thank you for letting me use the ICP-MS instrument, patiently explaining the data processing, and your opinion on the hydrochemistry chapter.

And then I would like to thank all the people who helped me acquiring the large amount of field data over the two years. Especially Celine Mandon and Jeff Welter who were always available to carry around heavy equipment in the sand dunes of Waikanae. I know you (and I) will never 
forget the 'pain-in-the-arse-profile'!

This is also a thank you to my friends for sharing stories and adventures, listening to the many rants, letting me weirdly try to explain what I do for a living and just being there to get my mind off the thesis. You know who you are! And last but certainly not of least importance, I would like to thank my family for their invaluable support and encouragement (mostly from far away) throughout these three years. Some of the 'doldrums' would definitely not have been that easy to handle if it were not for you guys. 


\section{Abstract}

Acknowledgements

List of Figures

vii

List of Tables

xvii

Abbreviations

xix

1 Introduction $\quad \mathbf{1}$

1.1 Coastal aquifers and their threat from seawater intrusion $\ldots \ldots \ldots \ldots$

1.2 Researching saltwater contamination processes - past and present . . . . . . . 5

1.3 Hydrogeophysics for saltwater intrusion characterisation . . . . . . . . . . . 10

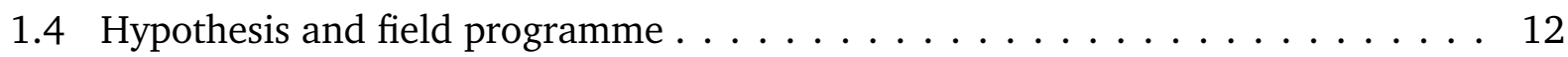

1.5 Objectives and structure of this thesis . . . . . . . . . . . . 17

2 The field area $\quad 21$

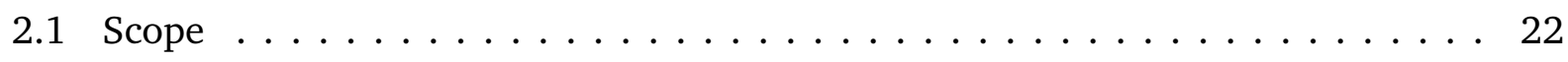

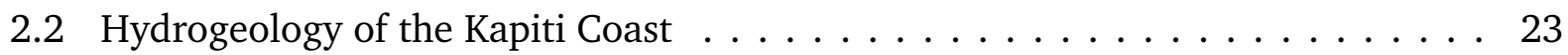

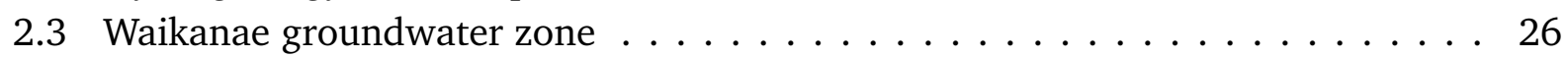

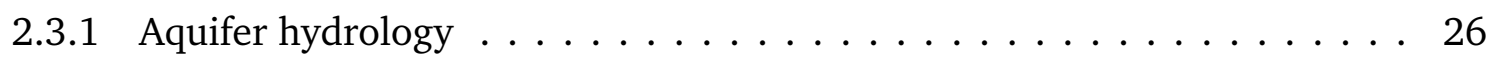

2.3 .2 Coastal geology . . . . . . . . . . . . . . . . . . . 29

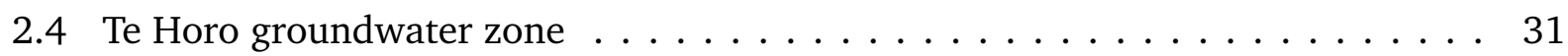

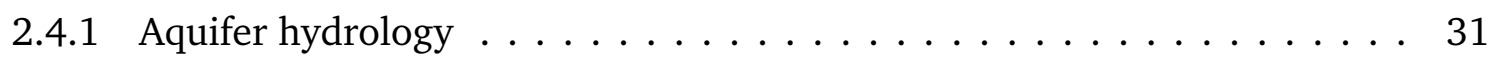

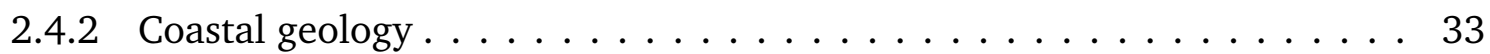

3 Methodologies $\quad 37$

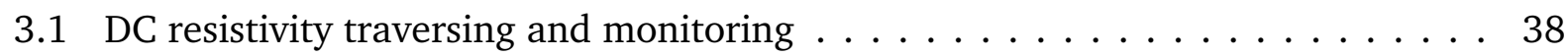

3.2 Geophysical inversion for time-lapse monitoring data sets . . . . . . . . . . . . . . . . . . 43

3.2.1 Data editing and error estimation . . . . . . . . . . . . . 43

3.2 .2 Producing time-lapse inversion models . . . . . . . . . . . . . 44

3.2.3 Inversion model appraisal - the depth-of-investigation method . . . . . . 47

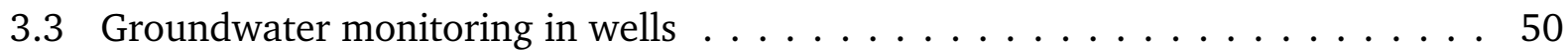

3.4 Chemical analysis of groundwater . . . . . . . . . . . . 52 
3.4 .1 Hydrochemistry through ICP-MS . . . . . . . . . . . . . . . . . . . 53

3.5 Dynamic Time Warping for measuring signal time lags . . . . . . . . . . . . . . . . . . . . 57

3.6 Autocovariance for periodicity retrieval in time series . . . . . . . . . . . . . 59

4 Seasonal time-lapse geoelectrical monitoring of salinity changes in shallow coastal aquifers

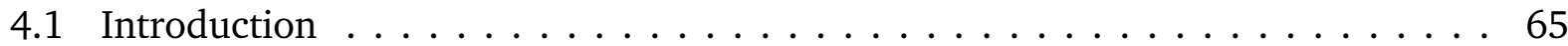

4.2 Field sites and geoelectrical measurements $\ldots \ldots \ldots \ldots$

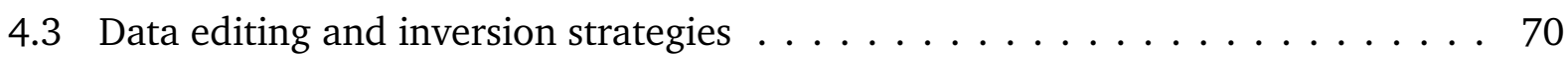

4.4 Monitoring results from two groundwater zones . . . . . . . . . . . . . . 72

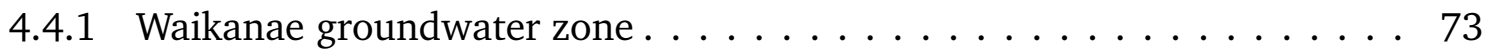

4.4 .2 Te Horo groundwater zone . . . . . . . . . . . . . . . . . 79

4.5 Discussion of seasonal seawater intrusion effects on a shallow coastal aquifer . . 85

4.6 Summary . . . . . . . . . . . . . . . . . . 90

5 The role of the tides - analysis of tidal geoelectrical time series for periodic seawater mixing

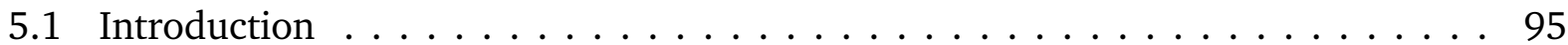

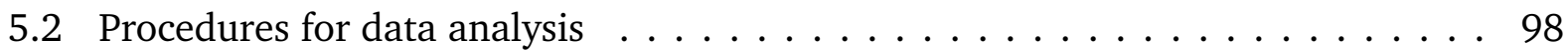

5.2 .1 Time-lapse DC resistivity filtering and inversion . . . . . . . . . . . . . . . 98

5.2.2 On displaying long $2 \mathrm{D}$ geoelectrical time-lapse series . . . . . . . . . . 100

5.2.3 Inferring hydraulic ground properties with statistical methods . . . . . . . 101

5.3 Diurnal tidal cycle monitoring in the Waikanae groundwater zone . . . . . . . 105

5.3.1 Tidal resistivity ratios and time series . . . . . . . . . . . . . . 105

5.3.2 Warping costs and cycle times - or: Is the resistivity signal dependent on the tides? . . . . . . . . . . . . . . . . . . . . . . . . . 114

5.4 Summary . . . . . . . . . . . . . . . . . . . . . 120

6 Combining groundwater chemistry and DC resistivity for characterisation of sea$\begin{array}{ll}\text { sonal seawater intrusion dynamics } & 123\end{array}$

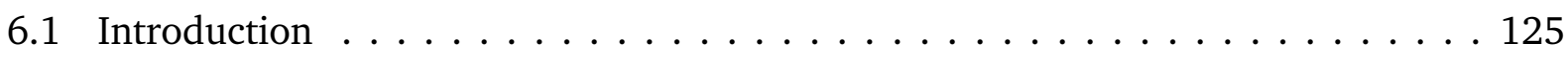

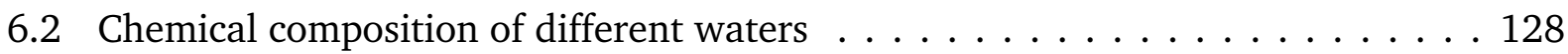

6.3 Field procedures and data analysis . . . . . . . . . . . . . . 130

6.3 .1 Hydrochemical data . . . . . . . . . . . . . . . . . . . . . . . . . . . . . . . . . . . . . . . . . . . . . .

6.3 .2 Geoelectrical data . . . . . . . . . . . . . . . . . . . 135

6.4 Hydrochemical monitoring results and their implications . . . . . . . . . . . . 136

6.4.1 Electric conductivity, total dissolved solids and salinity relationships . . . 138

6.4.2 Continuous electric conductivity, temperature and water level bore logging148

6.4.3 Estimating hydraulic conductivity from tidal amplitude attenuation . . . 151

6.5 Geoelectrical monitoring results and their implications . . . . . . . . . . . . 152

6.6 Discussion and insights from combining time-lapse hydrochemical and geoelectrical data . . . . . . . . . . . . . . . . . . . . . . . . 155

6.7 Long-term implications for seawater intrusion in Te Horo Beach . . . . . . . . . 161

6.8 Summary . . . . . . . . . . . . . . . . . . . 166 


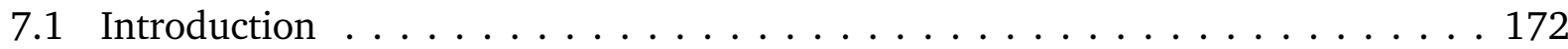

7.2 A conceptual model of fresh- and saltwater dynamics in the shallow groundwater aquifer at the Kapiti Coast . . . . . . . . . . . . . . . . . . . . . . . . 173

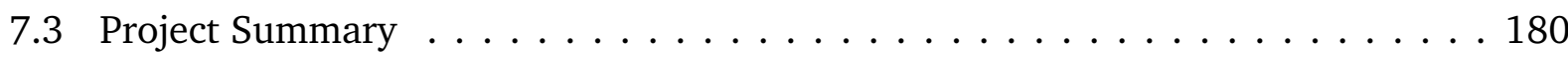

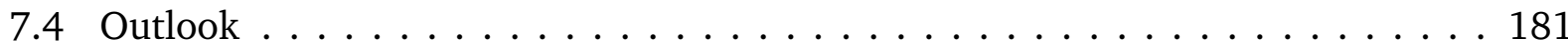

7.4 .1 Geoelectrical methods . . . . . . . . . . . . . . . . . . . . . . 181

7.4 .2 Hydrochemical methods . . . . . . . . . . . . . . . . . 182

7.4 .3 Time series methods . . . . . . . . . . . . . . . . . . . . 183

7.4 .4 Other recommendations . . . . . . . . . . . . . . . . 184

A 3D DC resistivity study of the saline interface shape 201

B Saline mixing front velocity $\quad 205$

C Seasonal implications from repeated tidal resistivity monitoring 209

C.1 Introduction to the Te Horo tidal monitoring data sets . . . . . . . . . . . . . . 209

C.2 The tide's seasonality - implications from resistivity ratios and time series . . . 211

C.3 Summary . . . . . . . . . . . . . . . . . . . . . . . 214

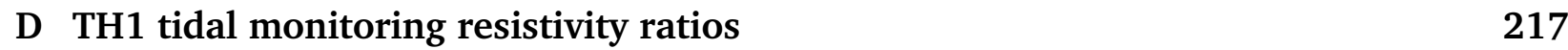

E Tidal DTW modelling for a better understanding of field data 225

F Estimate of resistivity periods from well groundwater table data 231

G Groundwater chemistry - $\mathrm{pH}$ and dissolved oxygen relationships 235

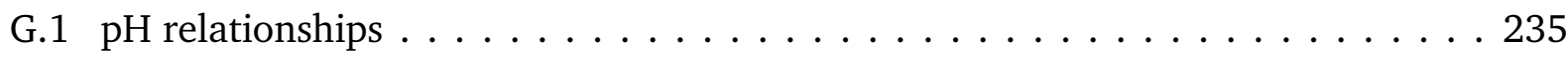

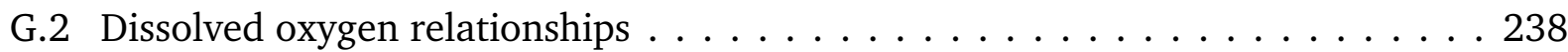

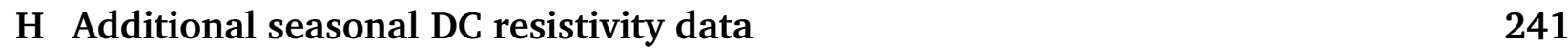

I YSI sensor results and ICP-MS analyses $\quad 249$ 



\section{List of Figures}

1.1 Simple model for the saline interface predicted by the Ghyben-Herzberg formulation. A sharp boundary between freshwater and saltwater is assumed, with a freshwater discharge point located at the beach face (zero freshwater head). The equation $z=\left(\rho_{f} /\left(\rho_{s}-\rho_{f}\right)\right) * h$ predicts the saline interface depth $z$ underneath the freshwater head $h$ relative to the mean sea level for two fluid densities $\rho$ (freshwater and saline water). . . . . . . . . . . . . . .

1.2 Conceptual model of main hypothesis. . . . . . . . . . . . . . . . . . 13

1.3 Long term (15 years) EC sampling in well R26/6587 (GWRC monitoring data base) about $3 \mathrm{~km}$ away from the coast line. Orange segments mark warmer months (summer), whereas the blue segments highlight those where temperatures are lower (winter). . . . . . . . . . . . . . . . . .

2.1 Map of the field area (Kapiti Coast) in New Zealand showing the Waikanae/Paraparaumu (referred to as Waikanae field area due to being located in the Waikanae groundwater zone) and Te Horo field areas. The Tararua Mountain Range where most of the groundwater recharge happens, runs SW-NE in the eastern part of the study area. The regional council measures groundwater stage (amongst others) in wells R26/6287 and R26/6886, and at the Te Hapua wetland. Rainfall data is obtained from the water treatment plant and the Te Hapua wetland. Tidal stage information comes from NIWA for the Waikanae- and Otaki River mouths. . . . .

2.2 Schematic illustration of the Waikanae groundwater zone with the six hydrostratigraphic units (Q1-Q6), their estimated hydraulic conductivity and indicated preferential flow paths as suggested by Utting (2013) and Gyopari et al. (2014). The figure has been modified from Gyopari et al. (2014). . . . . . . . . . . . .

2.3 Correlation between groundwater table height near the coast and precipitation records from the Tararua foot range. a) Groundwater table level data for the Waikanae DC resistivity monitoring period measured in well R26/6287 (GWRC monitoring network). Dots mark the acquisition dates of resistivity data on the N2 profile (see Fig. 4.1). b) Hourly rainfall data for the DC resistivity monitoring period as recorded at the Waikanae Water Treatment Plant (locations see Fig. 2.1). 29

2.4 Bore logs obtained for three wells drilled around the Waikanae Beach area. Their location is indicated in Fig. 2.1. R26/6566 and R26/6541 information was adapted from (Morrow, 2007), whereas the one for R26/6806 was taken from (Osborne, 2006). Note that the depth is measured in meters above mean sea level. 30 
2.5 Geologic cross section drawn from bore information from three wells marked orange in Fig. 2.1 in the northern part of Waikanae. A more detailed location of these wells and the geological profile line is given in Fig. 4.1 (also in orange

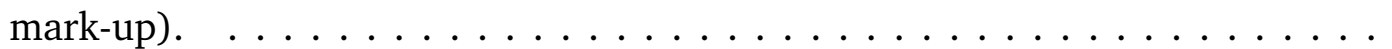

2.6 Schematic illustration of the Te Horo groundwater zone with the six hydrostratigraphic units (Q1-Q6), their estimated hydraulic conductivity and indicated preferential flow paths as suggested by Utting (2013) and Gyopari et al. (2014). The figure has been modified from Gyopari et al. (2014). . . . . . . . . . . . . . .

2.7 Groundwater stage and rainfall at the Te Hapua Wetland monitoring station (see Fig. 2.1) in Te Horo Beach (GWRC monitoring network) for the DC resistivity monitoring period in Te Horo. Red dots indicate sampling dates for the seasonal monitoring of profile THS1 (see Fig. 4.2) . . . . . . . . . . . . . .

2.8 Bore logs obtained for two wells drilled in Te Horo Beach. Their location is indicated in Fig. 2.1 (a close-up of their location can be found in Fig. 4.2). Note that the depth is measured in meters above mean sea level.

2.9 Geologic cross section adapted from (Wilson, 2003). Depths are in meters above mean sea level. Horizontal distances are in meters. Bore locations are marked

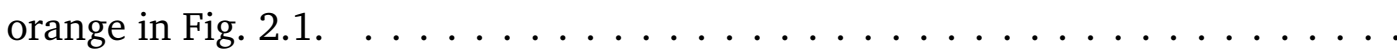

3.1 Basic illustration of a current flowing in the subsurface between two current electrodes ( $\mathrm{C} 1$ and $\mathrm{C} 2$ ) placed at the surface. Associated potential lines cut the current lines in a $90^{\circ}$ angle. At geophysical boundaries the current lines are bent and refracted and hence different subsurface conditions excite different potential signals measured at the surface through potential electrodes (P1 and P2). . . .

3.2 An example of how DOI works. a) calculated scaled DOI index for the entire model space, $b$ ) the extended inversion model using the average resistivity of the field data as a background model, c) histogram and fitted probability density function (PDF) (for each Gaussian distribution) for the cut-off factor analysis, and d) the scaled inversion model that is blending out unreliable model parts as defined by the scaled DOI index and the statistically determined cut-off factor (note: if the DOI index values would be only distributed unimodally (i.e. only one Gaussian distribution of DOI values) then a cut-off factor would need to be chosen manually). . . . . . . . . . . . . . . . . . . . . . . .

3.3 Schematic of an ICP-MS system. The sample gets vaporized into the argon plasma (created by a radio frequency coil that produces oscillating electromagnetic fields), where it goes from an atomic into an ionic state. At the interface between the ICP and the MS systems, the positively charged ions travel through interface cones that are connected by a vacuum chamber in order to allow for pressure differences between the two instrument parts. An electrostatic filter (e.g. quadrupole mass filter or sector field filter) only allows to pass ions with a specific mass-to-charge ratio which is defined by the measurement sequence and changes over time so all elements of interest can be measured. Then the filtered ions get counted through the intensity of the electric signal in the detector they excite (proportional to the number of ions in the sample).

3.4 Difference of similarity (distance) measure between a) cross-correlation of two similar signals (blue and red) shifted in time, and b) dynamic time warping (DTW) of two similar signals with different temporal behaviour. . . . . . . . . . 
3.5 Cost matrix D with optimal warping path (blue) for a dynamic time warping (DTW) analysis of the tidal stage signal (X) and a model cell conductivity time series $(\mathrm{Y})$. The red rectangle marks cell $\mathrm{D}(\mathrm{N}, \mathrm{M})$ which represents the overall similarity/separation of the two signals. . . . . . . . . . . . .

3.6 Auto-covariogram of a 21-hour tidal signal at the field site in Te Horo. It becomes clear that the zero-amplitude crossings mark the time between the tidal extremes every six hours (no correlation of the signal with itself), whereas positive correlation is achieved approximately every twelve hours (i.e. the period of the signal). Negative correlation occurs roughly six hours after a positive correlation (i.e. low vs. high tide stage) and its recurrence time is also about twelve hours. . . .

4.1 Map of the study area around Waikanae (Kapiti Coast (NZ)). Indicated in red are the six DC resistivity transects, blue points mark groundwater table level a GWRC monitoring well and the Waikanae water treatment plant, whereas orange points show where the geologic bore log information was obtained. AA' marks the geologic section drawn from the bore information (Fig. 2.5). . . . . . . . .

4.2 Map of the study area around Te Horo Beach (Kapiti Coast (NZ)). Indicated in red are the three $155 \mathrm{~m}$ long DC resistivity transects that were monitored in a roughly 30-day cycle during 2015/2016. North of the settlement the Mangaone Stream is discharging into the sea. . . . . . . . . . . . . . .

4.3 The resistivity equipment as set up in the field (shown here for a $2-\mathrm{m}$ spaced tidal measurement). On the left side regular $0.35 \mathrm{~m}$ long stainless steel electrodes are connected to the 8-core cable take outs (every $5 \mathrm{~m}$ ) with elastic O-rings. Up to six cables were connected (64 channels) for some of the longest transects. A $12 \mathrm{~V}$ deep cycle battery was used to power the ARES resistivity meter. In the right picture the set up on the $1.2 \mathrm{~m}$ long electrodes is imaged. This was used at the beach to avoid seawater contamination of the equipment there (note, this is shown for a $2 \mathrm{~m}$ minimum spacing survey). . . . . . . . . . . .

4.4 (a) Time-lapse inversion models of DC resistivity data along traverse N2 (Fig. 4.1) with superimposed $45-\Omega \mathrm{m}$ contour lines and (b) corresponding resistivity ratios between subsequent time steps with contour lines indicating a resistivity ratio of 1.0. Wavy lines in the western part of the absolute resistivity plots in (a) indicate the seawater level during measurement.

4.5 Resistivity ratios of the DCR time-lapse inversion models for all six profiles (north to south order) between August 2014 and February 2015 ( $\mathrm{R}=$ Feb/Aug). The images were scaled with the DOI index method (opacity level), and the contour lines mark a resistivity ratio of 1.0 (no change) between the two time steps investigated. Water levels dropped in average by $355 \mathrm{~mm}$ in well R26/6287 (see Fig. 4.1) between the August and February acquisition dates of the profiles. .

4.6 Resistivity ratios of the DCR time-lapse inversion models for all six profiles (north to south order) between February 2015 and June 2015 ( $\mathrm{R}=\mathrm{Jun} / \mathrm{Feb}$ ). The images were scaled with the DOI index method (opacity level), and the contour lines mark a resistivity ratio of 1.0 (no change) between the two time steps investigated. Water levels increased in average by $636 \mathrm{~mm}$ in well R26/6287 (see Fig. 4.1) between the February and June acquisition dates of the profiles. . 
4.7 (a) Time-lapse inversion models of DC resistivity data along traverse TH1 (Fig. 4.2) with superimposed $45-\Omega \mathrm{m}$ contour lines and (b) corresponding resistivity ratios between subsequent time steps with contour lines indicating a resistivity ratio of 1.0. The wave run-up during measurements only reached the electrodes in the January and August 2016 surveys (up to the second electrode at $5 \mathrm{~m}$ profile extent). . . . . . . . . . . . . . . . . . . . .

4.8 Resistivity ratios of the DCR time-lapse inversion models for all three profiles (north to south order) between October 2015 and February 2016 (R = Feb/Oct), where the February data set taken for the ratio calculation was the one previous to the rain event. Water levels decreased in average by $395 \mathrm{~mm}$ at the Te Hapua wetland monitoring site (GWRC monitoring network (location see Fig. 2.1)) between the October and February acquisition dates of the profiles. The letter $B$ marks the increase in resistivity of the conductive middle layer. . . . . . . . . .

4.9 Resistivity ratios of the DCR time-lapse inversion models for all three profiles (north to south order) between February 2016 and May 2016 ( $\mathrm{R}=$ May/Feb), where the February data set taken for the ratio calculation was the one previous to the rain event. Water levels increased in average by $124 \mathrm{~mm}$ at the Te Hapua wetland monitoring site (see Fig. 2.1) between the February and May acquisition dates of the profiles. The letter $B$ marks the decrease in resistivity of the conductive middle layer. $\ldots \ldots \ldots \ldots \ldots \ldots \ldots \ldots$

4.10 Resistivity ratios of the DCR time-lapse inversion models for all three profiles (north to south order) between February 2016 and August 2016 (R = Aug/Feb), where the February data set taken for the ratio calculation was the one previous to the rain event. Water levels increased in average by $437 \mathrm{~mm}$ at the Te Hapua wetland monitoring site (see Fig. 2.1) between the February and August acquisition dates of the profiles. The letter $B$ marks the decrease in resistivity of the

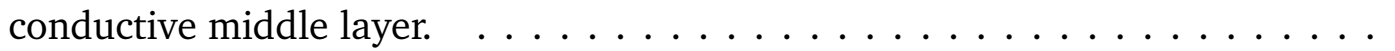

4.11 Sample aquifer model to illustrate how different processes can lead to unexpected behavior in seasonal time-lapse geoelectrical models as observed along urbanely located profiles in this study. . . . . . . . . . . . . .

5.1 Two examples of two sinusoidal signals at a time shift with a) the same frequency (period) and b) different frequencies. It becomes quickly clear that the time lag between the signals in (a) is always constant, while the time lag between the signals in (b) differs by large amounts depending on what part of the time series is examined.

5.2 Example plot of long time series. Each model cell element shows the amplitude (i.e. resistivity) on the y-axis, and the time on the x-axis. All of them are then plotted in the model space, illustrating the portions of the profile that show similar temporal behaviour. Note, model resistivity scales differ for each cell.

5.3 Example of the effects of transforming and smoothing time series. Black curves are the original data of the time series (note difference in scale on the right yaxes), in blue the transformed signals, and in red the transformed and smoothed signals. Note that the scale for the latter two is the same and displayed on the left y-axes. Data was taken for a) tidal time series, b) an inversion model cell with a decreasing then increasing conductivity trend, and c) an inversion cell with an increasing then decreasing conductivity trend. . . . . . . . . . . . . . 104 
5.4 a) Inversion models for the 21-hour time series collected in November 2015 along profile N2 in the Waikanae groundwater zone (see Fig. 4.1), and b) the resistivity ratios between each consecutive time step. . . . . . . . . . . . . . 107

5.5 N2 21-hour resistivity ratios between subsequent high and low tides (top: LT1/• HT1, bottom: HT2/LT1). Aquifer parts that show an increase of resistivity with decreasing tidal stage and a decrease of resistivity with increasing tidal stage are marked with $A$, whereas parts marked $B$ show the opposite behaviour. . . . . . 108

5.6 N2 21-hour time series shown as resistivity time series in a) (i.e. each model cell has time on the $\mathrm{x}$-axis and resistivity amplitude on the y-axis), and coloured according to their temporal behaviour in b) (i.e. same colour equals same temporal behaviour of the resistivity). This allows to display the entirety of Fig. 5.4 in just one (or two) figure(s). Note that there is no topography included

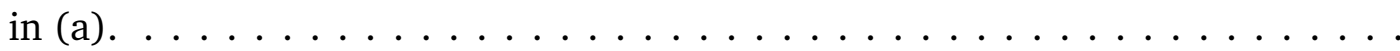

5.7 The five characteristic curve shapes identified in the Waikanae N2 inversion model time series. 1) bell-shape, 2) U-shape, 3) fluctuating, 4) L-shape, and 5) J-shape. 6) shows the tidal stage signal during the DC resistivity monitoring at the field site for reference. . . . . . . . . . . . . . . . . . . . . 110

5.8 Water movement (perforated arrows) for the situations of a a) falling (four hours before high tide to low tide) and b) rising (low tide to three hours after high tide) tidal stage within the aquifer as indicated by changes in the $10 \%(6 \Omega \mathrm{m}), 1$ $\%(45 \Omega \mathrm{m})$ and $0 \%(140 \Omega \mathrm{m})$ seawater mixing contours from the 21 time-lapse inversion models of the DC resistivity monitoring in Waikanae. Red and blue solid arrows indicate relative hypothetical flow direction of seawater and freshwater, respectively. Black arrows mark the direction of the contour lines with increasing

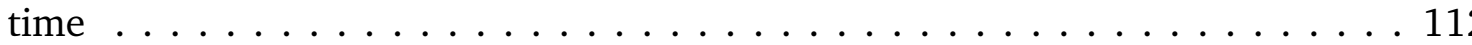

5.9 DTW between a) tidal stage and resistivity, and b) groundwater stage and resistivity signals of each individual model cell of the 21-hour tidal monitoring along the Waikanae N2 profile. Letters $A$ are associated with resistivity decrease during tidal stage increase in previous plots, whereas letters $B$ are marking parts of the aquifer showing a resistivity increase with increasing tidal stage. . . . . . . . . 116

5.10 Seven-day time series and FFT derived periods of tidal stage at the Waikanae River mouth (black), groundwater levels in wells R26/6287 (dark blue) and R26/6886 (light blue), and atmospheric pressure at Moa Point in Wellington (red).118

5.11 Autocovariance results of each model cell of the 21-hour tidal monitoring timelapse inversion along profile N2 in Waikanae. Model elements that exceed a period of 21 hours, are regarded not containing any seawater (i.e. resistivity $>$ $140 \Omega \mathrm{m}$ ), have a resistivity range within the time series that is below $5 \Omega \mathrm{m}$ or show unreasonable cycle times, are blanked for interpretational reasons. . . . . . .

6.1 Map of the Te Horo Beach settlement with the Mangaone Stream to its north. Green dots indicate bore sampling locations where YSI sensor and ICP-MS hydrochemical data are available for all three sampling dates, while blue dots mark those where groundwater sampling results from February and August 2016 are available. Red dots indicate the location of bores that were only tested once during the sampling period. Long-term electrical conductivity (EC), groundwater stage and temperature monitoring was performed in the bore marked with the orange dot $(R)$. Geoelectrical monitoring was done along profile $\mathrm{TH} 2_{\text {ext }}$. . . . 
6.2 a) Groundwater sampling in the field. The multi-probe sensor in the bucket determines parameters like EC, TDS, salinity, temperature and dissolved oxygen of the continuously running groundwater. Once the parameters stabilize it can be assumed that the water is representative of the extraction depth and the groundwater sample can be taken. b) Sample filtering in the laboratory in order to remove solid particles before acidification process. c) Dilution of the samples with $1 \%$ nitric acid. Actual weights of the dilution are measured and noted in order to calculate an accurate dilution factor for concentration recovery after ICP-MS measurements. . . . . . . . . . . . . . . . . .

6.3 Groundwater stage (top) and rainfall (bottom) measured at the Te Hapua wetland GWRC environmental monitoring network station (location see Fig. 2.1) from May 2015 until September 2016. Blue dots indicate the times of the groundwater sampling, and red the ones of the resistivity measurement campaigns, respectively. 137

6.4 Total dissolved solids (TDS) vs. electrical conductivity (EC) (left), and TDS vs. salinity (right) measured in Te Horo Beach bores. Blue dots represent the two winter measurements, whereas pink ones are from the summer sampling. . . . . 139

6.5 Temperature difference vs. TDS difference of all bore samples and between subsequent samplings (red dots = abs(winter 2015-summer 2016), green dots $=\mathrm{abs}$ (summer 2016-winter 2016)). The temperature variation is generally low between seasons and there is no notable trend visible with TDS. Only bore \# 8 exceeds a temperature difference of $5-10{ }^{\circ} \mathrm{C}$, which is considered the limit for temperature influence of TDS by Nelson (2002). . . . . . . . . . . . . . . . . . 141

6.6 Electric conductivity plotted against the four major cations ( $\mathrm{Na}, \mathrm{Mg}, \mathrm{Ca}$ and $\mathrm{K}$ ). A positive linear trend can be found for $\mathrm{Na}, \mathrm{Mg}$ and $\mathrm{Ca}$, but is not evident in $\mathrm{K}$. Increasing concentrations in the major elements have a significant influence on EC as they account for the main TDS content in groundwater. . . . . . . . . . . 142

6.7 Major element ratios shown for $\mathrm{Na} / \mathrm{K}, \mathrm{Na} / \mathrm{Ca}$ and $\mathrm{Mg} / \mathrm{Ca}$. The plotted trend lines indicate the ratios of the relative major cations for average data measured in the Mangaone stream (M) representing freshwater, the seawater (S) west of the sampling site, and a theoretical $1 \%$ mixture of fresh- and seawater at this location (Fig. 6.1). . . . . . . . . . . . . . . . . . . . . . . . 143

6.8 Trace metals for which data were available (reliable) are plotted against their associated EC measurements. Some of them could only be detected in summer (Al) or winter $(\mathrm{Cr}$ and $\mathrm{Pb})$. Their concentrations are generally low and seasonal variations in barium are marginal. For reference, the values of the trace elements are also plotted for the Mangone stream samples (M) . . . . . . . . . . . . . . . . 146

6.9 Seawater related ( $\mathrm{Li}$ and $\mathrm{Sr}$ ) and non-seawater related (Fe and $\mathrm{Mn}$ ) trace metals plotted against salinity. Data quality is generally better for $\mathrm{Li}$ and $\mathrm{Sr}$ as a result of detectability. Positive relationships of the measured concentrations for trace metals in groundwater samples with salinity are apparent for lithium and strontium.

6.10 a) groundwater EC and temperature, and b) converted ( $=1 / \mathrm{EC})$ groundwater resistivity and groundwater stage, continuously monitored in bore R (Fig. 6.1) between December 2015 and August 2016. 
6.11 DC resistivity models and ratios made on three occasions (winter 2015, summer 2016 and winter 2016) on transect $\mathrm{TH} 2_{\text {ext }}$. Numbers 1 to 4 refer to the four features identified in the resistivity models in Chapter 4 and are discussed in the text. Black lines on the resistivity ratio images roughly outline the model parts showing low resistivity.

6.12 Sampled bores coloured according to their bore depth with respect to the mean sea level. Green $=$ above mean sea level, orange $=$ between 0 and $5 \mathrm{~m}$ below mean sea level, and pink = deeper than $5 \mathrm{~m}$ below mean sea level. The two sampling locations $\mathrm{S}$ and $\mathrm{M}$ are surface waters and are hence not appointed a depth category. . . . . . . . . . . . . . . . . . . . 156

6.13 Sampled bores coloured according to their potential for seawater mixing. Pink dots show a tendency for seawater mixing in two or more of the measured parameters. Green dots indicate that there was little or no implication for seawater mixing. Blue dots mark bores that likely tap into old seawater lenses or stagnant old groundwater that is enriched in TDS. With pale coloured dots bores are marked for which only one sample was taken during the sampling period to indicate which behaviour in terms of seawater mixing it indicates based on that

6.14 Resistivity model (along extended TH2 profile in Fig. 6.1) and to bulk resistivity transformed bore water EC measurements (dots with error bars) of the February 2016 groundwater sampling campaign. Both use the same colour scale. Bore numbers as assigned in Chapter 6 are given with bold black numbers and the bore water bulk resistivity values are stated as grey numbers (in $\Omega \mathrm{m}$ ). The absolute values of the two methods agree well when superimposed and can be used to constrain each other. . . . . . . . . . . . . . . .

6.15 Resistivity and EC ratios superimposed on each other for the extended profile TH2 in Te Horo (Fig. 6.1). Bore numbers are indicated in bold, whereas the groundwater EC ratios are stated with light numbers. Note that the colour scale is the same for the hydrological and geophysical data. The results of the two methods show good agreement within errors. . . . . . . . . . . . . . . 160

6.16 Te Horo Beach properties existing as indicated from the Google Earth 2005 satellite image (black) versus the one of the 2016 update (green). Each square marks one house. . . . . . . . . . . . . . . . . . . 162

6.17 Comparison of three studies that measured electrical conductivity in bores around the Te Horo Beach settlement. Data for January 1998 are from Hughes (1998), those for April 2003 were taken from Wilson (2003), and those of February 2016 are from the current groundwater study (see also Chapter 6) . . . . . . . . . . . 164

6.18 Comparison of the three studies in Te Horo Beach measuring EC vs. distance from coast. Colours refer to EC ranges used in Fig. 6.17 (blue $=200-400 \mu \mathrm{S} / \mathrm{cm}$, green $=400-600 \mu \mathrm{S} / \mathrm{cm}$, orange $=600-800 \mu \mathrm{S} / \mathrm{cm}$, pink $=>800 \mu \mathrm{S} / \mathrm{cm})$. Note that errors on April 2003 data may be up to $100 \mu \mathrm{S} / \mathrm{cm}$, whereas those from January 1998 and February 2016 are in the order of $1 \mu \mathrm{S} / \mathrm{cm} . \ldots \ldots$. . . . 165 
6.19 TH1 profile measured in January 2003 for the study carried out by Wilson (2003) and the same profile measured in January 2016 from the Te Horo Beach seasonal monitoring data set (13 years later). The contour lines indicate the $45 \Omega \mathrm{m}$ isoline which is considered about $1 \%$ saltwater mixing (as introduced in Chapter 4). At the bottom the resistivity ratio between the two inversion models is shown for model cells that exist in both data sets. . . . . . . . . . . . . . . . . . . .

7.1 Conceptual model of the Kapiti Coast shallow aquifer within about $1 \mathrm{~km}$ from the shoreline including all relevant processes of the groundwater system discussed in this thesis. The coastal mixing zone illustrated in the grey rectangle is imaged in more detail in Fig. 7.2. . . . . . . . . . . . . . . . . . . . . . . 175

7.2 Zoom into the mixing zone of the shallow coastal aquifer conceptual model in Fig. 7.1 showing the relevant processes that are taking place in this zone. . . . 178

A.1 Map of additional geoelectrical surveys in Te Horo Beach. The $\mathrm{TH} 1_{3 D}$ survey was done once in order to get a better understanding of the saline interface shape. P1-P6 mark six profiles perpendicular to the TH1 transect. . . . . . . . . . . . . . . 201

A.2 Vertical slice along the TH1 profile line of the 3D inverted DC resistivity data from a measurement made along TH1 and six perpendicular transects (P1-P6) at the north-western end of TH1 in February 2016. Shaded contours indicate sensitivity where values below 0.6 were regarded having no model reliability. . . 202

A.3 a) Horizontal slices at three depth levels through the 3D model that was produced from the resistivity data collected along the TH1 profile and the six additional transects perpendicular to it. b) Sensitivity maps of the models at the three depth levels shown in (a). Note that areas in the models (a) with a sensitivity (b) of below 0.6 (blue to yellow) are considered unreliable. . . . . . . . . . . .

C.1 Groundwater stage (top) and rainfall (bottom) measured at the Te Hapua wetland GWRC environmental monitoring network station (location see Fig. 2.1) from September 2015 until October 2016. Red dots indicate the times of the DC resistivity tidal monitoring dates along profile $\mathrm{TH} 1 . \ldots \ldots \ldots \ldots \ldots$

C.2 Resistivity ratios between high and low tide (or between time steps 6 hours apart) for all of the six tidal monitorings carried out along the Te Horo Beach TH1 profile (see Fig. 4.2). Letters $A$ refer again to parts of the aquifer that react in the expected way with decreasing tide, whereas letters $B$ mark those areas that behave in the opposite way. . . . . . . . . . . . . . . . . . 213

D.1 Resistivity ratios between each consecutive time step for the November 2015 tidal monitoring along the $\mathrm{TH} 1$ profile . . . . . . . . . . . . . . . . 218

D.2 Resistivity ratios between each consecutive time step for the February 2016 tidal monitoring along the $\mathrm{TH} 1$ profile . . . . . . . . . . . . . . . . . . . . . 219

D.3 Resistivity ratios between each consecutive time step for the April 2016 tidal monitoring along the $\mathrm{TH} 1$ profile . . . . . . . . . . . . . . . . . 220

D.4 Resistivity ratios between each consecutive time step for the June 2016 tidal monitoring along the $\mathrm{TH} 1$ profile $\ldots \ldots \ldots \ldots 21 \ldots \ldots \ldots 21$

D.5 Resistivity ratios between each consecutive time step for the first (small tidal range) September 2016 tidal monitoring along the TH1 profile . . . . . . . . . . 
D.6 Resistivity ratios between each consecutive time step for the first (large tidal range) September 2016 tidal monitoring along the $\mathrm{TH} 1$ profile . . . . . . . . . . .

E.1 Modelling of possible relationship between DC resistivity and tidal signals with a) two typical DC resistivity model cells for parts $A$ and $B$ plotted together with the tidal stage which approximate to about b) their sinusoidal representation from which a period of 20 hours for the DC resistivity signal was estimated, and used in c) to model electrical conductivity of those cells in order to find the same relationship between the three signals from d) which shows the signals for tidal stage with a period of 12 hours and the two inverse resistivity signals (i.e. conductivity) for $A$ and $B$ with periods of 20 hours and a time shift of 7 and 17 hours, respectively. However, as e) and d) show, there are several possibilities in a long time series for the time lags to occur in different variations. . . . . . . . . .

F.1 Normalised groundwater table stage obtained from well $R$ (Fig. 6.1) in Te Horo Beach (blue) and tidal stage at Otaki River mouth (Fig. 2.1) (black) for two monitoring periods in December 2015 and February 2016 (168 hours, 7 days). Note the different units on the y-axis. . . . . . . . . . . . . . . . . 232

F.2 Signal periods obtained from Fourier transforms for the groundwater (blue) and tidal (black) stage imaged in Fig. F.1 during two different times of the year. While the tidal stage shows a clear maximum amplitude at around 12 hours, the groundwater stage appears to have a main period of 21 hours. . . . . . . . . . .

G.1 Temperature vs. $\mathrm{pH}$ for all bores and samplings. A negative relationship is found between decreasing temperature and increasing $\mathrm{pH}$. . . . . . . . . . . 236

G.2 pH of bore samples taken three times between June 2015 and August 2016 in the Te Horo Beach township plotted against the major cations magnesium and calcium. The $\mathrm{pH}$ shows seasonal dependency for all bores. . . . . . . . . . . . 23

G.3 Manganese in relation to $\mathrm{pH}$ for groundwater samples taken in winter 2015. From this it seems as decreasing $\mathrm{pH}$ conditions lead to exponentially increasing Mn concentrations in the shallow aquifer. However, note that there is no $\mathrm{Mn}$ data available for other samplings which would be necessary to reliably confirm such a behaviour. . . . . . . . . . . . . . . . . 238

G.4 Dissolved oxygen (DO) in relation to temperature and $\mathrm{pH}$ for all measurements carried out in the sampling period. Green dots mark summer (s) and winter (w) values for measurements taken in the seawater (S) and Mangaone stream (M)

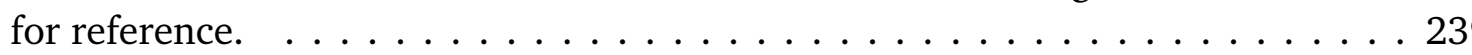

H.1 (a) Time-lapse inversion models of DC resistivity data along traverse N1 (Fig. 4.1) with superimposed $45-\Omega \mathrm{m}$ contour lines and (b) corresponding resistivity ratios between subsequent time steps with contour lines indicating a resistivity ratio of

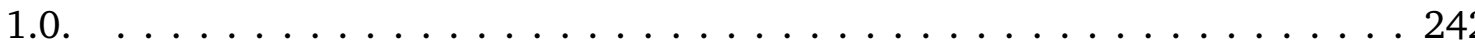

H.2 (a) Time-lapse inversion models of DC resistivity data along traverse N3 (Fig. 4.1) with superimposed $45-\Omega \mathrm{m}$ contour lines and (b) corresponding resistivity ratios between subsequent time steps with contour lines indicating a resistivity ratio of

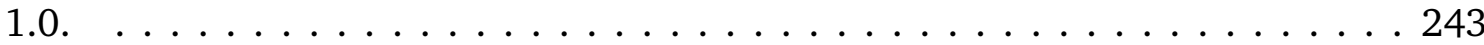


H.3 (a) Time-lapse inversion models of DC resistivity data along traverse R1 (Fig. 4.1) with superimposed $45-\Omega \mathrm{m}$ contour lines and (b) corresponding resistivity ratios between subsequent time steps with contour lines indicating a resistivity ratio of

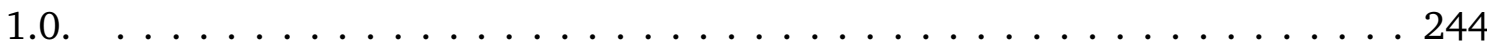

H.4 (a) Time-lapse inversion models of DC resistivity data along traverse T1 (Fig. 4.1) with superimposed $45-\Omega \mathrm{m}$ contour lines and (b) corresponding resistivity ratios between subsequent time steps with contour lines indicating a resistivity ratio of

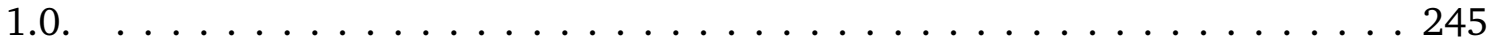

H.5 (a) Time-lapse inversion models of DC resistivity data along traverse S1 (Fig. 4.1) with superimposed $45-\Omega \mathrm{m}$ contour lines and (b) corresponding resistivity ratios between subsequent time steps with contour lines indicating a resistivity ratio of

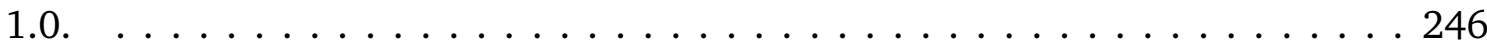

H.6 (a) Time-lapse inversion models of DC resistivity data along traverse TH2 (Fig. 4.2) with superimposed $45-\Omega \mathrm{m}$ contour lines and (b) corresponding resistivity ratios between subsequent time steps with contour lines indicating a resistivity ratio of

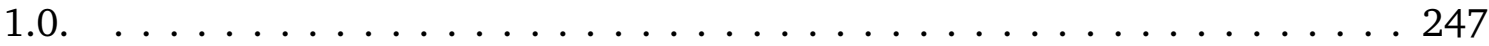

H.7 (a) Time-lapse inversion models of DC resistivity data along traverse TH3 (Fig. 4.2) with superimposed $45-\Omega \mathrm{m}$ contour lines and (b) corresponding resistivity ratios between subsequent time steps with contour lines indicating a resistivity ratio of

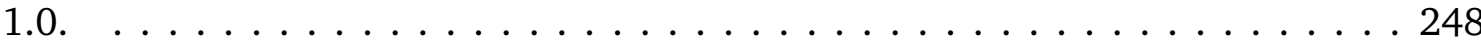




\section{List of Tables}

2.1 Hydrostratigraphic units and their description for the aquifers found along the Kapiti Coast (modified from Gyopari et al. (2014)). Not all units are present everywhere along the coast, as indicated for example in Fig. 2.2. . . . . . . . . 25

3.1 Groundwater monitoring parameters used in this thesis and how they are measured along with general resolution and accuracy information (compilation from Western et al. (2005); YSI Incorporated (2009) and Schlumberger Water Services (2010)). Underlined methods were used in this thesis and the resolution and accuracy stated are related to these methods. . . . . . . . . . . . . . . . .

4.1 Survey parameters for the two DC resistivity monitoring sites. The positions of the monitoring profiles are indicated in Figs. 4.1 and 4.2 for the Waikanae and the Te Horo groundwater zones, respectively. . . . . . . . . . . . .

6.1 Average composition and concentrations of major cations in seawater and river water. All concentrations are given in $\mathrm{mg} / \mathrm{l}$. Ratios between different cations can be used to identify the source of a water sample. The last column provides hypothetical values of a $1 \%$ mixing of seawater with river water which is considered to be enough to render freshwater undrinkable (Werner et al., 2013). Seawater intrusion should therefore be indicated by the changed ratios, especially those involving sodium.

6.2 Measured parameters in this thesis and their guideline values as stated in Ministry of Health New Zealand (2008) (black) or WHO (2011) (blue) if there was no statement made in the DWSNZ. The last column states the main reasons for measuring these particular parameters. This will be discussed in more detail in Section 6.4 however. . . . . . . . . . . . . . . . . . . . . 130

6.3 Bore depth information for the 18 bores that were sampled for groundwater at least once during the one-year period (see Fig. 6.1 for locations). Coordinates are given in NZ Map Grid.

6.4 Bore categories according to their electrical conductivity, bore depth and bore characteristic. Bracketed bores were only measured once during the survey. . . 140

6.5 Trace element concentrations for average seawater at 35 ppt salinity as stated by Chester (2000). . . . . . . . . . . . . . . . . . . . . . . . . 145

6.6 Groundwater stage in well R25/0003 on Sims Road in Te Horo Beach (GWRC monitoring network) for the three electrical conductivity surveys. Note that these are average values for the time spans given in 'Sampling Date'. . . . . . . . . 163 
B.1 Average saline mixing front velocities derived from three different isolines and their temporal expansion. The column value refers to the isoline resistivity plus indication of horizontal or vertical velocity estimate or ratio of these two velocity estimates. The error estimate for the velocity of each profile is given on the line following the profile name in percentage and underneath each measurement in absolute values. . . . . . . . . . . . . . . . . . . . . . . 207

C.1 Tidal range at Otaki river mouth and groundwater level at Te Hapua wetland for the tidal monitoring acquisition dates along the Te Horo Beach TH1 profile.

E.1 Optimal warping cost differences for different monitoring window lengths of two modelled resistivity signals with a period of 12 hours and actual time shifts of 2 and 8 hours with respect to a tidal signal with at 12 hour period. Standard deviations and mean values were calculated from DTW cost differences of each window shifted through a five-day signal length in one-hour steps. . . . . . . . . 226

I.1 YSI sensor measurements for bores 2, 4, 5, 7, 8, 9, 11, 15, 17, 18 and the Mangaone stream. . . . . . . . . . . . . . . 250

I.2 YSI sensor measurements for bores $1,3,6,10,12,13,14,16$ and the seawater. 251

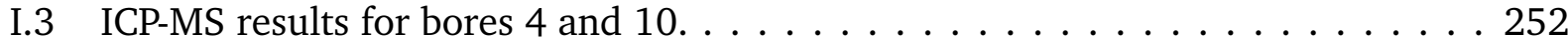

I.4 ICP-MS results for bores 7, 8, 17 and the Mangaone stream. . . . . . . . . . . . . . . . . . . . . . . . . . . . . . .

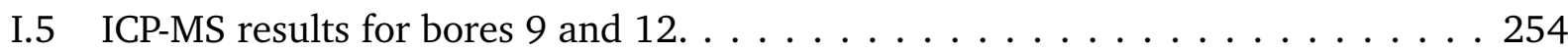

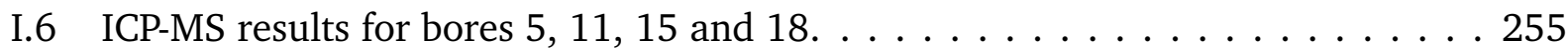

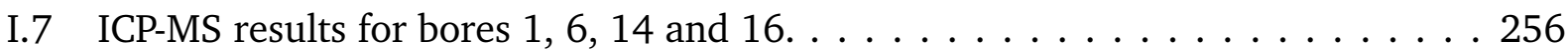

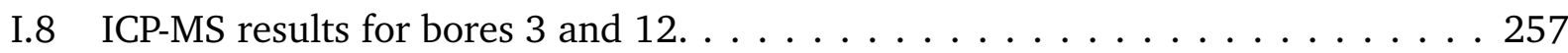

I.9 ICP-MS results for bores 13 and $R$, and the seawater. . . . . . . . . . . . 258 


\begin{tabular}{ll} 
ALERT & Automated Time-Lapse Electrical Resistivity Tomography \\
DC & Direct Current \\
DCR & Direct Current Resistivity \\
DO & Dissolved Oxygen \\
DOI & Depth Of Investigation \\
DTW & Dynamic Time Warping \\
DWSNZ & Drinking Water Standards for New Zealand \\
EC & Electrical Conductivity \\
ERT & Electrical Resistivity Tomography \\
GV & Guideline Value \\
GQSoE & Groundwater Quality State of Environment programme \\
GWRC & Greater Wellington Regional Council \\
GWSoE & Groundwater State of Environment programme \\
IAEA & International Atomic Energy Agency \\
ICP-MS & Inductively Coupled Plasma Mass Spectrometry \\
IDL & Instrument Detection Limit \\
KCDC & Kapit Coast District Council \\
MAV & Maximum Acceptable Value \\
NIWA & National Institute of Water and Atmospheric research \\
NMR & Nuclear Magnetic Resonance \\
PE & Polyethylene \\
PES & Polyethersulfone \\
PP & Polypropylene \\
RF & Radio Frequency \\
\hline
\end{tabular}


RMSE Root-Mean-Squared Error

SD Standard Deviation

SI Seawater Intrusion

SGD Submarine Groundwater Discharge

SWIM Sea Water Intrusion Meeting

TDS Total Dissolved Solids

UNESCO United Nations Educational, Scientific and Cultural Organization

WHO World Health Organization 


\section{CHAPTER 1}

\section{Introduction}

"The secret of getting ahead is getting started."

Mark Twain, writer (1835 - 1910)

In this introduction I give an overview of the investigated problem - saline intrusion into coastal aquifers - as well as a summary of how this phenomenon was and is studied in hydrology and hydrogeophysics. I then introduce the hypothesis and field programme, and provide an outline of the thesis structure. 


\subsection{Coastal aquifers and their threat from seawater intru- sion}

For as long as there has been life on this planet, freshwater has been needed for survival. Freshwater only makes up about $3 \%$ of all water on Earth (97\% is saltwater) (Oude Essink, 2001), but most organisms depend on a source of clean water. It is therefore important to make sure these sources are not contaminated and made undrinkable. Of this small amount of freshwater (35 x 10 $\mathrm{km}^{3}$ (Oude Essink, 2001)) compared to the vast amount of saltwater that is not directly available for consumption, more than two thirds is stored in the ice caps of the Arctic and Antarctic, as ice in permafrost regions and in the form of glaciers. Almost one third of all freshwater is stored underground in so called aquifers, which therefore makes up by far the largest resource of liquid freshwater. Less than one percent of all drinking water is available at the surface in rivers, streams and lakes (Oude Essink, 2001). Although taking freshwater for supply purposes from surface sources seems to be a logical thing to do, these sources are not accessible everywhere and are also often subject to contamination by local agricultural and industrial runoff. Groundwater on the other hand offers a vast storage of freshwater that is much better protected from direct infiltration of contaminants (filtration of water through soil and rock). This type of freshwater is stored in aquifers made up of different rocks and sediments which provide some sort of pore space that is able to accommodate water.

A major source of freshwater is provided by shallow sand and gravel aquifers which are found all around the coastal areas and make up approximately $25 \%$ of the worldwide coast line (Custodio, 1987). These are typically areas of high population (currently about two thirds of the entire global population lives within $60 \mathrm{~km}$ of the coast (McGranahan et al., 2007)) due to a large abundance of food and generally good economic prosperity (Oude Essink, 2001). Demands from domestic, agricultural and industrial sides are putting the aquifers of these regions under high supply stress. Although groundwater is generally well protected against direct contamination, it is still vulnerable through several threats posed to coastal (and other) aquifers that arise from natural and anthropogenic processes. In this thesis I put a focus on the threat due to seawater intrusion (SI). A good overview of other possible threats to coastal aquifers can be found in Custodio (1987) or Oude Essink (2001). 
A coastal aquifer typically has a boundary to the seawater at one end resulting in a zone of mixed fresh- and seawater called the transition or mixing zone that defines the saline interface. This zone is of variable width depending on hydrological and geological conditions. In early studies by Badon Ghyben (1889) and Herzberg (1901) the interface was described as a static and sharp boundary where salt- and freshwater were clearly separated. This simplification is commonly known as the Ghyben-Herzberg principle and was, and still is, widely applied to model the saline interface in coastal aquifer problems. It is based on the difference in density of the two fluids caused by the difference in their salinity, suggesting that in a stable system the freshwater 'floats' above the denser seawater with a landward sloping boundary (Fig. 1.1). Unstable conditions can for example occur below a wide tidal zone, near the shoreline if the freshwater discharge is below the sea level, or in coastal zones that are affected by sea storms or sea spray (Custodio, 1987). These are situations where denser seawater overlies freshwater and are quite common under natural conditions. However, apart from the Ghyben-Herzberg model having significant limitations itself (Custodio, 1987), field and modelling studies suggest that the transition zone between fresh- and seawater makes up a non-negligible part of the aquifer and represents a highly dynamic system with many parameters influencing its extent and equilibrium (e.g. Custodio, 1987; Werner et al., 2013). In the mixing zone salt concentration and fluid density undergo constant variation which is driven by mechanical dispersion and molecular diffusion and these processes control convection within the mixing zone wedge and together with flow velocity also the thickness of the transition zone (Werner et al., 2013). There are other processes involved in the control of this dynamic system such as heterogeneity in geology (and hence differing hydraulic properties such as hydraulic conductivity and permeability), tidal and wave forcing, temporal variability in groundwater level, long-term changes in sea level position, and anthropogenic pumping activities (Werner et al., 2013), many of which are important possible contributors to SI. These processes are often neglected in simplified models, however, they may be major driving forces of SI at different spatial and temporal scales. To date it is not fully understood in detail how these processes contribute exactly to the interplay of fresh- and seawater at their boundary. Although not the only concern when it comes to coastal aquifer characterisation, one of the main tasks for groundwater management is to estimate the position and extent of the saline mixing zone. 


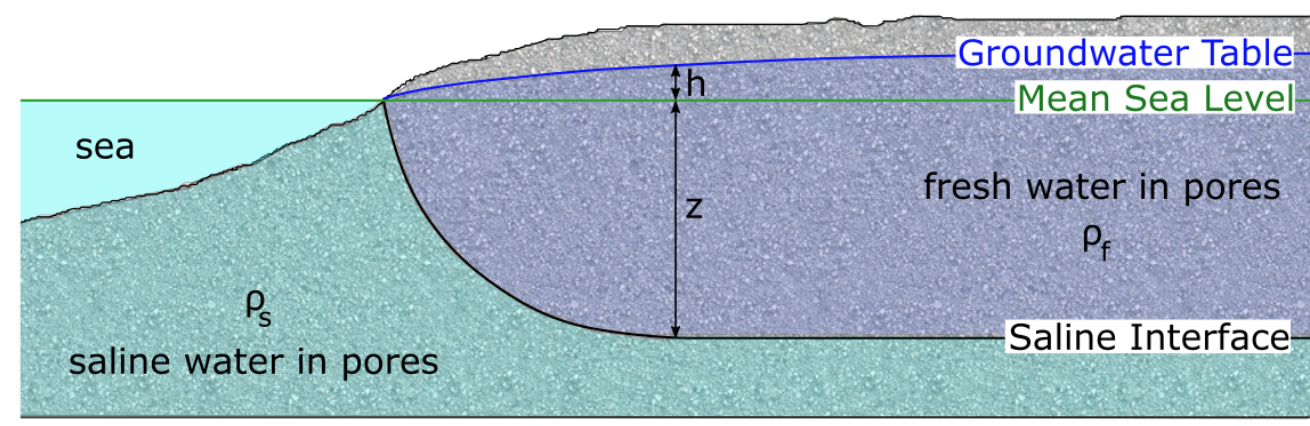

FIGURE 1.1: Simple model for the saline interface predicted by the Ghyben-Herzberg formulation. A sharp boundary between freshwater and saltwater is assumed, with a freshwater discharge point located at the beach face (zero freshwater head). The equation $z=\left(\rho_{f} /\left(\rho_{s}-\rho_{f}\right)\right) * h$ predicts the saline interface depth $z$ underneath the freshwater head $h$ relative to the mean sea level for two fluid densities $\rho$ (freshwater and saline water).

Seawater is a typical contamination source and the main source of salt in a coastal aquifer (Custodio, 1987). Contamination occurs in the form of subsurface replacement of freshwater with saltwater and often is referred to as seawater intrusion. Relative sea level rise caused by land subsidence, tectonic uplift or melting of the global ice masses is one of the reasons why seawater intrusion can occur. In a general and simplified scenario, the boundary between the two fluids is shifted if there is a change in their pressure relationship. Lowered pressure from the freshwater side will allow seawater to enter the aquifer further inland, whereas an increase in freshwater pressure opposes this process. A change in the pressure regime within an aquifer can be due to several reasons, possibly the most important of which are changes to the hydrological regime such as changes in recharge, precipitation and evapotranspiration rates (Oude Essink, 2001). These processes are controlled mainly naturally, but with increasing focus of groundwater as a freshwater source (Barlow, 2003), human activities can also be important to changes in the hydrologic regime. Anthropogenic influences on an aquifer system are associated with domestic and commercial groundwater extraction, land remediation, mining, and lowering of the groundwater level due to civil engineering structures (Custodio, 1987). With a special focus on coastal aquifers saltwater intrusion can also be influenced by shoreline erosion or retreat, and tidal effects (Werner et al., 2013; Oude Essink, 2001). These are all processes that influence the relative position of the freshwater-seawater interface and lead to changes in both the saline interface position and mixing zone thickness, thereby essentially defining how much saltwater is allowed to intrude the coastal aquifer at any time and altering the dynamic equilibrium. Additionally, there may be saltwater intrusion threats from surface sources such as rivers and estuaries by saline surface water seepage (Oude Essink, 2001) or severe sea storms. 
It does not take much seawater ( $<1 \%$ Werner et al., 2013) to render freshwater unfit for drinking purposes and only about 3-5 \% to make it unsuitable for irrigation (Custodio, 1987). Once a well or arable soil is contaminated it is a long-term, expensive and difficult task to remediate the consequences of SI. It therefore needs to be priority to avoid seawater contamination and in order to do so a good understanding of the processes that lead to SI is necessary. Economic management of the groundwater resources in coastal aquifers is therefore a challenging task as the above discussed processes basically provide infinite different possibilities for SI to occur and it is important to accurately identify the major driving force of SI at each individual site. Although many past and present research studies have contributed towards revealing the mechanisms of saltwater intrusion, the processes influencing, and the dynamics related to, the phenomena are still not clearly understood. Extensive field studies and the combination of different methods and research fields are essential to gain an understanding of the processes involved in SI and the rate of salinity change. The past and present work involved with this is discussed in the following sections.

\subsection{Researching saltwater contamination processes - past and present}

Since 1965 the United Nations Educational, Scientific and Cultural Organization (UNESCO) has put a substantial effort into understanding hydrological principles. This has led to a vast improvement of knowledge about the water resources on this planet, and thereby essentially introduced the profession of the modern hydrologist (Custodio, 1987). The existence of a fluid boundary within a coastal aquifer was already recognised at the turn of the last century (Badon Ghyben, 1889; Herzberg, 1901), but has not been considered an important aspect of groundwater hydrology until the late 1950's (Custodio, 1987). So called Sea Water Intrusion Meetings (SWIM), where saltwater intrusion is discussed on various scales and across several disciplines, have been convened in Europe since the late 1960's. Possibly the earliest hydrology literature discussing seawater intrusion into coastal aquifers were written by Todd (1958) and Cooper et al. (1964). In 1987 Custodio (1987) identified increasing freshwater demand as a problem for the quantity and quality of the natural groundwater resources in coastal areas and 
stressed the need for a global policy of sustainable water resource management. This was the first comprehensive evaluation of the impact of groundwater extraction on the saline interface in coastal areas and can be regarded as good summary of saltwater intrusion processes (Werner et al., 2013).

First attempts at characterising submarine groundwater discharge (SGD) (SGD is basically the opposite of SI) were made by using groundwater balance calculations. However, these were considered unreliable due to the large accumulation of errors and the estimating character of the models (Custodio, 1987). Analytical modelling of the relationships between fresh- and saltwater in an aquifer and how these relationships change depending on the various parameters (i.e. flow velocity, fluid density, hydraulic parameters of the aquifer) evolved practically parallel to these first groundwater balance calculations and still find applications nowadays (e.g. Glover, 1959; Henry, 1964). Most models used the sharp interface approach proposed by the Ghyben-Herzberg principle along with other assumptions and boundary conditions in order to be able to solve the governing equations analytically. Because in real situations aquifer properties change in space and groundwater flow, recharge and discharge in both space and time, analytical solutions became impractical and numerical models were looked for (Custodio, 1987). In the 1970's numerical models that use finite difference and finite element methods to transform differential equations and boundary conditions into algebraic equations and use iterative approaches to solve groundwater problems, soon gained popularity with the evolution of improved computational capacity. They allowed for relatively fast and cheap computation of $2 \mathrm{D}$ representations of different aquifer configurations and changes thereof, but were (and still are) subject to accurate field information without which the non-calibrated models essentially only represent one of many possibilities of the true situation (Custodio, 1987).

A parallel development was the use since the mid 1960's of observation wells for gaining salinity and electrical conductivity logs (e.g. Kohout, 1964; Todd and Meyer, 1971; Cotecchia, 1977) from which changes during different hydrologic conditions could be observed directly in the field for the first time. Along with these observations came measurements of groundwater chemistry which were used to produce for example scatter and Piper diagrams, isotope ratios and chemical hydrograms. With their aid the groundwater in coastal areas was classified and maps of seawater encroachment could be produced (e.g. Custodio, 1967; Beck and Hanshaw, 
1970; Tadolini and Tulipano, 1975). Chemical analyses require sampling of groundwater from a well and hence both field methods were (and still are) only representative of a very small area around the sampled well. This makes it necessary to have a large number of densely spaced observation wells and bores in order to adequately describe a coastal aquifer.

Complementary to theoretical and field studies, physical models (i.e. small scale representations of the real world in a laboratory environment) were used in order to study the coastal aquifer processes. The most suitable models for saltwater intrusion studies were found to be the Hele-Shaw analogy (Hele-Shaw, 1897, 1899) that uses the similarity between saturated flow in porous media and viscous flow (especially in the vertical direction), along with the ion-motion (which uses the similarity between Darcy's and Ohm's laws in order to visualise movement of an interface with a potential gradient) and the sandbox (a small scale model of a natural porous medium and fluids in a container (e.g. Rumer and Harleman, 1963; Jeanson and Dufort, 1970)) models. Such physical models were often used to confirm analytical solutions and although they have found some application in more recent studies, they are now mostly replaced with direct field measurements.

With advancing time the above mentioned methods were refined and widely applied to study SI and SGD in more detail. Focus was directed on different aspects and some researchers started combining methods in order to gain a better image of the natural processes. The following summary of different studies can be understood as an overview of hydrological research for SI and SGD characterisation carried out in the past 20 years, but is not a complete list thereof. For a more comprehensive review I direct the interested reader to the papers by Oude Essink (2001), Barlow (2003), Ivkovic et al. (2012) and Werner et al. (2013).

One emphasis has been on the influence of tides on SI. Inouchi et al. (1990) analysed the influence of tides on confined aquifers with a freshwater-seawater interface model and a dispersion model, and found the widest mixing zone with maximum saltwater intrusion at mean sea level during the ebb tidal stage. This was confirmed for an unconfined aquifer by Ataie-Ashtiani et al. (1999) who used a variable density model to deduce that tidal activity forces seawater to intrude further inland and thereby also creating a thicker interface. However, the observation was negligible for aquifer depths larger than the tidal amplitudes. A study on the influence 
of tides during semi-diurnal, diurnal and semi-monthly time scales on SGD with automated seepage meters was carried out by Taniguchi (2002) in Japan. From the direct measurements he came to the conclusion that there are changes in SGD with all periods investigated due to the hydraulic gradient created between groundwater and seawater but that the semi-monthly changes (neap-spring tide cycle) have by far the largest amplitude. In Australia Werner and Lockington (2006) conducted groundwater flow dynamics modelling in estuaries and adjacent coastal phreatic aquifers in order to find the long-term influence of the tides on the salinization of the aquifer. They confirmed the main findings of Ataie-Ashtiani et al. (1999) but their long-term simulations, which included several tidal cycles with a range of tidal amplitudes also showed a change in groundwater salinization at the base of the aquifer that was not observed in the single-cycle study by Ataie-Ashtiani et al. (1999). They also found that the tidal induced changes to riparian (transition zone land - river) salinity must be advection driven (i.e. by tidal forces). In a geochemical tracer field experiment Michael et al. (2005) found that seawater intrusion occurs while the saline interface moves landwards. This process was reversed for a seaward moving interface. As these observations were negatively correlated to groundwater recharge, Michael et al. (2005) interpreted a seasonal time lag in the unconfined sedimentary aquifer, which was confirmed through numerical modelling. A field study using observation wells has been carried out by Kim et al. (2006) on a volcanic island. Their focus was on investigating tidally forced changes to the seawater-freshwater interface position. While they found some fluctuations of the saline interface position in a tidal time frame they also concluded that there are only minor changes within a seasonal time frame and that the location of the interface highly depends on the geology that is present. Mulligan et al. (2007) identified paleo-channels as preferential flow paths for groundwater-seawater exchange in their numerical and field studies.

Prior to 2006 a joint project on quantifying SGD with multiple methods (seepage meters, geochemical tracer tests, water balance calculations and numerical modelling) and in different hydrogeologic environments was launched by UNESCO and the International Atomic Energy Agency (IAEA). In their report Burnett et al. (2006) pointed out that regardless of location, tidal variations, both semi-diurnal and during a spring-neap cycle, were the major control force on SGD with larger discharge occurring during low tide. They stressed that multi-method approaches should be used whenever possible and recommended to continue investigations 
on long-term cycles such as seasonal climatic variations. Significant variations in the intertidal salinity structure during a spring-neap tidal cycle were also found by Robinson et al. (2007) through direct field measurements and numerical simulations. In addition they observed higher frequency salinity fluctuations and thereby demonstrated the complexity of the saltwaterfreshwater mixing processes. As already noted by Ataie-Ashtiani et al. (1999), Robinson et al. (2007) also recognised the influence of the beach slope on groundwater flow and salt transport near the shore. Combined field measurements (electrical conductivity logging) and numerical modelling were used by Abarca et al. (2013) to characterise the groundwater dynamics below the SGD. From their results they delineated a growing and shrinking saline circulation cell in the intertidal zone above the SGD with the lunar cycle. In their study they could confirm that recharge into the circulation cell was mainly driven by the high tide elevation and hence more saltwater was intruding during spring tides, whereas SGD was found to be positively correlated to neap tides. Heiss and Michael (2014) conducted a very similar study that also revealed an intertidal circulation cell with a cross-sectional mixing zone that was found to vary with tides, in a spring-neap tide cycle as well as during a seasonal cycle. The seasonal variability turned out to have maximum amplitudes and was determined to be due to oscillations in the water table. Seasonal fluctuations in groundwater-fed wetland salinity correlated to sea level stages were recently determined through field measurements and confirmed by a $2 \mathrm{D}$ variable density model by Wood and Harrington (2015). In essence, they found that salinity peaks in winter were coincident with sea level peaks and that these fluctuations seem to override the influence of seasonal recharge (higher precipitation in winter, hence, lower salinity expected). Heiss et al. (2015) investigated interactions between surface and subsurface processes in the intertidal zone of a sandy beach aquifer with several arrays of moisture sensors and pressure transducers. Again, they confirmed that SGD was primarily controlled by tidal processes and therefore that the surface and subsurface processes in the tidal run up zone are highly coupled. A new interpretation on saline interface control was provided by Levanon et al. (2016) in their numerical study of a unconfined coastal aquifer. The simulation suggested that both the interface and the groundwater table are harmonically influenced by the tides and that the actual control of the movement of the entire freshwater body and hence the saline interface position was mainly related to the attenuation at the groundwater table due to unsaturated flow within the capillary fringe. 
In over 100 years of research for seawater-freshwater exchange processes many studies using theoretical, modelling and field techniques have led to some understanding of the dynamics that occur within the near-shore part of a coastal aquifer. However, there are still knowledge gaps that require further, more intensive, and, most importantly, interdisciplinary research approaches in order to gain a sufficient picture of the processes that drive natural and anthropogenic seawater intrusion. Although the classic hydrologic methods have come a long way and are certainly appropriate tools to investigate the problem, they often lack spatial coverage and therefore rely on statistical interpolation. This may not be a problem in the (utterly rare) case of a homogeneous aquifer, but will lead to errors of unknown dimensions if heterogeneity is present. Almost parallel to the methods and studies discussed above the field of geophysics emerged. Although not directly able to measure hydraulic properties, geophysical methods allow, at least since the 1980's, for a large spatial coverage. Combinations of both hydrological and geophysical methods can overcome the weaknesses of the individual methods and therefore lead to more robust coastal aquifer evaluations. A summary of the history of hydrogeophysics with a special focus on saltwater intrusion detection and characterisation is provided in the following chapter.

\subsection{Hydrogeophysics for saltwater intrusion characterisation}

Geophysical methods have now been applied for hydrologic purposes for decades but hydrogeophysics as an interdisciplinary field has only emerged since the late 1990's (Binley et al., 2015) fueled by vast improvements and new developments of geophysical methods for hydrological purposes. Depending on the research purpose, some geophysical methods are more suitable than others. For example, if the aquifer geology and structure is of interest, seismic and gravitational methods may be suitable, whereas if the question is directed towards the kind of fluid stored in the void space electrical and electromagnetic methods may be a better choice. Only one geophysical technique (nuclear magnetic resonance (NMR)) is currently able to directly detect the volumetric amount of water stored in the subsurface. In aquifers that are geologically well described (for example by available bore core information) SI can be described in terms of interface position and seawater mixing with the direct current (DC) resistivity technique because this method is sensitive to the differing resistance which freshwater and seawater offer 
to electric current.

Geoelectrical methods have been applied to investigate coastal aquifers for almost 80 years, but only since the early 1980's have multi-electrode systems been made available and 2D or 3D surveys possible (Werner et al., 2013). Electrical resistivity tomography (ERT) is used to measure the resistivity distribution along one or several traverses and, in the past decade, has become a standard method in hydrogeophysics (Binley et al., 2015). There are several examples where 2D surface and borehole applications of the DC resistivity method for mapping the saltwater distribution and delineating the saline interface have been successfully carried out on different scales and aquifer systems (e.g. Acworth and Dasey, 2003; Turner and Acworth, 2004; Bauer et al., 2006; Wilson et al., 2006; Comte and Banton, 2007; Hermans et al., 2012). Only very recently have monitoring studies that involve data acquisition over time come to the fore. These range from synthetic modelling studies (Kim et al., 2009) and laboratory scale studies (Wagner et al., 2013) to short-term field investigations (Poulsen et al., 2010; Morrow et al., 2010; Perri et al., 2012; Beaujean et al., 2014) for both surface and borehole applications of the DC resistivity method. De Franco et al. (2009) first developed and installed an extensive ERT experiment for time-lapse monitoring of saline intrusion in the coastal aquifer near Venice (Italy). Within a time period of nine months, they found a seasonal resistivity change in the shallow phreatic sand aquifer. Saline intrusion was observed during autumn and winter months and the opposite behaviour for the spring and summer time. They concluded that a time lag existed between pumping activity and saline intrusion, and hence, a negative relationship would characterise the behaviour of the coastal aquifer at this location. Based on their idea, the automated time-lapse electrical resistivity tomography (ALERT) system (Ogilvy et al., 2009) was installed in Almeria (Spain), where SI is already regarded as being a problem. The completely automated monitoring system measured resistivity data over a period of three years, and Nguyen et al. (2009) interpreted the shape of the salt- water wedge intruding the aquifer as being described by the classical Ghyben-Herzberg relationship (Badon Ghyben, 1889; Herzberg, 1901). They also correlated water electrical conductivity measurements from two boreholes along the profile with in-hole resistivity measurements and inferred that the ERT images must be mainly influenced by the amount of total dissolved solids rather than the lithology. Furthermore, Ogilvy et al. (2009) found a relation between groundwater pumping activity and saline intrusion, but could rule out saltwater movement due to natural features at 
this site.

It is evident that only a few field studies have been carried out using geophysical methods to image the processes taking place in a coastal aquifer over a long-time period. The value of geoelectrical methods for this purpose has been widely demonstrated and has proven itself to be a powerful and economic tool for investigations on a larger spatial scale than would be possible with classical hydrological field approaches such as pumping tests, core analysis, or well monitoring. However, to increase the understanding of the highly complex processes taking place in sea bound aquifers, it is necessary to undertake extensive long-term studies both in geologically similar and in different coastal aquifer environments. Furthermore, information from hydrologic investigations should be taken into consideration for geophysical modelling and vice versa, subsurface characteristics found from geophysical field studies should be used to lead to more stable hydrological simulations, thus, improving the methods used by both fields.

\subsection{Hypothesis and field programme}

In light of the above outlined studies and their results, seawater intrusion certainly depends on the location of investigation. However, to an extent, this can be generalised for hydrogeologically similar areas. With respect to climate change time scales, for studies that only cover monitoring periods of roughly one year, changes in mean seawater level can be considered negligible. Hence, the amount of seawater intruding a coastal aquifer should be depending on how much freshwater is available at any time.

Main hypothesis:"Freshwater availability/recharge is the major driving force for seawater intrusion into a shallow sandy coastal aquifer."

Freshwater availability and therefore the amount of freshwater opposing seawater intrusion depends on several factors, such as the amount of recharge (i.e. through rainfall), the amount of evaporation and evapotranspiration, and the amount of freshwater that is removed from the aquifer for example by pumping activities. With this in mind, it is hypothesised that 
there would be an observation of greater seawater intrusion into the shallow coastal aquifer during summer months when there is less precipitation and hence natural recharge of the aquifer, as well as intensified evaporation/evapotranspiration and more abstraction of fresh groundwater through wells and bores for irrigation and other purposes. This is illustrated the highly simplified conceptual model in Fig. 1.2(a). In winter months, with increased rainfall, decreased evaporation and decreased water pumping, this process would then be expected to reverse and less saline mixing should be observed in the shallow aquifer (Fig. 1.2(b)). With respect to electrical conductivity, this should then be observed as an increase during summer when more seawater entering the aquifer would lead to an increase in total dissolved solids. The opposite would then be expected to be observed in winter, where a larger groundwater flow would lead to a lowered amount of total dissolved solids and hence, decreased electrical conductivity.
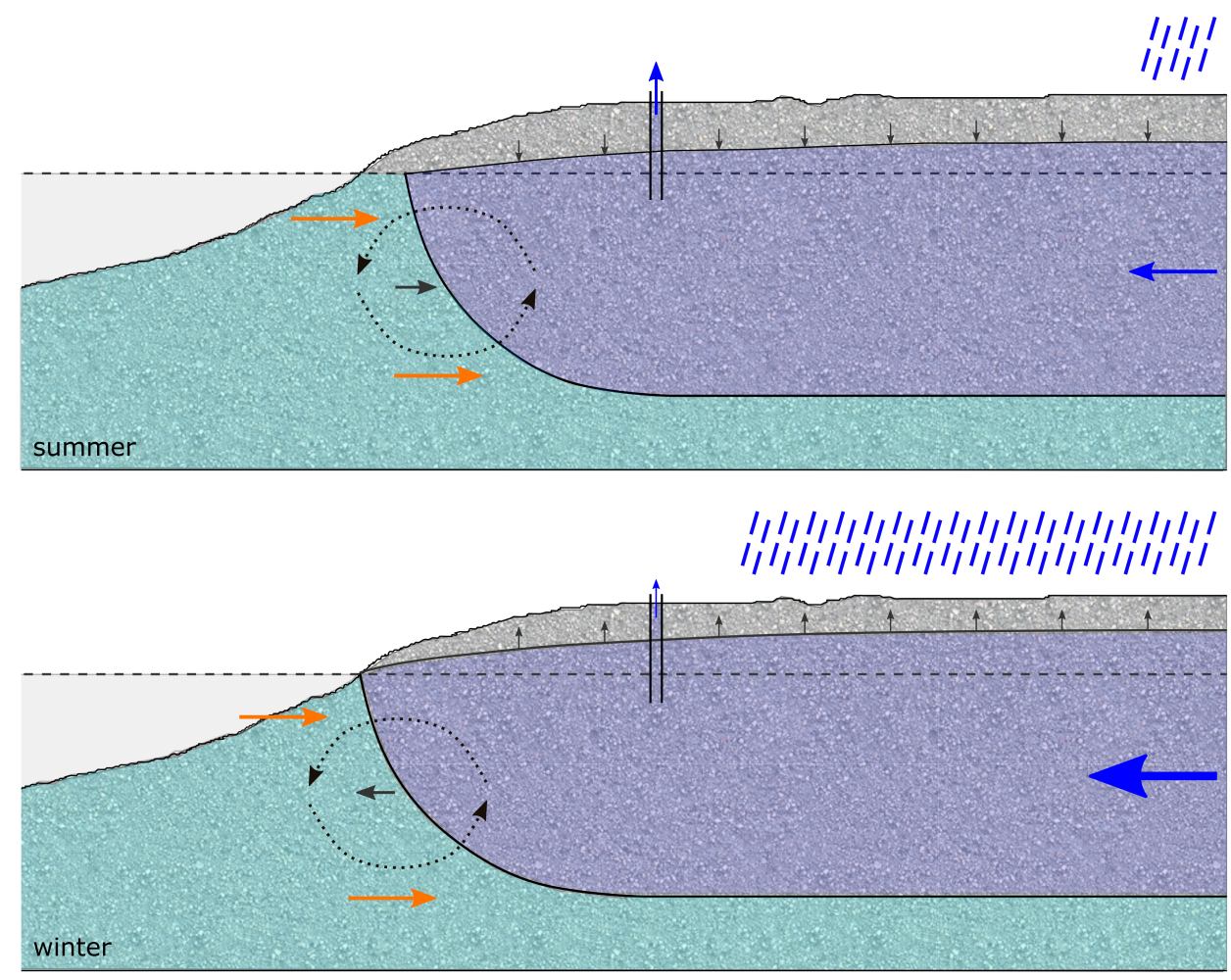

FIGURE 1.2: Conceptual model of main hypothesis.

A long term electrical conductivity (EC) record is available from a monitoring bore (R26/6587, GWRC groundwater monitoring data base) some $3 \mathrm{~km}$ away from the coast line and at a depth of $10.5 \mathrm{~m}$ from the surface at the field location (see Chapter 2 for a detailed overview of the area). EC was measured approximately every three months for more than 20 years. In Fig. 1.3 
EC data from the years 2000 to 2015 are imaged and roughly segmented into summer and winter half-years. Following the main hypothesis, it would be expected to see EC peaks in the orange segments and EC troughs in the blue segments. From Fig. 1.3 it becomes apparent that this is not strictly the case, however, and the trends seemingly change randomly over time. Whether this is a an indication for the EC signal not having a seasonal component, or if this pattern may be tied to complications related to EC measurements in the field cannot be easily answered. This project therefore aims at studying groundwater dynamics with several methods in order to provide a more robust assessment of how a coastal aquifer responds to environmental changes in different seasons.

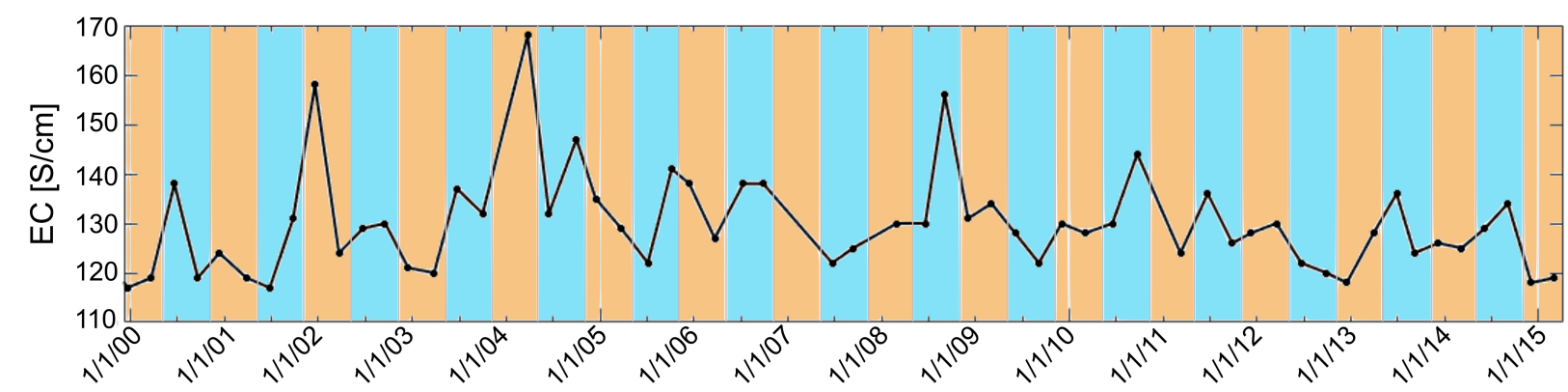

FIGURE 1.3: Long term (15 years) EC sampling in well R26/6587 (GWRC monitoring data base) about $3 \mathrm{~km}$ away from the coast line. Orange segments mark warmer months (summer), whereas the blue segments highlight those where temperatures are lower (winter).

The aim of this thesis is to use the DC resistivity method for investigating how much seawater mixing does occur at what times within a shallow coastal aquifer during the course of a year. Along with the main hypothesis further questions need to be asked in addition. These are:

$\times$ Is the DC resistivity method suitable to monitor salinity changes in a coastal aquifer?

$\times$ How does urbanisation influence saline mixing in an unconfined costal aquifer?

$\times$ What is the influence of tidal forcing on the DC resistivity monitoring for seasonal salinity changes?

In order to test the hypothesis and answer associated questions, an extensive field programme was designed, which is outlined below.

1. Identify a suitable field site and establish the optimal DC resistivity method settings to address monitoring of seasonal salinity changes within the aquifer. 
2. Use these settings to consistently monitor a number of transects within the field area over the course of a year.

3. Monitor the impact of tides on DC resistivity signals by repeating geoelectrical measurements along one transect with a one-hourly frequecy during a diurnal tidal cycle.

4. Make use of the extensive environmental monitoring system of the Greater Wellington Regional Council (GWRC) in order to obtain information about rainfall intensity and groundwater levels in the coastal aquifer.

5. Use the obtained field information and 3D forward modelling in order to determine the best possible positions and dimensions for a cross-borehole study of the mixing zone.

6. Use three 40-m deep boreholes in order to enable cross-borehole and surface-to-borehole DC resistivity measurements at one of the surface transect locations and monitor higher resolved salinity changes in the mixing zone over the period of one year.

Unfortunately, field permissions for drilling the deep monitoring boreholes could not be obtained within the first half of this project. This was due to several reasons but came ultimately down to the local council putting an unfeasible financial constraint on the permission process. Therefore, the initial project outline needed to be re-structured about half-way through. In order to achieve a better resolved image with respect to the time domain and to investigate if the pilot study was reproducible elsewhere, resistivity measurements were repeated at a higher frequency and the field area was shifted to a location north of the initial study area (i.e. similar hydrogeological environment, different surface conditions and access to shallow groundwater bores). The shift to a new field site was also a strategic step as it was aimed to substitute the permit-refused DC resistivity borehole measurements with hydrochemical monitoring through the vastly available network of shallow bores at the new field site in order to get an independent characterisation of seawater mixing in the unconfined aquifer over a seasonal cycle. At this new field location, not only many shallow bores were available, but there have also been previous studies carried out with a focus on seawater intrusion characterisation. In addition to the original questions raised, the following questions arose to be answered.

$\times$ Is the observed seasonal saline mixing behaviour at the original unconfined coastal aquifer consistent with observations from a hydrogeologically similar coastal aquifer? 
$\times$ How does seasonal hydrochemical groundwater information support the DC resistivity monitoring?

$\times$ What are the long-term implications at a specific field site that has a record of previous hydrochemical and geoelectrical measurements?

$\times$ Is it possible to extract additional aquifer characteristics from extensive DC resistivity monitoring series?

$\times$ Are there any changes to the resistivity signal during a tidal monitoring depending on the season this is measured in?

Keeping the same focus on the main hypothesis, the field programme needed to be adjusted in order to incorporate the new questions. The below list reflects changes to the original field programme and shows the adjustments needed to be carried out in order to answer the additionally asked questions.

1. Identify a suitable field site and establish the optimal DC resistivity method settings to address monitoring of seasonal salinity changes within the aquifer.

2. Use these settings to consistently monitor a number of transects within the field area over the course of a year.

3. Monitor the impact of tides on DC resistivity signals by repeating geoelectrical measurements along one transect with a one-hourly frequecy during a diurnal tidal cycle.

4. Make use of the extensive environmental monitoring system of the Greater Wellington Regional Council (GWRC) in order to obtain information about rainfall intensity and groundwater levels in the coastal aquifer.

5. Use the obtained field information and 3D forward modelling in order to determine the best possible positions and dimensions for a cross-borehole study of the mixing zone.

6. Use three 40-m deep boreholes in order to enable cross-borehole and surface-to-borehole DC resistivity measurements at one of the surface transect locations and monitor higher resolved salinity changes in the mixing zone over the period of one year. 
7. Repeat the surface DC resistivity monitoring with double frequency at the new field location (i.e. every 30 days) over the course of a year.

8. Obtain permission to use a number of shallow bores tapping into the unconfined aquifer and sample these in winter and summer for the rest-duration of the project.

9. Measure a resistivity transect covering the entire width of the new study area at the same times as the hydrological samples are taken.

10. Repeat the tidal monitoring at the new site over part of a diurnal tidal cycle in order to find seasonal changes of tidal influences.

This led to a data set of more than 150 individually measured DC resistivity transects. 45 groundwater samples were analysed in three sessions and a well logger installed in one of the shallow bores measured continuously temperature, electrical conductivity and groundwater level over a period of 8 months, providing roughly 23'000 data points of each of these parameters. Although, not all of the data obtained could also be used for the thesis, there was sufficient quantity to either fully answer the above questions or at least give a good indication of the processes involved.

\subsection{Objectives and structure of this thesis}

The main goals of this $\mathrm{PhD}$ thesis are to investigate the potential of the application of the $\mathrm{DC}$ resistivity technique for seawater intrusion characterisation in a shallow coastal aquifer on different time scales and to combine the knowledge gained from the long-term monitoring study with directly measured hydrological data in order to provide an extensive long-term field characterisation of a shallow coastal aquifer. In the long run, this study will contribute towards filling knowledge gaps related to seawater intrusion in general by investigating which processes play a leading role at the site of investigation and therefore provide a tool for future management of groundwater in the study area and areas of similar hydrogeological character. The thesis is built on observations from field data collected during the term of the PhD project. Most applications used to collect and process the data are not new but some of them are applied to the problem of seawater intrusion monitoring for the first time. The geoelectrical traversing 
method was chosen as the most suitable geophysical method due to its sensitivity to fluid resistivity contrasts. Test measurements with other methods such as refraction seismic and ground penetrating radar were not producing any useable results, which is possibly due to the loose sedimentary nature of the field sites.

All publications resulting from this thesis are based on the thesis itself for which I am the sole author. My co-author and supervisor Malcolm Ingham had an advisory role for the paper published from Chapter 4 prior to thesis submission. The two papers originating from Chapters 5 and 6 were submitted after defending the thesis. References for the papers can be found on the respective title pages.

The field sites used to investigate seawater intrusion behaviour are introduced in Chapter 2 . There, I first give an overview of the hydrogeology of the wider region before going into more detail for each of the two groundwater zones. In a separate section I then discuss cross-sections from previous studies in the area and bore core information obtained near the two field sites. This builds the base for understanding the geoelectrical models and helps understanding processes relevant for seawater intrusion at these locations.

In Chapter 3 I give an extensive overview of the techniques used to collect and process the field data. This is split into three parts starting with the theory for the geophysical aspects of this project, which is followed by a discussion of the hydrological applications used. The third part of this chapter is dedicated to tools used for time series analysis of the geoelectrical monitoring data sets.

Chapter 4 describes the results and implications from the long-term DC resistivity monitoring of seawater intrusion at two locations on the Kapiti Coast collected during two subsequent years in terms of a seasonal cycle. I attempt to determine if a natural seasonal cycle of seawater intrusion is apparent at the study sites and in what spatial and temporal dimensions seawater mixing in the shallow coastal aquifer changes within a year. The part covering the original field location (Waikanae) of this chapter has been published in Near Surface Geophysics.

Chapter 5 has its focus on seawater intrusion monitoring on a smaller time scale. It investigates the influence of tidal wave action on seawater mixing in a coastal aquifer. This is discussed in 
terms of resistivity changes across a tidal cycle but I also investigate the possibility of using statistical time series analysis tools in order to characterise the hydrological properties of the subsurface. The chapter shows the potential of a simple method to determine preferential flow paths for seawater intrusion. Seasonal impacts on tidal resistivity monitoring studies can be found in Appendix C. A paper presenting methods and analysis of Chapter 5 is currently under revision for publication in Water Resources Research.

In Chapter 6 I investigate if and how the chemical groundwater composition changes in a yearly cycle. The data set was collected at the field site of Te Horo and provided the reason to shift the location focus there during the second half of the $\mathrm{PhD}$ project. An additional aspect of this chapter is the discussion of well logging data collected at the same site over the course of about eight months. The data and combined discussion of this part of the chapter was submitted as a paper to the Journal of Hydrology. In addition to the seasonal monitoring, I used data from two previous studies at the same field location in order to investigate seawater intrusion potential over a longer time span of 13 years.

Finally, in Chapter 7 I make an attempt at combining all the data previously discussed. This leads to a comprehensive interpretation of seawater intrusion into a shallow coastal aquifer and can be understood as the summary of the interdisciplinary large scale and long-term field investigation. I there outline the potential of seawater intrusion for the shallow groundwater zone of the study site and characterise the processes involved. It embeds the conclusions drawn from the PhD project and in the end I also provide an outlook of possible areas for future research.

The Appendices contain any figures and tables that were not regarded necessary to present in the text, but the reader may have an interest to see. These are referred to in the text. Additional resistivity data collected in the Te Horo groundwater zone to investigate the interface shape close to the model boundary and saline mixing velocity estimates from the geoelectrical models are also discussed here, along with modelling studies. All of these are referred to in the main text.

A glossary of abbreviations can be found on page xix. 



\section{CHAPTER 2}

\section{The field area}

"The way to do fieldwork is never to come up for air until it is all over."

Margaret Mead, cultural anthropologist (1901 - 1978)

The focus of this chapter is on the field area. I first discuss the regional hydrogeology, then give a more detailed discussion of the groundwater zones associated with the two specific field locations by going into more detail about their hydrological characterisation and presenting the coastal geology through cross-sections and bore core information. 


\subsection{Scope}

New Zealand is a country surrounded by seawater. From a recent estimate 711 billion cubic meters of groundwater (Moreau and Bekele, 2015) are stored in New Zealand's aquifers. They provide almost half of all the water currently used (GNS Science NZ, 2016), with the unconfined aquifers accounting for approximately $96 \%$ of this groundwater (Moreau and Bekele, 2015). With increasing demand of freshwater for domestic, agricultural and industrial supply many of these aquifers will (or already are) experiencing an increased stress. If recharge cannot keep up with abstraction rates the groundwater pressure will decrease and aquifers located at the coast will be subject to seawater intrusion. This problem has been recognised at regional and governmental level in New Zealand (e.g. Aitchinson-Earl et al., 2003; Callander et al., 2011; Thomas and Callander, 2013; Gyopari et al., 2014; Parliamentary Commissioner for the Environment, 2015; Weir and Davidson, 2016). In some places seawater monitoring wells (so-called Sentinel wells) were installed close to the coast within the last decade in order to generate an early seawater intrusion warning network.

The field site that was monitored during this $\mathrm{PhD}$ thesis was initially suggested by the Greater Wellington Regional Council (GWRC) because they regarded seawater intrusion to be an evident issue for the coastal aquifers along the Kapiti Coast (map see Fig. 2.1). Since 2005 Waikanae, a town on the Kapiti Coast, has installed a network of deep bores that top up the freshwater supply, normally taken from the Waikanae River, in dry summer months. However, since 2013 this network has been expanded in order to be able to extract large volumes of groundwater for river water top up. From the 2014 annual Waikanae Borefield report (Fraser et al., 2014) it appears that one production bore in close vicinity to the coast $(<1 \mathrm{~km})$ had to be decommissioned due to the potential threat of seawater intrusion. Additional SI monitoring wells have been installed recently and several groundwater models were produced in order to estimate the magnitude of a possible SI threat to Kapiti's coastal aquifers (e.g. Jones and Gyopari, 2005; Gyopari et al., 2014). However, it is still unclear what the leading processes of SI in the area are because no long-term field monitoring information is available which directly investigates different parameters within different time scales. In the following sections subsurface information of the field area from previous studies and bore log information are discussed in order to provide a basis for geoelectrical model and hydrochemical result interpretations. 


\subsection{Hydrogeology of the Kapiti Coast}

The Kapiti Coast is a narrow coastal plain situated west of the Tararua Mountain Range between Paekakariki and Otaki on the west coast of the lower North Island of New Zealand (Fig. 2.1), and comprises a mixture of alluvial and marine sediments underlain by greywacke basement rocks (Tidswell, 2009). Active uplift of the Tararua Range by structural deformation due to the convergent tectonic setting of New Zealand has led to large sedimentary basins, such as the South Wanganui Basin, of which the Kapiti Coast is located at the south-western margin (Gyopari et al., 2014). These deformations result in a horst and graben structure of the basement rocks underlying the Kapiti Coast, which is likely to have a significant influence on the subsurface hydrology (Ewig, 2009). The last marine transgression, approximately 6500 years BP (Tidswell, 2009), built up the uppermost sequence of the sedimentary deposits which comprises the shallow coastal aquifer of the area. It consists of sand dunes, interdunal swamps and beach sands that are still forming the present landscape. Rivers that drain the Tararua Range westwards cut into the sediment deposits. Jones and Baker (2005) identified three main groups of deposits building up the Kapiti Coast aquifers. Depending on the environment they were deposited in, they are termed 1) glacial and interglacial deposits, 2) post-glacial beach and dune sands and 3) recent river gravels. While the first group of glacial and interglacial sediments describe a thick, poorly stratified, confined aquifer system along the entire coast at depths usually below 30 - 40 meters, the near-surface post-glacial sands, overlying the glacial and interglacial sediments, thin out towards the Tararua Range and host a mostly unconfined aquifer which becomes semi-confined at larger depths closer to the coast. Transmissivity is moderate for the glacial and interglacial unit with yields of $500-1000 \mathrm{~m}^{2} /$ day (Jones and Baker, 2005) and recharge is mainly due to rainfall. The shallow aquifer built up by the post-glacial sands and dunes has only a low transmissivity $\left(<100 \mathrm{~m}^{2} /\right.$ day) and is considered to be only suitable for domestic groundwater use. The groundwater flow in the coastal aquifers is towards the coast and the deeper confined units are thought to have their natural discharge point somewhere offshore (Tidswell, 2009).

Drill logs conducted during different studies in the area suggest a classification of six hydrostratigraphic units (Table 2.1) within the first 120 meters below the surface (Utting, 2013; Gyopari et al., 2014), the latest of which were deposited during the Holocene in a climate similar to the one today. Some of the units do not occur in all wells drilled in the region, indicating that some 


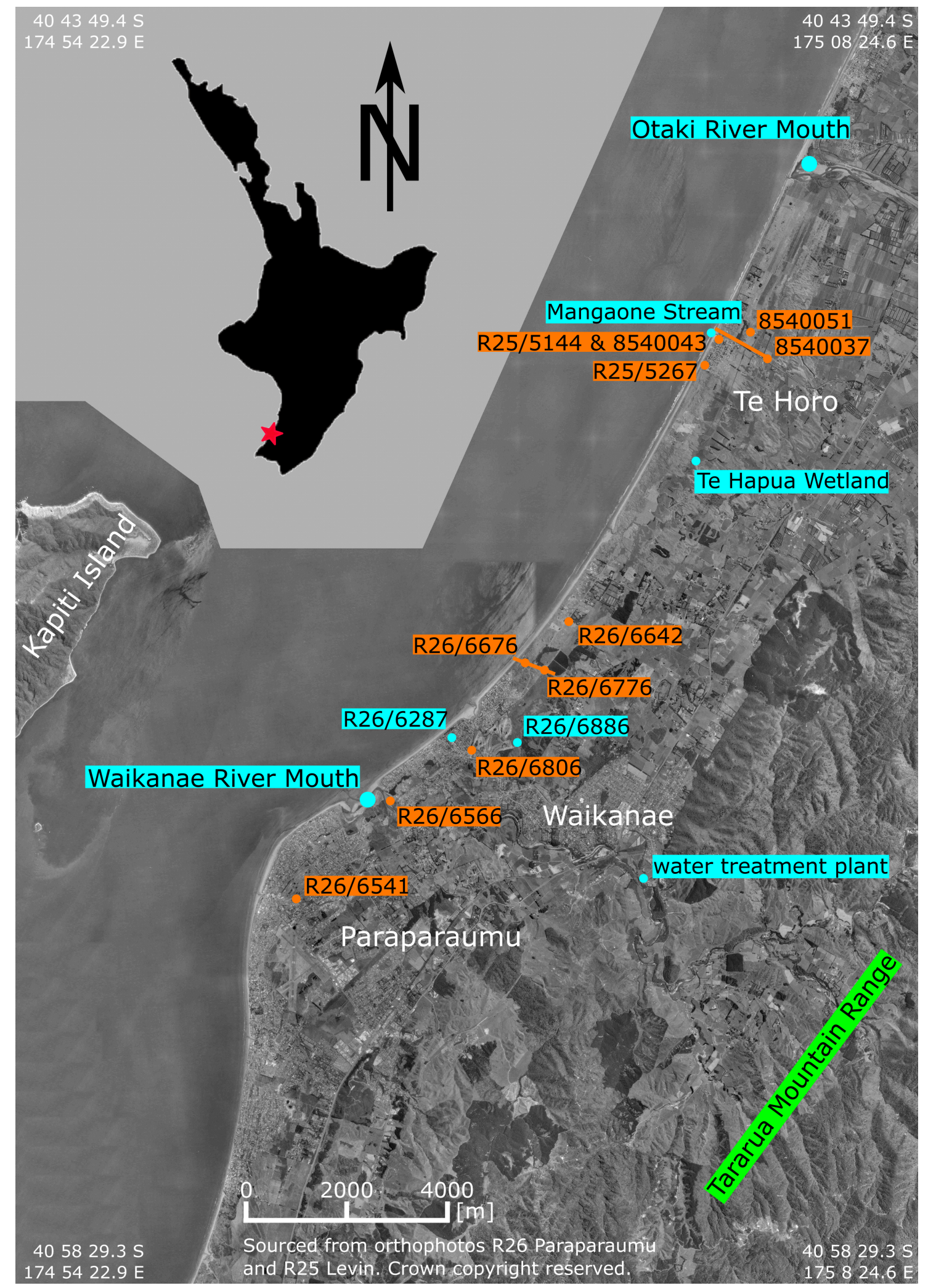

FIGURE 2.1: Map of the field area (Kapiti Coast) in New Zealand showing the Waikanae/Paraparaumu (referred to as Waikanae field area due to being located in the Waikanae groundwater zone) and Te Horo field areas. The Tararua Mountain Range where most of the groundwater recharge happens, runs SW-NE in the eastern part of the study area. The regional council measures groundwater stage (amongst others) in wells R26/6287 and R26/6886, and at the Te Hapua wetland. Rainfall data is obtained from the water treatment plant and the Te Hapua wetland. Tidal stage information comes from NIWA for the Waikanae- and Otaki River mouths. 
of the aquifers are connected in parts, but might be limited by a hydraulically less conductive unit at other locations. The spatially large distances between the wells and the relatively large heterogeneity of sedimentation in the Waikanae area also make a connection of the stratigraphic information for regional subsurface interpretation a challenging task.

TABLE 2.1: Hydrostratigraphic units and their description for the aquifers found along the Kapiti Coast (modified from Gyopari et al. (2014)). Not all units are present everywhere along the coast, as indicated for example in Fig. 2.2.

\begin{tabular}{|c|c|c|}
\hline $\begin{array}{l}\text { Hydrostratigraphic } \\
\text { Unit }\end{array}$ & Description & Spatial/Depth Distribution \\
\hline Q1 - Holocene sand & $\begin{array}{l}\text { medium to fine sand with lenses of } \\
\text { gravel, silt and peat, hosting a shallow } \\
\text { unconfined aquifer system primarily } \\
\text { recharged by local rainfall and show- } \\
\text { ing a relatively low bulk conductivity } \\
(K \sim 2-5 \mathrm{~m} \text { /day) }\end{array}$ & $\begin{array}{l}\text { extends from west of Waikanae to the } \\
\text { coast forming a wedge shaped deposit } \\
\text { with increasing thickness from around } \\
20 \mathrm{~m} \text { inland up to } 40 \mathrm{~m} \text { near the coast }\end{array}$ \\
\hline Q1 - gravel & $\begin{array}{l}\text { coarse, heterogeneous deposits of } \\
\text { sand and gravel that host a highly } \\
\text { permeable unconfined aquifer with } \\
\text { high hydraulic conductivity of } K \sim 50 \text { - } \\
100 \mathrm{~m} / \text { day and are hydraulically con- } \\
\text { nected to surface water }\end{array}$ & $\begin{array}{l}\text { underlies the recent floodplain of the } \\
\text { Waikanae River and the former chan- } \\
\text { nel of the Waimeha River and forms } \\
\text { a relatively narrow, elongate deposit } \\
\text { generally between } 10-15 \text { m thick }\end{array}$ \\
\hline Q2 - gravel & $\begin{array}{l}\text { moderately to poorly sorted alluvial } \\
\text { gravel with varying amounts of silt and } \\
\text { sand, forming a low to moderate con- } \\
\text { ductivity unconfined to semi-confined } \\
\text { aquifer ( } K \sim 10-50 \mathrm{~m} / \text { day) and hy- } \\
\text { draulically connected to the overlying } \\
\text { Q1 sand aquifers }\end{array}$ & $\begin{array}{l}\text { extends towards the coast (and further } \\
\text { offshore) under the Q1 Holocene sand }\end{array}$ \\
\hline $\begin{array}{l}\text { Q3 - gravel, sand } \\
\text { and silt }\end{array}$ & $\begin{array}{l}\text { heterogeneous deposits of sand, silt } \\
\text { and gravel with a permeability suffi- } \\
\text { ciently low to restrict hydraulic con- } \\
\text { nection between the Q2 and Q4 sedi- } \\
\text { ments }\end{array}$ & $\begin{array}{l}\text { typically about } 10-15 \mathrm{~m} \text { thick and may } \\
\text { become coarser inland and indistin- } \\
\text { guishable from Q2/Q4 deposits }\end{array}$ \\
\hline Q4 - gravel & $\begin{array}{l}\text { gravel and sand similar to Q2 sedi- } \\
\text { ments, forming a moderately perme- } \\
\text { able semi-confined aquifer }\end{array}$ & $\begin{array}{l}\text { thickness varies from less than } 10 \mathrm{~m} \\
\text { to about } 20 \mathrm{~m}\end{array}$ \\
\hline
\end{tabular}


Table 2.1 - continued from previous page

\begin{tabular}{|c|c|c|}
\hline $\begin{array}{l}\text { Hydrostratigraphic } \\
\text { Unit }\end{array}$ & Description & Spatial/Depth Distribution \\
\hline Q5 - sand and silt & $\begin{array}{l}\text { medium to fine sand with varying hy- } \\
\text { draulic conductivity of } K \sim 1-20 \mathrm{~m} /- \\
\text { day due to varying amounts of silt } \\
\text { and organic material; it's forming an } \\
\text { aquitard separating the water-bearing } \\
\text { intervals in the Q2/Q4 and Q6 gravel } \\
\text { deposits in most areas, but may also } \\
\text { contain higher yielding relatively well } \\
\text { graded medium to fine sand layers }\end{array}$ & $\begin{array}{l}\text { occurs as a continuous layer to the } \\
\text { west of the basement high (Fig. 2.2) } \\
\text { and may be discontinuous in the area } \\
\text { overlying the basement high (or has a } \\
\text { significantly different character with } \\
\text { higher hydraulic conductivity) }\end{array}$ \\
\hline Q6 - gravel and sand & $\begin{array}{l}\text { poorly sorted, weathered gravel in } \\
\text { silty, sandy matrix hosting a low } \\
\text { to moderate yielding semi-confined } \\
\text { aquifer system with low to moder- } \\
\text { ate conductivity ( } K \sim 10-30 \mathrm{~m} / \text { day), } \\
\text { which may decrease inland due to in- } \\
\text { creasing proportion of silt }\end{array}$ & $\begin{array}{l}\text { strata inferred to dip westwards fol- } \\
\text { lowing the profile of the Waikanae fan } \\
\left(\sim 1^{\circ}\right)\end{array}$ \\
\hline $\begin{array}{l}\text { Greywacke } \\
\text { basement }\end{array}$ & $\begin{array}{l}\text { well indurated siltstone and mudstone } \\
\text { that may contain secondary permeabil- } \\
\text { ity in joints and fractures but is essen- } \\
\text { tially considered to form the ground- } \\
\text { water basement }\end{array}$ & $\begin{array}{l}\text { extends from the Tararua foothills to } \\
\text { the east of the Waikanae groundwater } \\
\text { zone with a large bedrock high (lift- } \\
\text { ing basement to about } 30-50 \text { m below } \\
\text { ground level (Ewig, 2009) compared } \\
\text { to the east where it is at about } 90 \mathrm{~m} \\
\text { below ground level) underlying the } \\
\text { Waikanae township associated with } \\
\text { normal NE-SW trending faults along } \\
\text { the coast, but going to larger depths } \\
\text { towards the coast }\end{array}$ \\
\hline
\end{tabular}

\subsection{Waikanae groundwater zone}

\subsubsection{Aquifer hydrology}

A schematic illustration of the six hydrostratigraphic units in the Waikanae area is given in Fig. 2.2. Groundwater is generally found throughout the entire stratigraphic sequence (Q1 Q6) with differences occurring mainly in the relative permeability defining the units as either aquifers or aquitards. The Waikanae groundwater zone has around 4500 bores (most of them 
$<6 \mathrm{~m}$ in depth) and is the most developed groundwater zone in the area and, due to many research (e.g. Welch, 2004; Ewig, 2009; Allen, 2010; Morrow et al., 2010) and industrial projects carried out at this locality, also by far the best characterised one (Gyopari et al., 2014). An extensive unconfined aquifer made up from the Holocene sand and gravel deposits (Q1) is found at the surface extending to depths of approximately $30-40 \mathrm{~m}$. It is recharged by local rainfall and flow loss from the Waikanae River. Welch (2004) found that the time-lag and magnitude between rainfall, river stage and groundwater stage levels increases for bores with an increasing distance from the Waikanae River and concluded that outside the Q1 alluvial gravels the recharge must be mainly due to rainfall rather than changes in the river stage. Gauging observations along the Waikanae River suggest a recharge of the aquifer between the water treatment plant (Fig. 2.1) and a park located further downstream (loss of about $300 \mathrm{l} / \mathrm{s}$ ). Closer to the coast the river experiences a discharge from the aquifer to its baseflow (Gyopari et al., 2014). This indicates downward vertical groundwater flow upstream and upward vertical groundwater flow downstream near the coast. Similar behaviour was also observed for the smaller Waimeha Stream, a side stream of the Waikanae River (Osborne, 2006).

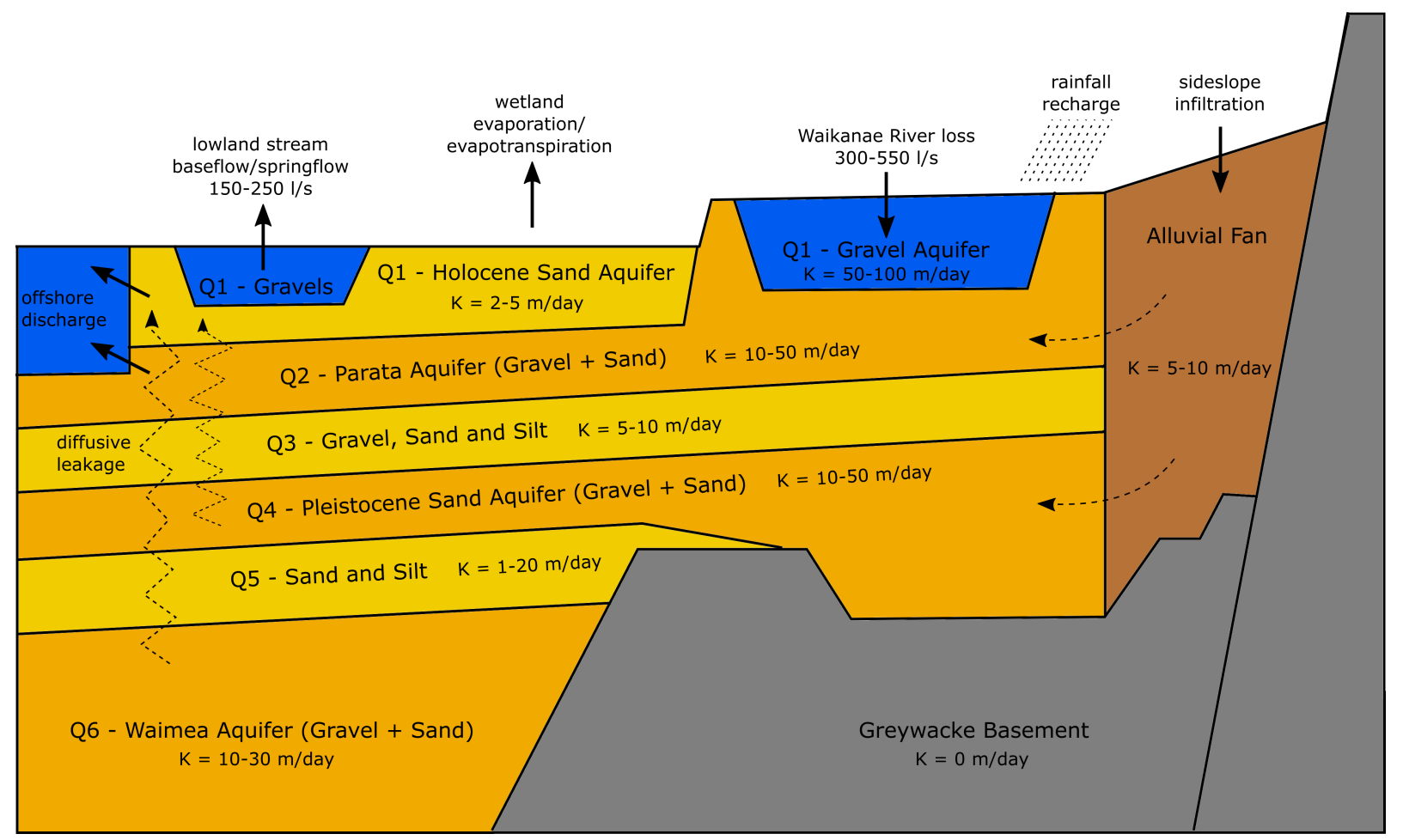

FIGURE 2.2: Schematic illustration of the Waikanae groundwater zone with the six hydrostratigraphic units (Q1-Q6), their estimated hydraulic conductivity and indicated preferential flow paths as suggested by Utting (2013) and Gyopari et al. (2014). The figure has been modified from Gyopari et al. (2014). 
In general, the water table relative to the surface elevation is found at shallower depths closer to the coast. The discharge point of the shallow aquifer is likely at the coastal margin and the aquifer is also connected to wetlands and other small streams in the area (Gyopari et al., 2014). Some groundwater monitoring wells close to the coast indicate that the shallow aquifer is influenced by daily and monthly tidal cycles near the shoreline. Groundwater table height measurements from the Greater Wellington Regional Council (GWRC) monitoring network around Waikanae also show a seasonal variation, typically showing the lowest levels in April and the highest stands in October (Fig. 2.3a). This agrees with precipitation records at the Waikanae water treatment plant (Fig. 2.3b) and indicates a strong correlation between recharge and rainfall intensity along with the rapid recharge from surface water of the shallow coastal aquifer mentioned by Gyopari et al. (2014) and Utting (2013). Underneath the Holocene sand and gravel deposits late Quaternary alluvial material (Q2 - Q6) host an extensive groundwater source in a heterogeneous stratified system that comprises multiple leaky water-bearing intervals, which become more confined with increasing depth as found from past pumping tests carried out in the area (Gyopari et al., 2014). From piezometric head measurements in the coastal aquifer it is found that the vertical hydraulic gradient is upwards for the deeper aquifers, meaning that they are not subject to contamination from the shallower overlying units (Tidswell, 2009). However, this is somewhat contradictory to the theory that groundwater recharge in the lower glacial and interglacial units is mainly due to rainfall as stated by Jones and Baker (2005) and reflects the large heterogeneity of the alluvial deposits and indicates that the responses to recharge and discharge are subject to large variations in both spatial and vertical direction (Mzila, 2013).

In 2005 the Waikanae Borefield was installed in order to supply sufficient freshwater resources for public consumption during dry summer months when river water take is restricted. These wells penetrate to depths of around 40 - $100 \mathrm{~m}$ below surface, sourcing groundwater from the deeper Parata, Pleistocene Sand and Waimea aquifers (Q2, Q4 and Q6 units) (URS, 2010). Utting (2013) estimated a maximum drawdown of up to $0.5 \mathrm{~m}$ in the Holocene sand (Q1) and up to $15 \mathrm{~m}$ in the deeper (abstraction) aquifers within the next 28 years due to pumping activities from the Waikanae Borefield. This could potentially lead to saline intrusion, especially into the deeper aquifer units, due to a lowered freshwater pressure. 

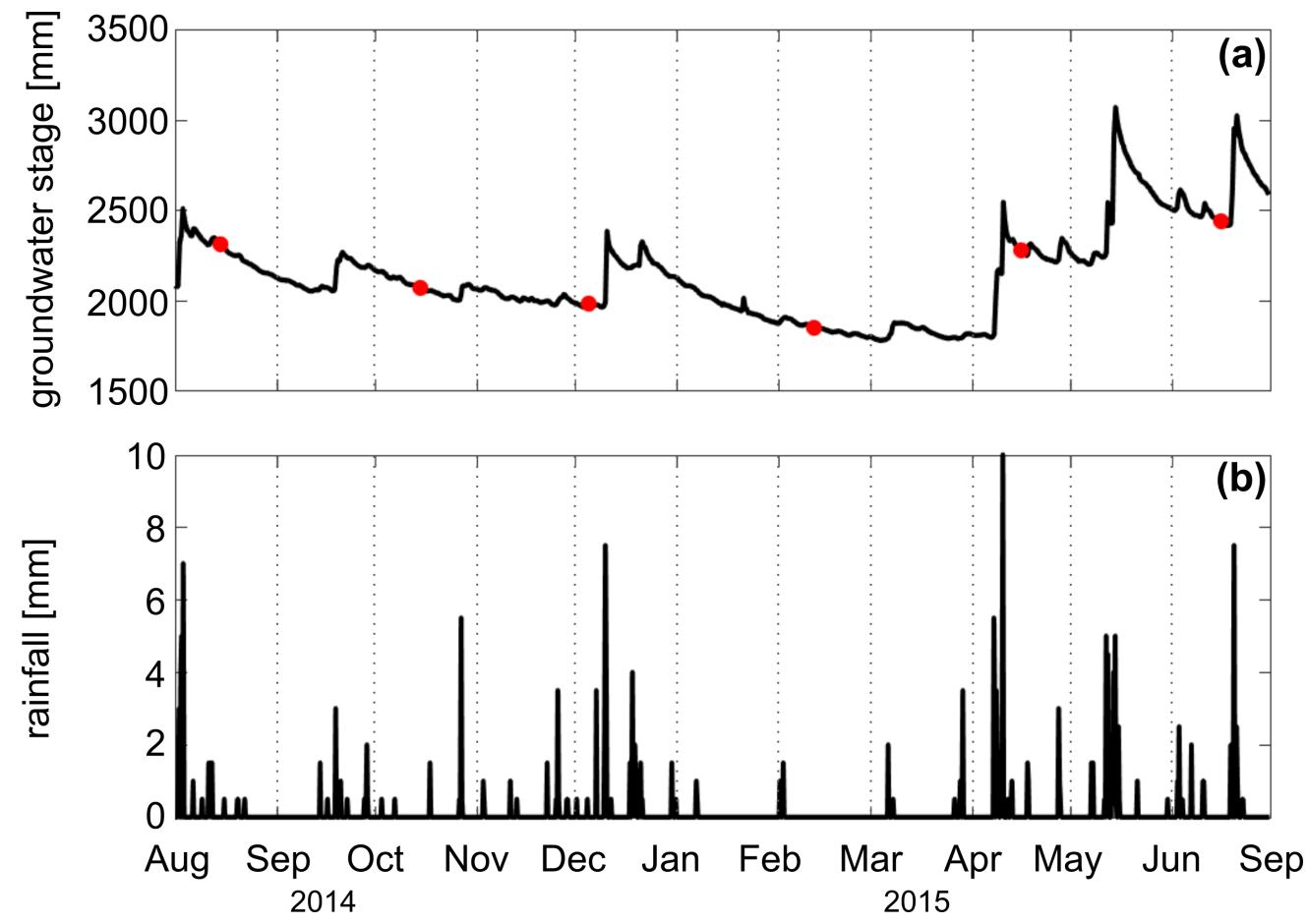

FIGURE 2.3: Correlation between groundwater table height near the coast and precipitation records from the Tararua foot range. a) Groundwater table level data for the Waikanae DC resistivity monitoring period measured in well R26/6287 (GWRC monitoring network). Dots mark the acquisition dates of resistivity data on the N2 profile (see Fig. 4.1). b) Hourly rainfall data for the DC resistivity monitoring period as recorded at the Waikanae Water Treatment Plant (locations see Fig. 2.1).

\subsubsection{Coastal geology}

The coastal geology for the Waikanae groundwater zone for the uppermost 30 - $40 \mathrm{~m}$ (Q1 Holocene deposits) is reflected in more detail from the bore cores shown in Fig. 2.4 and the transect constructed from three additional bore cores as illustrated in Fig. 2.5. The location of these bores in respect to the field area can be found in Fig. 2.1. All bores are located within less than $700 \mathrm{~m}$ of the coast. To the south of the Waikanae study area two bores (R26/6541 R26/6566 - Fig. 2.4) depict the shallow unconfined aquifer, made up of a mixture of sand and sand with varying amounts of silt, clay, gravel and wood, to reach depths of about $40-25 \mathrm{~m}$ below sea level. Below these levels a more gravelly unit is found likely reflecting the lower lying Q2 aquifer unit. While these two bores do not suggest any major gravel contents in the Q1 unit, the more northern located bores R26/6806 (Fig. 2.4) and R26/6676 (Fig. 2.5) show a 5 - 10 $\mathrm{m}$ thick beach gravel sequence at depths of about zero and $10 \mathrm{~m}$ below mean sea level. This sequence can also be found almost $400 \mathrm{~m}$ away from the coast in bore R26/6642 (Fig. 2.5). 
However, it is clearly thinned out and the gravel layer is no longer found in the coast distal bore R26/6776 as shown in Fig. 2.5.

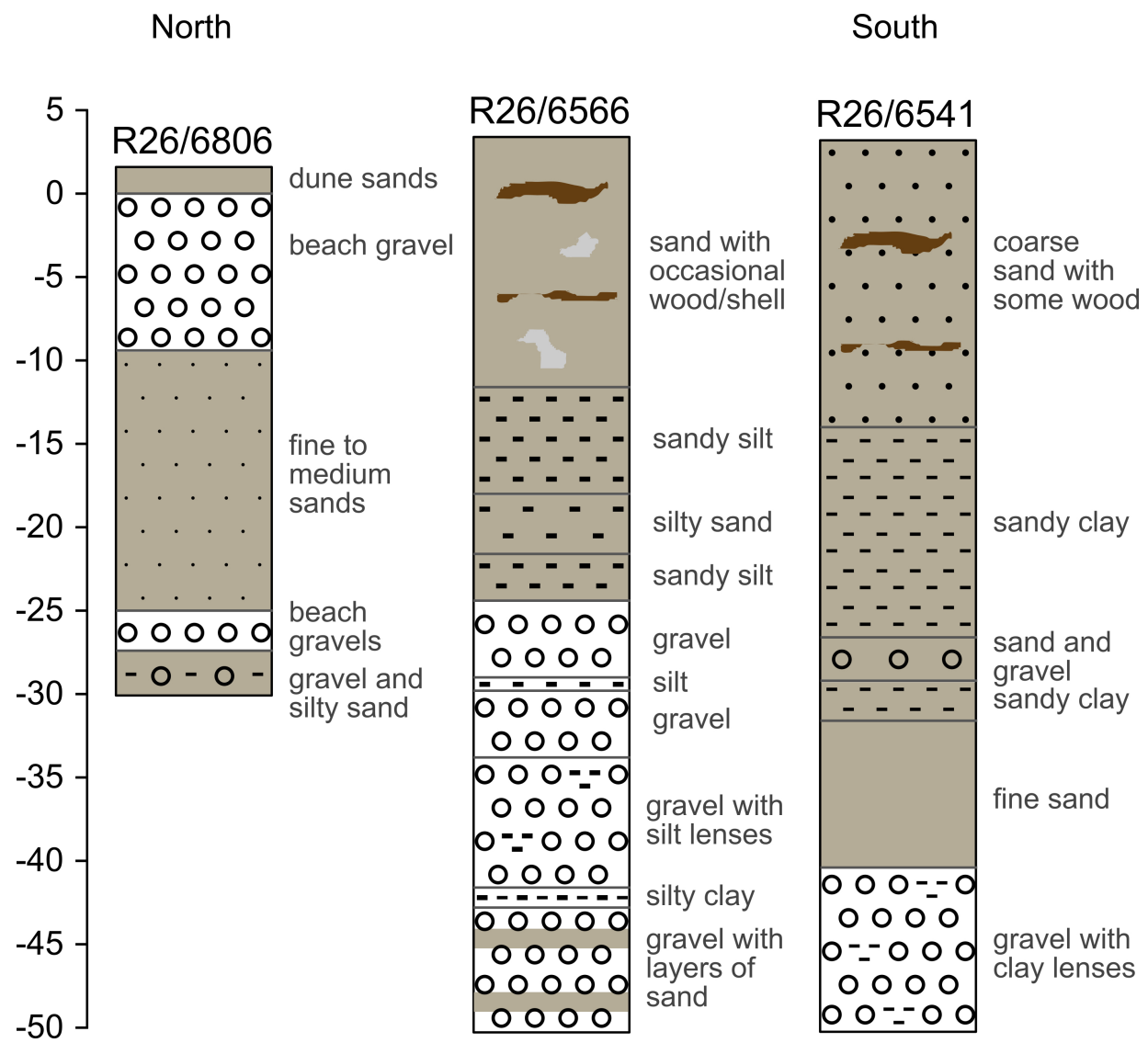

FIGURE 2.4: Bore logs obtained for three wells drilled around the Waikanae Beach area. Their location is indicated in Fig. 2.1. R26/6566 and R26/6541 information was adapted from (Morrow, 2007), whereas the one for R26/6806 was taken from (Osborne, 2006). Note that the depth is measured in meters above mean sea level.

From the geologic cross-section, constructed from three bore cores along the coast in Fig. 2.5, it is evident that the Q1 unconfined aquifer (sand, sandy gravel) extends to depths of 15 - $25 \mathrm{~m}$ below mean sea level, whereby it is thicker towards the coast. Just below is a sequence of silty sand, which likely marks the transition from the unconfined Q1 aquifer to the semi-confined Q2 aquifer (gravels from depths at around $30 \mathrm{~m}$ below mean sea level). 


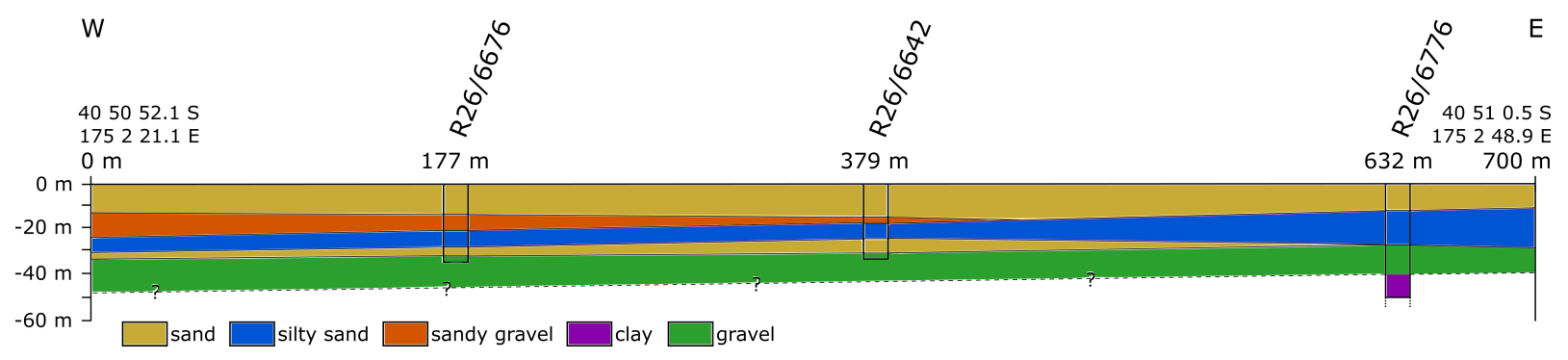

FIGURE 2.5: Geologic cross section drawn from bore information from three wells marked orange in Fig. 2.1 in the northern part of Waikanae. A more detailed location of these wells and the geological profile line is given in Fig. 4.1 (also in orange mark-up).

\subsection{Te Horo groundwater zone}

\subsubsection{Aquifer hydrology}

Although the Te Horo groundwater zone is hydrogeologically and areal-comparable to the adjacent Waikanae groundwater zone to its south, it is, with around 340 registered bores (1-192 m deep) (Gyopari et al., 2014), much less developed and researched. Due to a lack of a dense network of deep boreholes in the Te Horo groundwater zone it is even harder to accurately characterise its subsurface hydrogeology. A groundwater conceptual model for Te Horo is illustrated in Fig. 2.6. Similar to the Waikanae groundwater zone, water is found throughout all stratigraphic units. However, the three main aquifers can be identified at depth within the Q2, Q4 and Q6 units and are, according to Gyopari et al. (2014), characterised as:

1. Q2 - sand and gravel layer between 10 - $30 \mathrm{~m}$ depth with moderate permeability and more frequent gravel layers at depth

2. Q4 - gravel layer between 40 - $70 \mathrm{~m}$ depth with low to moderate permeability and increased iron concentrations

3. Q6 - poorly defined sequence with low permeability gravels between $90-150 \mathrm{~m}$ depth with elevated boron levels

At the coast these units may be found at slightly larger depths due to the slope in deposition. Generally, the water bearing layers in the Te Horo groundwater zone are associated with better sorted gravel intervals, which have possibly been reworked. The Q1 unit defines a shallow unconfined and low yielding coastal aquifer that was built up since the last 6500 years by the 
prograding shoreline (Tidswell, 2009) and consists of Holocene sands and gravels. It replaces part of the Q2 unit that has been eroded as a result of the last postglacial marine transgression (Gyopari et al., 2014) and almost directly overlies the Q2 gravel aquifer at the coast. Due to its low hydraulic conductivity of 1-2 m/day (Gyopari et al., 2014) the coastal aquifer is, like in the Waikanae groundwater zone, only used for domestic and stock water supply. From bore tests within the Q2 gravel layer close to the coast, transmissivities were found to differ by a large amount ranging from $20 \mathrm{~m}^{2} /$ day (Brown, 2003) to $2250 \mathrm{~m}^{2} /$ day (Jones, 2002). This again outlines a significant spatial variability of hydraulic properties in the Kapiti Coast aquifers most possibly related to local heterogeneities in geology. Jones (2002) also identified downward leakage from the Holocene sand aquifer during these tests.

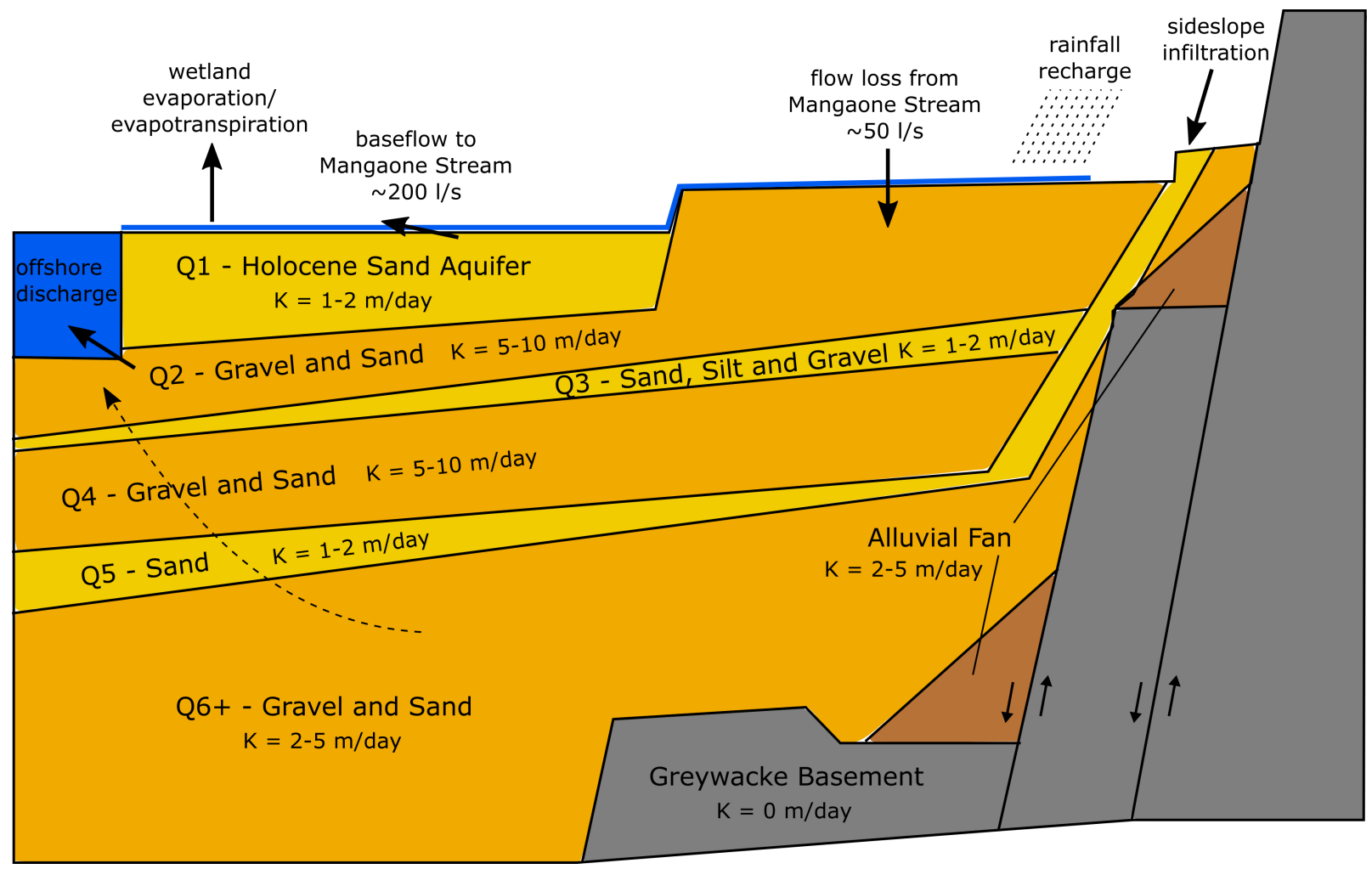

FIGURE 2.6: Schematic illustration of the Te Horo groundwater zone with the six hydrostratigraphic units (Q1-Q6), their estimated hydraulic conductivity and indicated preferential flow paths as suggested by Utting (2013) and Gyopari et al. (2014). The figure has been modified from Gyopari et al. (2014).

Recharge into the deeper aquifers is mainly by rainfall and to some extent by flow loss from the Mangaone Stream (50-200 1/s in the upper part of the stream (Gyopari et al., 2014)), whereas the unconfined shallow aquifer (Q1) shows an event driven recharge as indicated from groundwater stage and rainfall observations at the Te Hapua wetland (location see Fig. 2.1) 
as illustrated in Fig. 2.7. The Mangaone Stream originates in the Tararua Range and drains into the sea at the settlement of Te Horo Beach. Gauging measurements along the stream show consistent downstream flow loss in the upper part of the stream. This suggests infiltration of stream water into the unconfined aquifer along this section. West of State Highway 1 (SH1) this changes into groundwater seepage vertically upwards which manifests in a flow gain of $100-250$ l/s in the lower part of the Mangaone Stream. Gyopari et al. (2014) identified the sources of this flow gain to be a mixture of direct groundwater seepage and surface water runoff into the stream from artificial drains that are common in the area.

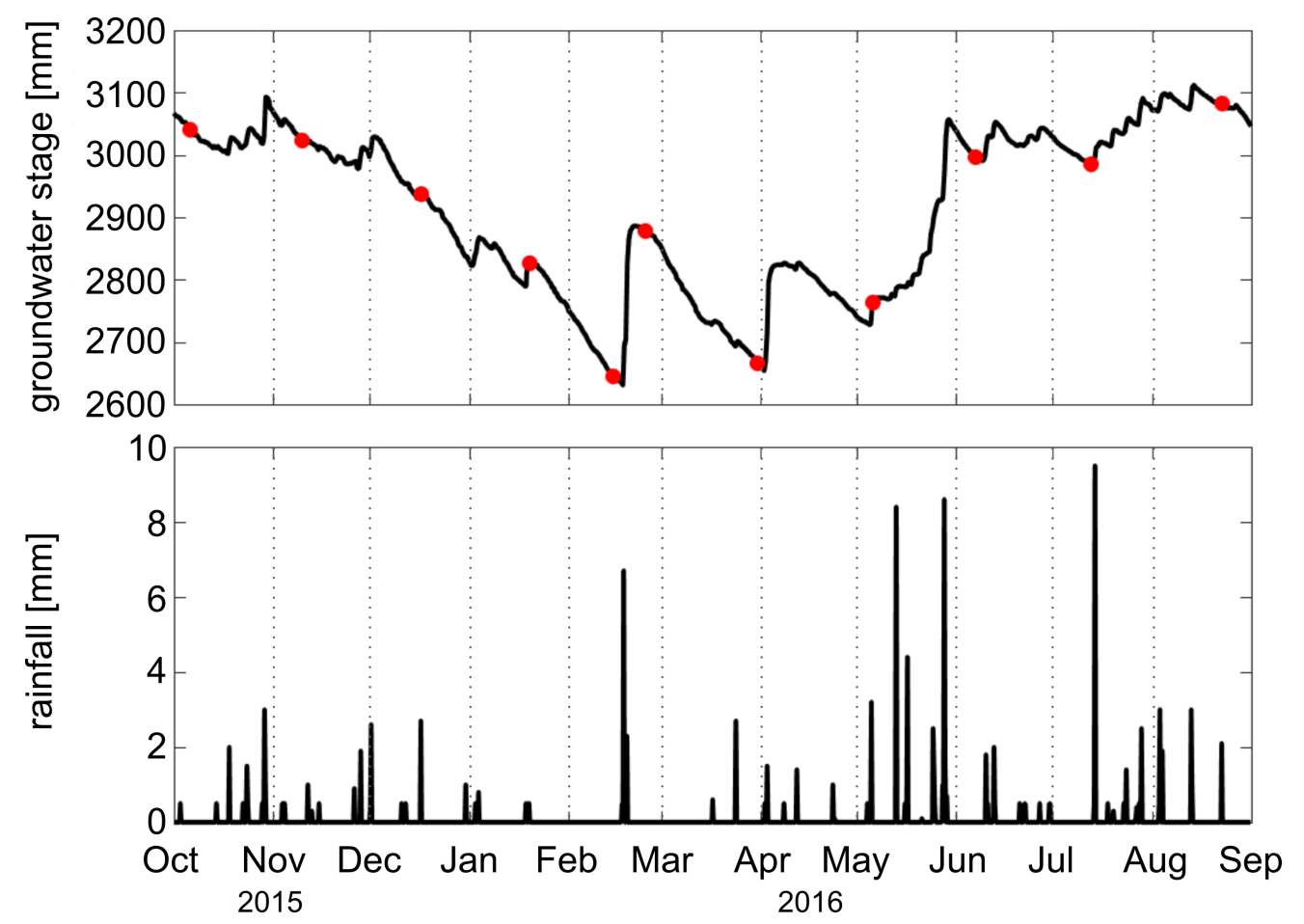

FIGURE 2.7: Groundwater stage and rainfall at the Te Hapua Wetland monitoring station (see Fig. 2.1) in Te Horo Beach (GWRC monitoring network) for the DC resistivity monitoring period in Te Horo. Red dots indicate sampling dates for the seasonal monitoring of profile THS1 (see Fig. 4.2).

\subsubsection{Coastal geology}

With a lower abundance of deep bores in the Te Horo groundwater zone it is substantially more difficult to depict the deeper sections of the aquifer stratigraphy. However, there is a reasonable amount of shallower bore log information available, which can be used to characterise the Q1 Holocene sand and gravel aquifer that makes up the top water-bearing unit within this zone 
close to the coast as indicated from the conceptual model in Fig. 2.6. As noted in Wilson (2003) the erosion rate during the Last Glacial was large and parts of the Q2 sediments were eroded. This space was later filled with the Q1 Holocene sediments during progradation since the last 6500 years as was noted by Tidswell (2009). The prograding phase is characterised by deposits of deltaic gravels, beach and dune sands, as well as inter-dunal swamp deposits (Wilson, 2003). Figure 2.8 shows two bore logs from the Te Horo Beach settlement that are both within 200 $\mathrm{m}$ from the coast. They reach depths of about 15 to $20 \mathrm{~m}$ below mean sea level and further indicate a relatively high degree of heterogeneity found in the unconfined aquifer unit along the Kapiti Coast. From these at least the uppermost about $20 \mathrm{~m}$ of the aquifer are made up from sands containing smaller or larger amounts of gravel at several depth intervals.

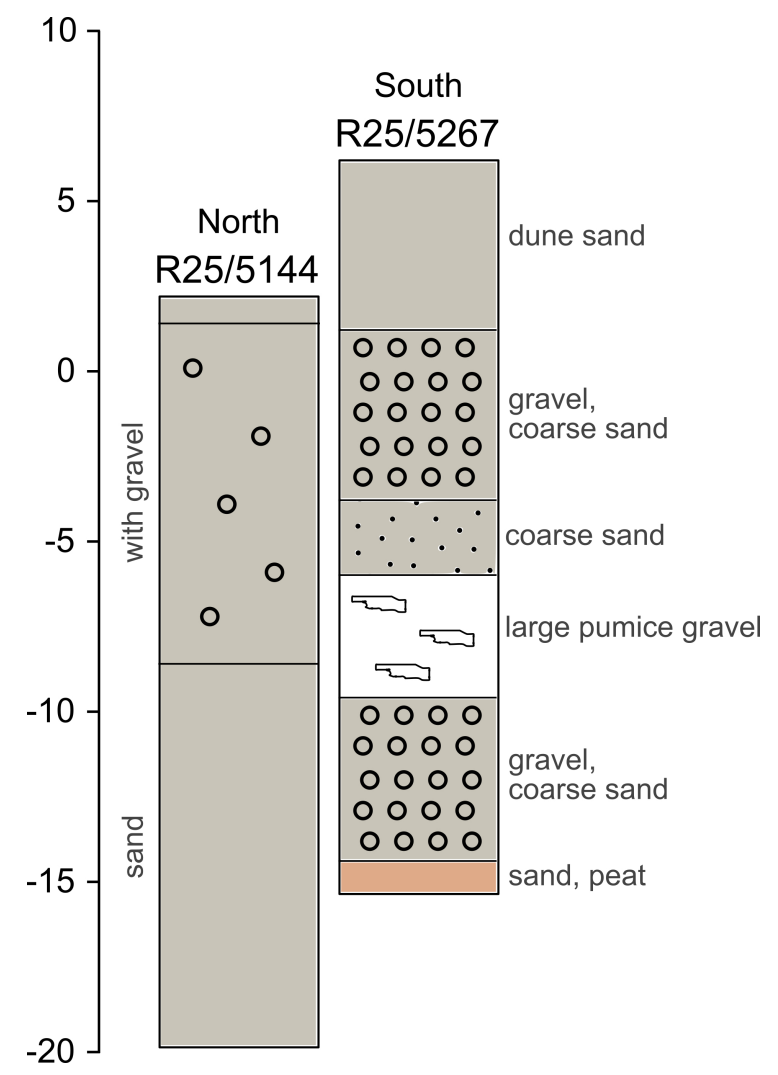

FIGURE 2.8: Bore logs obtained for two wells drilled in Te Horo Beach. Their location is indicated in Fig. 2.1 (a close-up of their location can be found in Fig. 4.2). Note that the depth is measured in meters above mean sea level.

Adapted from Wilson (2003), Fig. 2.9 shows a geologic cross section constructed from information obtained from three bore logs within $1250 \mathrm{~m}$ from the coast line. This implies that there exists a considerably thick (up to about $8 \mathrm{~m}$ ) gravel layer between the uppermost dune sand and the lower lying fine sand deposits, which varies in thickness over the horizontal extent of 
the cross section. It is interesting to note that bores R25/5144 (Fig. 2.8) and 8540043 are only about 30 m away from each other but appear to show varying depth intervals of the gravel horizon. This is another indication of the large heterogeneity of the unconfined shallow aquifer at the field site.

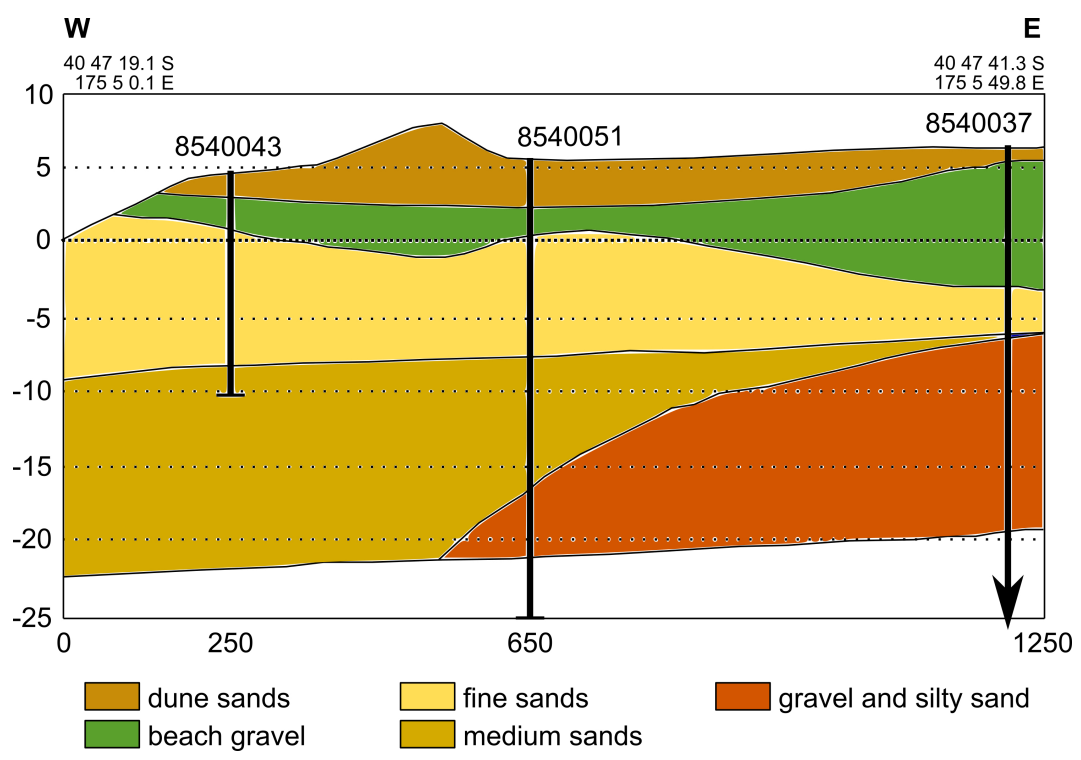

FIGURE 2.9: Geologic cross section adapted from (Wilson, 2003). Depths are in meters above mean sea level. Horizontal distances are in meters. Bore locations are marked orange in Fig. 2.1.

The only bore showing all five stratigraphic units of the about $25 \mathrm{~m}$ thick Q1 aquifer is 8540051 located approximately 650 m east to the shoreline. From Figs. 2.8 and 2.9 it appears that the shallow gravel layer continues below the Te Horo Beach township. However, with only a few bore logs available, it is not possible to conclude this with certainty. One of the most prominent differences between the Waikanae and Te Horo field sites is the occurance of the Te Horo Gravel Ridges, which cover the beach between the Otaki river mouth and the south of the Te Horo Beach settlement at the surface and up to a distance of about $80 \mathrm{~m}$ from the coast line. Whether these are only a surface occurrence or are part of the continuous gravel layer indicated in the bores of the area, is yet unclear. 



\section{CHAPTER 3}

\section{Methodologies}

"One should not increase, beyond what is necessary, the number of entities required to explain anything."

William of Ockham (1287 - 1347)

This chapter contains the theoretical background necessary to understand the data analysis in this thesis. It is organised in three sections, starting with the geophysical methods where I discuss how DC resistivity data are collected and processed with respect to this project. Then I will introduce the techniques used for the hydrological part in Chapter 6 of this thesis and finally give an overview of the two statistical approaches used for the time series analysis in Chapter 5. Specific instrumentation and survey details are given in the chapters that follow. 


\section{Geophysical}

\subsection{DC resistivity traversing and monitoring}

The direct current (DC) resistivity method (also referred to as electrical resistivity tomography (ERT) or geoelectrical method) is a geophysical technique to investigate the resistivity distribution of the underground. As the name implies, this is done by using a direct current that gets injected into the ground. In most 2D and 3D applications today, four mobile electrodes (usually stainless steel) are used for data collection. While two of them serve as current electrodes (source and sink) the other two (potential electrodes) allow for measuring the potential difference generated in the ground by the injected current. The DC resistivity method is based on Ohm's law, where the ratio of measured potential difference $(\Delta V)$ to injected current $(I)$ is multiplied with a geometric factor $(k)$, depending on the arrangement of current and potential electrodes used, to give an apparent resistivity $\left(\rho_{a}\right)$ through

$$
\rho_{a}=k \frac{\Delta V}{I}
$$

The geometric factor is determined by the arrangement (or geometry) of the four electrodes used to take the measurements. Depending on the target that needs to be investigated, the availability of space at the survey site and the labour intensity (Reynolds, 2011) the most suitable electrode geometry is chosen. With the formulation

$$
k=\frac{1}{2 \pi}\left[\left(\frac{1}{C 1 P 1}-\frac{1}{C 2 P 1}\right)-\left(\frac{1}{C 1 P 2}-\frac{1}{C 2 P 2}\right)\right]
$$

the geometric factor for any possible electrode configuration over a half-space can be calculated (i.e. borehole applications need to take a full space, and hence, image sources into account as well (e.g. Coscia, 2012)). Here, $C 1$ and $C 2$ are the current source and sink, respectively, $P 1$ and $P 2$ denote the two potential electrodes, and $C 1 P 1, C 2 P 2$, etc. symbolise the distance between the corresponding electrodes. From this it is apparent that different electrode geometries may lead to different apparent resistivity values when measured along the same survey lines. In the case of a homogeneous medium apparent resistivity is equal to the true resistivity of the ground. Heterogeneity in the resistivity of the ground leads the apparent resistivity varying for 
different electrode geometries. In order to still get an estimate of the true subsurface resistivity distribution of a heterogeneous ground, inversion techniques (Loke, 2004) are used to process the apparent resistivity data. This will be discussed later in Section 3.2. However, though using several electrode configurations can lead to an increased information content about the subsurface under investigation, this is rarely done in practice because the time necessary to do so is often not available. Hence, additional independent information about the investigated ground properties, obtained for example from bore cores, is absolutely crucial for a thorough interpretation of geoelectrical models.

Explained in physical terms, the geoelectrical method makes use of differences in how charge is conducted in different parts of the subsurface portion under investigation. This has implications on how the electromagnetic field is changed as compared to the homogeneous half-space (Knoedel et al., 2005). Good conductors will channel the electrical charges, whereas less conductive material will push the electrical charges away. The associated change in the electrical potential can be monitored, depending on the depth of the anomaly, at the earth surface. For a space-time source free case in a vacuum, the electromagnetic field is described by Maxwell's equations as,

$$
\begin{aligned}
-\nabla \times \mathbf{H}+\varepsilon_{0} \partial_{t} \mathbf{E} & =0 \\
\nabla \times \mathbf{E}+\mu_{0} \partial_{t} \mathbf{H} & =0 .
\end{aligned}
$$

In the above equations $\mathbf{H}$ refers to the magnetic and $\mathbf{E}$ to the electric field strength. Parameters $\varepsilon_{0}$ and $\mu_{0}$ are defined as the free space electric permittivity and magnetic permeability, respectively. By taking the divergence and including the presence of matter, the two equations are slightly modified to become

$$
\begin{array}{r}
-\nabla \times \mathbf{H}+\left(\sigma+\varepsilon \partial_{t}\right) \mathbf{E}=-\mathbf{J}^{e} \\
\nabla \times \mathbf{E}+\mu \partial_{t} \mathbf{H}=-\mathbf{J}^{m},
\end{array}
$$

where the material parameters are reflected through the electrical conductivity $\sigma(\mathrm{S} / \mathrm{m})$, the electrical permittivity $\varepsilon(\mathrm{F} / \mathrm{m})$ and the magnetic permeability $\mu(\mathrm{H} / \mathrm{m})$. Vectors $\mathbf{J}^{e}$ and $\mathbf{J}^{m}$ are the source volume densities of the electric and magnetic current, respectively. These equations assume instantaneous response of the earth to the electromagnetic disturbance. In the case of 
having a source of stationary electric current (i.e. at zero frequency) the general solution for the potential is the solution to the Maxwell's equations in the limit of zero frequency and given as,

$$
\begin{array}{r}
-\nabla \times \mathbf{H}+\sigma \mathbf{E}=-\mathbf{J}^{e} \\
\nabla \times \mathbf{E}=0 .
\end{array}
$$

Because the electric field in Eq. 3.8 is curl-free (i.e. time-independent), it can be expressed as the gradient of a scalar potential $V$ through

$$
\mathbf{E}=-\nabla V
$$

A differential equation of Eq. 3.7 can be obtained by taking the divergence of it and substituting the electric field with the expression in Eq. 3.9. This is known as the DC base function and is expressed as

$$
\nabla \cdot(\sigma \nabla V)=\nabla \cdot \mathbf{J}^{e}
$$

For a point source at $\mathbf{x}_{0}$ over a homogeneous half-space (i.e. in the case of a surface geoelectrical survey where the upper half-space is non-conducting air) the solution of Eq. 3.10 is given by

$$
V(\mathbf{x})=\frac{I}{2 \pi \sigma\left|\mathbf{x}-\mathbf{x}_{0}\right|},
$$

where $I$ is the strength of the electric current emitted by the source. The potential measured at a potential electrode $\left(V\left(\mathbf{x}_{P}\right)\right)$ placed at the earth surface and associated with two current electrodes of current strength $I$ at position $\mathrm{C} 1$ and $-I$ at position $\mathrm{C} 2$, is then expressed as

$$
V\left(\mathbf{x}_{P}\right)=\frac{1}{2 \pi \sigma}\left(\frac{1}{\left|\mathbf{x}_{P}-\mathbf{x}_{C 1}\right|}-\frac{1}{\left|\mathbf{x}_{P}-\mathbf{x}_{C 2}\right|}\right)
$$

where $\mathbf{x}_{P}$ represents the location of potential electrodes $P 1$ or $P 2$, respectively. The potential difference between $P 1$ and $P 2$ can then be written as $\Delta V=V_{P 1}-V_{P 2}$, which in its full form reads as

$$
\Delta V=\frac{1}{2 \pi \sigma}\left[\left(\frac{1}{C 1 P 1}-\frac{1}{C 2 P 1}\right)-\left(\frac{1}{C 1 P 2}-\frac{1}{C 2 P 2}\right)\right]
$$


By substituting $\rho=\frac{1}{\sigma}$ into Eq. 3.13 and rearranging, expression 3.1 is obtained, with the geometric factor as reflected in Eq. 3.2.

In a non-uniform subsurface at electrical inhomogeneities, the electric field causes a shift of charges and thereby the resulting electric field gets modified from its previous state (Knoedel et al., 2005). Following Snell's law, electric field lines are refracted away from the perpendicular in case of entering a good conductor, and bended towards the perpendicular when entering a less conductive layer (Reynolds, 2011) as is illustrated for a simple 2D case in Fig. 3.1. The current lines are intersected at right angles by the equipotentials with the potential decreasing inversely as the distance to the current source gets larger $(\nabla V=-\delta V / \delta x)$ (Loke, 2004; Reynolds, 2011). How the current is carried is highly dependent on the resistivity structure of the subsurface. Electric current flow in earth materials can occur through several processes: 1) the flow of free electrons (e.g. in metal), 2) electrolytic conduction (e.g. in saltwater), 3) polarisation effects (important around the electrodes), and 4) surface conduction in clays (Knoedel et al., 2005; Reynolds, 2011). Resistivity, as a fundamental property of a material, describes how much the material resists an electrical current. In contrast, the resistance of a piece of material is additionally dependent also on the size and shape of the sample ( $R=\rho \frac{L}{A}$, with $R$ being resistance, $A$ the cross-sectional area, and $L$ the length) (Reynolds, 2011). In the ground matrix porosity and permeability, the resistivity of any pore fluid, clay content, and temperature all influence the bulk subsurface resistivity. The bulk resistivity of the ground therefore depends on the conditions and this leads to large overlaps in resistivity for different rock types, as is evident by the fact that rock resistivity spans twelve orders of magnitude ranging from almost perfect insulators to almost perfect conductors (Palacky, 2006). This is one of the reasons why interpretation of geoelectric inversion models can be challenging if there is no other ground information available.

Another problem in accurate interpretation can be what is known as the principle of equivalence, which states that the product (= transverse resistance) or quotient (= longitudinal conductance) of the layer resistivity and the corresponding layer thickness can be constant for different combinations of the two (Reynolds, 2011). Therefore, several combinations of thickness and resistivity of a layer can have the same effect on the measured apparent resistivity provided their product is the same. In some cases, the subsurface might be built of three or more layers with ascending or descending resistivity with depth (Reynolds, 2011), or contain a thin layer of 
moderately lower resistivity in the middle (Albouy et al., 2001). In such a situation the middle layer might not be resolved in an inversion model because it actually does not influence the data significantly. This phenomenon is called suppression.

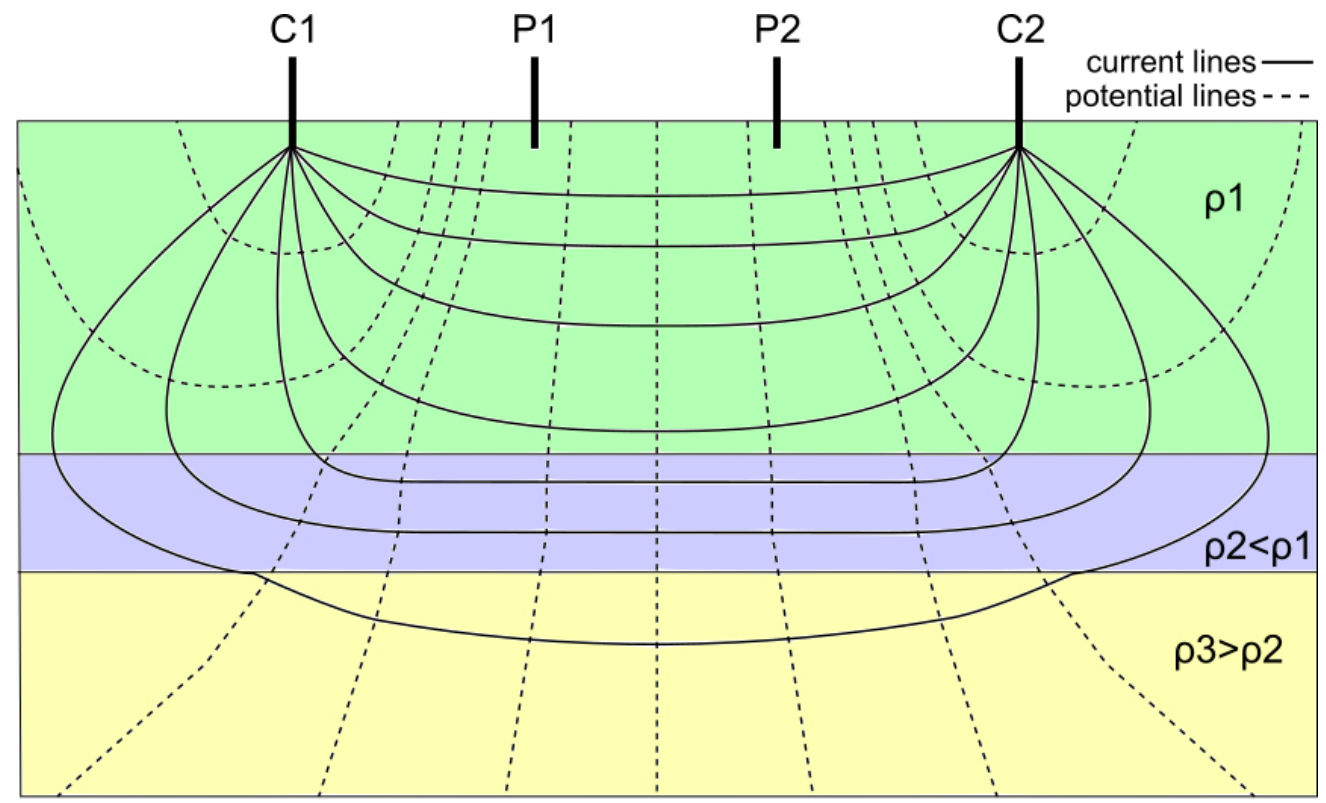

FIGURE 3.1: Basic illustration of a current flowing in the subsurface between two current electrodes ( $\mathrm{C} 1$ and $\mathrm{C} 2$ ) placed at the surface. Associated potential lines cut the current lines in a $90^{\circ}$ angle. At geophysical boundaries the current lines are bent and refracted and hence different subsurface conditions excite different potential signals measured at the surface through potential electrodes (P1 and P2).

In modern geoelectrical surveys, multi-electrode systems are used to obtain the resistivity structure beneath a profile line. This is essentially what is called resistivity traversing, profiling or tomography. The depth of penetration is mainly dependent on the profile length (the longer, the deeper - a rule-of-thumb states that the penetration depth is approximately one third to one fifth of the maximum distance between the current electrodes (Kirsch, 2006; Reynolds, 2011)) and resistivity structure of the ground. Longer profiles can be achieved by the so-called roll-along technique where electrode segments already used at the beginning of a profile line are shifted and appended to the end of it. This results in overlapping sections of the measured profile length, but does not increase the penetration depth. For monitoring purposes, the same resistivity profiles are measured several times during a defined time scale (time-lapse surveys). The changes of the subsurface resistivity over time can then be imaged in difference or ratio plots. This is especially helpful for applications where only one of the properties influencing resistivity is likely to change with time (e.g. fluid conductivity, saturation, porosity, temperature). 


\subsection{Geophysical inversion for time-lapse monitoring data sets}

Raw resistivity data are usually edited for noisy data points and then processed with geophysical inversion techniques to obtain a 'true' 2D (or 3D) resistivity distribution of the subsurface at the profile location. Over the past decades many different inversion approaches for geophysical problems have emerged, which is why the discussion of inversion theory and procedures in this thesis is limited to the ones used for data processing. Specific figures for the inversion processes will be presented in the respective chapters where the data is presented and discussed. This section only provides the theoretical background.

\subsubsection{Data editing and error estimation}

For a good quality data interpretation of DC resistivity inversion images an accurate determination of measurement errors is necessary. Noise sources can be associated with 1) poor electrode contact (systematic errors), 2) the control unit (random errors), and 3) external factors that cause background noise (sporadic errors) (Slater et al., 2000). It is customary in field surveys to repeat each measurement between about two and eight times and obtain a mean resistivity value from all these measurements (stacking). From these measurements a repetition error can then be calculated for example in form of the standard deviation (= precision estimate). However, several studies come to the conclusion that the repetition error alone is not an accurate measure of the actual error in a resistivity survey (i.e. Slater et al., 2000; Robert et al., 2011; Johnson et al., 2012). Whenever possible, geoelectrical surveys should therefore include a reciprocal set of measurements, where current and potential electrodes are interchanged to measure the same profile. In a world of no noise this should give the same resistivity value for each measurement point because the geometric factor remains the same for normal and reciprocal measurements. The reciprocal error $(\epsilon)$ can be estimated by

$$
\epsilon=R_{N}-R_{R}
$$

where $R_{N}$ and $R_{R}$ represent the normal and reciprocal resistivity value respectively (e.g. Slater et al., 2000; Robert et al., 2011). With the reciprocal error, it is possible to detect data noise that is not apparent from the repeatability error (i.e. bad ground contact of a potential electrode). For 
the data presented in this study any data points with a repetition error of more than $10 \%$ were directly deleted on data import. The data was then examined manually for outlier points, which were deleted. If reciprocal measurements were available for a data set (= measure of accuracy), the percentage reciprocal error was calculated and data points exceeding $10 \%$ of this error were culled as well. From the remaining data points a mean reciprocal error was calculated. If no reciprocal measurement were possible for a specific survey (i.e. roll-along profiles or time constraint due to incoming tides), the reciprocal error was estimated as a mean value from other reciprocal measurements made in the same area with the same instrumentation. This was then added to the mean repetition error to give a total error estimate for a resistivity data set.

In a time-lapse survey, each time step was appointed its own error estimate. If a data point was removed in any of the time steps of a time-lapse data set, this point was also removed in all of the other time steps of that data set in order to have a consistent set of measurements comparable over all time steps (e.g. Johnson et al., 2012; Kobierska et al., 2015). It is crucial that the error is a good representative of the noise in a data set because an underestimation can lead to artificial structures in the inversion model, whereas model smoothing will occur for error overestimation (Marescot et al., 2003; Robert et al., 2011).

\subsubsection{Producing time-lapse inversion models}

The principle of every geoelectrical inversion is to start from an assumed initial subsurface model (if no a priori information is available, usually the average of the resistivity data) and to calculate synthetic data based on this model and the survey measurement parameters. The field and synthetic data are then compared and the model is updated iteratively until the error between the two data sets is minimised. In order to calculate synthetic data $\left(\mathbf{d}^{f w d}\right)$, the model parameters $\mathbf{m}$ (i.e. resistivity and layer thickness) need to be related via a mathematical function F by

$$
\mathbf{d}^{f w d}=\mathbf{F}[\mathbf{m}]
$$

In the above (and following) notation, bold letters indicate vectors (lowercase) and matrices (uppercase). The expression in Eq. 3.15 is valid for a general linear forward problem. However, 
most DC resistivity (and geophysical) problems are non-linear, which requires a linearization of the expression in Eq. 3.15 by a first-order Taylor series expansion (Maurer, 2012) leading to

$$
\mathbf{d}^{f w d}=\mathbf{F}\left[\mathbf{m}_{0}\right]+\frac{\delta \mathbf{F}\left[\mathbf{m}_{0}\right]}{\delta \mathbf{m}} \Delta \mathbf{q}
$$

where $\mathbf{m}_{0}$ is the model parameter vector of the starting model, and $\mathbf{\Delta q}=\left(\mathbf{m}-\mathbf{m}_{0}\right)$ the model update vector. The term $\delta \mathrm{F}\left[\mathrm{m}_{0}\right] / \delta \mathrm{m}$ in Eq. 3.16 is commonly known as the Jacobian matrix (J) which holds the sensitivity information of the geophysical problem. The error vector $\mathbf{e}$ after incorporation into Eq. 3.16 is defined as

$$
\mathrm{e}=\Delta \mathrm{g}-\mathrm{J} \Delta \mathbf{q}
$$

where $\Delta \mathbf{g}$ is the difference between the observed and calculated data. In a least-squares inversion approach it is the goal to perturb the model parameters in $\mathbf{m}$ so that the cumulative squared error between the field and synthetic data $\left(\mathbf{e}^{T} \mathbf{e}\right)$ is minimized (Lines and Treitel, 1984)

$$
(\boldsymbol{\Delta} \mathbf{g}-\mathbf{J} \boldsymbol{\Delta} \mathbf{q})^{T}(\boldsymbol{\Delta} \mathbf{g}-\mathbf{J} \boldsymbol{\Delta} \mathbf{q}) \rightarrow 0
$$

In order to minimise the cumulative squared error, its derivative with respect to the model update vector needs to be zero $\left(\delta\left(\mathbf{e}^{T} \mathbf{e}\right) / \delta \Delta \mathbf{q}=0\right)$. The least-squares solution is then found after substituting this condition into Eq. 3.18 and differentiating with respect to $\Delta \mathbf{q}$

$$
\left(\mathbf{J}^{T} \mathbf{J}\right) \boldsymbol{\Delta} \mathbf{q}=\mathbf{J}^{T} \boldsymbol{\Delta} \mathbf{g}
$$

Any singularity (i.e. undefined mathematical object) in the matrix $\mathbf{J}^{T} \mathbf{J}$ (i.e. the matrix is not invertible) would lead to difficulties in solving the least-squares problem (Lines and Treitel, 1984). In their smoothness-constrained least-squares inversion method deGroot-Hedlin and Constable (1990) and Sasaki (1992) incorporated a Lagrange multiplier $(\lambda)$ and a roughness matrix $\Psi$ as additional constraints in order to avoid problems from singularities and unbounded oscillations (i.e. number of oscillations increases to infinity) in the solution (i.e. smoothing the parameter change vector $\boldsymbol{\Delta q}$ ) (Lines and Treitel, 1984). Including errors in the form of weighting matrices $\mathbf{W}$, this can be written as

$$
\left(\mathbf{J}^{T} \mathbf{W}^{T} \mathbf{W} \mathbf{J}+\lambda \Psi\right) \boldsymbol{\Delta} \mathbf{q}=\mathbf{J}^{T} \mathbf{W}^{T} \mathbf{W} \boldsymbol{\Delta} \mathbf{g}-\lambda \mathbf{\Psi} \mathbf{q}
$$


The roughness matrix $\Psi$ normally contains first-order difference matrices $\left(l_{2}\right.$ - or Tikhonov regularisation (i.e. errors follow a normal distribution (Menke, 2012))) but can be adjusted to other desired constraints such as an $l_{1}$-norm constraint (Loke et al., 2013) (i.e errors follow a Laplace or exponential distribution (Menke, 2012)).

Repeat 2D DC resistivity measurements along the same profile lines can be inverted in a time-lapse style in which either consecutive datasets are inverted with respect to a reference model (usually time zero) in the form of a difference inversion as suggested by LaBrecque and Yang (2000)

$$
\left(\mathbf{J}^{T} \mathbf{W}^{T} \mathbf{W} \mathbf{J}+\lambda \Psi\right)\left(\mathbf{m}_{n+1}-\mathbf{m}_{n}\right)=\mathbf{J}^{T} \mathbf{W}^{T} \mathbf{W} \Delta \mathbf{D}-\lambda \mathbf{\Psi}\left(\mathbf{m}_{0}-\mathbf{m}_{n}\right)
$$

where $\mathbf{m}_{0}$ is the reference model parameter vector and $\mathbf{m}_{n}$ the model parameter vector of time step $n$. The term $\Delta \mathrm{D}$ is a modified version of $\Delta \mathbf{g}$ that incorporates observed and calculated data vectors from the current time step relative to the reference time step

$$
\Delta \mathbf{D}=\left(\mathbf{d}_{n}^{o b s}-\mathbf{d}_{0}^{o b s}\right)-\left(\mathbf{d}_{n}^{f w d}-\mathbf{d}_{0}^{f w d}\right)
$$

Kim et al. (2009) introduced a full 4D inversion where space and time constraints are included in the inversion. This was done by using regularization along both dimensions through

$$
\left(\mathbf{J}^{T} \mathbf{W}^{T} \mathbf{W} \mathbf{J}+\lambda \boldsymbol{\Psi}+\alpha \boldsymbol{\Gamma}\right)\left(\mathbf{m}_{n+1}-\mathbf{m}_{n}\right)=\mathbf{J}^{T} \mathbf{W}^{T} \mathbf{W} \boldsymbol{\Delta} \mathbf{D}-(\lambda \boldsymbol{\Psi}+\alpha \boldsymbol{\Gamma})\left(\mathbf{m}_{0}-\mathbf{m}_{n}\right)
$$

The roughness matrix $\Gamma$ gets multiplied by a Lagrange multiplier $\alpha$ in order to control regularization in the time domain. Time-lapse inversions generally result in more stable inversion processes with less pronounced inversion artefacts generated by data noise (Ogilvy et al., 2009; Kim et al., 2009; Loke et al., 2013). The goodness of an inversion is measured by its rootmean-squared (RMS) error, which defines how well the observed and calculated data sets are matched, through

$$
R M S E=\sqrt{\frac{\sum_{i=1}^{N}\left(d_{i}^{f w d}-d_{i}^{o b s}\right)^{2}}{N}}
$$


where $N$ is the number of data points. However, a small RMS error does not automatically mean that the inversion result is the most appropriate model from a geological point of view. A good practice is to stop the inversion after the RMS error drops below the estimated noise level.

\subsubsection{Inversion model appraisal - the depth-of-investigation method}

Interpretation of geoelectrical inversion models at larger depths remains problematic due to the rapid attenuation of potential away from the current injection point. Appraisal techniques can be used to blank out the areas in a DC resistivity model that are constrained not by the field data but by the background model used in the inversion process. Often the sensitivity or resolution matrix of a model is calculated for this reason. However, not all inversion programs allow the user to do this easily. Another way of achieving resistivity model appraisal is by inverting the same dataset with two different background models (typically 0.1 and 10 times the value of the average resistivity of the dataset) and investigating which parts of the resulting inversion models agree and where they differ from each other. This technique is called depthof-investigation indexing (DOI indexing) and was first introduced for smoothness-constrained ( $l_{2}$-norm) inversions by Oldenburg and Li (1999) and further developed by Marescot et al. (2003) (scaled DOI indexing). Recently, Deceuster et al. (2014) modified this appraisal technique for robust-constrained ( $l_{1}$-norm) inversions and additionally introduced a statistically based approach to determine the cut-off factor (DOI value where the inversion model starts to be mainly controlled by the background model rather than the data - giving the maximum depth of investigation). The DOI value of an inversion model is calculated by

$$
D O I_{A B}(x, z)=\frac{q_{A}(x, z)-q_{B}(x, z)}{D O I_{M}\left(q_{A}-q_{B}\right)}
$$

where $q_{A}$ and $q_{B}$ are the two uniform resistivity background models used to perform the additional extended inversions, and $q_{A}(x, z)$ and $q_{B}(x, z)$ are the model cell resistivities obtained from these models. All resistivity values are expressed in terms of their logarithm. The two additional inversions are carried out using a model grid that extends to the ends of the survey line and to a depth of three to five times the pseudo depth of investigation, therefore ensuring that the resistivity cells at the bottom of the models are maximally dependent on the background models (Deceuster et al., 2014). DOI $I_{M}$ is the median DOI value of the deepest row of the model 
cells and is ensured to have a value representing full dependency on the background model (Deceuster et al., 2014). In order to get $D O I_{M}$, the unscaled DOI index of the bottom row of model cells needs to be calculated by using Eq. 3.25 without the division by $D O I_{M}$. Model cells that are well constrained by the data will have a DOI value close to zero (i.e. the two inversions with differing background models produce the same result), whereas those mainly controlled by the background model will approach DOI values close to one (the inversion model resistivity is similar to the background resistivity) (Fig. 3.2(a)). For the statistically obtained cut-off factor to be applicable, the DOI values need to be bimodally distributed (Fig. 3.2(c)), which is not the case for all field datasets. Two factors define if the statistical method for cut-off factor determination can be used - the bimodal distribution factor $B$ of the histogram (equal or larger than one (Pearson, 1929)) defined by

$$
B=k_{\text {urtosis }}-\text { skewness }^{2}
$$

and the Ashmann's D coefficient (Ashman et al., 1994) which calculates as

$$
D=\sqrt{2} \frac{\left|\mu_{1}-\mu_{2}\right|}{\sqrt{\left(\sigma_{1}^{2}+\sigma_{2}^{2}\right)}}
$$

with $\mu$ being the mean and $\sigma$ the standard deviations of the two Gaussian distribution functions as shown for the example in Fig. 3.2(c). Coefficient D indicates clear separation of the mixture of two Gaussian distributions for the case where it is higher than two (Deceuster et al., 2014). If the statistical approach cannot be used, then a cut-off factor has to be chosen based on where the DOI values start to increase rapidly (Oldenburg and Li, 1999), hence marking the depth of investigation. Once the inversion model (Fig. 3.2(b)) gets scaled with the DOI index with determined (statistically or manually) cut-off factor applied, only model cells that are controlled by the field data are displayed, while those mainly influenced by the background model are faded as can be seen in the example presented in Fig. 3.2(d). Hence, interpretation of the geoelectrical inversion model is solely made on parts that are considered reliable. 

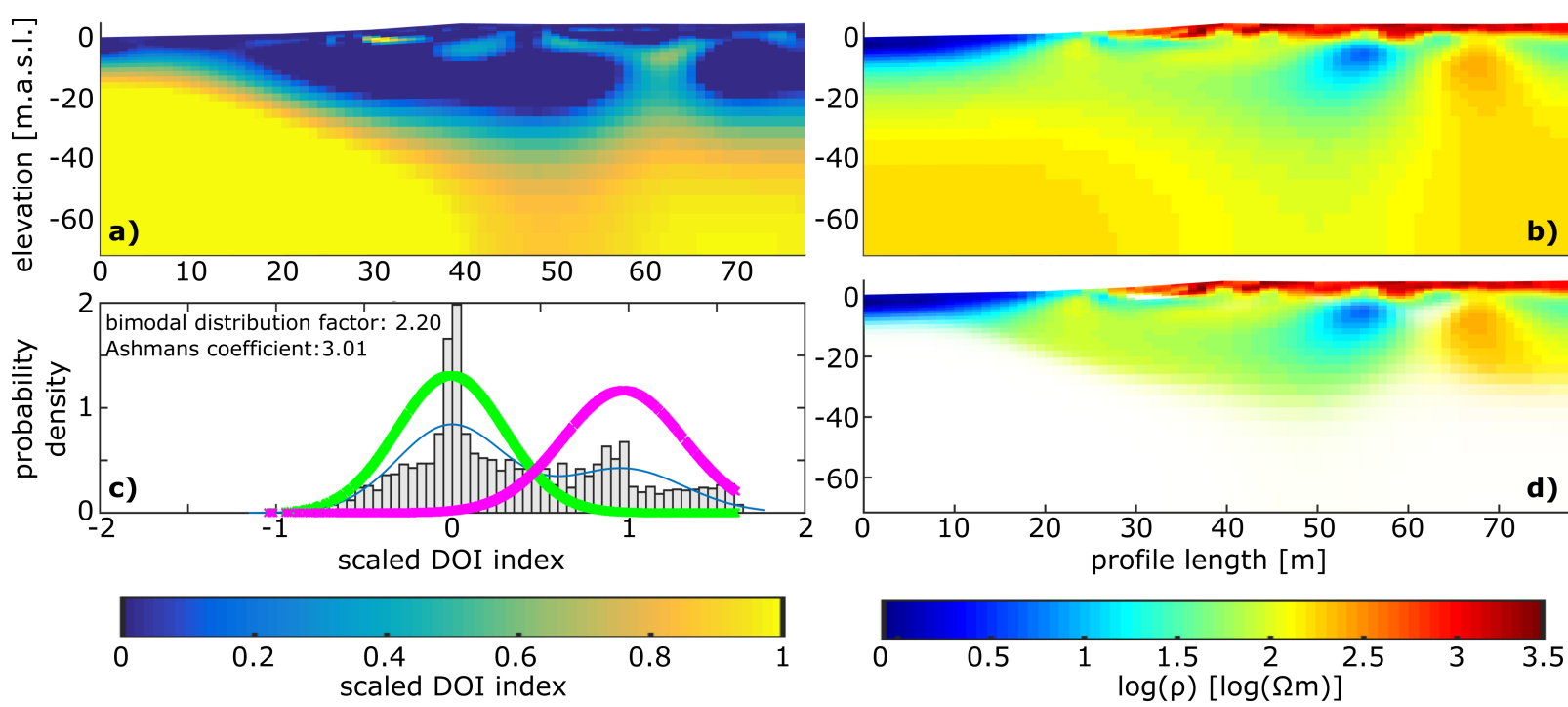

FIGURE 3.2: An example of how DOI works. a) calculated scaled DOI index for the entire model space, b) the extended inversion model using the average resistivity of the field data as a background model, c) histogram and fitted probability density function (PDF) (for each Gaussian distribution) for the cut-off factor analysis, and d) the scaled inversion model that is blending out unreliable model parts as defined by the scaled DOI index and the statistically determined cut-off factor (note: if the DOI index values would be only distributed unimodally (i.e. only one Gaussian distribution of DOI values) then a cut-off factor would need to be chosen manually). 


\section{Hydrological}

\subsection{Groundwater monitoring in wells}

Monitoring of groundwater conditions through wells and bores has a longstanding tradition in hydrology. It allows for direct measurements of groundwater composition and parameters at different depths, which is a big advantage over the geophysical methods. However, wells or bores are usually rather sparse and therefore the direct measurements are often interpolated for areas in between survey locations. This potentially introduces errors as the subsurface hydraulic properties and conditions may change within an area of investigation. Nevertheless, hydrologic information obtained from such bore measurements are valuable in hydrogeophysical investigations because they provide some sort of ground truth at certain points within a geophysical survey. With this information, geophysical data inversion can be constrained for better model interpretation. For example, knowledge of the formation factor $F$, defined by Archie's law (Archie, 1942) for a fully saturated underground through

$$
F=\frac{\rho_{b}}{\rho_{f}}
$$

with $\rho_{b}$ being the bulk resistivity of the formation and $\rho_{f}$ representing the fluid resistivity in the pores, can be used to characterise the type of fluid present in the pore matrix at a specific location. On the other hand, if the resistivity of the pore fluid is known (by, for example, electrical conductivity measurements in wells), the formation factor can be approximated for the geologic units indicated on a DC resistivity inversion image.

Groundwater monitoring seems to be a simple task, however, one should not underestimate the costs connected to design, construction, equipment and maintenance that are generated by such surveys (Western et al., 2005). Therefore, it is important that the questions about target, resolution and accuracy are well defined in advance. Most sensors that are suspended in a well record in-situ point measurements of the parameter of interest. They are normally connected to a logger system with which the measurements are recorded and stored at a pre-defined interval (e.g. every 15 minutes). The data can then either be downloaded sporadically from the logger 
TABLE 3.1: Groundwater monitoring parameters used in this thesis and how they are measured along with general resolution and accuracy information (compilation from Western et al. (2005); YSI Incorporated (2009) and Schlumberger Water Services (2010)). Underlined methods were used in this thesis and the resolution and accuracy stated are related to these methods.

\begin{tabular}{|c|c|c|c|}
\hline Parameter & Method & Resolution & Accuracy \\
\hline $\begin{array}{l}\text { groundwater level, stage } \\
{[\mathrm{mm}]}\end{array}$ & $\begin{array}{l}\text { mechanical, electronic, } \\
\text { acoustic, barometric }\end{array}$ & $20 \mathrm{~mm}$ & $\begin{array}{l} \pm 20 \mathrm{~mm} \text { or } 0.2 \% \\
\text { (whichever is larger) }\end{array}$ \\
\hline temperature $\left[{ }^{\circ} \mathrm{C}\right]$ & $\frac{\text { electrical thermometer }}{\text { (thermostat or thermistor) }}$ & $<1{ }^{\circ} \mathrm{C}$ & $\pm 0.05-0.5^{\circ} \mathrm{C}$ \\
\hline $\begin{array}{l}\text { electrical conductivity (EC) } \\
{[\mu \mathrm{S} / \mathrm{cm}]} \\
\text { salinity }[\mathrm{ppt}] \text { and } \\
\text { total dissolved solids (TDS) } \\
{[\mathrm{g} / \mathrm{l}]}\end{array}$ & $\begin{array}{l}\text { four-electrode potentiometer, } \\
\text { induction probe (salinity } \\
\text { and TDS are calculated } \\
\text { from EC) }\end{array}$ & $\begin{array}{l}0.1-1 \mu \mathrm{S} \\
0.01 \mathrm{ppt} \\
0.001-0.1 \\
\mathrm{~g} / 1\end{array}$ & $\begin{array}{l} \pm 0.5 \% \text { or } 1 \mu \mathrm{S} / \mathrm{cm} \\
\text { (whichever is larger) } \\
\pm 1 \% \text { or } 0.1 \mathrm{ppt} \\
\pm 0.5 \% \text { or } 0.1 \mathrm{~g} / 1\end{array}$ \\
\hline $\mathrm{pH}$ & potentiometric electrode & 0.1 unit & \pm 0.1 unit \\
\hline $\begin{array}{l}\text { dissolved oxygen (DO) } \\
{[\mathrm{mg} / \mathrm{l}]}\end{array}$ & polarographic, galvanic & $0.01 \mathrm{mg} / 1$ & $\begin{array}{l} \pm 0.3 \mathrm{mg} / 1 \text { or } 2 \% \\
\text { (whichever is larger) }\end{array}$ \\
\hline
\end{tabular}

in the field, or directly transmitted to a computer at a control centre. The latter allows for direct quality control but depends also on a reliable data transmitting system (modem, telemetry or radio technology (Western et al., 2005)). In return, storage capacity questions are not of primary concern as is the case with the manual download options. A major factor to consider is always the question of power requirements (i.e. to avoid loss of data). As there is usually no power available at the field sites, batteries need to be replaced at certain intervals regardless of the system. However, new technologies allow for long-term sampling with very little power use. In Table 3.1 a summary of the relevant groundwater parameters, their measurement methods, resolution and accuracy ranges is given.

Not only the spatial and temporal characterisation of groundwater measurements have to be considered when setting up a monitoring system, but also the accuracy and error of the measurement itself are important to give reliable data records (Western et al., 2005). For example, if the variation in a parameter is around $30 \%$ then a measurement error of $3 \%$ is small, whereas if the variations are in the range of $5 \%$ then this error is significant. As is the case for geophysical measurements, two types of error can occur in hydrologic monitoring; systematic and random ones. While the systematic errors normally arise from inaccurate equipment setup or calibration errors, and can be detected and possibly corrected for (Western et al., 2005), 
random errors can only be accounted for by repeat measurements (averaging). There is usually a trade-off between accuracy and resolution (i.e. either large accuracy at a small number of points or small accuracy at a large number of points) which needs to be taken into consideration as well. Some parameters (e.g. TDS, DO) can be determined very accurately in the laboratory, but this could be time and cost intensive. In contrast, it might be possible to measure the parameter of interest indirectly, but because this uses some sort of correlation the measurement accuracy will be lower due to conversion errors (Western et al., 2005). A good sampling strategy therefore should include a mixture of both.

\subsection{Chemical analysis of groundwater}

Not every parameter that provides important information for groundwater hydrology can be measured in situ. Determination of the chemical composition of groundwater belongs to this category. Knowledge of which elements are contained in what concentrations in an aquifer can serve several purposes, such as identification of the water source, water residence time or contamination of groundwater (e.g. saltwater encroachment). In the case of saltwater intrusion monitoring through geoelectrical methods, the metal content in the groundwater is especially interesting to know because these relate to electrical conductivity. In order to determine the chemical composition of groundwater, samples can be taken and analysed for their content of elements by for example mass spectrometry (Voorhees et al., 1984).

A relatively wide range of opinions exists about appropriate ways of taking a water sample so it is still representative once it gets analysed in the laboratory. However, they all agree that the samples must be treated for analysis within 48 hours of collection. Care must be taken when sampling water in the field and later analysing it in the laboratory in order to avoid contamination of the samples from outside sources, such as dirt or sweat that could lead to misinterpretations after analysis. Field sampling of groundwater is often done using a tap or pump. It is important that the pumping system is flushed with the bore water before taking the sample in order to ensure there are no residues in the pipes. Theoretically, the well needs to be pumped until one can be sure that the water running from the tap represents the groundwater from the screened well depth. This depends on how deep the screen is located and how often 
the bore is used. A water sensor can be used to monitor parameters like total dissolved solids (TDS), dissolved oxygen (DO) or electrical conductivity (EC) while letting the tap run. Once the parameters have stabilised it can be assumed that the pumping system is supplying groundwater from the screened depth and the sample can be taken. In case of a sampling site without a pump installed, the sample holder (usually sterilised PVC tubes) can be lowered about $15-30 \mathrm{~cm}$ below the water surface to take the sample. Observations such as cloudiness, colour and smell of the groundwater sample can be indications for groundwater conditions. Such observations during sampling can be an important source of information for later interpretation. It is crucial to ensure transport and storage of the samples at around $4^{\circ} \mathrm{C}$ by using a cooler in order to avoid chemical alteration of the water. Once the samples are in the laboratory they should be filtered (to remove bacteria and solid particles) and, in relation to sampling for metals, acidified with concentrated nitric acid $\left(\mathrm{HNO}_{3}\right)$ to conserve their state. The prepared samples should generally not be stored for more than 30 days (U.S. EPA, 2005) before they get analysed. Chemical groundwater analysis was undertaken using inductively coupled plasma mass spectrometry (ICP-MS) for all samples presented in this thesis. The theory behind this technique is discussed below.

\subsubsection{Hydrochemistry through ICP-MS}

Determination of elementary composition of samples through ICP-MS was first introduced in 1983 (e.g. Thomas, 2004; Wolf, 2005). It gained attention because of its superior detection limits (at or below parts per billion (ppb) level), multi-element characteristics and productivity compared to other element analysing techniques, which is why it is today the favoured method to perform trace metal analyses (PerkinElmer, 2011). Furthermore, the ability of isotopic measurements is a big benefit of the method (Thomas, 2004). But despite all the advantages that ICP-MS presents as a technique, it is rather complicated to operate and the high acquisition and running costs mean it is not always the method of choice (Thomas, 2004). The principle of ICP-MS is to ionize a sample in an argon plasma to create single-charged ions that are further separated according to their mass, and quantitatively detected.

An ICP-MS instrument is made up of two components, the inductively coupled plasma (ICP) and a mass spectrometer (MS). A schematic illustration of an ICP-MS instrument is given in Fig. 
3.3. For the plasma, normally argon is used where the gas flows in a torch that has oscillating electric and magnetic fields applied through a radio frequency (RF) coil (Wolf, 2005). This leads to a splitting of electrons from the argon atoms to form argon ions. When these ions oscillate in the fields they eventually collide with other argon atoms and thereby build the plasma (Wolf, 2005). The sample (in liquid form) is nebulized into a spray chamber before it enters the plasma. At $6000{ }^{\circ} \mathrm{C}$ the sample droplets become gaseous and by continuing their way through the plasma become highly energized and eventually release an electron in order to form a singly charged ion (PerkinElmer, 2011). Because the ions produced by the plasma are typically positively charged, elements that preferably produce negative ions such as chlorine, iodine or fluorine are hard to detect with ICP-MS (Wolf, 2005). From the high-pressure plasma chamber the ions travel through the interface cones in a vacuum into the low-pressure mass spectrometer part of the instrument. The cones have very small apertures that can become clogged if the sample has too high a content of total dissolved solids. Also, in order for the plasma to be able to ionise the sample, its TDS content should be small and approximately in the same range for comparing samples (i.e. to increase consistency and therefore accuracy). Hence, samples that are expected to be high in TDS have to be diluted prior to ICP-MS analysis (Wolf, 2005). In the mass spectrometer, the ions are separated by their mass-to-charge ratio (m/e) with a mass analyser. This way only one $\mathrm{m} / \mathrm{e}$ at the time can pass through the filter to the detector. Because different isotopes of one element have different masses, the ICP-MS method can provide isotopic information. The filter resolution of most systems is between 0.7 to 1 atomic mass unit (amu) (Wolf, 2005) and is normally sufficient to detect elements with overlapping masses. Modern systems provide the option of measuring at varying resolution (high-, medium-, and low resolution). In the detector, the incoming ions excite an electrical signal (counts per second), the intensity of which is related to the number of atoms in the sample by means of calibration standards (e.g. solution made of 1000 ppm single-element standards of all elements under investigation) (Wolf, 2005).

Instrument detection limits (IDL) for the elements of interest can be calculated as three times the standard deviation of a blank measurement (e.g. $1 \%$ nitric acid), but one should be aware that this is the best possible detection capacity of the instrument. With regard to this thesis, the two elements potassium (K) and calcium (Ca) have high detection limits, which result from mass interference of the sample matrix or the plasma species with the primary isotope (Wolf, 
detector

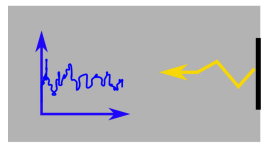

mass analyser

$\bigoplus$

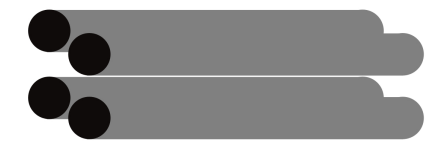

interface cones RF coil

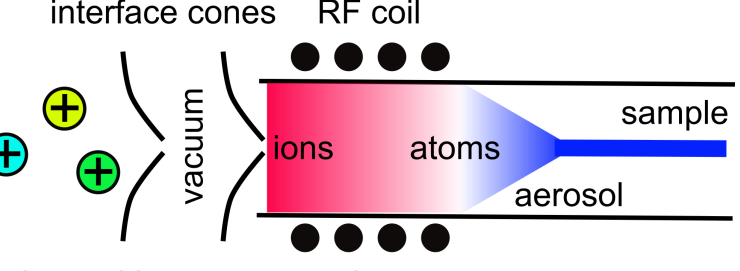

$\mathrm{m} / \mathrm{e}$ filtered ions

argon plasma

FIGURE 3.3: Schematic of an ICP-MS system. The sample gets vaporized into the argon plasma (created by a radio frequency coil that produces oscillating electromagnetic fields), where it goes from an atomic into an ionic state. At the interface between the ICP and the MS systems, the positively charged ions travel through interface cones that are connected by a vacuum chamber in order to allow for pressure differences between the two instrument parts. An electrostatic filter (e.g. quadrupole mass filter or sector field filter) only allows to pass ions with a specific mass-to-charge ratio which is defined by the measurement sequence and changes over time so all elements of interest can be measured. Then the filtered ions get counted through the intensity of the electric signal in the detector they excite (proportional to the number of ions in the sample).

2005). Less abundant isotopes of these elements should therefore be used to avoid this problem, and measuring in high mass resolution mode can also help detecting the correct mass. The system is operated and monitored by a computer where the user has to define the sequence of measuring standards, blanks and samples and needs to keep an eye on instrument parameters in order to ensure proper operation.

The instrument provides data either in raw form, as counts per second (cps) of the masses measured, or already converted into parts per billion (ppb). In order to get from cps to $\mathrm{ppb}$ the operator (or machine) has to perform a series of calculations. First, the blanks (e.g. $1 \%$ $\mathrm{HNO}_{3}$ ) have to be subtracted from the calibration standards and samples. This will remove the background concentration and therefore noise. Odd looking (i.e. very high counts) blank measurements can be used as indicators for measurement errors and such blanks should be omitted for background subtraction. Because the operator knows the correct concentrations of the elements $(j)$ in the calibration standards $\left(p p b_{\text {calib.std }}\right)$ and the dilution factors of the samples $(D F)$ (in case they needed to be diluted), these can be used to calculate sample concentrations $\left(p p b_{\text {sample }}\right)$ from their cps values (cps sample $)$ in a second step by

$$
p p b_{\text {sample }, j}=\left(c p s_{\text {sample }, j} /\left[\frac{1}{n} \sum_{i=1}^{n} \frac{c p s_{\text {callibstd }, i j}}{p p b_{\text {calib.st }, i j}}\right]\right) * D F_{\text {sample }, j}
$$

where the denominator in Eq. 3.29 is the average of all calibration standards measured in a sequence. Precision of the analysis is estimated by two standard deviations of the secondary 
standard. The secondary standard river water used here, certified reference material SLRS-4 (Yeghicheyan et al., 2001), allows calculation of a bias between the measured and the expected concentration of that secondary standard. If the bias looks suspiciously regular throughout all elements, this can be an indication for a systematic error in conversion calculations. Also, elements that show a bias exceeding 10 \% should be treated cautiously. From the blanks the detection and quantification limits are calculated as three and six times the standard deviations of all blank measurements in a sequence, respectively. Sample values that fall below the detection limit should be ignored for analysis. Elements with values that lie in between the detection and quantification limits, although contained in the sample, have a concentration that cannot be properly defined. Further error analysis is done by analysing the average bias for each element $j$ between the measured and known calibration standard concentrations defined by

$$
\text { Bias }_{j}=\frac{1}{n} \sum_{i=1}^{n} \frac{\left|p p b_{\text {calib.std, } i j}^{\text {measured }}-p p b_{\text {calib.std }, i j}^{\text {known }}\right|}{p p b_{\text {calib.std }, i j}^{\text {measured }}} 100
$$




\section{Time-series analysis}

\subsection{Dynamic Time Warping for measuring signal time lags}

In terms of studying seawater mixing dynamics tied to cyclical processes, like tidal wave run up at the beach face, it is beneficial to somehow investigate the correlation of these two processes. It is intuitive to expect that if the processes are interrelated that there would be a time lag between their oscillating signals. In such cases, often the cross-correlation of the two discrete time sequences is computed in order to find the shift between them in either space or time. However, this technique is unsuitable for the data presented in this thesis for the reason that the two signals under investigation do not show the same period (i.e. it takes longer for the mixing within the aquifer to adjust to changes than the tidal stage going through a full cycle). A better measure of time series similarity for such data sets is posed by dynamic time warping (DTW), which analyses the entirety of the temporal nature of a signal and finds the optimal global alignment of the two sequences (Petitjean et al., 2012). In 1978 Sakoe and Chiba (1978) first developed this dynamic programming solution for the use in speech recognition (Hale, 2013), but it has since been applied to many other time series analysis problems in various fields. An example to illustrate the difference for similarity search in time series between cross-correlation and DTW is given in Fig. 3.4.

DTW gives an overall number which quantifies the similarity between the two time series and can be understood as a distance in time (or extent). If the two time series are termed $X$ (i.e. tidal stage) and $Y$ (i.e. model cell conductivity) with lengths $N$ and $M$, then the distance matrix $c[N \times M]$ holds the distances for each point $X_{n}$ and $Y_{m}$. This distance can be calculated as the Euclidean distance for each element in the $(N x M)$-matrix as

$$
c\left(X_{n}, Y_{m}\right)=\sqrt{\left(X_{n, l}-Y_{m, l}\right)^{2}}
$$

The principle behind DTW is to constrain the rate at which shifts vary in time, which makes it a very accurate and powerful tool for cases where the two signals are not just simply time shifted copies of each other (Hale, 2013) and/or do not have the same length. First, a cost 


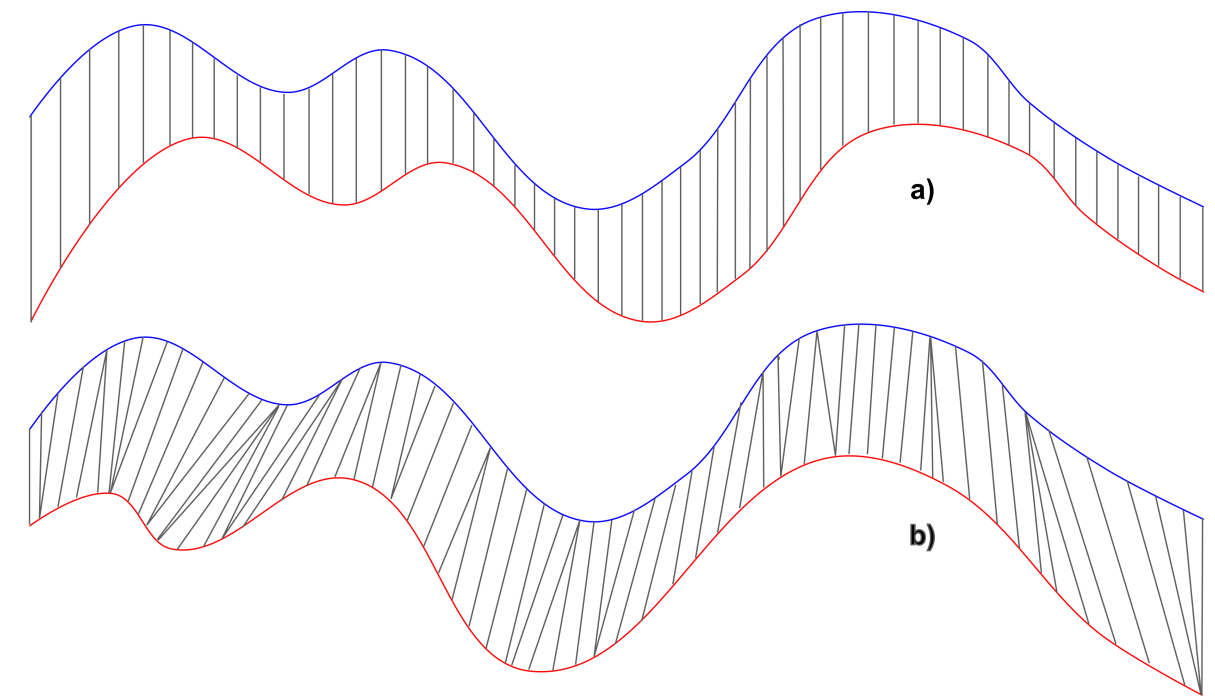

FIGURE 3.4: Difference of similarity (distance) measure between a) cross-correlation of two similar signals (blue and red) shifted in time, and b) dynamic time warping (DTW) of two similar signals with different temporal behaviour.

matrix $D[N \times M]$ is pre-conditioned by the cumulative sum of the distances between $X$ and $Y$ in $N$ and $M$ directions. The cost of the optimal alignment (i.e. the optimal warping path) is then recursively calculated using

$$
D(n, m)=c\left(X_{n}, Y_{m}\right)+\min \left\{\begin{array}{l}
D(n-1, m-1) \\
D(n-1, m) \\
D(n, m-1)
\end{array}\right.
$$

An example of the optimal warping path for a tidal stage and model cell conductivity time series is illustrated in Fig. 3.5. It follows the path of minimum cost through the recursively computed $(N \times M)$-cost matrix, where the element $D(N, M)$ marks the overall similarity or separation of the two signals under investigation. This can be calculated for each model cell in a DC resistivity inversion model in order to find the optimal warping cost (OWC) between tidal and resistivity signals. This can be understood as a similarity measure between the two signals and the smaller the number, the more correlated the signals are, with an OWC of zero reflecting identical signals. 


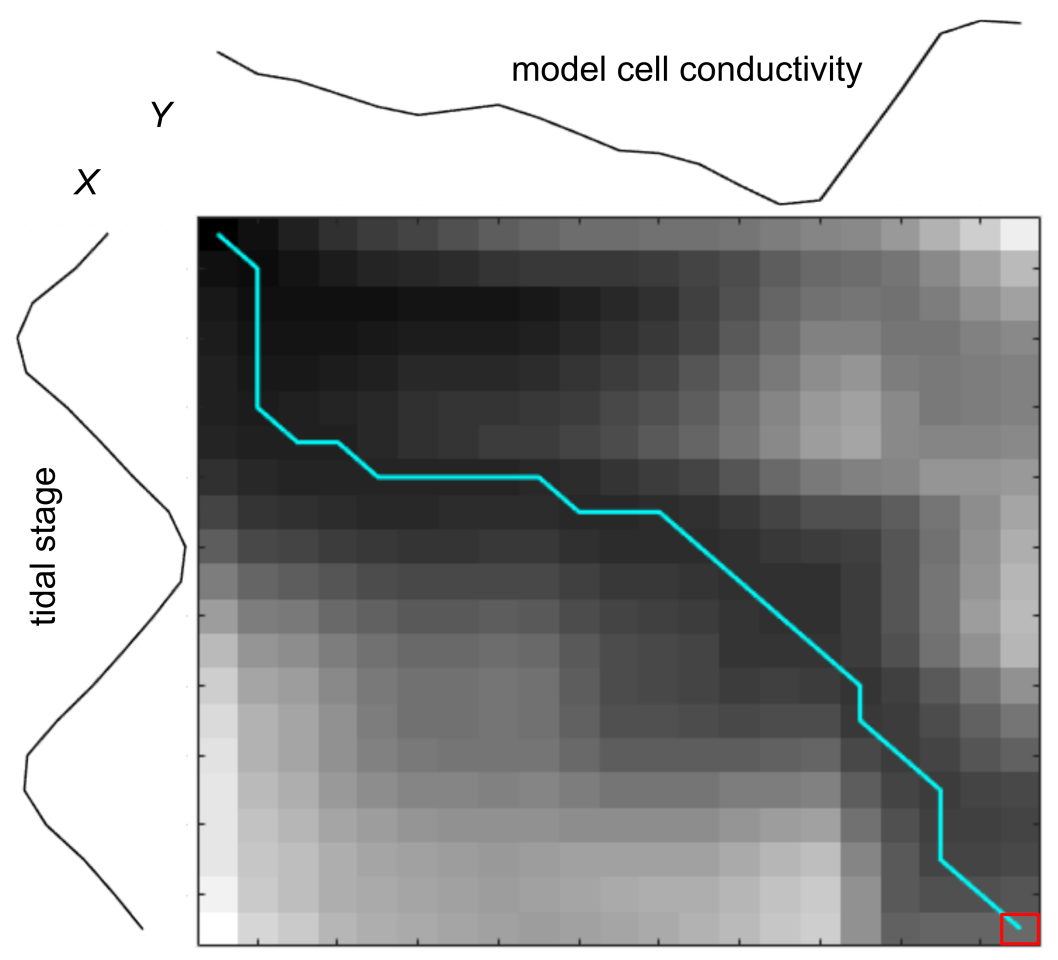

FIGURE 3.5: Cost matrix D with optimal warping path (blue) for a dynamic time warping (DTW) analysis of the tidal stage signal (X) and a model cell conductivity time series (Y). The red rectangle marks cell $\mathrm{D}(\mathrm{N}, \mathrm{M})$ which represents the overall similarity/separation of the two signals.

\subsection{Autocovariance for periodicity retrieval in time series}

Analysing coastal aquifer DC resistivity monitoring data sets for recurring patterns may yield information about natural oscillating processes that influence seawater intrusion into the aquifer. The time it takes to go through an entire cycle (e.g. from lowest resistivity to highest resistivity to lowest resistivity, or high tide to low tide to high tide) is expressed by the period of a signal. This period can be obtained by performing a cross-correlation of the mean $(\mu)$-removed sequences of the signal with itself at different time lags - the autocovariance. It is a measure of dependence between the time series $x$ and its time shifted copy $y$ at several time lags $L$, and is defined by

$$
\operatorname{cov}_{x y}(L)= \begin{cases}\frac{1}{N} \sum_{t=1}^{N-L}\left(x_{t}-\mu_{x}\right)\left(y_{t+L}-\mu_{y}\right) & L=0,1, \ldots, N-1 \\ \frac{1}{N} \sum_{t=1}^{N-L}\left(x_{t+L}-\mu_{x}\right)\left(y_{t}-\mu_{y}\right) & L=-1,-2, \ldots,-(N-1)\end{cases}
$$

where $N$ is the length of the time series. The Auto-covariogram of a 21-hour tidal stage signal at the field site in Te Horo is displayed in Fig. 3.6 and illustrates the characteristics of such 
analysis.

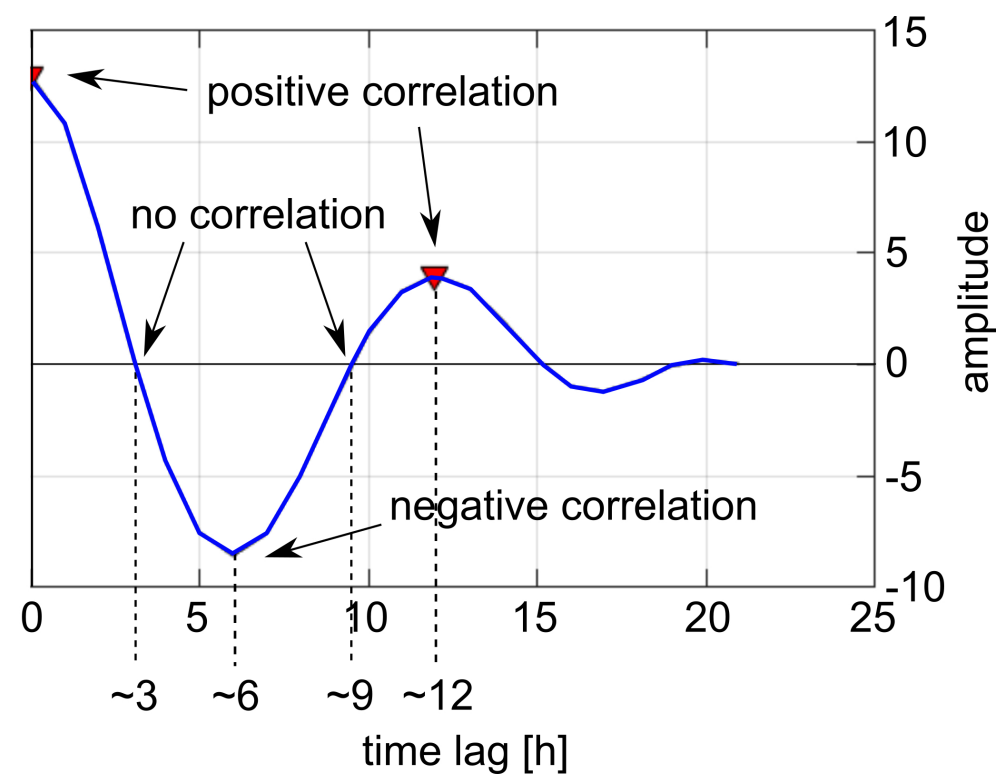

FIGURE 3.6: Auto-covariogram of a 21-hour tidal signal at the field site in Te Horo. It becomes clear that the zero-amplitude crossings mark the time between the tidal extremes every six hours (no correlation of the signal with itself), whereas positive correlation is achieved approximately every twelve hours (i.e. the period of the signal). Negative correlation occurs roughly six hours after a positive correlation (i.e. low vs. high tide stage) and its recurrence time is also about twelve hours.

Positive correlation between the original sequence and its time shifted twin is indicated by the positive peaks, and vice versa for negative correlation. The time lag between these peaks gives the period of the signal (i.e. the time it takes until the same state in a signal is reached), which is around 12 hours for the tidal time signal analysed in Fig. 3.6 and therefore characterises the time it takes to go through an entire tidal cycle (i.e. low tide to low tide). In theory, a complete tidal cycle at a semi-diurnal tide location (i.e. the case at the field sites discussed herein) takes 12 hours 25 minutes (or half a lunar day), which is slightly longer than the result of the autocovariance of the tidal signal analysed here. However, because the sample interval is set at one hour for all resistivity signals analysed with this method, it is not expected that the period of these signals are resolved within such accuracy and therefore an approximate 12-hour tidal cycle is thought to be a fair reference. Where the autocovariance function crosses the zero-amplitude mark, no correlation between the signal and its time shifted copy is found and corresponds to the times between tidal extremes (i.e. between low and high tide) in the above example. The peak-to-peak or cycle time can easily be found by determining the positive 
peak positions in the auto-covariance function for a time series, and then taking the mean of the differences between all adjacent peaks. 



\section{CHAPTER 4}

\section{Seasonal time-lapse geoelectrical monitoring of salinity changes in shallow coastal aquifers}

"The greatest threat to our planet is the belief that someone else will save it." Robert Swan, first person to walk to both Poles (1956 - present)

Part of this chapter (Waikanae groundwater zone Section 4.4.1) was published in Near Surface Geophysics: E. Sutter and M. Ingham (2017). Seasonal saline intrusion monitoring of a shallow coastal aquifer using time-lapse DC resistivity traversing. Near Surface Geophysics 15, 59-73. A higher time resolution study with similar purpose was carried out during the publishing process and its results and implications are added to this chapter. Then I draw conclusions related to saltwater intrusion into the shallow coastal aquifer based on the two seasonal monitoring studies. 


\begin{abstract}
Long-term time-lapse DC resistivity measurements at two locations across a shallow coastal aquifer on the Kapiti Coast in New Zealand have been carried out to image seasonal changes in its resistivity distribution. Along rurally located profiles repetition of the measurements shows a continuous decrease in the bulk resistivity at depth over an extended horizontal portion of the profile lines when coming into the drier summer season. This is interpreted to be the result of a decrease in the fluid resistivity resulting from lower freshwater pressure that allows saltwater to intrude. Coming into the wetter winter season, the bulk resistivity at depth increases again as freshwater pressure in the aquifer increases. Resistivity transects that were positioned in the vicinity of residential areas, however, indicate the opposite behaviour within a seasonal time frame. The dominance of other processes such as evapotranspiration and groundwater stage, compared to those being in control in a natural environment (freshwater/seawater pressure relationship) are suspected to be responsible for these observations, due to differing underground circulation patterns between rural and urban areas. Independent of each other, the two long-term seasonal saline intrusion monitoring experiments have shown significant changes in resistivity, correlated to the amount of freshwater available at any time, during the course of a year. Time-lapse monitoring using electrical resistivity tomography has thus proven itself as a suitable tool for imaging both the saline interface shape and extent and the changes in the subsurface resistivity distribution in an unconfined coastal shallow sand aquifer over a seasonal time frame. The results significantly add to the current knowledge about the dynamic behaviour near the saline interface in coastal aquifers and can be used for groundwater assessment in this and other similar geological and hydrological environments around the world.
\end{abstract}




\subsection{Introduction}

Aquifers bounded by the sea are dynamic environments influenced by various factors such as climate variations, groundwater pumping, and fluctuating sea levels (Werner et al., 2013), and they are prone to saltwater intrusion. Problems in terms of saltwater intrusion into freshwater supplies of coastal zones occur for different reasons, of which excessive over-pumping is the main anthropogenic cause. Furthermore, the saltwater wedge can shift a considerable distance inland when the outflow of freshwater is decreased, such as by reducing natural aquifer recharge zones (Oude Essink, 2001).

The boundary between the underground stored salt- and freshwater in a coastal aquifer is termed the saline interface. This interface and its limit and extent (the mixing zone - a more or less extensive transition zone between the two fluids) are controlled by various factors such as freshwater flow through the aquifer, thickness and hydraulic properties of the hydrogeological units, and the density ratio of the two fluids (Barlow, 2003). In theory, freshwater overlies saltwater due to its lower density (Fig. 1.1), and the fresh-saline interface can be roughly described by the Ghyben-Herzberg relationship (Badon Ghyben, 1889; Herzberg, 1901). However, field observations often do not match the theoretical expectations because there is an almost infinite array of possible hydrological and geological settings that influence saline intrusion (Werner et al., 2013).

Increased private and commercial use of bore water on the Kapiti Coast in New Zealand has raised concerns about saline intrusion into the shallow aquifers of the area. Efficient groundwater management is essential to secure freshwater supply in coastal regions because the underground stored water is often the only source of freshwater in these areas (Oude Essink, 2001). It is important that countermeasures are undertaken before the effects of saltwater intrusion are apparent. This requires accurate knowledge of the processes leading to saline intrusion, and hence, knowledge gaps need to be filled. Werner et al. (2013) recognised that the biggest shortfall in saline intrusion research is in the lack of extensive field monitoring studies on the mixing zone changes for different hydrogeological situations and their relation with hydrological prediction models or hydrological field data. 
A coastal aquifer with a high hydraulic conductivity and permeability is expected to quickly react to changes in its parameters such as the total dissolved solids (TDS) abundant in its stored water. If seawater was to intrude such a type of aquifer, then an immediate increase in TDS could be expected. Vice versa, with increasing freshwater pressure (for example after a period of rain) the TDS content would be decreased due to dilution of the salinised groundwater. In places where rainfall and draught are seasonally driven (i.e. show an oscillating seasonal pattern with time) it could be expected that seawater intrusion (SI) in the shallow aquifers follow a similar pattern if freshwater pressure was to be the driving force of SI in that area. In order to predict anthropogenic influences (disturbances) of a natural equilibrium, the natural processes influencing this equilibrium must be understood first. Therefore, the scope of this long-term monitoring study is to image a seasonal cycle of saline intrusion, using repeated DC resistivity measurements to provide a tool for future management of groundwater in the study area and to contribute towards filling knowledge gaps related to saline intrusion in general.

The methods used for data collection, editing and processing are explained in Sections 3.1 and 3.2. A first pilot study for seasonal SI monitoring with geoelectical methods was conducted in Waikanae Beach along six profiles in a two-monthly cycle for the period of one year. This was then repeated with a better time resolution and at a different field site in Te Horo Beach (15 km north) in order to confirm the observations from the first study and show eventual discrepancies. In the following section the two field sites are introduced and the parameters used to collect the geoelectrical data sets are discussed. Then inversion strategies are explained before the results of each field site are presented. Finally, the discussion of these results will lead to conclusions for seasonally driven SI processes in the area of investigation.

\subsection{Field sites and geoelectrical measurements}

In order to study seasonal patterns in SI with repeat DC resistivity measurements, two field sites along the Kapiti Coast were chosen (Fig. 4.1 for Waikanae and Fig. 4.2 for Te Horo). The hydrogeology of the area is thoroughly discussed in Chapter 2. Because the two surveys differ in some parameters, Table 4.1 gives an overview of these for each field site. 
TABLE 4.1: Survey parameters for the two DC resistivity monitoring sites. The positions of the monitoring profiles are indicated in Figs. 4.1 and 4.2 for the Waikanae and the Te Horo groundwater zones, respectively.

\begin{tabular}{l||cc}
\hline Groundwater zone & Waikanae & Te Horo \\
Number of DCR profiles & 6 & 3 \\
Length of profiles [m] & $145-275$ & 155 \\
Repetition period for DCR measurements [days] & 60 & 30 \\
Monitoring duration & Aug 14 - Jun 15 & Oct 15 - Aug 16 \\
\hline
\end{tabular}

All profile lines ran perpendicular to the coast line. Their horizontal extension was subject to space, limited by private properties in the southeast and the sea in the northwest, and measured $145 \mathrm{~m}$ for S3; $155 \mathrm{~m}$ for S1, S2, and N3; $235 \mathrm{~m}$ for N2; and $275 \mathrm{~m}$ for the northernmost profile N1 in Waikanae (Fig. 4.1). The profiles in Te Horo Beach (Fig. 4.2) all were $155 \mathrm{~m}$ in extent. Measurements were conducted with an ARES multi-electrode resistivity meter (GF Instruments, CZ) and 5-m minimum electrode spacing using the Wenner- $\alpha$ array.

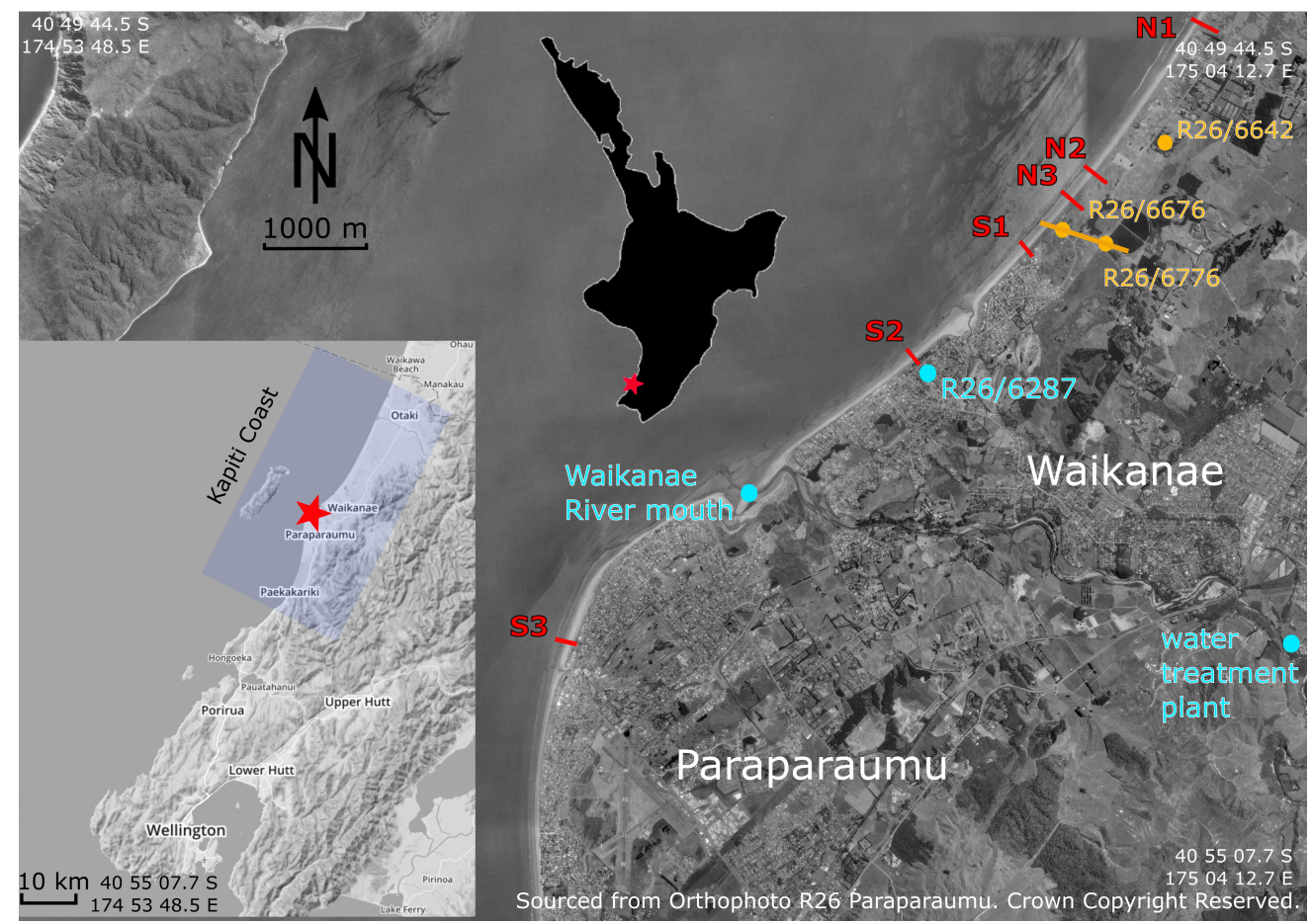

FIGURE 4.1: Map of the study area around Waikanae (Kapiti Coast (NZ)). Indicated in red are the six DC resistivity transects, blue points mark groundwater table level a GWRC monitoring well and the Waikanae water treatment plant, whereas orange points show where the geologic bore $\log$ information was obtained. AA' marks the geologic section drawn from the bore information (Fig. 2.5).

After several test measurements along the profile lines prior to the start of the first monitoring experiment in Waikanae, this geometry was found to be superior to other array geometries (i.e. 
dipole-dipole, gradient) in terms of signal strength for imaging the saline interface. Two 5-m minimum spacing time-lapse measurements made over a span of ten hours each indicated that there was no significant ( $>5 \Omega \mathrm{m}$ ) change in the resistivity response apparent for this spacing. Hence, an influence of the tides on the seasonal resistivity changes would be avoided at this spacing, which was anticipated. The first two to eight electrodes (up to 35-m profile length) were usually placed in the seawater using 1.2-m-long stainless steel rods to keep the electronics above water. Images showing the general field set up inland and on the beach are shown in Fig. 4.3. For repeat measurements, to keep the electrode position deviation from time step to time step as small as possible, the electrode positions were recovered by locating a single fixed position (usually at the end or middle of the profile) that was well constrained by a prominent landmark. From this point, the electrode layout was constrained by the 5-m takeout of the cables and by checking the positions of every eighth electrode with GPS and further orientation on local landmarks. The resulting error in electrode placement is estimated to be less than one meter. Electrode shifts during time-lapse surveys can lead to artefacts in the time-lapse inversion (Zhou and Dahlin, 2003; Wilkinson et al., 2010). However, as Zhou and Dahlin (2003) showed, the Wenner- $\alpha$ array is rather insensitive to spacing errors. Measurements with deliberately misplaced electrodes along the TH3 profile in Te Horo Beach (see Fig. 4.2) showed that for the profile measured with a Wenner- $\alpha$ array and a 5-m initial spacing, the overall percentage error introduced by measuring with electrodes shifted in different directions by a maximum of one meter is $0.75 \%$. This is smaller than the percentage error $(2.97 \%)$ resulting from a reciprocity measurement of the initial profile. Hence, it was concluded that electrode misplacements of at least up to $1 \mathrm{~m}$ are not resolved in the data.

To obtain accurate $( \pm 0.1-0.5 \mathrm{~m})$ topographic information, each profile was measured with a Trimble GeoExplorer Differential GPS. This information was included in the time-lapse inversion procedure and allows the inversion process to correct for deviations from flat topography. During summer months in Waikanae (from December 2014 to March 2015) and all-year round in Te Horo, electrodes placed on the dry loose dune sand and gravel had to be watered with seawater to allow current to be injected into the ground and ensure good quality of the field data (electrode resistances of less than $1500 \Omega$ ). For the first monitoring survey in Waikanae measurements were also timed accordingly to tide heights so that tidal influences on the saline intrusion monitoring could be minimised. A sea level height of approximately one meter above 


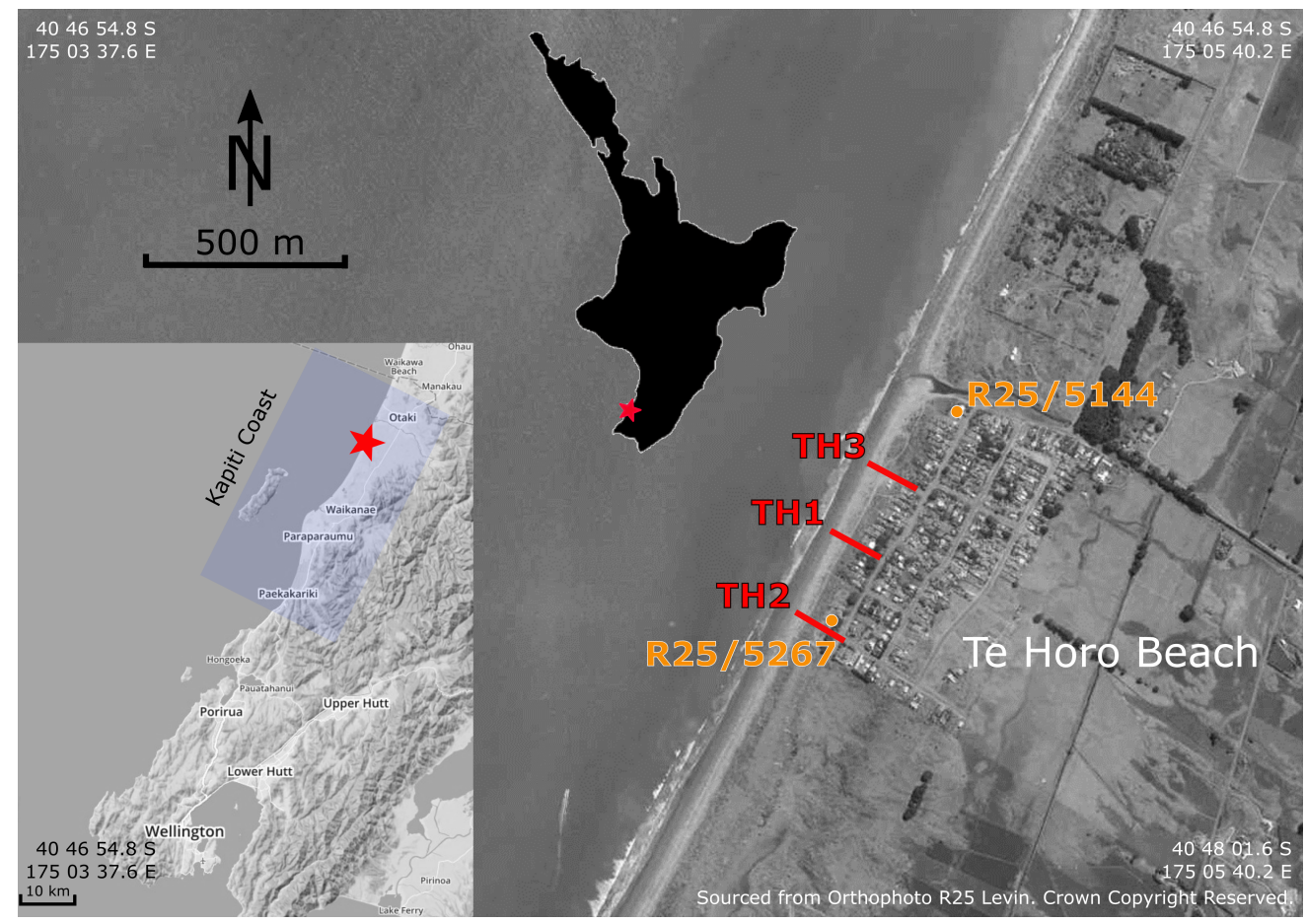

FIGURE 4.2: Map of the study area around Te Horo Beach (Kapiti Coast (NZ)). Indicated in red are the three $155 \mathrm{~m}$ long DC resistivity transects that were monitored in a roughly 30-day cycle during 2015/2016. North of the settlement the Mangaone Stream is discharging into the sea.

the lowest possible tide at the Waikanae River mouth was chosen as a compromise between suitable (daylight) measurement times and possible seaward profile extension. However, due to variations in wave action, this was difficult to maintain at all times, and effective seawater levels during the measurements are therefore indicated on the inversion profiles. As there were no indications from the pilot study in Waikanae for tidal dependencies of the seasonal monitoring (as further discussed later in Sections 4.4.1 and 4.5), the Te Horo measurements were timed to be within 2 hours from low tide in order to allow for maximum transect extent. Seawater level was not taken into account in the inversion process as, for example, proposed by Henderson et al. (2010), because the minimum spacing of $15 \mathrm{~m}$ between the current source and sink gave no reason to assume that the current would preferentially flow in the seawater over this distance. 

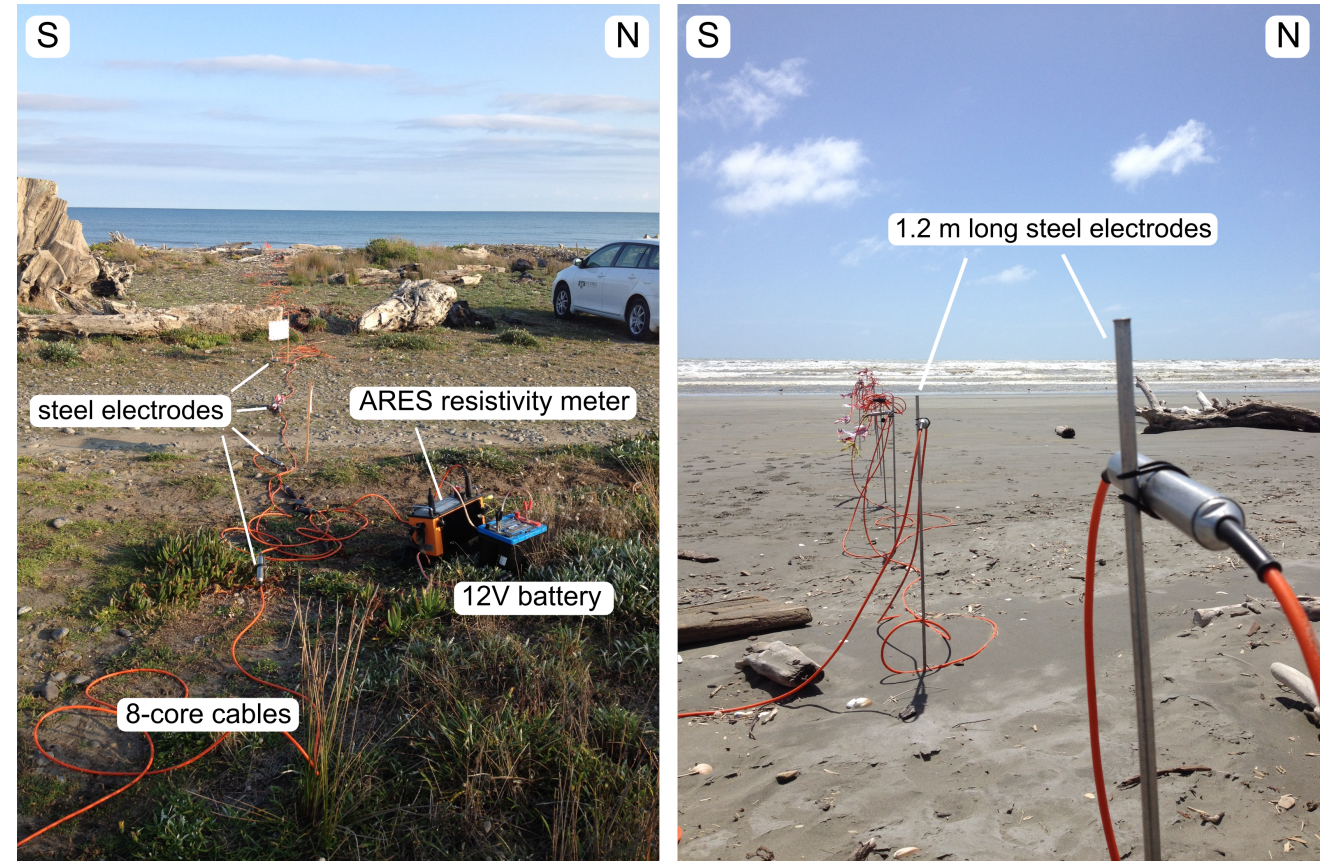

FIGURE 4.3: The resistivity equipment as set up in the field (shown here for a $2-\mathrm{m}$ spaced tidal measurement). On the left side regular $0.35 \mathrm{~m}$ long stainless steel electrodes are connected to the 8-core cable take outs (every $5 \mathrm{~m}$ ) with elastic O-rings. Up to six cables were connected (64 channels) for some of the longest transects. A $12 \mathrm{~V}$ deep cycle battery was used to power the ARES resistivity meter. In the right picture the set up on the $1.2 \mathrm{~m}$ long electrodes is imaged. This was used at the beach to avoid seawater contamination of the equipment there (note, this is shown for a $2 \mathrm{~m}$ minimum spacing survey).

\subsection{Data editing and inversion strategies}

Raw data were edited where necessary for the removal of faulty measurement points. For time-lapse inversions, it is preferable to only compare data points that occur in all time steps (e.g. Johnson et al., 2012; Kobierska et al., 2015). Therefore, missing data points were deleted in all datasets for the same profile. This strict editing approach led to removal of as little as $0.5 \%$ and up to $30 \%$ of the total data points in each data set. The Waikanae profiles N1, N2, N3 and S1 were the ones with the most data points removed. All other transects did not loose more than $12 \%$ of their originally measured data points. Removal of many data points and the associated increase in interpolation during the inversion process is likely to be reflected through the appraisal process. Therefore, it is expected that larger portions of the resistivity models are blanked out for the northern Waikanae profiles than for the rest of the herein discussed data sets. For the Waikanae profiles, it was not possible to run reciprocal measurements for error estimation due to time constraints from tidal and wave action, as well as the use of 
the roll-along technique for the northern three profiles (N1, N2, N3). However, reciprocity measurements have been carried out with the same instrument and electrode geometry and in a similar geological environment on the three profiles $15 \mathrm{~km}$ north in Te Horo. From these measurements, a mean error estimate of $2.5 \%$ (including systematic and random errors) was determined, and this is assumed to be representative of the data sets of the Waikanae study period. In Te Horo, reciprocity measurements were possible for all three transects (TH1, TH2 and TH3) and have therefore been used to provide a direct error estimate there. Details for error estimation procedure can be found in Section 3.2. Total errors for the Te Horo data sets range between $1.55 \%$ and $2.61 \%$. The error estimate for each data point has been included in the time-lapse inversion to give less weight to data with larger errors.

All datasets were inverted in a time-lapse style with the commercially available software Res2DInv (32-bit version) (Geotomo, Malaysia), where, for each time step, the inversion model obtained for the initial time step is used as the reference model in a difference inversion style as suggested by LaBrecque and Yang (2000). A robust model and data constraint $\left(l_{1}\right.$-norm) was used for the least squares inversion (deGroot-Hedlin and Constable, 1990; Sasaki, 1992) to account for the sharp resistivity changes close to the saline interface position. Finite-element modelling and a complete Gauss-Newton solver were regarded appropriate due to the topographical information included and the relatively small $(<3000)$ number of data points collected for each profile. To allow for individual changes of the different time steps in the time-lapse series, rather than achieve similar models by making the difference between models small, a time-lapse damping factor of 0.85 was used together with a smooth change of resistivity between adjacent model blocks (Loke et al., 2006). To allow for the errors in the data, the inversion was stopped once the root mean square (RMS) error was smaller than the estimated total error of the respective data set. All time-lapse inversions converged within three to five iterations to a value below or close to this, and all RMS errors were below those obtained for single inversions of the individual time steps, illustrating the benefit of using a time-lapse approach to reduce image artefacts caused by data noise (Ogilvy et al., 2009).

To only interpret parts of the inversion models that are constrained by the data rather than by the background model, the DOI index method first introduced by Oldenburg and Li (1999) and further developed by Marescot et al. (2003) has been used with the $l_{1}$-norm modification 
introduced by Deceuster et al. (2014). Apart from the Te Horo transects TH1 and TH2, the DOI index data are not bimodally distributed for any of the other profiles. Neither do the DOI index contour lines get closer together, which would theoretically mark the depth of investigation. As a result, based on suggestions from previous DOI index applications (Marescot et al., 2003; Hilbich et al., 2009), the cut-off factor for the DOI index was chosen to be 0.2 for all of these profiles. Two additional time-lapse inversions with 0.1 and 10 times the arithmetic mean resistivity of the original inversion model set as their homogeneous starting model, using an extended model space with five times the pseudo depth of each profile and the same inversion specifications as the original time-lapse inversion, have been performed for each profile and each time step (138 additional inversions for the total of the nine traverses). This way, it could be determined which portions of the models were constrained by data and which were constrained purely by the background model. A mean of the DOI indices of comparing time steps was used to scale the resistivity ratio maps.

\subsection{Monitoring results from two groundwater zones}

In the following the individual results from the two monitoring experiments within the groundwater zones of Waikanae and Te Horo, respectively, are introduced. While the Waikanae experiment can be understood as a pilot study of how the resistivity structure in a shallow coastal sand aquifer changes over the seasons, the measurements at Te Horo Beach serve as a follow up survey with higher time resolution in order to confirm indications from the Waikanae study and outline possible differences. The shift of study location results from the change of direction during mid-project as outlined in Section 1.4. For all models shown in the following images, the opacity of each model cell is scaled using the DOI index so that model cells that are constrained by the field data are fully opaque (a DOI index of 0.0 getting a transparency value of 1.0), whereas those exceeding the cut-off DOI index value were blanked out, and the transparency of model cells with DOI values in between was scaled according to their index value (1.0 - abs(DOI index)). A 45- $\Omega \mathrm{m}$ contour line has been superimposed on the inversion model plots marking the approximate resistivity value that, using the implications from Wilson et al. (2006) and measurements of freshwater conductivity of surface water in the area, was 
considered to represent $1 \%$ of saline mixing. The contour lines on the ratio plots mark zero change in resistivity between time steps.

\subsubsection{Waikanae groundwater zone}

Figure 4.4(a) shows the six models produced by the time-lapse inversion of the DC resistivity measurements collected along profile line N2 (location see Fig. 4.1). These are representative of the results along all six profiles acquired in the Waikanae groundwater zone (Appendix $\mathrm{H}$, Figs. H.1 - H.5). Figure 4.4(b) shows the resistivity ratios between each time step and the previous one for the same profile. The monitoring period for the N2 traverse, at 305 days in total, was the longest, 25 days longer than the shortest monitoring period on traverse S3. This difference results from logistical constraints that did not always allow the repeat data along the different profile lines to be collected in an exact 60-day cycle. A projection of geological well information obtained between profiles S1 and N3 is shown in Fig. 2.5. Based on the geological information presented in Section 2.3.2, following Wilson et al. (2006), who estimated a formation factor of 2.6-2.9 for the Kapiti Coast shallow aquifer, and using typical resistivity values of common earth materials (Palacky, 2006; Reynolds, 2011), low resistivity values $(<1-7 \Omega \mathrm{m}$, blue) in the inversion models are inferred to be associated with saline-watersaturated sands. The transition between saltwater- and freshwater-saturated sands is marked by the resistivity range of about 7-50 $\Omega \mathrm{m}$. Higher resistivities (50-120 $\Omega \mathrm{m}$, green-yellow) indicate freshwater-saturated sandy gravels, whereas values between 120 and $350 \Omega \mathrm{m}$ (yellow-orange) represent freshwater-saturated compacted sands. Shallow very high resistivity (red) areas are interpreted as semi-saturated to unsaturated sands.

The saline interface is thought to be marked by the gradually increasing resistivity contours (from blue to green colours) at the western end of the profiles that represents a mixing zone between saltwater and freshwater rather than a sharp boundary. On none of the profiles does the shape of the saline interface match the classic shape of the saltwater-freshwater transition zone predicted by the Ghyben-Herzberg relationship for which the depth to the saline interface (z) is predicted to be approximately about 40 times the height of the freshwater table above mean sea level (h) (Fig. 1.1). However, it is in agreement with observations made in an earlier saline interface study (Morrow et al., 2010) carried out in the close vicinity of the present 


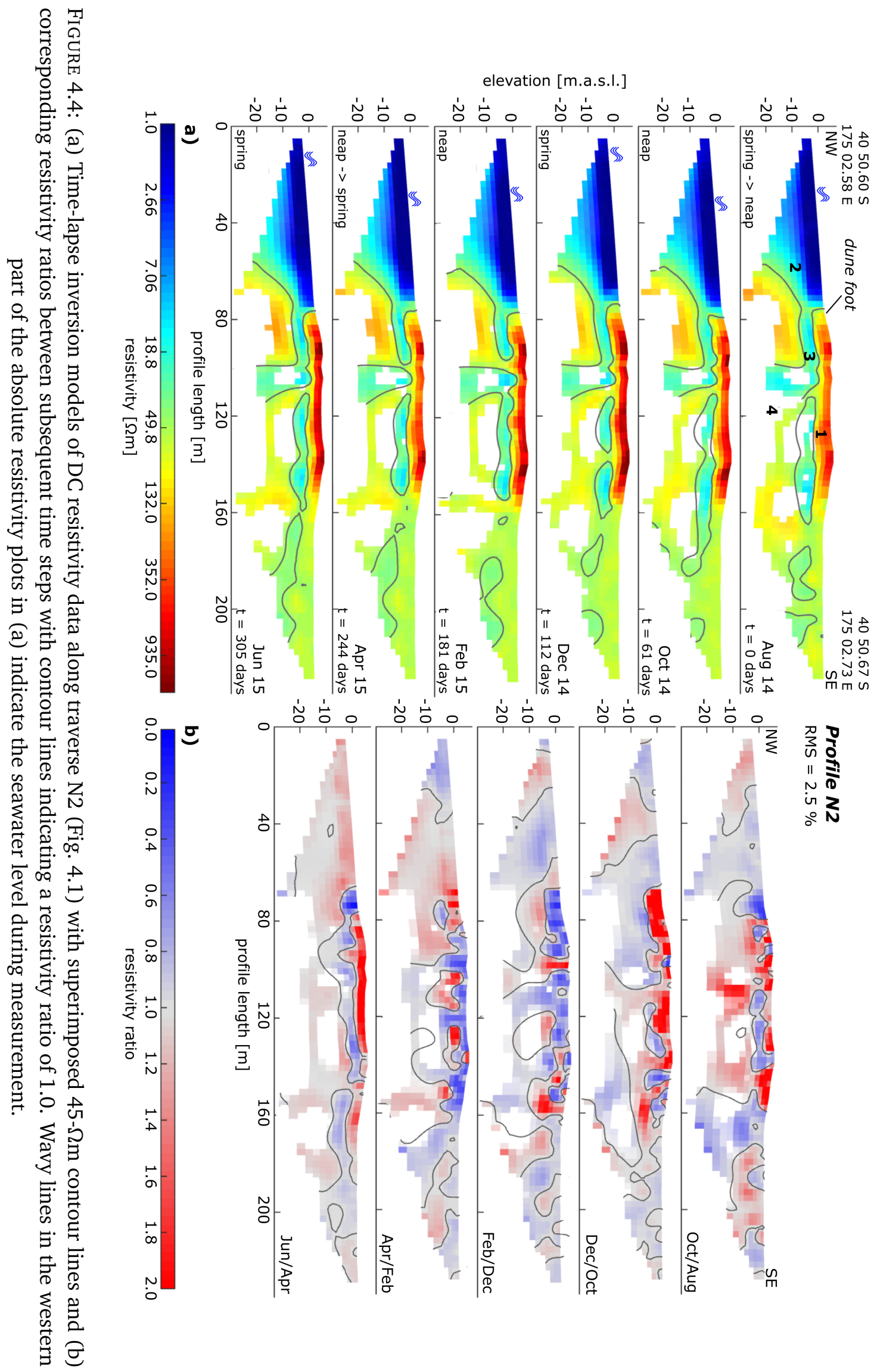


traverses. It is possible that the interface observed on the DC resistivity models may reflect the nearshore circulation zone, rather than the saltwater interface.

Except for profile N1, where it is located $30 \mathrm{~m}$ west of the fore dunes, the saline boundary is located just at the foot of the fore dunes. In general, the beach part of the profiles shows very low resistivity values, whereas in the dune-dominated part of the traverses, the near-surface resistivity significantly increases with distance from the coast. This correlates with the surface saturation found at the measurement locations - on data collection dates, the beach sands were usually saturated, whereas dune sands were normally dry. In profile N2, the eastern end of the profile was located on a wetland area, which, during winter months (August/October 2014 and June 2015), was saturated to the surface. The inversion results in this region (Fig. 4.4(a)) suggest that the water table was close to the surface at all time steps. Underneath the dry surface layer ( $\sim 5 \mathrm{~m}$ thick), a moderately conductive (7-50 $\Omega \mathrm{m}$ ) layer can be seen on all inversion models. This appears to be connected to the saline zone at the seaward end of the profiles and extends a considerable distance inland. Beneath this layer, the resistivity is generally higher, and the structure is both more heterogeneous and differs between traverses. This zone extends to the maximum investigation depth, and the DOI index suggests it to be less well constrained by the data.

In summary, the resistivity structure for all traverses can be basically divided into four main features (as marked for August 2014 in Fig. 4.4(a)):

1. a near-surface layer (approximately the top $5 \mathrm{~m}$ );

2. a saline and mixing zone in the western part of the profiles;

3. a low-resistivity region that is generally at depths between about 5 and 10-15 m below ground level but shows considerable variability in thickness;

4. an underlying higher resistivity region that, on some of the profiles, shows considerable inhomogeneity.

The major changes with time of these four features observed for traverse N2, shown in Fig. 4.4(b), are generally representative of all six traverses although local differences in micro-geology, vegetation, or recharge/discharge processes do lead to slightly different resistivity distributions 
along the various profiles (see Appendix H Figs. H.1 - H.5). To illustrate this similarity in resistivity changes, in Figs. 4.5 and 4.6, the resistivity ratios for all six profiles for February 2015/August 2014 and June 2015/February 2015, respectively, are shown and discussed in the following. The mean changes in the groundwater table stage measured in well R26/6287 (Fig. 4.1) for these two periods amounted to a drop of $355 \mathrm{~mm}$ and a rise of $636 \mathrm{~mm}$, respectively.

For all measurement locations, the near-surface saline transition zone appears to move back and forth by about $\pm 10 \mathrm{~m}$ over the entire monitoring period. For traverse $\mathrm{N} 2$, this is well imaged in the uppermost $5 \mathrm{~m}$ in the resistivity ratio plots (Fig. 4.4(b)) at around a 70- to 80-m profile length, but is also apparent in the resistivity models shown in Fig. 4.4(a). A comparison of the tidal stage at the time of measurement with the saline interface does not show a correlation eastward shifts of the interface are not necessarily connected to higher tidal levels, and vice versa for westward shifts and lower tidal levels (Fig. 4.4(a); October- December and April-June). Neither, within the resolution of the measurements, is there any indication of a correlation of the saline interface location with the overall tidal height (i.e., neap, spring, or in-between). The same observations can be made for the other traverses.

Changes in the near-surface high-resistivity layer (feature 1 in Fig. 4.4) occur at all time steps. For example, on traverse N2 (Fig. 4.4(b)), there is a general increase in the resistivity of this layer between August and December 2014 (first two ratio maps in Fig. 4.4(b). This is followed by a decrease in resistivity in February and April 2015, before a subsequent increase in June 2015. However, from a seasonal perspective (Fig. 4.5), the resistivity of this layer markedly increased between August and February. Thus, although there is clearly a seasonal component to these changes, which is largely reflected in the groundwater level shown in Fig. 2.3(a), an effect due to short-term weather events (Fig. 2.3(b)) is also apparent, with the resistivity of the upper layer being controlled by the shallow subsurface moisture/water content (i.e. vertical shift of the vadose zone (= transition between saturated and unsaturated zone)). This is obviously highly dependent on local rainfall patterns and may change on a year-to-year basis. Based on regular temperature measurements taken in the Te Horo groundwater zone for depths between 4 and $22 \mathrm{~m}$ from the surface, temperature changes with time have been considered negligible with the subsurface temperature variation between seasons being in average in the range of 1 ${ }^{\circ} \mathrm{C}-2{ }^{\circ} \mathrm{C}$ (as discussed later in Chapter 6). 

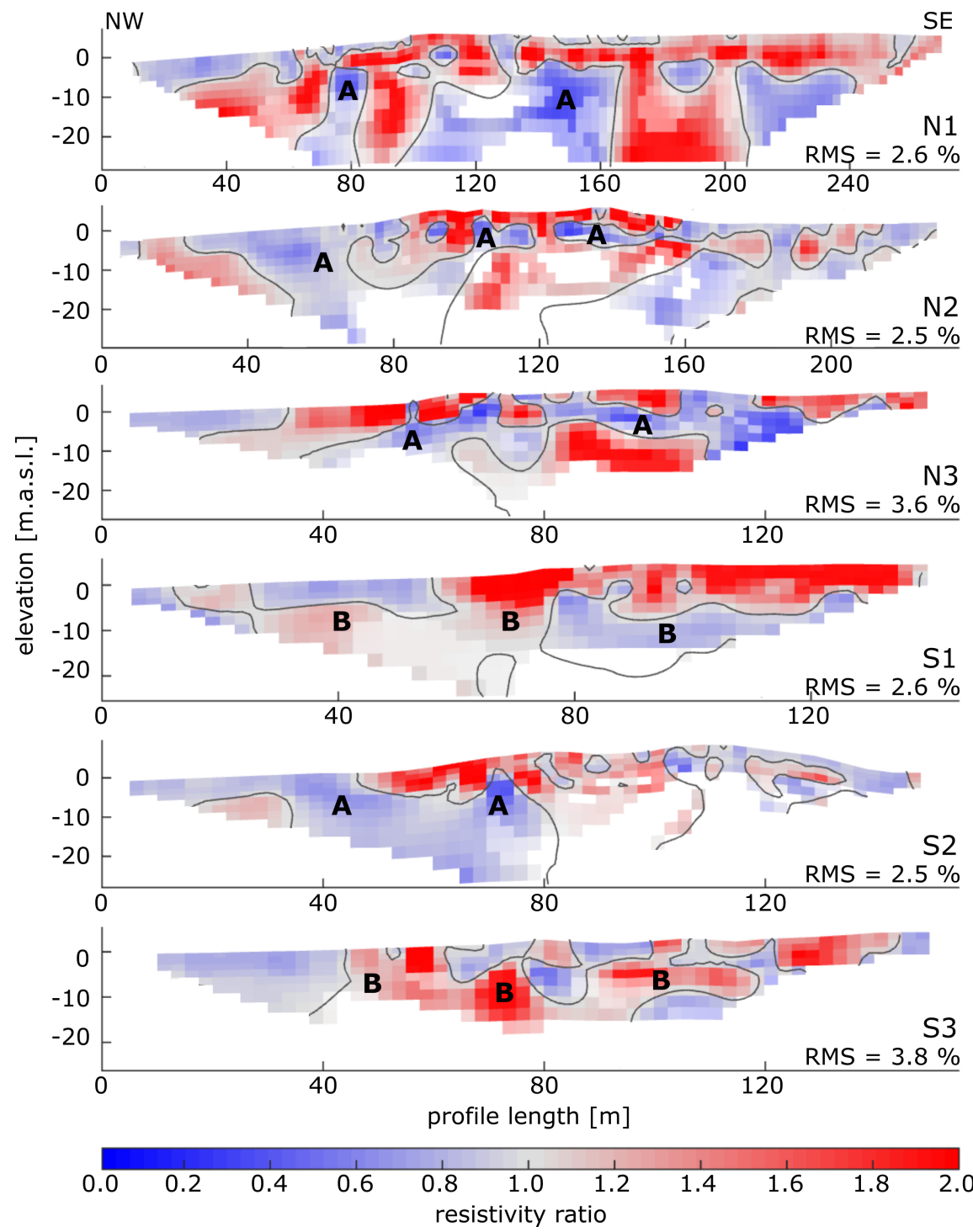

FIGURE 4.5: Resistivity ratios of the DCR time-lapse inversion models for all six profiles (north to south order) between August 2014 and February 2015 ( $\mathrm{R}=$ Feb/Aug). The images were scaled with the DOI index method (opacity level), and the contour lines mark a resistivity ratio of 1.0 (no change) between the two time steps investigated. Water levels dropped in average by $355 \mathrm{~mm}$ in well R26/6287 (see Fig. 4.1) between the August and February acquisition dates of the profiles.

Below about $5 \mathrm{~m}$ depth (features 3 and 4 Fig. 4.4(a)), there are somewhat systematic changes in resistivity with time. Between August 2014 and February 2015, there are significant regions where the resistivity decreases with time. These are marked $A$ in Fig. 4.5 for traverse N2 and include the thin layer of lower resistivity that extends across the traverse at a depth of about $5 \mathrm{~m}$. Generally, to the northwest, these regions appear to be connected to the saline boundary zone. Similar regions in which the resistivity decreases from August to February are also apparent on traverses N1, N3, and S2 (Fig. 4.5). From February 2015 to June 2015, there is then a subsequent increase in resistivity in these regions (again, marked $A$ in Fig. 4.6) with similar 
increases occurring on N1 and to a lesser extent on N3. However, on traverse S2 over this period, the resistivity continues to decrease. This may well reflect a more general difference between the more rural traverses (N1, N2, and N3) and the more urban traverses (S1, S2, and S3) as on S1 and S3, the pattern of resistivity changes seen on N1, N2, and N3 is reversed with an increase in resistivity below about 5 m depth between August 2014 and February 2015 being followed by a decrease (regions marked $B$ in Figs. 4.5 and 4.6).
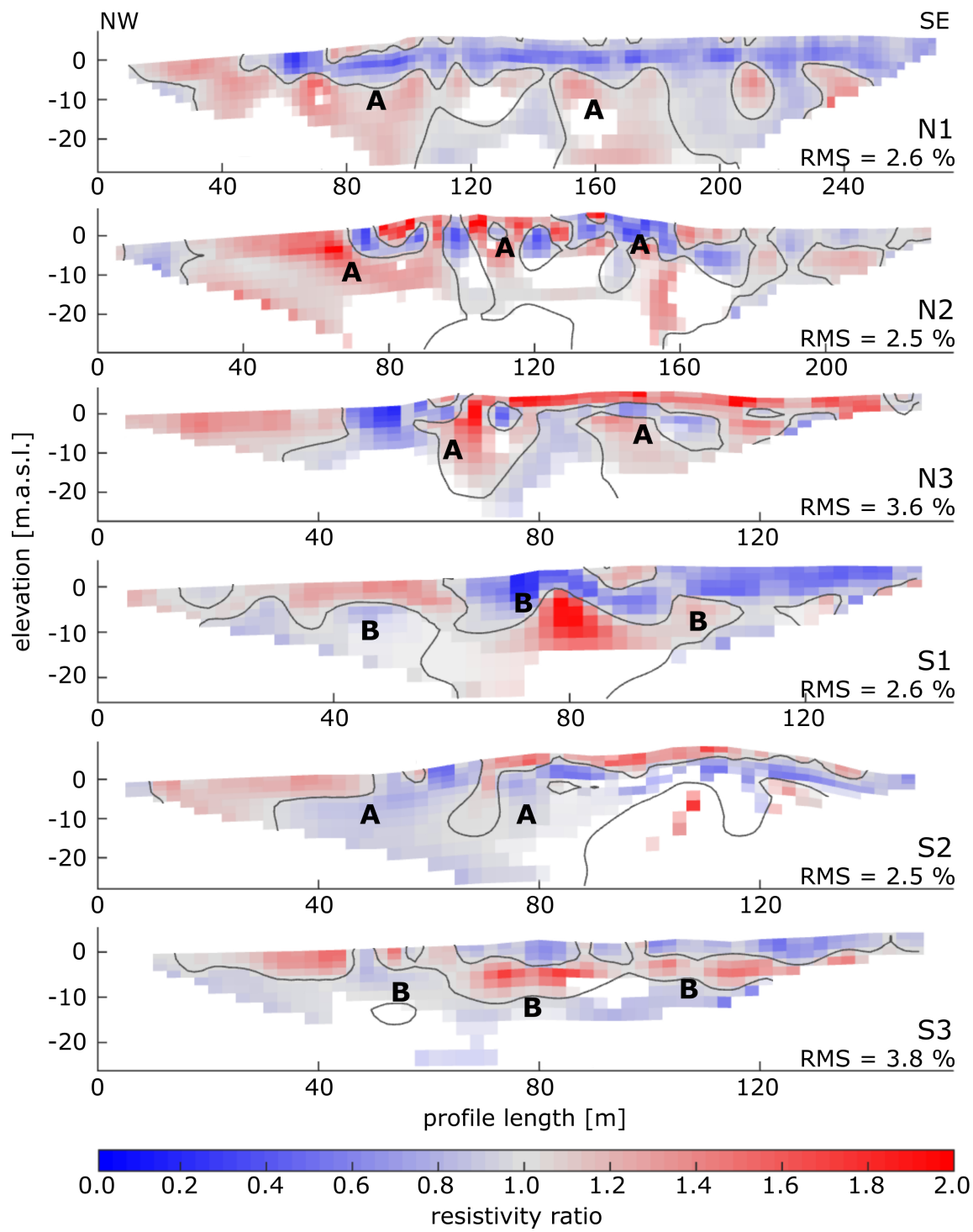

FIGURE 4.6: Resistivity ratios of the DCR time-lapse inversion models for all six profiles (north to south order) between February 2015 and June 2015 ( $R=$ Jun/Feb). The images were scaled with the DOI index method (opacity level), and the contour lines mark a resistivity ratio of 1.0 (no change) between the two time steps investigated. Water levels increased in average by 636 $\mathrm{mm}$ in well R26/6287 (see Fig. 4.1) between the February and June acquisition dates of the profiles. 
Depths below about $15 \mathrm{~m}$ are not well resolved for the profiles N3 and S1-S3, and only the traverses N1 and N2 yield some limited information at these depths. From August 2014 to February 2015 (Figs. 4.4(b) and 4.5), there is a similar decrease in resistivity at the western end of the profiles (particularly N2) to that discussed above. Again, this change reverses (Figs. 4.4(b) and 4.6) with more freshwater present in the aquifer from February to June. However, the resistivity increase in this layer coming into winter is about $50 \%$ less than the decrease from August to February.

\subsubsection{Te Horo groundwater zone}

Similar to Fig. 4.4 for the Waikanae groundwater zone, Fig. 4.7(a) illustrates the resistivity models sequence of the seasonal Te Horo DC resistivity monitoring along profile line TH1 (location see Fig. 4.2). As the three transects monitored at this location are only about $200 \mathrm{~m}$ apart from each other and results do not differ markedly along these, the middle profile TH1 will serve as a representative for monitoring results discussion in the following (results for TH2 and TH3 see Appendix H Figs. H.6 and H.7). The sampling interval was halved to represent a roughly 30-day interval as compared to the pilot study in Waikanae. From the resistivity models it appears that the same four features outlined in Section 4.4.1 can be identified at the Te Horo field site, which makes sense considering the similarity in geologic deposition history. The only striking difference is the Te Horo Gravel Beach, a thin layer $(\sim 0.5 \mathrm{~m})$ of coarse well-rounded gravel on top of the dune sands between about 20 and $70 \mathrm{~m}$ profile line extension on TH1. Though it may influence local rainfall infiltration at this location, this feature is most likely not resolved in the 5-m minimum spaced survey due to its small vertical extent. Therefore, the resistive top layer in Fig. 4.7(a) is thought to represent again semi-saturated to dry sand (feature 1) that is mainly controlled by groundwater level and moisture content related to short term precipitation or evapotranspiration conditions.

In the north-western part of the profile within the first $50 \mathrm{~m}$ a saline and mixing zone is again apparent and characterised by low resistivity values $(<1-50 \Omega \mathrm{m})$. The saltwater saturated sands seem to be located further away from the dune foot here compared to the Waikanae profiles and therefore this zone makes up a smaller percentage of the imaged ground in the northwest of the profiles. The smaller portion of saltwater saturated sands in Te Horo might 
be the reason for the slightly differing shape of the transition zone. Nevertheless, it is also far from representing a classical Ghyben-Herzberg type of interface. In order to get a clearer image of the saline zone at the northwestern end of the profiles, a pseudo-3D geoelectrical survey was carried out, which is discussed in Appendix A. This suggests that the saline wedge is a real feature rather than a product of inversion artefacts, showing an N-S continuous layer of $\sim 10$ $\mathrm{m}$ thickness along the coast and overlying higher resistive parts of the aquifer. The so-called unstable situation may be a product of a mildly sloping beach, discharge of freshwater upwards at the beach and / or sea storm events. As was observed on the resistivity models from the Waikanae monitoring series, the horizontal position of the saltwater interface is not related to either tidal wave run-up during the measurements, nor to spring-neap tide cycles. In contrast to Waikanae observations of the interface position with time it appears that in Te Horo the horizontal movement of this feature is, though within approximately the same fluctuations of 10 $\mathrm{m}$, continuously shifting towards the coast when coming from winter into summer and back for the reverse situation. This is also apparent on the resistivity ratio plots in Fig. 4.7(b). The three last time steps from June to August 2016 mark special cases where due to two severe sea storms in late May and late July 2016 wave run-up much further inland than normal was observed. These events had a clear influence on short-time resistivity distribution and overprinted seasonal patterns as can be seen in Fig. 4.7 on the last three inversion models and model ratios (Jun/May, Jul/Jun and Aug/Jul). It is also noteworthy that the February 2016 monitoring was carried out twice - once during the lowest groundwater stage in the shallow aquifer (Feb1) and then again just after a heavy rain event (Feb2) that raised the groundwater table by approximately 250 $\mathrm{mm}$ for a short time (Fig. 2.7(a)).

Towards the south-east of the profile a 5-10 m thick less resistive layer (feature 3) below the dry surface sands appears to be connected to the transition zone. This approximately matches with an increased amount of gravel in the sand at this depth interval as inferred from bore logs of the area (Fig. 2.8). At depths below about $10 \mathrm{~m}$ the resistivity again increases to roughly between 120 and $350 \Omega \mathrm{m}$ (yellow-orange) representing the freshwater-saturated compacted sands referred to as feature 4 in the Waikanae groundwater zone resistivity models. The Te Horo model appraisal for TH1 was done using the statistically obtained DOI cut-off factor as introduced by Deceuster et al. (2014) instead of taking a fixed value of 0.2 as for the Waikanae monitoring. This may have led to a less restrictive (though, possibly more accurate) estimation 


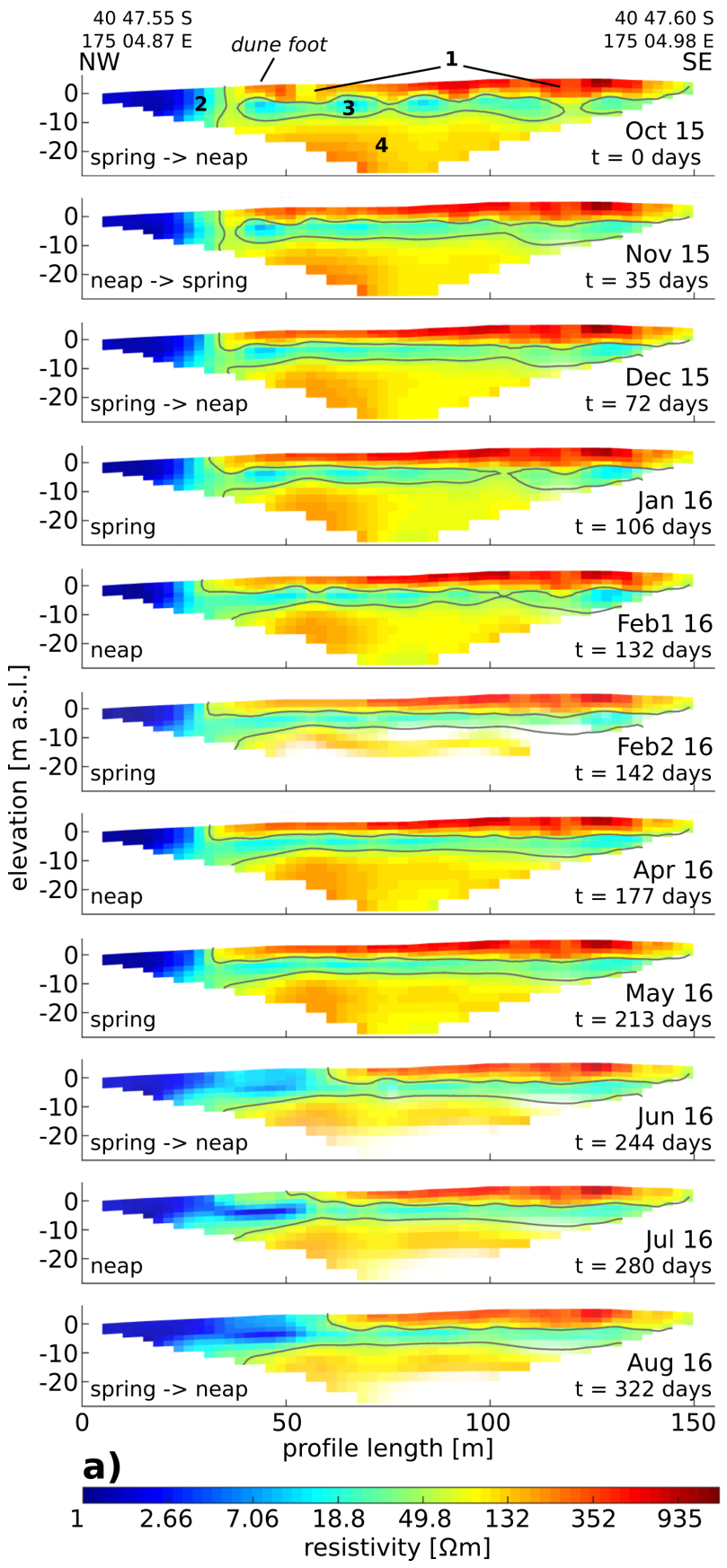

\section{Profile TH1 RMS $=2.7 \%$}
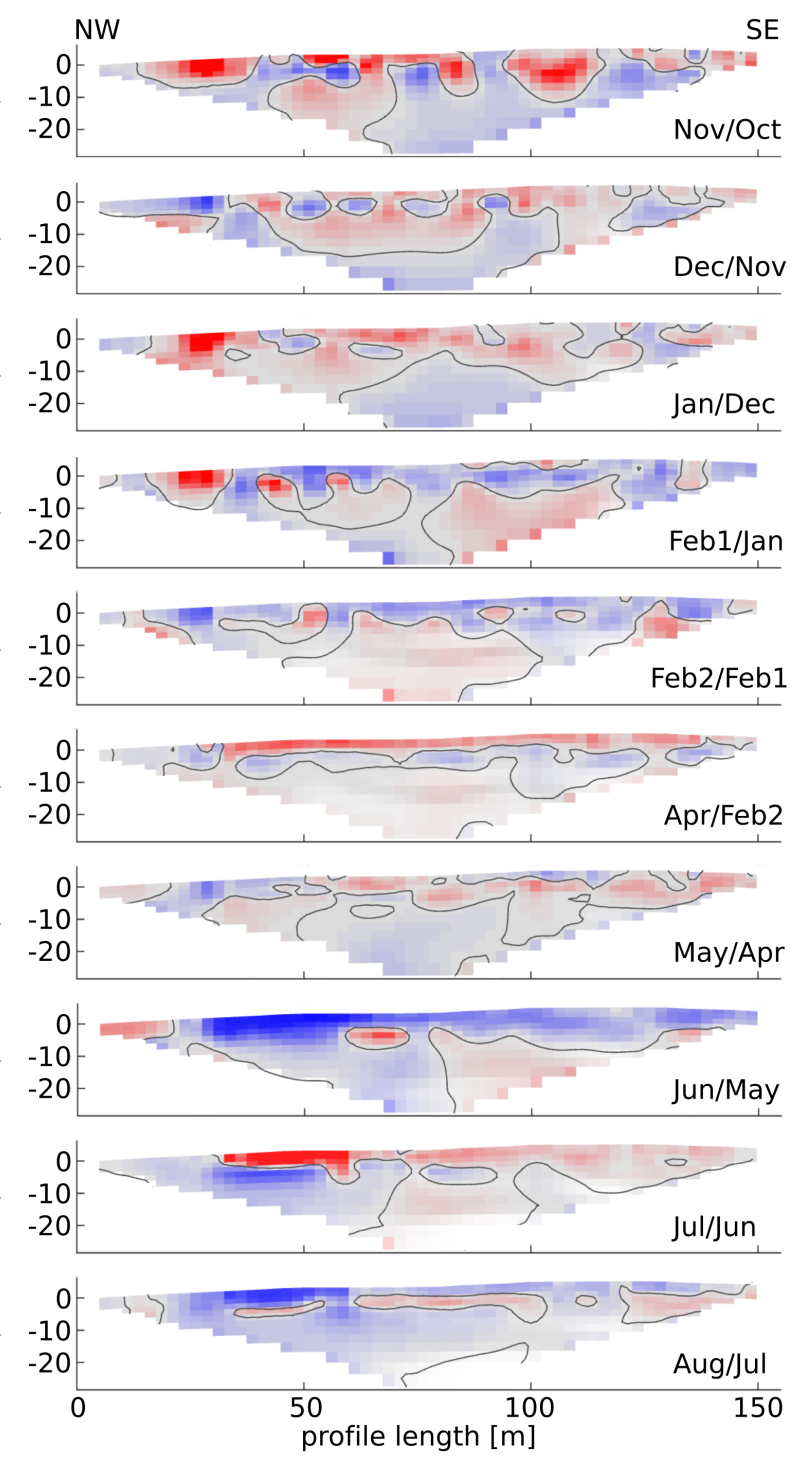

\section{b)}

FIGURE 4.7: (a) Time-lapse inversion models of DC resistivity data along traverse TH1 (Fig. 4.2) with superimposed $45-\Omega \mathrm{m}$ contour lines and (b) corresponding resistivity ratios between subsequent time steps with contour lines indicating a resistivity ratio of 1.0. The wave run-up during measurements only reached the electrodes in the January and August 2016 surveys (up to the second electrode at $5 \mathrm{~m}$ profile extent). 
of the reliability of the inversion models in Fig. 4.7 at depth.

Changes in the resistivity distribution with time can be observed for subsequent time steps along profile TH1 in Fig. 4.7(b). There, the ratio of the resistivity of each model cell between adjacent monitoring times illustrates large heterogeneity in the uppermost surface layer which was already earlier accounted to local precipitation and evapotranspiration conditions and does not seem to exhibit a distinct seasonal component. In terms of seasonal saltwater intrusion patterns, it is much more interesting to investigate the behaviour of the conductive middle layer (feature 3) with time. Although, this is demonstrated to a certain extent from the step wise resistivity ratios in Fig. 4.7(b), it is much more pronounced for resistivity ratios over a seasonal time frame. In Fig. 4.8 the ratios between the October 2015 and February 2016 models are shown for all three profiles measured in Te Horo. This illustrates the change of resistivity coming from winter into summer with a drawdown of the groundwater table height by roughly $400 \mathrm{~mm}$ during this period (see Fig. 2.7(a)). In order to avoid misinterpretation from the heavy rainfall in mid-February 2016 the data set that was acquired prior to this event (Feb1) was used for comparison. It marks one of the lowest groundwater table stages measured during the entire monitoring period. On all three profiles a clear increase in resistivity is observed in this more conductive layer between about 5 and $15 \mathrm{~m}$ depth. Because an increase in resistivity from winter into summer was marked with $B$ on the Waikanae profiles in Figs. 4.5 and 4.6, this nomenclature is continued here. In contrast, the lowest part of the aquifer indicates a decrease in resistivity with the decrease in groundwater stage.

The opposite can be observed in Fig. 4.9 where the ratios for May 2016/February 2016 for the three Te Horo profiles are illustrated. There, the aquifer parts that were marked with $B$ in Fig. 4.8 to identify regions with increasing resistivity, show that a decrease in resistivity with increasing groundwater stage is dominant for the middle layer. This is accompanied by a resistivity increase in the lowest parts of the profile section. Again, resistivity changes from winter into summer (Fig. 4.8) are more pronounced (up to about $50 \%$ ) than is the case for those from summer into winter (Fig. 4.9). With this behaviour, the Te Horo field sites demonstrate similarity to the urban profiles S1 and S3 in Waikanae.

Figure 4.10 illustrates the effect of exceptional events on the resistivity ratio comparison. Instead 


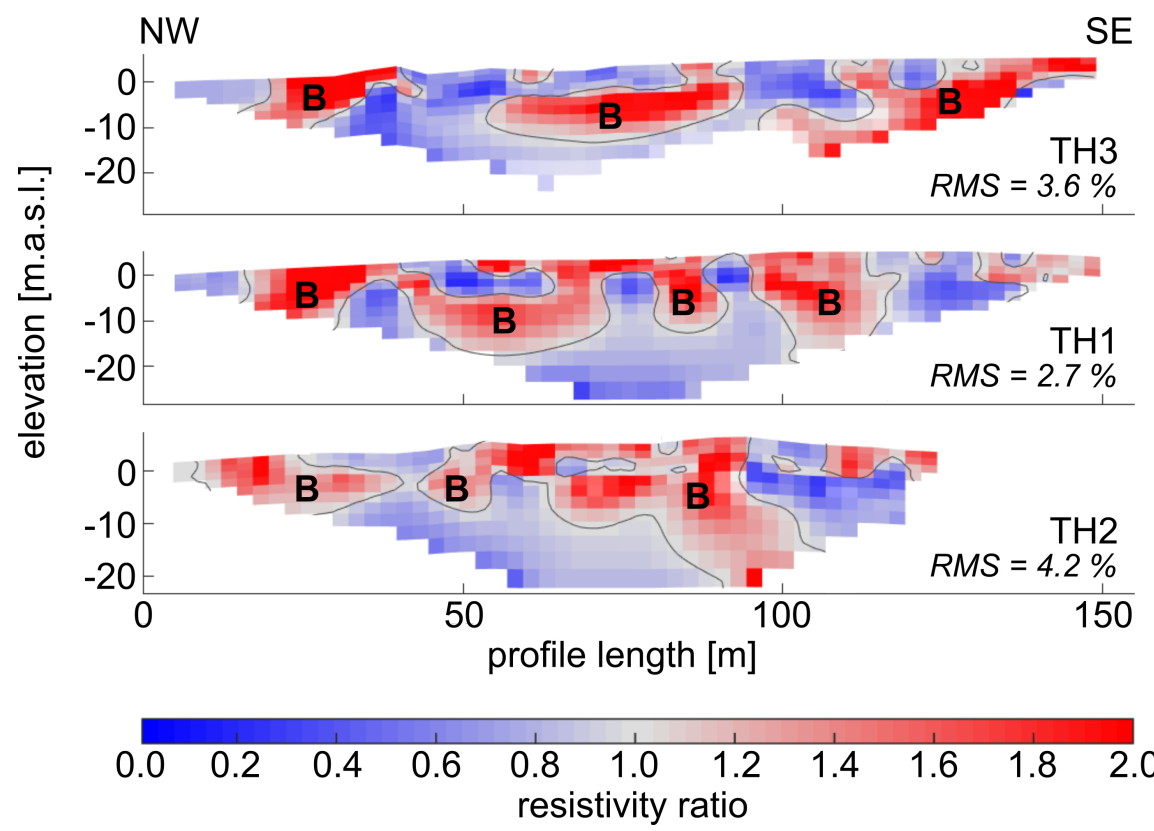

FIGURE 4.8: Resistivity ratios of the DCR time-lapse inversion models for all three profiles (north to south order) between October 2015 and February $2016(\mathrm{R}=$ Feb/Oct), where the February data set taken for the ratio calculation was the one previous to the rain event. Water levels decreased in average by $395 \mathrm{~mm}$ at the Te Hapua wetland monitoring site (GWRC monitoring network (location see Fig. 2.1)) between the October and February acquisition dates of the profiles. The letter $B$ marks the increase in resistivity of the conductive middle layer.

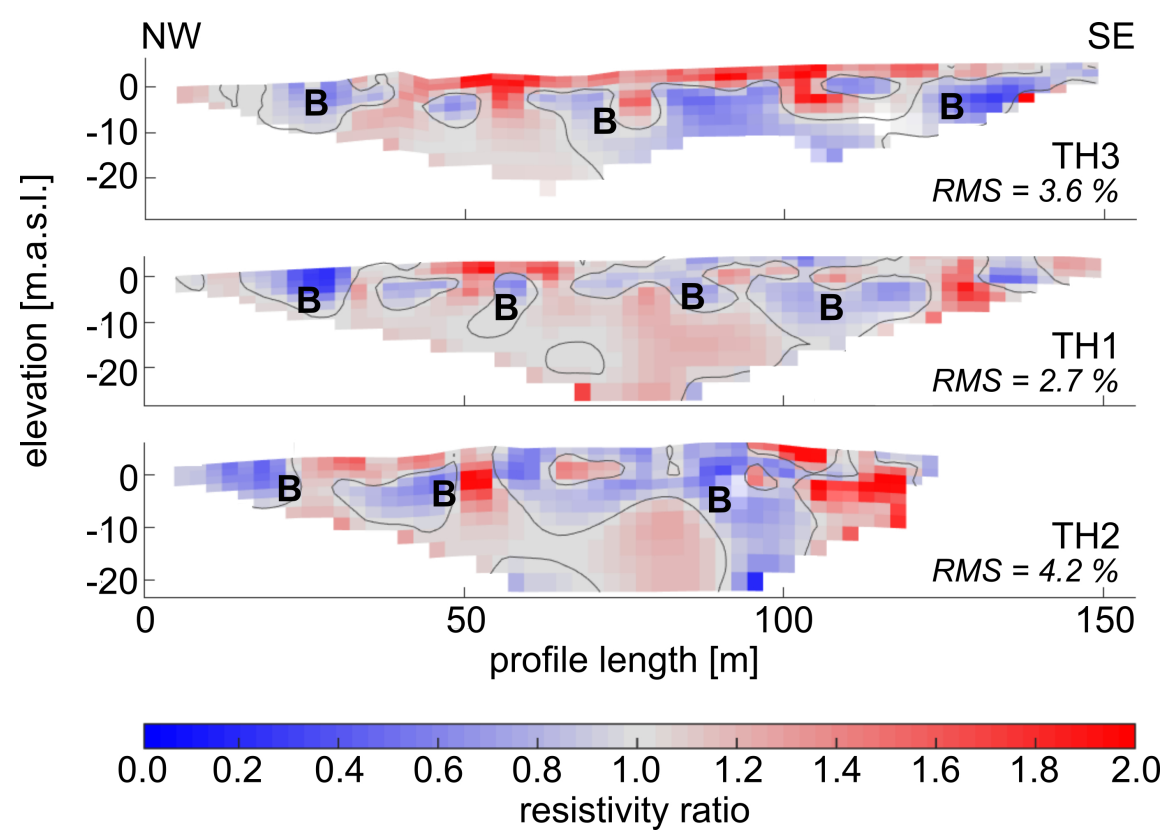

FIGURE 4.9: Resistivity ratios of the DCR time-lapse inversion models for all three profiles (north to south order) between February 2016 and May 2016 (R = May/Feb), where the February data set taken for the ratio calculation was the one previous to the rain event. Water levels increased in average by $124 \mathrm{~mm}$ at the Te Hapua wetland monitoring site (see Fig. 2.1) between the February and May acquisition dates of the profiles. The letter $B$ marks the decrease in resistivity of the conductive middle layer. 
of taking the ratio May 2016/February 2016 as was done for interpretation in Fig. 4.9, the seasonally more intuitive time steps of February 2016 and August 2016 were compared. It appears that large parts of the shallow aquifer experienced a substantial (40-100\%) decrease in resistivity between the lowest and the highest groundwater stage (increase of $437 \mathrm{~mm}$ from February to August 2016). And although some features experience similar behaviour as for the ratios May/Feb (see parts marked with $B$ ), especially the observations at the north-western part of the profiles are masked by the consequences of the two severe sea storms mid-year 2016. Due to flooding of the beach face up to a profile length of $70 \mathrm{~m}$ with seawater during these two events it is suspected that the saline water was seeping into the shallow aquifer from the surface, thus significantly lowering the subsurface resistivity as is also indicated in Fig. 4.7 (Jun-Aug).

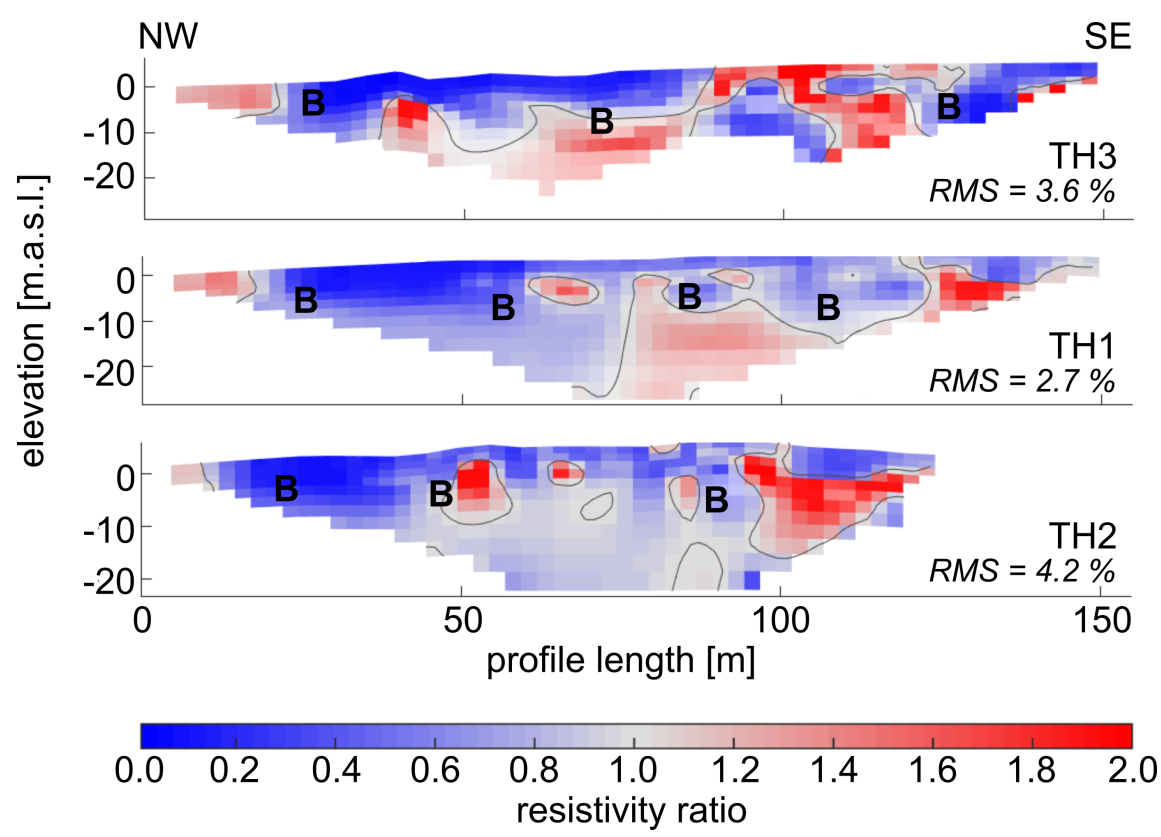

FIGURE 4.10: Resistivity ratios of the DCR time-lapse inversion models for all three profiles (north to south order) between February 2016 and August 2016 (R = Aug/Feb), where the February data set taken for the ratio calculation was the one previous to the rain event. Water levels increased in average by $437 \mathrm{~mm}$ at the Te Hapua wetland monitoring site (see Fig. 2.1) between the February and August acquisition dates of the profiles. The letter $B$ marks the decrease in resistivity of the conductive middle layer. 


\subsection{Discussion of seasonal seawater intrusion effects on a shallow coastal aquifer}

One of the objectives of this project is to image the saline interface by means of DC resistivity traversing and to monitor changes in the aquifer resistivity distribution over a seasonal time frame. As already shown in previous studies (e.g. Wilson et al., 2006; De Franco et al., 2009; Ogilvy et al., 2009; Morrow et al., 2010), the geoelectrical method is well capable of detecting the freshwater-saltwater interface position in a coastal aquifer. Moreover, resistivity changes within a seasonal time frame can be characterised as demonstrated by the two seasonal monitoring studies carried out during this project.

Morrow et al. (2010) observed resistivity changes associated with changing tidal conditions. To image a seasonal change in the subsurface resistivity distribution rather than a momentary tidal overprint, measurements have been carefully timed and kept within a minimum range of tidal wave run-up. As is demonstrated by the time-lapse inversion models obtained for Waikanae profile N2 in Fig. 4.4(a) and Te Horo profile TH1 in Fig. 4.7(a), the tidal stages (wave run-up) during the measurements do not correlate (i.e. compare October-December with April-June in Fig. 4.4(a)) either with the saltwater interface position or with high/low-resistivity changes as might be expected from a lower/higher wave run-up as suggested by other authors (AtaieAshtiani et al., 1999; Turner and Acworth, 2004; Osborne, 2006). Neither does the time of the measurements within the lunar cycle correlate with a low- or high-resistivity distribution within the aquifer due to a change in the tidal head (spring and neap tides - e.g. October-December in Fig. 4.4(a) or February2-April in Fig. 4.7(a)). There is also no discernible combined effect from wave run-up and tidal head, which could have a reinforcing or cancelling effect on the aquifer state depending on their combination (e.g. February-June in Fig. 4.4(a)). Thus, neither the momentary seawater stage nor the time within the lunar tidal cycle has a resolvable effect on the saltwater interface position, mixing zone dimensions, or resistivity distribution within the aquifer at the chosen Wenner- $\alpha$ array with 5-m minimum electrode spacing.

Resistivity changes in the middle layer (about 5- to 15-m depth - feature 3) of the Waikanae transects indicate that there is a general decrease in resistivity when it can be assumed that 
there is less freshwater present in the aquifer during summer time (e.g. due to lowered recharge and/or enhanced groundwater abstraction) and an increase in resistivity with increasing freshwater pressure in winter. With less freshwater flowing towards the coast the seawater at the saline interface is able to intrude inland. Hence, enhanced mixing of ion-rich seawater with freshwater will decrease the bulk resistivity of the aquifer. Once rainfall is increased towards winter time, more freshwater will flow again through the aquifer, thereby dilute the pore water and as a result lead to higher observed resistivity values in the geoelectrical models. However, there are exceptions to the above observation of resistivity change with time, especially for the more urban southern profiles in Waikanae where this pattern of changes is either not as pronounced (profile S2) or even appears to be reversed (profiles S1 and S3). The reversed resistivity behaviour (increased resistivity with lesser freshwater in the aquifer and vice versa) observed on the urban profiles in Waikanae is very pronounced on all three transects monitored in Te Horo. Better data quality in Te Horo also allowed the aquifer at this location to be better imaged at depth and led to more reliable information about the lower lying part of the aquifer earlier referred to as feature 4. There, the opposite temporal behaviour of feature 3 is indicated. As there is no indication of changes in the inter-annual mean sea level stage (Bell et al., 2000) it is assumed that seawater pressure is not the controlling process in this case. The observations in the urban areas may not follow the expected temporal resistivity behaviour as introduced in Fig. 1.2, but may be explained with additional processes being abundant in the coastal aquifer as the sketched hypothesis in Fig. 4.11 illustrates.

In summer, less freshwater recharge leads to a drop in the water table height in features 1 and 3. Enhanced evapotranspiration resulting from higher received energy, temperatures and associated increases in humidity deficits along with increased surface irrigation additionally dries the now semi-saturated layer and salt crystallizes in the pore matrix. This process decreases the total dissolved solids content of the remaining water in feature 3 and therefore leads to a higher observed resistivity in the geoelectrical model. Additionally, freshwater flows upwards from feature 4 because the hydraulic head in feature 3 decreased. This leads to an increased freshwater flow towards the coast in feature 3 and also adds to an observed increase in resistivity in this layer. Due to the lowered freshwater pressure the same processes as previously mentioned for the rural profiles in Waikanae are active at the saline interface. More seawater can intrude the aquifer, but because there is a higher flow of freshwater at the shallower depths (feature 


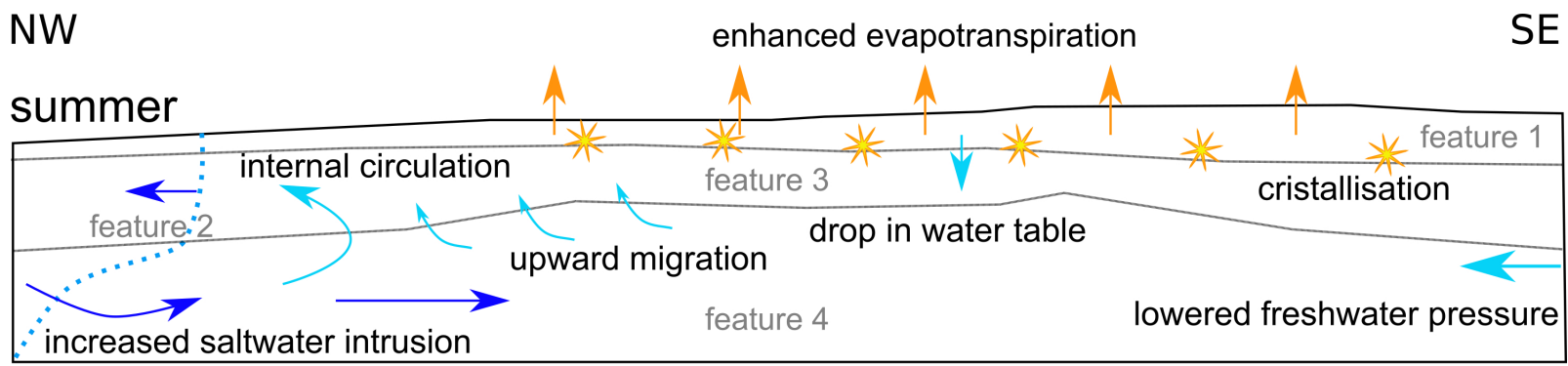

decreased evapotranspiration

winter

\begin{tabular}{lll}
\hline internal, circulation \\
\hdashline decreased saltwater intrusion
\end{tabular}

FIGURE 4.11: Sample aquifer model to illustrate how different processes can lead to unexpected behavior in seasonal time-lapse geoelectrical models as observed along urbanely located profiles in this study.

3) due to a higher hydraulic conductivity in gravel and increased upward migration of water from feature 4 this is more pronounced at larger depths and lowers the observed bulk resistivity only in feature 4. Internal circulation in the transition zone might additionally lead to freshwater being pushed upwards leading to a shift of the saline interface towards the sea in feature 3 .

Later in winter when rainfall is increased and evaporative demand is lower, the aquifer gets recharged with freshwater which leads to an overall rise in groundwater stage. Evapotranspiration significantly decreases and the previously crystallized ions get dissolved again. In the geoelectrical measurements this is then seen as a decrease in resistivity in feature 3. With more freshwater pressure towards the interface less seawater can intrude and the pore water in feature 4 gets more resistive due to dilution through the less ion-rich freshwater. Upward migration reverses to downward migration as the hydraulic head in feature 3 is now larger than the one in feature 4 because of the higher hydraulic conductivity in this layer and hence, its higher freshwater flow. Therefore, internal circulation may be reversed in the mixing zone at the coast, which leads to increased saline mixing in feature 3 and the movement of the saline interface further landward.

The remaining question is to why two different behaviors are observed within a relatively small area. It may be a result of higher compaction within the townships (higher load on soil 
and sediments) and the presence of artificial drainage leading to changed recharge/discharge patterns within the more developed areas, while in rural parts of the aquifer these patterns are mostly undisturbed. As a result, there may be more stagnant water in the aquifer under residential areas during summer when the freshwater recharge is decreased, whereas in the natural sections there might be more groundwater movement because the water can flow in larger circulation cells.

On the Waikanae ratio plots it appears that almost doubling the amount of freshwater in the aquifer (from February to June 2015) has a much smaller effect on the resistivity change on all six profiles at depths larger than $5 \mathrm{~m}$ below surface (compare the rate of change in Figs. 4.5 and 4.6). A similar observation is made for the Te Horo data sets where the change in resistivity is up to $50 \%$ larger for ratios illustrating changes from winter into summer (Fig. 4.8) compared to those between February and May 2016 (Fig. 4.9). However, the difference in groundwater stage for the latter case is also much smaller than between October 2015 and February 2016 in the Te Horo groundwater zone. And while the rate of change in the resistivity ratios is roughly similar for model comparison summer-winter in Fig. 4.10 with similar groundwater stage changes as for the winter-summer period during the monitoring study, this also illustrates a special case and cannot be compared directly. Therefore, while the smaller rate of change from summer into winter in the Waikanae case may indicate a slower process for saline water replacement in the aquifer on a seasonal time scale, this cannot be confirmed by the Te Horo data set.

Along all six Waikanae transects (but only shown in Fig. 4.4(a) between about 60 and 90 $\mathrm{m}$ on the traverse), a common feature is that saline water (low resistivity) appears to overlie a region containing much fresher water (higher resistivity) defining both the shape of the interface and the tongue of lower resistivity at around 5- to 10-m depth. It could be interpreted to be a result of leakage from the underlying semi-confined aquifer unit applying hydraulic pressure from below. This was originally inferred from a regional hydrogeological study carried out by URS (2003) which, from well head comparisons, suggested an upward freshwater flow close to the coast and a downward flow further inland. Gyopari et al. (2014) confirmed this behaviour from observations of river stage measurements on the Waikanae River and Waimeha Stream. At the Te Horo field site the extent of the transects on the seaward side was not sufficient to 
image the saline interface down to a satisfactory depth. Hence, the above interpretations could not be applied to these resistivity models. The lower-resistive slabs at the north-western end of profile TH1 observed in the June to August 2016 models in Fig. 4.7(a) are interpreted as the result of surface seepage of seawater due to flooding during two violent storms mid-2016 and do not account for leakage from a lower lying aquifer unit.

Inland of the mixing zone, the geology (suggested by bore logs obtained near the coast (i.e. R26/6676 in Fig. 4.1)) indicates that a sandy gravel layer at depths of around 5-15 m lies above a fine-grained sand unit containing silt and clay components (Fig. 2.5). Higher hydraulic conductivity in this layer compared with the deeper unit may mean that horizontal water flow is more within the gravel layer and that, although horizontal mixing of saline water occurs in this layer, vertical mixing of seawater only occurs at a minor rate. This is something that is also strongly suggested from saline mixing front velocity calculations done in the two groundwater zones as presented in Appendix B. Nevertheless, as the coastline is prograding (de Lange, 2014), some areas of shallower low-resistivity well inland may reflect relict concentration of saline water. This is possibly more important for the longer transects collected in the Waikanae groundwater zone (N1 and N2).

As noted in Section 4.4.1, for all profiles monitored in the Waikanae area, except profile $\mathrm{N} 1$, the saline interface position is consistently at the foot of the fore-dunes. On N1, its location is $30 \mathrm{~m}$ further west. Although transects N2 and N3 are placed in a similarly rural area, they are much closer to the more developed township than profile N1. Similar observations of differences in the location of the saline interface were made by Wilson et al. (2006) in the Te Horo Beach area more than ten years prior to the current project, where they found an approximately 10-m inland shift of the saline interface position for profile TH1 compared with two rural profiles to the north. Because the exact position of the northern two profiles could not be evaluated, this study does not contain a comparative data set in order to confirm this observation. However, the similar observation of this phenomena at the Waikanae field site suggests a strong correlation between generally enhanced shallow groundwater abstraction and saline intrusion. This is further investigated in Section 6.7.

To supply the community with sufficient freshwater during drier summer months, the Kapiti 
Coast District Council (KCDC) has recently installed an extensive deep bore field in the Waikanae area. Several of these bores are approximately $1.5 \mathrm{~km}$ directly inland of profile lines N2, N3, and S1. Records of pumping activity during the DC resistivity monitoring period show that the bore pumps were extracting groundwater in December 2014 and February and April 2015. Pumping rates were highest in April and amounted to a maximum of $65 \mathrm{l} / \mathrm{s}$ (approximately 40 $\%$ more than for the other months). Given the proposed upward flow from lower aquifer units into the shallow Holocene sand (Q1) aquifer at the coast, a decreased amount of groundwater in the lower units caused by active groundwater extraction could possibly result in lower pressure upward towards the shallow aquifer unit. This could lead to enhanced saltwater intrusion into the shallow aquifer. However, other than the observed decrease in the resistivity below 5- to 15-m depth from winter into summer, there are no significant or related changes, such as a change in the mixing zone thickness or the saltwater interface position, observed on the likely affected profiles. From this, it is inferred that, at least at the current rates, pumping of groundwater from deeper units has only a small influence on vertical groundwater migration from these aquifers and, hence, the amount of freshwater present in the Q1 unit. This is in agreement with both the much smaller drawdown predicted by Gyopari et al. (2014) for the Holocene sand aquifer compared with the deeper aquifer units (Q2-Q6) from modelling extensive pumping of the bore field as well as the hypothesised minor importance of upward flow to saline intrusion at the coast in comparison to horizontal groundwater movement due to a hydraulically more conductive shallow sandy gravel layer at depths of around 5-15 m.

\subsection{Summary}

The results discussed above represent the combined findings from a pilot study in the Waikanae groundwater zone and a follow-up study in the Te Horo groundwater zone further north. They were conducted to investigate suspected seasonal salinity changes in the near-coast portions of an unconfined sand and gravel aquifer. The time scale of the pilot study was chosen to be fairly broad (2-monthly repetition interval) in order to get a first understanding of the complicated dynamics of a coastal shallow aquifer system. Time-lapse inversions for three different DC resistivity profiles within this groundwater zone indicate that resistivity changes with time in rural areas follow the expectations for a rapidly responding shallow aquifer system during a 
seasonal cycle (lower resistivity observed in dry summer months and increased bulk resistivity values during wet winter months). However, three profiles within more urban locations show the opposite behaviour of the aquifer response to changes in the freshwater pressure regime. This is confirmed by a temporally higher resolution follow-up study in the adjacent Te Horo groundwater zone that distinctly shows this behaviour along three transects within the township boundaries. The different seasonal behaviour of the same aquifer in rural and urban areas is thought to be a result of additional processes such as enhanced evapotranspiration / evaporation from irrigation and pumping of groundwater from these depths in the more urban areas and associated depletion of ions from the groundwater at shallow depths from increased crystallisation, while showing the expected behaviour at larger, unaffected depths. Furthermore, anthropogenic modifications to the ground in residential locations, which change natural recharge and discharge patterns, may influence the evaporative behaviour in urban areas additionally.

While there is a correlation indicated between water extraction from the shallow aquifer and the saline interface position in this aquifer, no direct influence from deep bore extraction on resistivity changes within the shallow aquifer could be found. Nevertheless, the shallow coastal aquifer at both survey locations is subject to seawater intrusion related to increased abstraction from many registered and unregistered private shallow (3-20 m) wells in these two groundwater zones (an estimate of 6-7000 $\mathrm{m}^{3}$ /day only in the Waikanae groundwater zone during peak summer (Gyopari et al., 2014)). As a result, groundwater salinity should be monitored vigorously in order to timely register any deterioration in freshwater quality and take appropriate measures to avoid salinization of the shallow aquifer by all means.

Resistivity changes are usually within $\pm 40 \%$ and similar to the values that have been reported by De Franco et al. (2009) in their saline intrusion study in northern Italy. They found a significant time lag for deeper sections ( $>15 \mathrm{~m}$ depth) in the phreatic aquifer compared to the surface layer at that field site which they accounted to artificially controlled freshwater levels at the field site. Their study was conducted in a similar hydrogeological environment, demonstrating that the findings of such DCR monitoring studies can, to a certain degree, be applicable to similar geological and hydrological environments. Furthermore, when comparing the varying rainfall patterns between the two monitoring years in Figs. 2.3 and 2.7, with a 
reasonably consistent overall seasonal resistivity behaviour observed between the two groundwater zones, it becomes evident that short-term rainfall fluctuations only have a minor influence on the overall aquifer state. Long-term recharge is much more important to changes within the shallow coastal aquifer. Nevertheless, the fact that similar systems can behave differently, as demonstrated in this study, suggests that more extensive field studies and combinations of various field information are needed in order to find connections which are valid on a much broader scale. 


\section{CHAPTER 5}

\section{The role of the tides - analysis of tidal geoelectrical time series for periodic seawater mixing}

"The only reason for time is so that everything does not happen at once."

Albert Einstein, physicist and Nobel Prize winner (1879 - 1955)

In this chapter I investigate time series behaviour of DC resistivity data with regard to temporal tidal changes in a diurnal cycle. I will first introduce the aim and the resistivity data sets that have been used for this study. Then I give an overview of data analysis procedures, which will lead me to presenting and discussing the results that were produced by the non-statistical and statistical methods applied to the resistivity data. A summary will be provided at the end of this chapter outlining implications of the tidal time series study. Appendix $\mathrm{C}$ will then investigates a seasonal component of tidal dynamics as an extension of this work. This chapter is under revision as a paper with Water Resources Research: E. Sutter and M. Ingham. Time lapse DC resistivity measurements over a tidal cycle - using time series analysis techniques to infer preferential flow paths and saline mixing in a shallow coastal aquifer. 


\begin{abstract}
Saline intrusion research using geoelectrical time-lapse monitoring is often directed towards imaging the saltwater-freshwater boundary and the amount of seawater mixing within a coastal aquifer. However, these time series can contain additional information about subsurface hydrologic properties like hydraulic conductivity and permeability variations which are crucial elements in coastal groundwater management. In this feasibility study, a DC resistivity time series from a 24-hour tidal time-lapse monitoring survey of a shallow coastal sand and gravel aquifer in New Zealand has been analysed for recurring patterns of seawater mixing in different portions of the aquifer. Thereby, correlations between tidal and groundwater stage, respectively, and model resistivity within the near-shore aquifer portion could be demonstrated by means of resistivity ratios and time series analysis techniques such as dynamic time warping and autocovariance methods. The aquifer divides largely into three parts with the tidal run-up zone making up the portion of the aquifer that shows the largest fluctuations with time, which is directly related to tidal wave action, short time lags to tidal stage and small resistivity periods. This area also typically does not exceed absolute resistivity changes of more than $5 \Omega \mathrm{m}$. Parts of the resistivity model that are further inland and at greater depths either show a positive or negative relationship to tidal stage. However, they also show a larger period than the tidal signal, which is similar to the dominant period found from groundwater level fluctuations in several wells of the area. Although clearly showing a change in resistivity and hence indicated changing salinity over time, the bulk portion of the imaged aquifer section appears to be mainly dominated by freshwater dynamics rather than tidal forcing. The source of the groundwater and resistivity period could not be be determined, though.
\end{abstract}




\subsection{Introduction}

Several hydrological numerical and field studies have been carried out in previous years in order to study the influence of natural periodic events on groundwater temperature in the freshwater-saltwater transition zone (Gunawardhana and Kazama, 2009), near-shore circulation zone dynamics (e.g. Kim et al., 2006; Robinson et al., 2007; Kuan et al., 2012; Heiss and Michael, 2014), submarine groundwater discharge (Taniguchi, 2002) and saline interface characteristics (Levanon et al., 2016). A more extensive overview of research on the tidal influence in coastal aquifers is also given in Section 1.2 of this thesis. In order to study periodic events usually shorter or longer time series are modelled or acquired in the field. Thereby different methods of time series analysis are used. In time series analysis, the dependence among observations at different points in time is studied (Diebold et al., 2006). These can range from simple difference- or ratio maps between time steps through to more sophisticated methods like frequency spectrum analyses. A common method in such time-series studies has been the application of cross-correlation in order to determine the relationship between periodic events occurring across two time series. For example, this method has been used by Kim et al. (2006) to determine a relationship between semi-monthly and semi-diurnal variations in electric conductivity (EC) and tidal stage data. By cross-correlating EC data from different well depths these authors could distinguish in which aquifer layer (coarse sand rather than fine sand) saline intrusion was dominant, and could rule out correlation of the EC time series with rainfall - a very useful result in terms of understanding aquifer parameter and recharge characterization. In a recent study, among other statistical methods, Johnson et al. (2012) discussed the application of cross-correlation of 3D DC resistivity monitoring data, collected in the vicinity of the Columbia River (USA), with river stage data. This is, to my knowledge, currently the only study cross-correlating geoelectrical inversion results with groundwater stage data. By combining time series and time-frequency analysis methods Johnson et al. (2012) were able to isolate a hydraulically more conductive paleochannel.

Although using cross-correlation in order to find time series inter-relationships, especially time lags between two series of interest, appears to be a popular tool in recent studies, the method comes with a variety of complications. For example, both time series need to be sampled at the same interval, which therefore prevents irregularly sampled data from being analysed 
by cross-correlation. Also, the assumption of the two signals being of equal period (i.e. copies of each other with a shift in time) needs to be satisfied. This may not be true for real-world problems such as resistivity changes in an aquifer relative to a tidal or other cycles, because the pore matrix presents a certain resistance towards water circulation (as opposed to for example surface water in an estuary). This problem is illustrated in Fig. 5.1 where two sinusoidal signals with a time shift and the same period are plotted in (a), and two signals of differing period are plotted in (b). In the case that the two time-shifted signals have the same periodicity, then the time lag $(\tau)$ between the signals will be the same at any time. However, if the periods of the signals are different (e.g. 6 hours vs. 8 hours) then the time lag found by cross-correlation will be different depending on the observational time window. Hence, cross-correlation may not be the optimal tool for investigating two time series with respect to their time lag if they have irregular or differing temporal behaviour. For such cases the dynamic time warping (DTW) method can be used in order to measure the similarity between two periodically differing time signals. The method is explained in Section 3.5 and finds wide applicability in speech recognition, but has also been applied to geophysical problems in satellite image analysis (Petitjean et al., 2012) and seismic image processing (Hale, 2013).
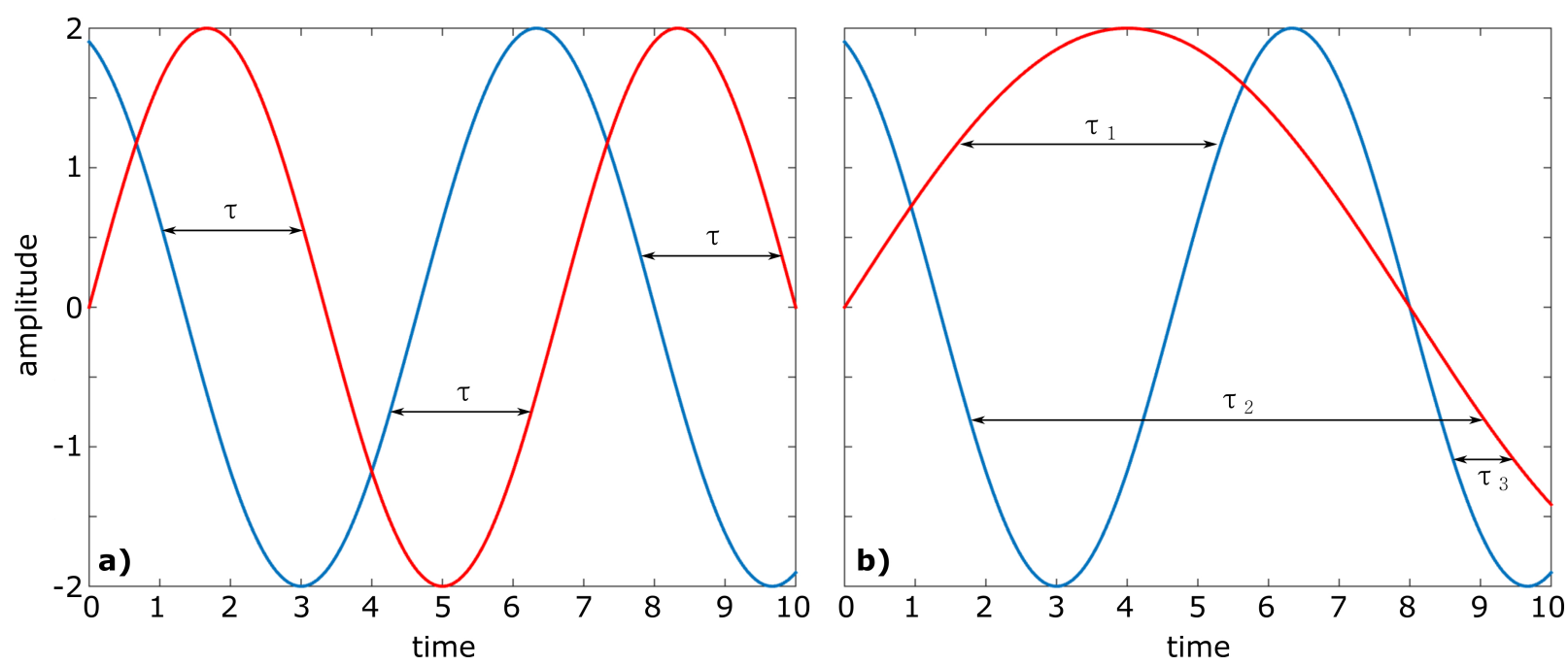

FIGURE 5.1: Two examples of two sinusoidal signals at a time shift with a) the same frequency (period) and b) different frequencies. It becomes quickly clear that the time lag between the signals in (a) is always constant, while the time lag between the signals in (b) differs by large amounts depending on what part of the time series is examined.

While ratio- or difference maps are very easy-to-use tools in order to reveal intrinsic temporal behaviour between time steps, these methods are often too simple to understand underlying temporal behaviour, are only applicable to two images at a time, and are subject to ordering 
priorities (Petitjean et al., 2012). Furthermore, it becomes very cumbersome to image the results if the time series reach lengths of more than about six time steps. DTW works by dilatation and stretching the two signals to their optimal fit and gives a distance between them. This distance can be interpreted as a cost, where an optimal (or minimal) warping cost (OWC) occurs for two very similar signals. However, no information about signal periods is obtained by using DTW. In order to get the information of the unknown periodicity for the DC resistivity time series, autocovariance can be used (see Section 3.6). This is a well-known technique to obtain signal periodicities and is widely applied in various disciplines (e.g. stock market analytics, climate parameter variations, big-data mining) (Shumway and Stoffer, 2016). Combination of the above techniques has been used on the tidal time-lapse resistivity monitoring data sets presented in this chapter to find parts of the aquifer that react in a similar way throughout a tidal cycle and to gain an insight into hydraulic aquifer properties. The results presented in this chapter can be seen as feasibility examples in order to apply the discussed methods to longer geoelectrical monitoring data series in the future.

In the following sections the field data are introduced and procedures for tidal DC resistivity monitoring data analysis are described in more detail. First, geoelectrical data processing and handling is discussed. This is followed by explanations about how DTW and autocovariance were used on these data sets. The main part of this chapter concerns presenting and discussing the results from the tidal time-lapse monitoring study in the Waikanae groundwater zone with the different time series analysis techniques introduced during the course of this discussion. This chapter is structured around the Waikanae data set, however, several shorter semi-dirunal monitoring data sets were acquired in Te Horo and analysed in a seasonal context as discussed in Appendix C. For both locations signal periodicity, obtained by fast Fourier transforms, of tidal and groundwater stage, as well as atmospheric pressure, is additionally discussed in order to investigate dependencies of the resistivity signals. At the end of this Chapter the most important aspects of time series analysis applied to tidal DC resistivity monitoring data sets are summarised. 


\subsection{Procedures for data analysis}

The field data used in this study is part of a 24-hour DC resistivity time-lapse monitoring that was carried out along the Waikanae N2 profile (Fig. 4.1) with a sampling interval of one hour during 22-23 November 2015. Due to inversion code restrictions only 21 of the 24 hours could be analysed at one time. In order to be consistent throughout the thesis, it was decided to rather cull three hours of the monitoring series instead of using another inversion program. The electrode positions during the entire measurement cycle were not changed. Because the first four to eight electrodes would be standing in seawater during high tide, up to $1.8 \mathrm{~m}$ long stainless steel rods were used to attach the cables to at these positions in order to ensure that the electronics stayed above the seawater. The difference in resistance between the $1.8 \mathrm{~m}$ and regular $0.35 \mathrm{~m}$ long electrodes was measured to be in the order of $0.2 \Omega$ and should therefore have no significant influence on the measurements. In order to image the expected small changes with time, a two-meter minimum spacing (Wenner- $\alpha$ array) was chosen to enhance spatial resolution, leading to a total of $78 \mathrm{~m}$ transect extent. The groundwater level measured in well R26/6287 (location see Fig. 4.1) was on average $2104 \mathrm{~mm}$ during the resistivity monitoring period, and the tidal range during the 21 hours amounted to a mean of $1.30 \mathrm{~m}$. The initial aim of monitoring resistivity changes during a tidal cycle was to determine whether resistivity changes within the very near-shore part (within approximately $100 \mathrm{~m}$ from the shore line) of the shallow coastal aquifer are a consequence of tidal stage fluctuations. However, such time series can likely also be used to infer aquifer properties such as hydraulic conductivity. The above mentioned statistical methods (DTW and autocovariance) are therefore used in addition to the classical resistivity ratio maps on the tidal monitoring data sets in order to further investigate differing hydraulic properties within the aquifer.

\subsubsection{Time-lapse DC resistivity filtering and inversion}

For each time step of a monitoring period, data quality was assessed individually and measurements were removed where they exceeded $10 \%$ of the relative difference between normal and reciprocal values or had an injected current of less than $0.01 \mathrm{~A}$. If a data point was removed in any of the time steps of a data set, this point was also removed in all of the other time steps of 
that data set in order to have a consistent set of measurements comparable over all time steps. A total of 9 data points were removed from each time step data set. This is only $4 \%$ of the original data and considered insignificant. A noise estimate was added to all measurements of the same time step accounting for the average reciprocal error (1.5\%) obtained from three reciprocal measurements taken during the entire monitoring (Slater et al., 2000; Robert et al., 2011), and the average repetition error of all measurements taken within each time step. This led to an overall error estimate of $2.27 \%$ for the complete data set.

Inversion was done with the commercially available software Res2DInv (32-bit version) (Geotomo, Malaysia) in a difference time-lapse style inversion (LaBrecque and Yang, 2000), where the first time step acts as a baseline model and each following time step is inverted with reference to this model. This achieves a more robust inversion process with less pronounced inversion artefacts (Ogilvy et al., 2009). Topographical data were added into the inversion process in order to correct for vertical deviations. The topography was measured along the DC resistivity profile using a differential GPS device (Trimble GeoExplorer) which resulted in a vertical accuracy of approximately $20 \mathrm{~cm}$. In order to account for sharp resistivity changes across the saltwater interface, the least-squares inversion (deGroot-Hedlin and Constable, 1990; Sasaki, 1992) used a robust $\left(l_{1}\right.$-norm) constraint on both data and model. This means that the absolute difference between measured and calculated apparent resistivity values is minimized, rather than the squared difference as is the case for the smooth $l_{2}$-norm constraint. This will give less weight to outlier/extreme data points. Forward modelling was carried out on a rectangular mesh with the finite element method to allow for topographical distortions. A time-lapse damping factor of 0.85 was introduced in order to account for differences in the individual time steps but still achieve a relatively high similarity between the time-lapse inversion models (Loke et al., 2006). The inversion was set to stop once the root mean square (RMS) error would fall below the noise level or the change in RMS error between iterations did not exceed $0.5 \%$. This was satisfied at an RMS value of $4.1 \%$.

Because parts of DC resistivity inversion models can be constrained by the model rather than the data, these parts have to be identified and blanked out for interpretation. This study also used the DOI indexing method for resistivity model appraisal. Details about this method can be found in Section 3.2.3. The DOI index values for the tidal inversion models were bimodally 
distributed, which means that their distribution peaked around two maxima ( 0 and 1$)$. Hence, the cut-off factor could be estimated using the statistical histogram analysis method discussed in Deceuster et al. (2014). The DOI index depends on the individual inversion model and is therefore different for each time step. For ratios, the average DOI index of the two relevant time steps for each model element was taken to blank out unreliable model parts.

\subsubsection{On displaying long 2D geoelectrical time-lapse series}

Geoelectrical monitoring surveys can contain several tens to hundreds of time steps, each of which consists of a full data set and its own inversion model. Displaying more than about five or six such models on the same page rapidly becomes cumbersome and details become hard to read (as is demonstrated within this chapter). Imaging resistivity ratios is very common for time-lapse geoelectrical data sets and can help to significantly reduce the number of images. However, if simple ratios between two periods of time (i.e. between summer and winter) are shown some of the temporal information is ignored (as demonstrated in Chapter 4). The time series presented in this chapter contains 21 time steps and the changes between those time steps are expected to be relatively small $(<20 \%)$. As leaving out information can lead to misinterpretation, but having pages of inversion models can itself be hard to read and interpret, another way of displaying such data sets has been developed. This involves taking the results of the time-lapse inversion and plotting the entire time series of resistivity values for each individual model cell. All such plots are then included on a single plot of the model space. As is illustrated in Fig. 5.2 this explicitly reveals portions of the aquifer where the temporal behaviour of resistivity is the same.

In order to only use cells of the inversion model that actually contain seawater (as the study is interested in salinity changes tied to tidal fluctuations), the plots are restricted to cells exhibiting a resistivity value below a threshold that was determined by defining the zero-percent seawater resistivity based on Archie's law (Eq. 3.28) using the formation factor $F$ of 2.75, as determined for the study area by Wilson et al. (2006), and the measured electrical resistivity $\rho_{f}$ of the river water in the area $(0.02 \mathrm{~S} / \mathrm{m}=50 \Omega \mathrm{m})$. Although fluid conductivity is directly proportional to salinity, groundwater salinity is not only a function of the sodium chloride concentration of the fluid, but a measure of total ions in solution. Hence, freshwater does not have zero conductivity 


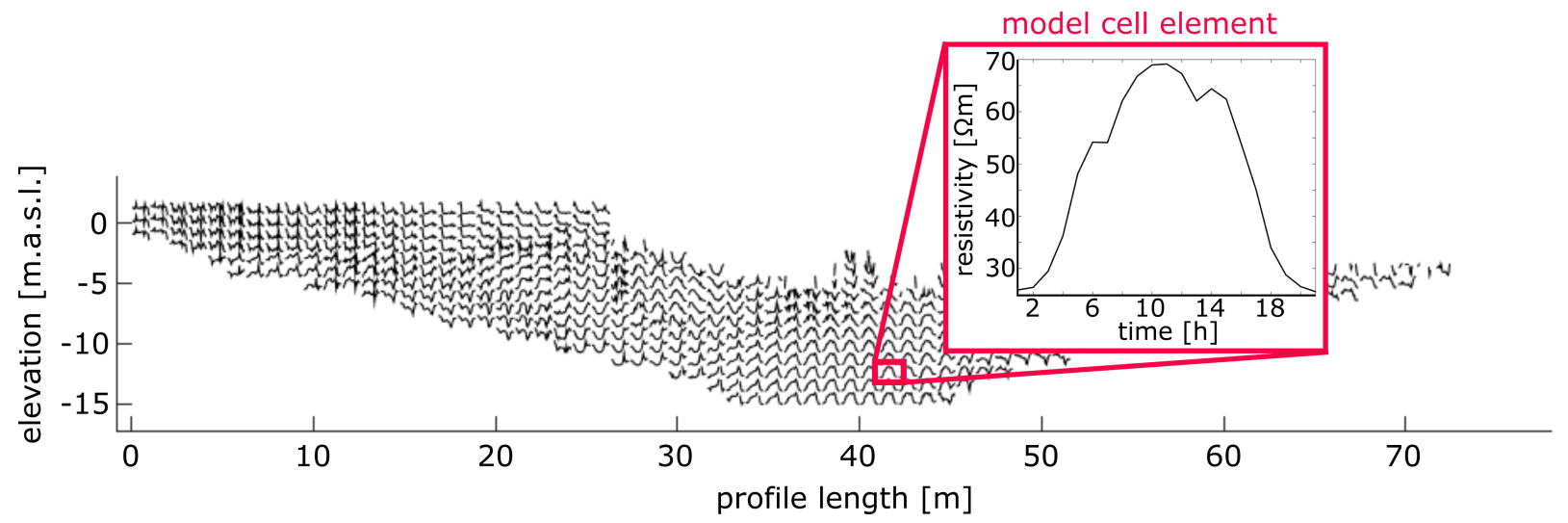

FIGURE 5.2: Example plot of long time series. Each model cell element shows the amplitude (i.e. resistivity) on the y-axis, and the time on the x-axis. All of them are then plotted in the model space, illustrating the portions of the profile that show similar temporal behaviour. Note, model resistivity scales differ for each cell.

and therefore the cut-off factor of $50 \Omega \mathrm{m}$ for river water resistivity has been regarded as being a representative number for the freshwater threshold value. This results in a threshold bulk resistivity value $\rho_{b}$ of $140 \Omega \mathrm{m}$ at the study site and inversion elements exceeding this value were not taken into account for analysis. For computational reasons when plotting the coloured version of the time series plots (e.g. Fig. 5.6(b)), the model cells that have only shown values lower than the threshold for part of the monitoring time (i.e. bordering elements between fresh- and seawater mixed groundwater) were also blanked out. Furthermore, model cells that have been regarded as constrained only by the model rather than the data through DOI index analysis have been omitted from this analysis.

It is clear that not only do cells differ in the magnitude of resistivity, but they also behave markedly differently in terms of resistivity change with increasing monitoring time. As it is the hydraulic properties of the subsurface that ultimately control the movement of groundwater in response to tidal or freshwater changes, the different characteristic shapes of the curves can be interpreted as resulting from the cells having different hydraulic properties. The proposed plot style is used in the following sections.

\subsubsection{Inferring hydraulic ground properties with statistical methods}

As implied in the previous section, long-term resistivity monitoring time series can isolate different subsurface parts of an aquifer with differing temporal behaviour. In addition to 
visualising these areas, as can be achieved with the method described above, it would be beneficial to know what other properties these regions have. With regard to saline mixing dynamics investigated during a diurnal tidal cycle, the time it takes the aquifer to adjust its resistivity to changes in tidal stage (i.e. high tide versus low tide) is of prominent interest because it will tell indirectly how hydraulically conductive the material in different aquifer areas is (assuming that the sedimentary matrix is stationary throughout the measurement cycle). A measure of this can be accomplished by using dynamic time warping (DTW), which was explained in more detail in Section 3.5. In order to estimate the similarity of the aquifer state, in terms of resistivity, to tidal or groundwater stage, the resistivity time series have been used together with these stage time series for the same monitoring time period. The resistivity values obtained for each inversion model cell have been converted into its inverse, electrical conductivity. The tidal stage time series with respect to the lowest possible tide at the study sites were calculated from predicted times for high- and low tide stages at the Waikanae River mouth (https://www.niwa.co.nz), using

$$
h=h_{1}+\left(h_{2}-h_{1}\right)\left[\frac{(\cos (A)+1)}{2}\right]
$$

with

$$
A=\pi\left[\left(\frac{\left(t-t_{1}\right)}{\left(t_{2}-t_{1}\right)}\right)+1\right]
$$

where $h_{1}$ and $t_{1}$ are the height and time of the tide (high or low) preceding time $t$, and $h_{2}$ and $t_{2}$ the one (low or high) following time $t$. The height of the tide at time $t$ is then given by calculating $h$ with Eq. 5.1. Note that $A$ is given in radians and all the times are in decimal hours. Tidal time series were calculated at one-hour intervals starting and ending at the same times as the DC resistivity data set. Groundwater stage data was taken from the GWRC monitoring data base for well R26/6287 located south of the resistivity transect (see Fig. 4.1), also using an hourly sampling rate.

Knowing how well two signals correlate only holds half the information needed for a comprehensive hydraulic conductivity estimation, however. Another important characteristic is the time it takes until the same state of a subsurface area is reached again - the period of the resistivity signal. This was determined for the resistivity time series by using autocovariance, as 
explained in Section 3.6. For the statistical analyses (DTW and autocovariance) the data sets were transformed so their time series were distributed normally (i.e. zero mean and standard deviation of one). This is useful in pattern recognition as the analyses are insensitive to small fluctuations of the signals (i.e. outliers and errors) but determine the overall behaviour (Rojas, 2010). Also, the amplitudes of the tidal, groundwater and electrical conductivity signals differ from each other. Transforming them removes any effects due to this difference. In addition, to ensure normal distribution of the time series, they were also smoothed with the moving average method over a window of three hours in order to remove random or irregular components in the resistivity time series. Using this procedure helps in isolating a cyclical trend. The effects of these time series alterations are illustrated for the tidal data set and two inversion model cells from the Waikanae N2 tidal monitoring series in Fig. 5.3. While the scales become comparable using the transformation to normal distribution, smoothing evens out rapid changes in the signals. The characteristics of the original data are preserved after the time series modifications.

As the autocovariance looks for positive dominant peak correlation in the signal, it is expected to show a time of close to 12 hours for the tidal time series as this is the approximate time usually taken for a full tidal cycle. Resistivity monitoring data sets are expected to show similar cycle times as the tidal time series in case the resistivity changes are dependent on gravitational forcing. However, if the resistivity signal is dependent on other forces (e.g. groundwater), this should result in a different period than the one associated with tidal forcing. Also, it is likely that smaller cycle times will be observed in the shore proximal part of the aquifer and larger ones the further away the profile extends from the coast. Obviously, a cyclicity of the resistivity signal and similar shape as for the tidal time series is assumed in order for the statistical method to work. This is inferred by simply looking at the inversion model resistivity curves with increasing monitoring time for individual model cells (e.g. in Figs. 5.3(b) and 5.3(c)).

Similar expectations apply to the warping costs obtained by DTW. Model cells further away from the coast are generally expected to show larger optimal warping costs between tidal and resistivity signals than those located close to the coast and vice versa, smaller costs are expected between groundwater and resistivity signals further away from the coast. The same observations should be made for cells with lower and higher hydraulic conductivity, respectively, where cells having a high hydraulic conductivity should move seawater quickly and therefore 

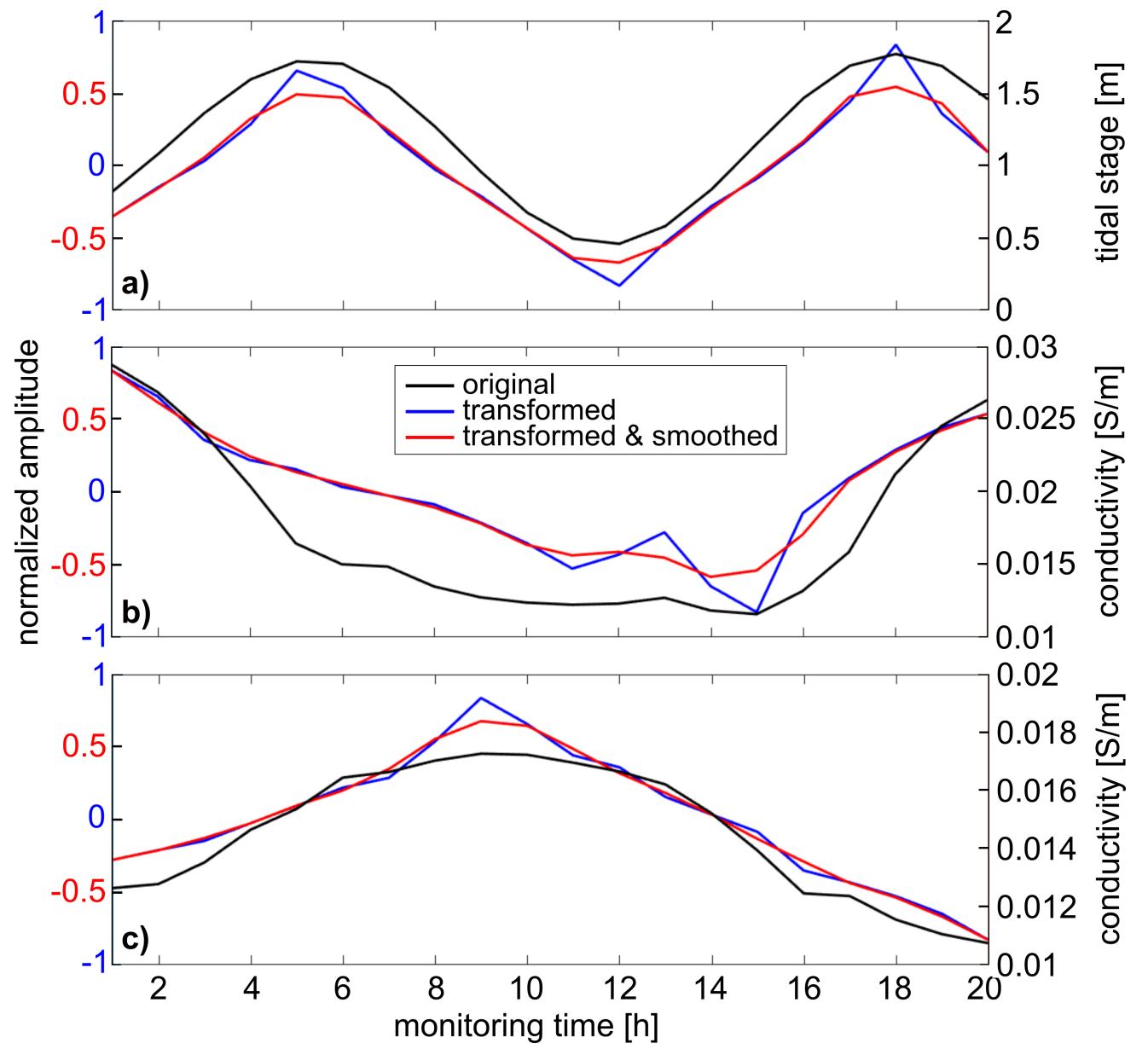

FIGURE 5.3: Example of the effects of transforming and smoothing time series. Black curves are the original data of the time series (note difference in scale on the right y-axes), in blue the transformed signals, and in red the transformed and smoothed signals. Note that the scale for the latter two is the same and displayed on the left y-axes. Data was taken for a) tidal time series, b) an inversion model cell with a decreasing then increasing conductivity trend, and c) an inversion cell with an increasing then decreasing conductivity trend.

having higher similarity to the tidal signal than to the groundwater signal. However, it has to be kept in mind that the time series of the data sets presented in Section 5.3 are generally rather short and could therefore image reactance to previous tidal stages. The results from the DTW and autocovariance analyses can be understood as a feasibility study for statistical methods applied to geophysical monitoring problems and aim to illustrate strengths and weaknesses of these applications. 


\subsection{Diurnal tidal cycle monitoring in the Waikanae ground- water zone}

In their high-resolution cross-hole resistivity tomography study, Morrow et al. (2010) imaged some significant movements (up to 7-8 m) of saline water in the near-surface mixing zone within a time frame of a few days and concluded that wave run-up and tidal head changes have a significant influence on saline intrusion in the study area around the Kapiti Coast. However, these changes were observed on a very local scale and appeared to be highly dependent on the tidal amplitude. They also could not detect such tidally influenced changes with their 5-m minimum spacing surface DC resistivity transect measurements at the same location. The results presented in this chapter investigate the temporal behaviour of the near-shore mixing zone in the shallow coastal aquifer with respect to a semi-diurnal cycle, using a 2-m minimum spacing in order to enhance spatial resolution. In the following, the discussion is split into two parts, first examining the resistivity signals using ratios between time steps and investigating the model space in terms of overall temporal behaviour with the new time series plot style. The second part discusses results from the DTW and autocovariance analyses in light with Fourier transforms of the tidal and groundwater signals. This is performed purely on a diurnal tidal cycle data set. In Appendix C relationships of the resistivity signal measured over several hours on different days throughout the year are presented in order to investigate seasonal influences on short-term resistivity changes.

\subsubsection{Tidal resistivity ratios and time series}

To quantify any possible tidal influences on the seasonal saline intrusion monitoring, hourly repeat measurements were carried out along the N2 transect over a 24-hour tidal cycle (diurnal tidal monitoring). Details on the survey were discussed in Section 5.2. Starting at the 50-m mark of the original (i.e. seasonal) N2 profile, distinct resistivity changes over time can be observed in Fig. 5.4 for part of the series. The penetration depth of this shorter but higher resolved profile only allows the top and middle layers of the shallow aquifer to be imaged. As for the seasonal measurements, no significant shift in position of the saline interface is apparent during the diurnal tidal cycle. However, changes in resistivity occur over the entire model space. 
An increase of resistivity in the middle part of the profile $(\sim 80-95 \mathrm{~m})$ occurs as the tidal stage moves from low to high and continues until about four hours after high tide. This then reverses into a gradual decrease of resistivity about 1-2 hours before the low tide becoming more and more conductive through to the next high tide. In the eastern and western parts of the profile, the opposite behaviour is observed. Assuming that a larger amount of saltwater intrudes the shore-proximal part of the aquifer at high tide and more freshwater is present during low tide, this suggests that circulation within this part of the aquifer in response to the tide is highly complex and that there is a spatially dependent time lag in the resistivity response relative to the tidal variation.

In a tidal monitoring study it is of interest to know the changes appearing between tidal extremes (i.e. low and high tide) or within a similar time period (i.e. within six hours). This is plotted for the N2 tidal monitoring profile in Fig. 5.5 where the ratios for subsequent tidal extreme stages are imaged. Very similar temporal behaviour patterns within the model are identified for both cases, showing an overall increase in resistivity from high to low tide within the middle part of the profile at around 80-95 m extent (marked A). Therefore, even though the resistivity ratios between adjacent time steps decrease in amplitude from high to low tide in Fig. 5.4(b), the global relationship is a clear increase in resistivity with falling tidal stage as is also indicated when comparing with inversion models in Fig. 5.4(a). The opposite is true for observations coming from low to high tide through time steps 11 to 18 in Fig. 5.4(b). This also shows in the Fig. 5.5 bottom image, which indicates a significant decrease in resistivity within this part of the aquifer for a ratio of HT/LT. An increase in resistivity with falling tidal stage and decrease of resistivity with rising tidal stage is quite intuitive as less or more seawater may intrude with a lowered or increased pressure from wave run up, respectively (these parts are marked $A$ in Fig. 5.5). However, there are several parts of the aquifer that show the opposite behaviour (marked $B$ ). They occur both west and east of the middle part in both Figs. 5.4 and 5.5 and illustrate the previously mentioned complexity of the aquifer response within the shore proximal zone as a potential consequence of spatially varying time lags which may be related to hydrological parameters.

It is very convenient to illustrate resistivity ratios between selected time steps in long resistivity monitoring data sets, but as has been shown in Chapter 4 for seasonal investigations, and is 


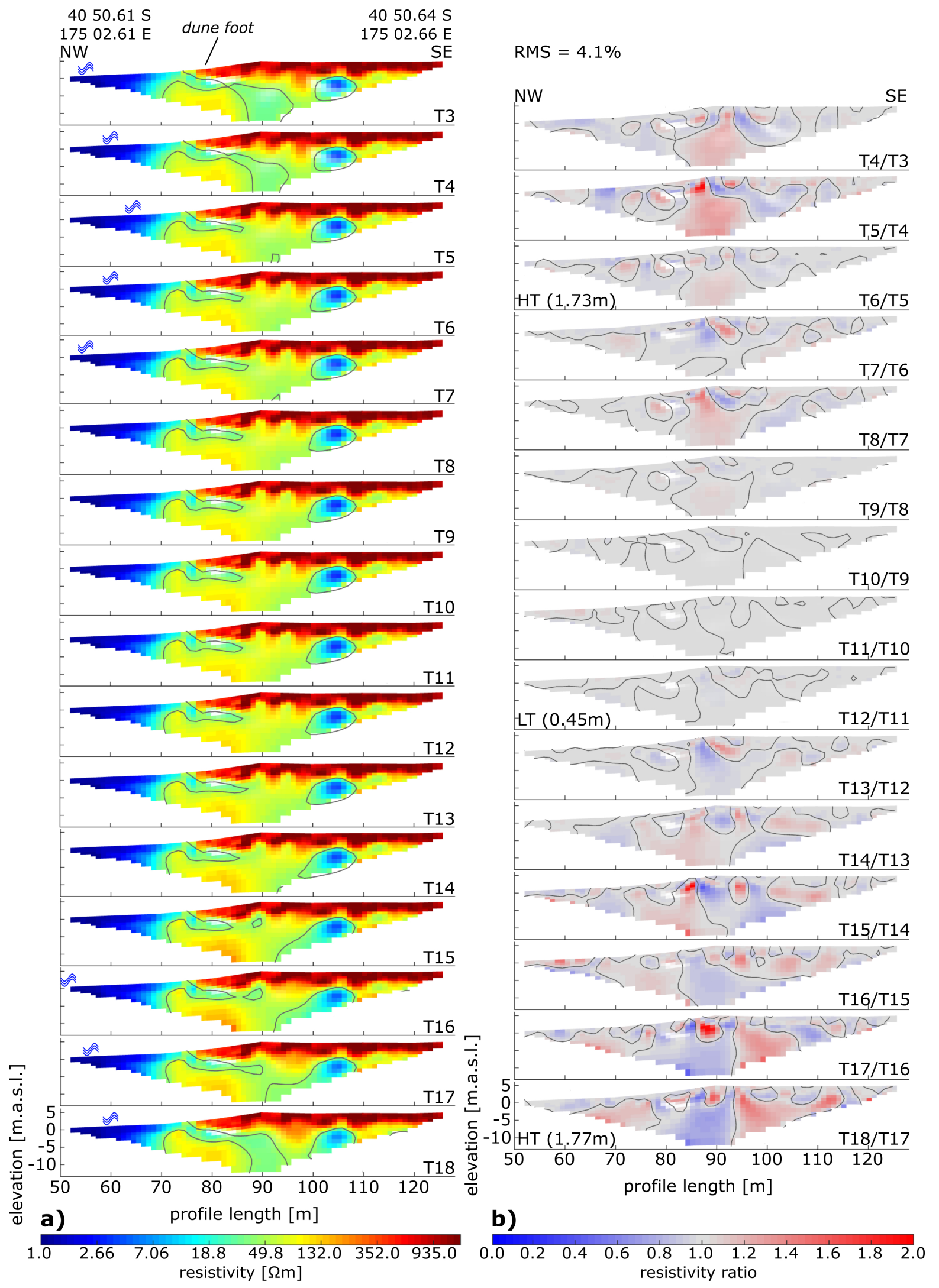

FIGURE 5.4: a) Inversion models for the 21-hour time series collected in November 2015 along profile N2 in the Waikanae groundwater zone (see Fig. 4.1), and b) the resistivity ratios between each consecutive time step. 
evident from comparison between Figs. 5.4 and 5.5, temporal information that may be of interest can get lost in such plots. Therefore, the time series were illustrated as individual line plots for each model element in Fig. 5.6(a) (inversion model resistivity versus time) for the first 21-hours of the complete tidal monitoring data set collected along profile N2. For better visibility of temporally similar modal cells, these can be statistically binned using the gradients between neighbouring data points of each time series in a model cell and comparing this to all other model cells. If a positive correlation of minimum $95 \%$ occurred, then two (or more) model cell time series were regarded sufficiently similar and coloured accordingly. This leads to Fig. 5.6(b), which may then be used to distinguish parts of the subsurface that act in a similar way to changes in the environment with time. Note that the colour scale for the latter plot type (in Fig. 5.6(b)) colours similarly behaving cells with the same colour. However there would be no correlation in colour between such plots for different profiles as the colours are chosen randomly. Another thing to note is that this similarity grouping has been done in terms of shape appearance of the time series only and cannot be compared in terms of magnitude. In general, Fig. 5.6(a) and (b) highlight parts of the aquifer with a similar temporal response as has been found from the resistivity ratios between tidal extremes in Fig. 5.5. However, there are differences between these illustrations due to the use of the entire time series and the resulting introduction of differing temporal behaviour categories in Fig. 5.6.

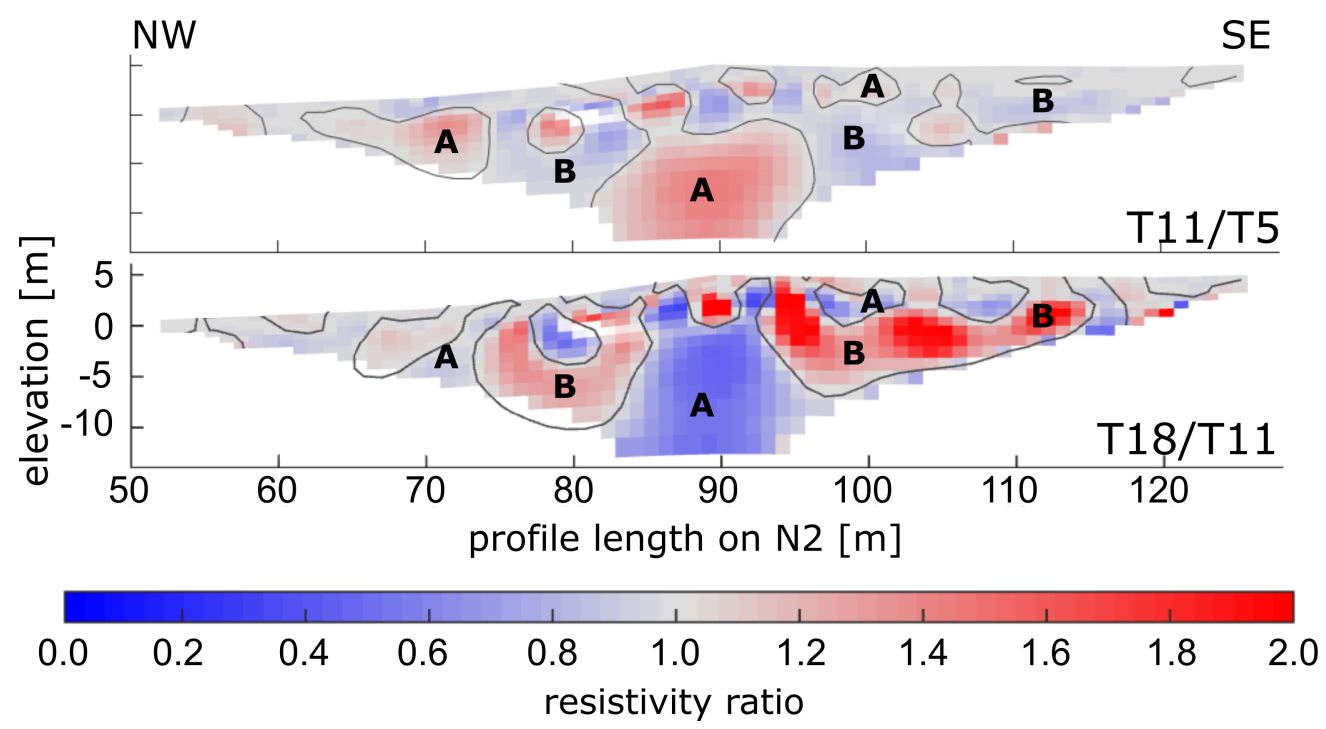

FIGURE 5.5: N2 21-hour resistivity ratios between subsequent high and low tides (top: LT1/HT1, bottom: HT2/LT1). Aquifer parts that show an increase of resistivity with decreasing tidal stage and a decrease of resistivity with increasing tidal stage are marked with $A$, whereas parts marked $B$ show the opposite behaviour. 


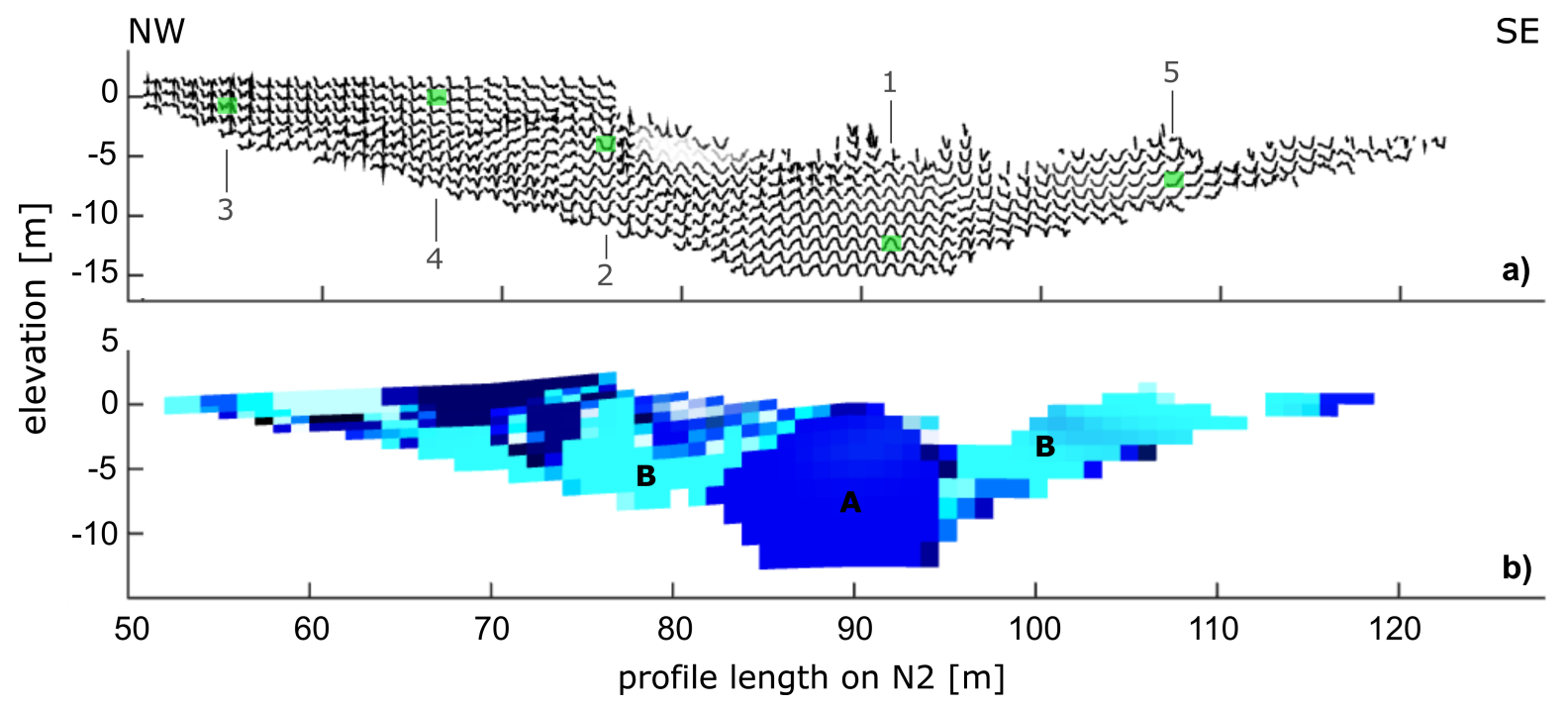

FIGURE 5.6: N2 21-hour time series shown as resistivity time series in a) (i.e. each model cell has time on the x-axis and resistivity amplitude on the y-axis), and coloured according to their temporal behaviour in b) (i.e. same colour equals same temporal behaviour of the resistivity). This allows to display the entirety of Fig. 5.4 in just one (or two) figure(s). Note that there is no topography included in (a).

Within the time series of the Waikanae N2 tidal monitoring profile shown in Fig. 5.6(a) five characteristic curves can be distinguished. Examples of these are marked in Fig. 5.6(a) with green colouring. They are magnified in Fig. 5.7 and can be described as:

1. Bell-shaped curves showing an increase of resistivity with decreasing tidal stage and a decrease in resistivity with increasing tidal stage (marked $A$ ),

2. U-shaped curves behaving in the opposite way to the bell-shaped curves (marked $B$ ),

3. Fluctuating curves which show apparently random changes in resistivity over a tidal cycle,

4. L-shaped curves having a sharp decrease in resistivity just before high tide and then remain level, and

5. J-shaped curves with a relatively uniform resistivity at first which quickly increases after low tide (also marked $B$ ).

While bell-shaped curves ( 1 or $A$ ) are mainly found in the middle part of the imaged aquifer section ( 80 to about $100 \mathrm{~m}$ profile length on N2), U-shaped time series ( 2 or $B$ ) can be identified to the west and east of this area and typically at shallower depth. A lot of fluctuation in resistivity (3) occurs within the wave run up zone between 50 and $65 \mathrm{~m}$ profile length on 
N2. Above the mean high tide mark from about 65 to $75 \mathrm{~m}$ and at shallow depths, L-shaped curves (4) are dominant, whereas J-shaped time series (5 or B) are found from about $100 \mathrm{~m}$ extent in the eastern part of the profile. These were binned together with the U-shaped curves possibly because a small upward tail at early monitoring hours may lead to being regarded as mathematically similar to a U-shape in Fig. 5.6(b).
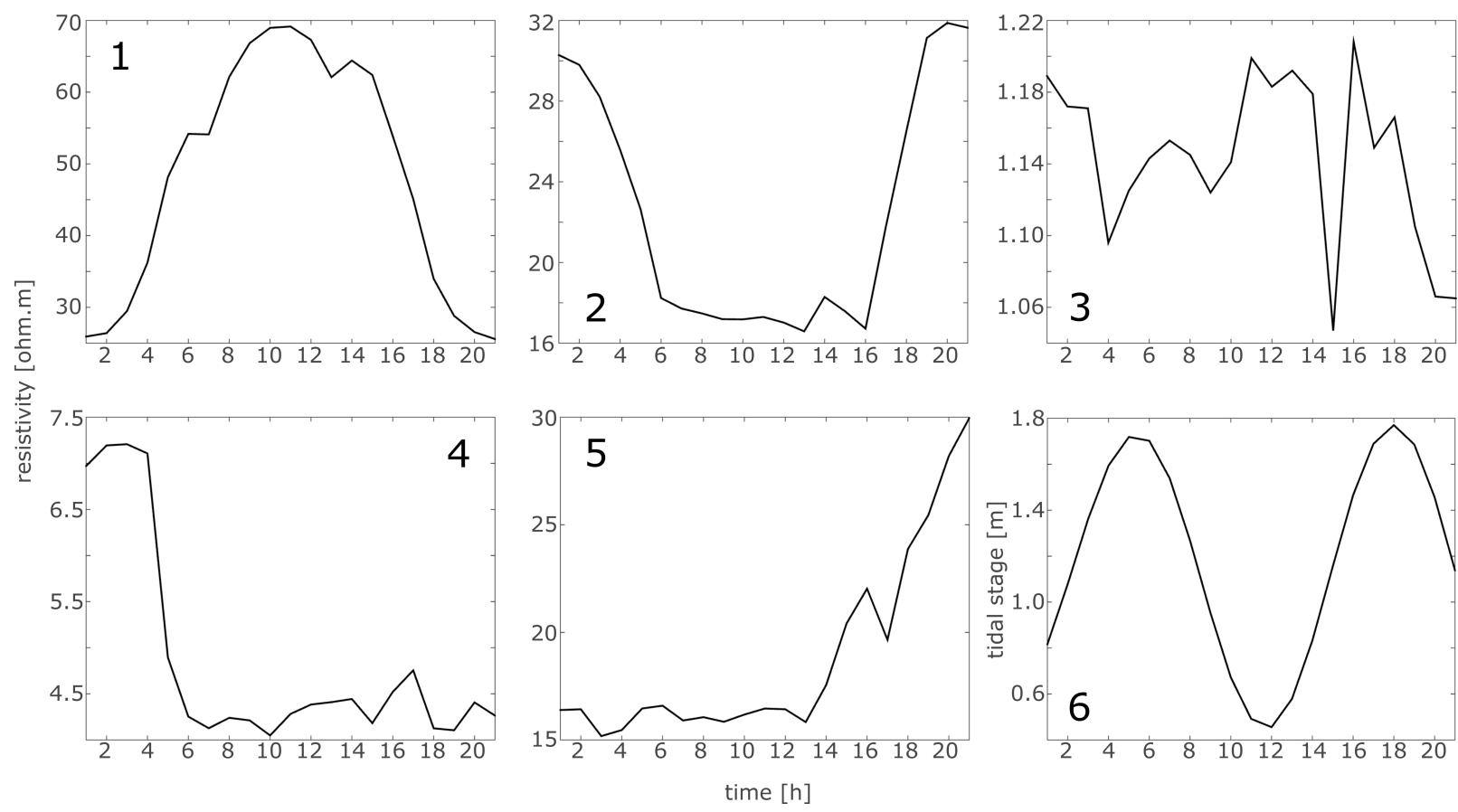

FIGURE 5.7: The five characteristic curve shapes identified in the Waikanae N2 inversion model time series. 1) bell-shape, 2) U-shape, 3) fluctuating, 4) L-shape, and 5) J-shape. 6) shows the tidal stage signal during the DC resistivity monitoring at the field site for reference.

Model cells with curve type (1) are expected to react quickly to changes in tidal stage relative to model cells featuring curve type (2), because they show the expected increase in resistivity with decreasing saline water content in the aquifer during falling tide and the reverse behaviour for a rising tide. However, they also indicate a considerable time lag and a longer signal period as compared to the tidal signal and therefore potentially show curve type (2) if a different monitoring window is chosen. A U-shaped curve on the other hand indicates a larger time lag as it exhibits a decrease in resistivity with assumed decreasing salinity during outgoing tide and also indicate a signal period larger than the one observed for the tidal stage. Fluctuations in the wave run up zone as indicated by type (3) curves are expected as waves wash in and out on the beach leading to small changes within short time spans. Model cells with this characteristic are mainly found in the wave run up zone and only rarely within the aquifer. L-shaped time series are found in the shallow area between mean high tide mark and unsaturated surface sands, 
and are therefore not directly in the zone of influence of the wave run up. However, this part of the aquifer lies in the upper mixing zone (see Fig. 5.4(a)) and the low resistivity suggests that these cells are at least partially saturated with relatively saline water. Therefore, close to high tide, more seawater mixing with these cells and fully saturating them, leads to a sudden decrease in resistivity. The desaturation after high tide may be a slower process indicated by the constant resistivity over a long time span. In general, unsaturated soil and sediments show a faster infiltration process than saturated ones due to the affinity of sediment particles to water (Heath, 1983). As a consequence, seawater may intrude at a much faster rate into these regions and subsequently migrate into the saturated zones of the aquifer more slowly. J-shaped curves found in the eastern part of the profile, which are also identified as being within $80 \%$ similar to the U-shaped curves, may be parts of the aquifer that take a longer time to adjust to tidal head changes, possibly due to their location further away from the beach face, but perhaps also because of lower hydraulic conductivity as may be expected to be the case for the U-shaped time series model cells during the chosen monitoring window.

It is also interesting to note that the highest resistivity changes occur in zones where bellshaped curves are found ( 1 or $A$ ). These changes amount to as much as $65 \%$ within a 21 hour span. Curve types (2), (4) and (5) show resistivity changes of up to about $50 \%$, whereas those for the near-shore fluctuating parts of the aquifer (3) only experience resistivity changes in the range of around 10 to $15 \%$ during the monitoring period. However, the absolute resistivity changes in some of the model cells are relatively small $(<5 \Omega \mathrm{m})$, which may be regarded as insignificant with regard to possible inversion model error and artefacts. This applies mainly to curve types (3) and (4), which are found in the direct influence zone of sea wave wash up. It is likely that these parts of the aquifer are to a large degree constantly saturated with seawater and that therefore the absolute changes in resistivity are very small and possibly not properly resolved in the inversion models. Hence, model cells with time series variations of less than 5 $\Omega \mathrm{m}$ were excluded from the statistical analysis in the following section.

In order to further illustrate complexity of the problem and outline inferred differences in subsurface hydraulic conductivity, groundwater movement can be implied from the time-lapse resistivity model plots (in Fig. 5.4(a)) as shown in Fig. 5.8. The changes in the contour lines (black arrows) of the $10 \%, 1 \%$ and $0 \%$ seawater mixing resistivity contour lines $(6,45$ and 140 
$\Omega \mathrm{m}$, respectively - see further explanation regarding calculation of these values in Chapter 6) suggest the groundwater movement (perforated arrows) shown in Fig. 5.8 during a falling (Fig. 5.8(a)) and a rising (Fig. 5.8(b)) tidal stage. This suggests a time delay with more saline mixing apparent for a rising tide as opposed to a falling tide that already experienced the maximum tidal range. A delay time for seawater intrusion is also implied due to the fact that the contour lines do not strictly change their movement direction (black arrows) at low and high tide boundaries but extend at either side of the tidal extremes. It is unsurprising that there is no immediate reaction to an increased seawater pressure during high tide given the estimated generally low hydraulic conductivity of the coastal aquifer.

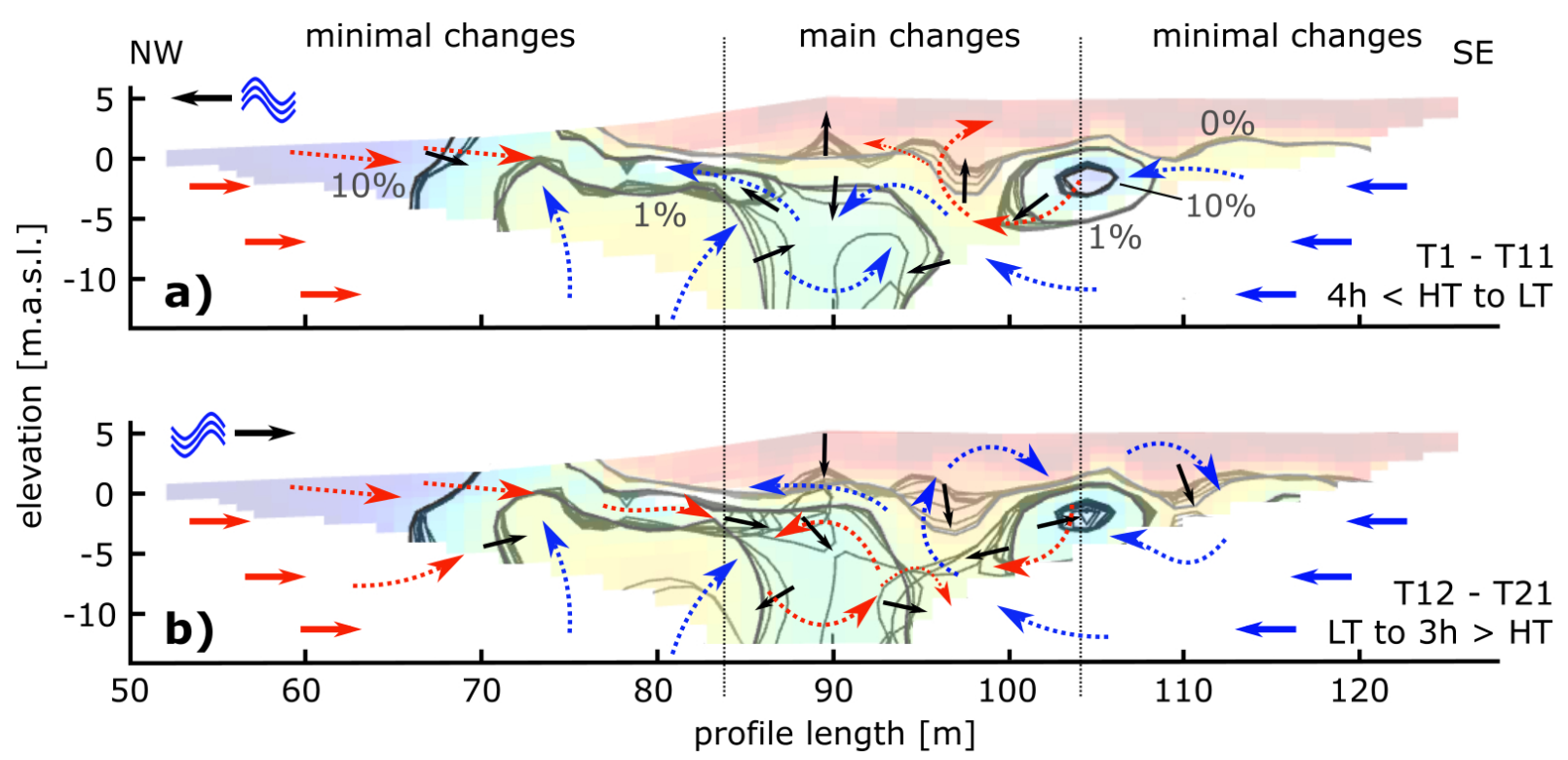

FIGURE 5.8: Water movement (perforated arrows) for the situations of a a) falling (four hours before high tide to low tide) and b) rising (low tide to three hours after high tide) tidal stage within the aquifer as indicated by changes in the $10 \%(6 \Omega \mathrm{m}), 1 \%(45 \Omega \mathrm{m})$ and $0 \%(140$ $\Omega \mathrm{m})$ seawater mixing contours from the 21 time-lapse inversion models of the DC resistivity monitoring in Waikanae. Red and blue solid arrows indicate relative hypothetical flow direction of seawater and freshwater, respectively. Black arrows mark the direction of the contour lines with increasing time

From Fig. 5.8 it becomes evident that the imaged aquifer portion can be divided into three parts where the main changes in resistivity occurring over time are found in the middle part and only minimal changes are observed on either side of this section. The reason for this may be that the north-western and south-eastern parts of the aquifer are mainly seawater and freshwater dominated, respectively, while in the middle mixing of the two fluids is dominant and adds complexity due to both density differences between sea- and freshwater and to a possibly differing hydraulic conductivity associated with changes in geologic features. In the situation of a falling 
tide in Fig. 5.8(a) freshwater flow appears to be dominant throughout the aquifer and leading to a general increase in resistivity in the middle part at around $90 \mathrm{~m}$ profile length. A local resistivity decrease during this time at around $100 \mathrm{~m}$ profile length may be due to a transfer of more saline water from an inclusion between 100 and $110 \mathrm{~m}$ as a result of freshwater pressure in the seaward direction. When this situation reverses to a rising tide as shown in Fig. 5.8(b), the delayed seawater entering the aquifer further inland has caught up and now reverses flow into the lower resistive zone at $90 \mathrm{~m}$ leading to the observed decrease in resistivity in this area. Freshwater flow from the south-eastern side continues and is now forced upwards at around 100 $\mathrm{m}$ profile extension, thereby increasing the resistivity in this area again. This can most probably be accredited to density contrasts between the two liquids where the more saline water is forced to lower depths within the geologically different zone at $90 \mathrm{~m}$ and allowing freshwater to flow vertically upwards in the mixing zone area. The observed delay of resistivity change in response to the tidal signal may also mean that tidal forcing is not the main driver of these changes.

The observations from the water movement analysis above match well with those observed by Ataie-Ashtiani et al. (1999) in their modelling study on tidal effects on seawater intrusion in coastal aquifers. They identified a combination of density- and tidal activity driven circulation close to the coast that significantly changes for situations of rising and falling tides. It is long known that the mixing zone shape and extent are a function of aquifer layout, hydraulic conductivity, groundwater in- and outflow, and tidal stage oscillations (Ataie-Ashtiani et al., 1999). In case of an unconfined, sloping beach aquifer seawater also infiltrates from the top during high tide leading to a larger portion of the aquifer being intruded by seawater. The lower the angle of the beach the more intensified this effect will be (Ataie-Ashtiani et al., 1999). For an aquifer with a low hydraulic conductivity $(K=8.4 \mathrm{~m}$ /day which is similar to the estimated overall hydraulic conductivity of the shallow coastal aquifer of about $5 \mathrm{~m} /$ day) Ataie-Ashtiani et al. (1999) modelled the flux of groundwater for situations of a rising and falling tide and they observed an increased inland intrusion of seawater along with a widening of the mixing zone for lowered freshwater pressure. Regardless of freshwater flow, they also showed that the intrusion depth and thickness of mixing zone radically changes when tidal fluctuations are included in the model and tied that to changes in the flow pattern and velocity of groundwater flow close to the coast - something that is strongly suggested by the groundwater movement analysis for the diurnal resistivity study presented here. Furthermore, they could not find a 
significant change in contour lines for the different percentage-seawater mixing values between high and low tide, which is, apart from the mixing zone, also observed from the Waikanae monitoring data in Fig. 5.8. Modelling of the influences of different tidal ranges suggested that the effects of tidal activity are stronger near the water table and less pronounced at the bottom of the aquifer, but that even small tidal ranges can have a significant influence on seawater intrusion (Ataie-Ashtiani et al., 1999). This may be an interesting implication for the shorter but more frequently acquired tidal DC resistivity monitoring time series from Te Horo, which, in Appendix C, are analysed in both a tidal and additional seasonal time frame to further gain an understanding of the importance of tides for seawater intrusion into a shallow coastal aquifer.

\subsubsection{Warping costs and cycle times - or: Is the resistivity signal depen- dent on the tides?}

The resistivity ratios and time series plots already show to some extent which parts of the aquifer undergo similar changes with time, and might therefore have similar hydraulic properties. However, it is not a trivial task to understand potential lag times related to tidal stage changes from these images as was additionally shown in Fig. 5.8. Hence, alternative analyses of the geoelectrical monitoring data were sought to improve the understanding of the role of diurnal tidal cycles on saline intrusion into shallow coastal aquifers.

Tidal stage and resistivity time series both show a sinusoidal temporal behaviour, which therefore leads to the impression that tidal processes may influence the resistivity changes on a small time scale (i.e. hours) within the near-shore part of a coastal aquifer. As is apparent from Figs. 5.3 and 5.7, most of the resistivity time series have longer periods than the approximately 12 hours of one tidal cycle. For this reason the method of dynamic time warping (DTW) was used to align the two signals in time and find the resulting distance (i.e. their similarity). This method is explained in Section 3.5, but basically looks for a minimum cost path through a recursively computed cost matrix of Euclidean distances between each point in the time series. Data handling was outlined earlier in Section 5.2.3. Note that the optimal warping cost (OWC) found by DTW can technically not exceed the length of the time series (i.e. 21 hours).

In Fig. 5.9 the OWC between tidal stage and resistivity signal (a) and groundwater stage 
and resistivity signal (b) for each model cell of the 21-hour monitoring found through DTW of the two time series along the Waikanae N2 profile in November 2015 are illustrated. Blank inversion cells are either due to reliability issues in the resistivity models found by the DOI appraisal method, model cells that were not considered to contain any seawater (i.e. $\rho>140$ $\Omega \mathrm{m}$ ), or because the resistivity range within the monitoring time did not exceed $5 \Omega \mathrm{m}$. OWC can be understood as how similar the resistivity signal of the aquifer is compared to the tidal or groundwater stage signals, respectively, for the same time span. Blue colours can therefore be interpreted as model elements that only take a short time to show a resistivity change with time relative to the compared signal (i.e. tidal or groundwater stage), whereas those in the redder colours indicate longer adjustment times in response to a changed seawater-freshwater pressure relationship. As was discussed earlier, aquifer parts that are within the wave run up zone and close to the shore line are generally expected to have shorter OWC's with respect to the tidal signal than those further inland, simply due to closer proximity to the wave run up and therefore the shorter travel times of the seawater within the aquifer matrix and hence, dependency on tidal forcing. In Fig. 5.9 this part was totally blanked out however, because within the 21-hour monitoring period an absolute resistivity variation of $5 \Omega \mathrm{m}$ was not exceeded for these cells. These parts of the aquifer have continuously low resistivity values suggesting a relatively high saturation with seawater ( $>10 \%$ ), and therefore tidal fluctuations may only be of minor importance to resistivity changes in these areas anyway.

However, there are several areas within the aquifer that show mixed optimal warping costs of the resistivity signal with respect to the tidal or groundwater stages and hence, indicate a mixture of time dependency between the two respective signals across these model cells. Again, parts referred to as $A$ and $B$ in earlier discussions, which are areas of the subsurface that show an increase and decrease of resistivity with decreasing tidal stage, respectively, appear to roughly group together for the DTW analyses. Model elements attributed to category $B$ were previously interpreted as parts of the aquifer that must have longer reaction times to changes in tidal stage, which is also indicated by larger OWC values as shown in Fig. 5.9(a). In Fig. 5.9(b) where the resistivity signal was analysed in terms of groundwater stage, these parts $B$ show overall smaller warping costs, except for the model portion around 100 meters profile extent. The inverse relationship of DTW analyses of the resistivity signal with the tidal and the groundwater stage signals is also observed for parts marked $A$. It could be argued that where OWC's are 


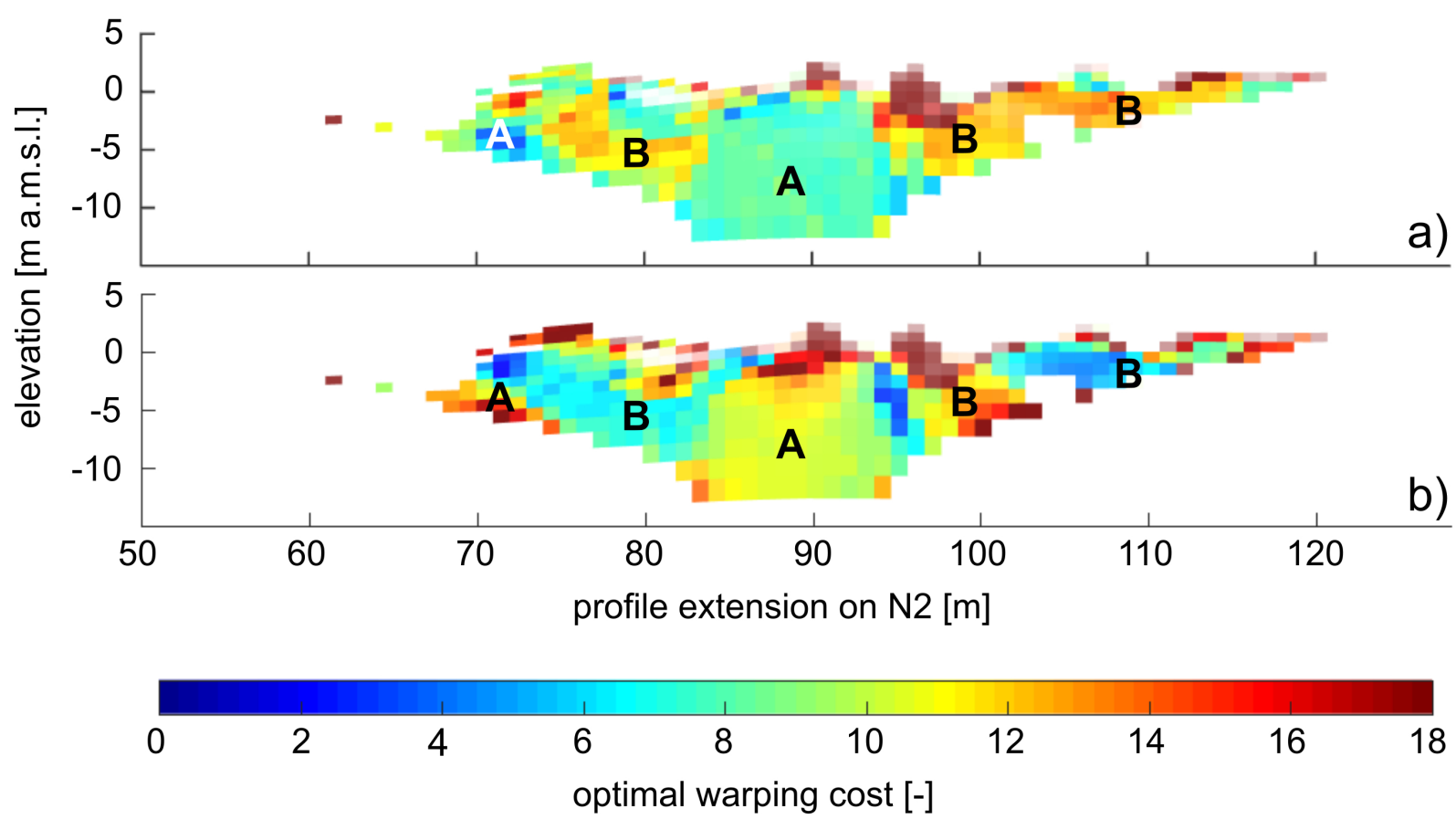

FIGURE 5.9: DTW between a) tidal stage and resistivity, and b) groundwater stage and resistivity signals of each individual model cell of the 21-hour tidal monitoring along the Waikanae N2 profile. Letters $A$ are associated with resistivity decrease during tidal stage increase in previous plots, whereas letters $B$ are marking parts of the aquifer showing a resistivity increase with increasing tidal stage.

smaller for a DTW analysis of model resistivity with tidal stage, the resistivity signal would be stronger influenced by tidal rather than groundwater forcing, and vice versa for model parts where OWC values are smaller for the correlation between resistivity and groundwater signals. Model parts with no dependency on either tidal or groundwater stage would presumably show a similar warping cost for each DTW analysis, as for example is the case for the model part termed $B$ around the 100 meter profile extent. Such areas could mark parts of the aquifer that show little change over time (i.e. stagnant water inclusions such as ancient seawater lenses). Other than simply the distance away from the coast, variation in hydraulic conductivity between these areas, where parts with a higher hydraulic conductivity show a smaller OWC to tidal stage and an increase in resistivity with decreasing tidal stage $(A)$, provides a rationale for the different reaction times. It can be hypothesised that such areas are preferential flow paths for seawater intrusion into the shallow coastal aquifer, whereas those exhibiting a larger time lag may not be of immediate concern in terms of a sustainable groundwater resource assessment.

Although, DTW and resistivity ratio plots seem to agree, it has to be kept in mind that the time series represent only a short time window into a continuous temporal process. If, as 
indicated from the above time series representations in Fig. 5.6, the resistivity signals have longer periods than the tidal time series (i.e. $>12$ hours), then it is inevitable that the section captured by the 21-hour monitoring only illustrates one possible relationship between the tidal and resistivity time series as a result of their different oscillatory behaviour. The impact of this ambiguity on the interpretation of DTW results is explored in a modelling study in Appendix E. Both the length and position of the monitoring window relative to the tidal and resistivity signals is important in calculating the OWC and without additional data it is impossible to state from OWC values alone which of the two signals is leading the other one. This is a considerable limitation in using this method for time-lapse DC resistivity investigations. However, the presented study makes use of independent data sets such as bore log information presented in Chapter 2 and groundwater chemistry data discussed later in Chapter 6, for verifying the findings from the time series analyses, and can therefore constrain the DTW results.

Because the DTW analysis only offers a comparison of the curve shapes of two signals and their relative shift, no information about reoccurrence of the signals is given. It is beneficial to know periods of signals under investigation in order to find similarities thereof. This is discussed in the following few paragraphs. First, a fast Fourier transform (FFT) is used to investigate dominant periods from time series spanning seven days (20. - 27. November 2015) of the tidal signal measured at the Waikanae River mouth, groundwater levels from two wells in the Waikanae groundwater zone (R26/6287 and R26/6886, see Fig. 2.1), and the atmospheric pressure recorded at Moa Point in Wellington. The tidal stage and atmospheric pressure were measured on a one-hour interval and later interpolated for 15-minute intervals, whereas the groundwater level data were already measured on a 15-minute interval and did not need interpolating. All signals were de-trended, smoothed and reduced to zero mean before the FFT. Fig. 5.10 shows the time series of each signal and their from FFT derived periods.

The tidal stage (black) in Fig. 5.10 measured over seven days shows the expected oscillatory characteristics of a semi-diurnal signal and a clear dominant peak at around 12 hours in the FFT analysis. Wells R26/6287 and R26/6886 measure groundwater stage within a similar stratigraphic portion of the aquifer at around six meters below surface, but while R26/6287 is only about $300 \mathrm{~m}$ away from the shoreline, R26/6886 is located just over a kilometer away from the coast. The consequence of this is likely reflected in Fig. 5.10 where the light blue time series 
(R26/6886) clearly reflects less of a tidal dependency than the dark blue one (R26/6287). This is additionally reflected in the smaller peak at a period of around 12 hours for the groundwater stage in well R26/6886 as compared to well R26/6287. A more dominant period is found around 17-18 hours and then later between 21-27 hours for the groundwater stage measured further away form the shore. To a less pronounced extent these periods are also visible on the FFT analysis for the dark blue groundwater stage measured in R26/6287. While it can be argued that the period around 21-27 hours may reflect a diurnal signal with the maximum amplitude at around 24 hours, the shorter 17-18 hour period needs to originate from something else. FFT analysis of groundwater stage measurements in the Te Horo groundwater zone (as discussed in Appendix F) from a bore tapping into the gravelly part of the aquifer shows a peak period of 21 hours for two different time windows. Though also showing a period of 11 hours, the longer period is clearly dominant and therefore provides additional confidence for the likelihood of resistivity and groundwater signals having a longer period.
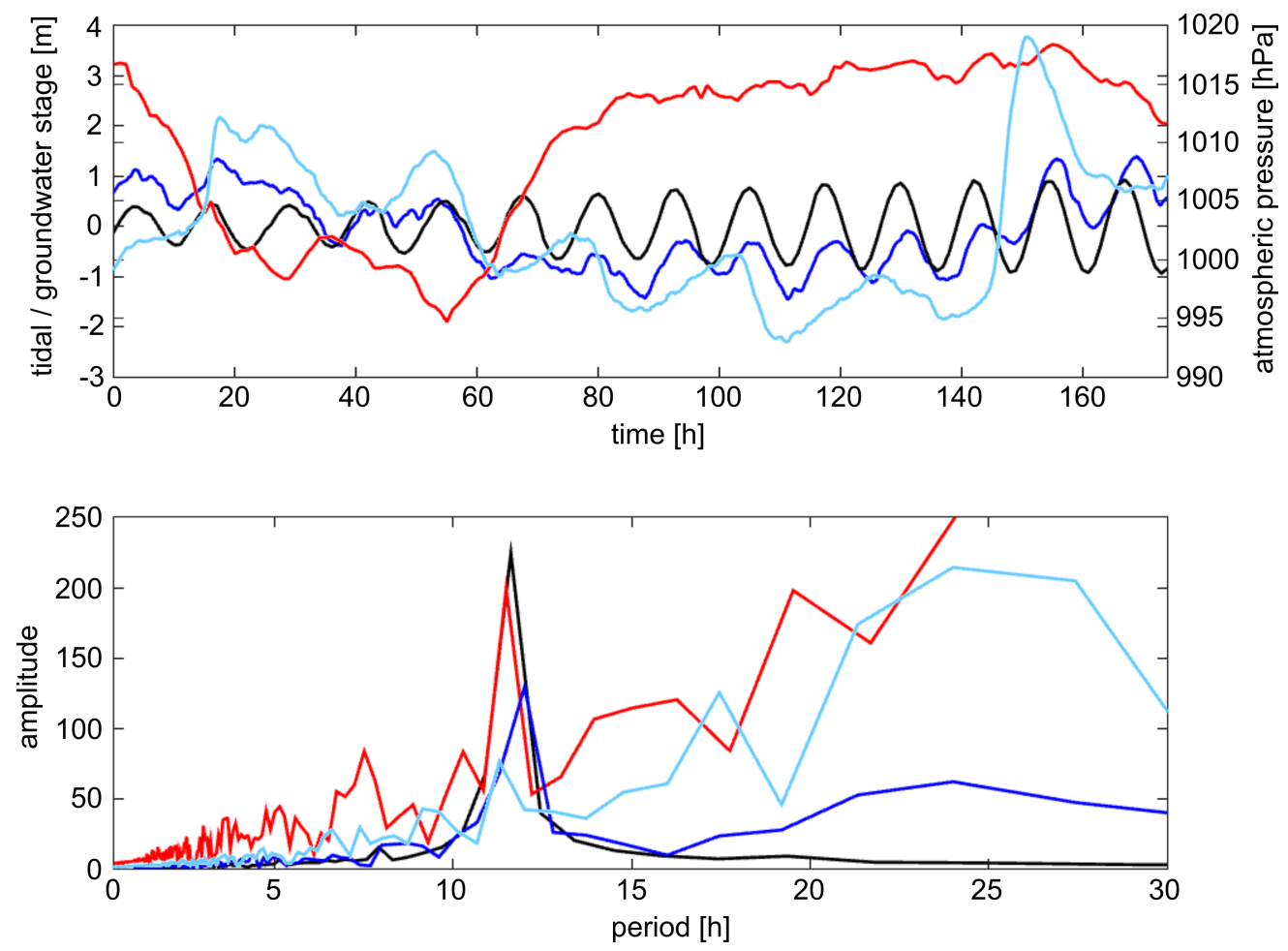

FIGURE 5.10: Seven-day time series and FFT derived periods of tidal stage at the Waikanae River mouth (black), groundwater levels in wells R26/6287 (dark blue) and R26/6886 (light blue), and atmospheric pressure at Moa Point in Wellington (red).

Groundwater stage is generally inversely proportional to atmospheric pressure. Comparing the time series of atmospheric pressure measured at Moa Point (red) in Fig. 5.10 with the 
two groundwater level measurements in blue colours, this can be observed to a certain extent. However, the atmospheric pressure data series has a very distinct amplitude around 12 hours after application of the FFT, which is not surprising considering that atmospheric pressure is dependent on temperature variations occurring in a semi-diurnal cycle similar to the one of the tidal signal. Although, the atmospheric pressure signal shows troughs where the groundwater signals show peaks, the clear dominance of a 12 hour peak at small periods implies that the groundwater periodicity does not depend on atmospheric pressure. Groundwater stage fluctuations depend preliminary on recharge and abstraction rates (= availability of freshwater). While the abstraction rate is hard to quantify with many unmetered bores taking groundwater at an unknown rate from the shallow aquifer, and the evaporation or evapotranspiration rates not being measured at the site, the river flow rate / stage can be used to obtain insight into recharge patterns as inherently this will be reflected in the shallow aquifer. However, the river stage is directly related to rainfall for which no period could be found due to its random occurrence pattern. It is therefore not surprising that the FFT analysis of the river flow at the Waikanae water treatment plant (Fig. 2.1) did not reveal a clear isolation of a dominant period. Furthermore, it has earlier been discussed that only aquifer parts in immediate vicinity to the river gravels have been evidenced to show direct relations to river flow. The reason for an 18 hour groundwater and resistivity period, hence remains unknown at this stage.

In order to find the period of the resistivity signal, the autocovariance analysis has been applied to each resistivity time series of the individual inversion model cells. The result of this is shown in Fig. 5.11, where elements that are fully blended (i.e. white), other than from previously stated reasons such as DOI indexing, are those for which no cycle time could be found using autocovariance. The reason for this is most probably because the time series are not long enough to detect a recurring extreme in these cases as their periods may be longer than the 21 hours of monitoring, or they were determined to be shorter than the 12 hours of the tidal cycle, which by assuming that the resistivity change is either following the tidal signal or dependent on a process with a larger period, is not a sensible value. The autocovariance of the tidal time series within the 21 hours of the tidal monitoring at the Waikanae field site in November 2015 gives an approximate 12-hour cycle time (see example in Fig. 3.6), which is the expected time it roughly takes between the same tidal extremes to re-occur in a regular diurnal tidal cycle (i.e. time between first and second high tide) and agrees with the period 
found from the FFT analysis of a seven-day record as shown in Fig. 5.10 (black signal).

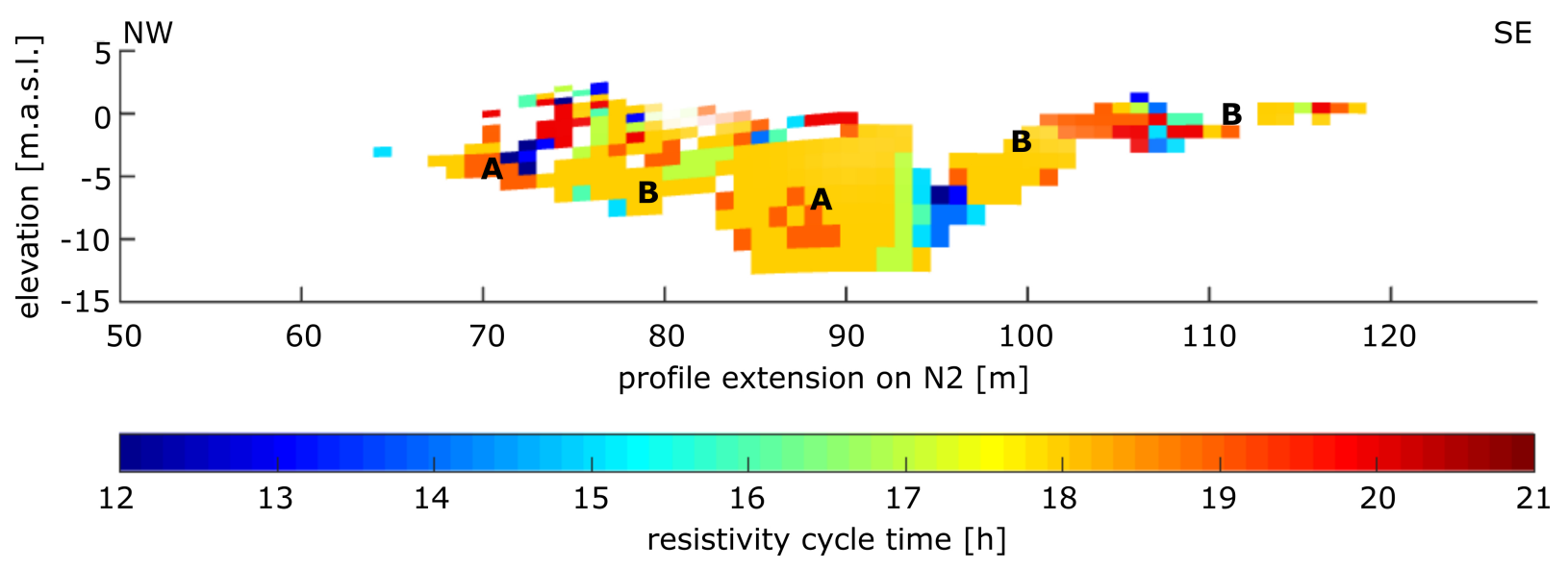

FIGURE 5.11: Autocovariance results of each model cell of the 21-hour tidal monitoring timelapse inversion along profile N2 in Waikanae. Model elements that exceed a period of 21 hours, are regarded not containing any seawater (i.e. resistivity $>140 \Omega \mathrm{m}$ ), have a resistivity range within the time series that is below $5 \Omega \mathrm{m}$ or show unreasonable cycle times, are blanked for interpretational reasons.

Apart from the wave run up zone between 50 and $70 \mathrm{~m}$ and some small parts within the aquifer, the resistivity periods found along Waikanae's profile N2 in Fig. 5.11 are in general around 17-20 hours, which is a good six hours longer than those of the tidal stage (i.e. 1.5 times the period of the tidal stage). This appears to be independent of parts previously marked $A$ and $B$, which means that resistivity variations in these two areas are always out of phase with each other. The average period found for the resistivity signal in each model cell implies that the main driving force for the resistivity changes in the aquifer is not mainly related to tidal forcing, but has a similar period to the one found from groundwater stage variations, where a similar period was especially dominant in wells further away from the coast where tidal influences are small. The system appears to be more likely dominated by groundwater pressure regardless of portions termed $A$ and $B$.

\subsection{Summary}

It is important to be able to determine preferential flow paths in a coastal aquifer system which are potential paths for seawater to intrude. In the feasibility study presented in this chapter it was aimed to gain an understanding of such dynamics from the results of a 24-hour DC 
resistivity monitoring along the N2 profile in the Waikanae groundwater zone.

By calculating resistivity ratios between inversion models of successive tidal extremes (i.e. high tide and low tide) and studying the behaviour of the resistivity time series, areas showing similar characteristics within a semi-diurnal tidal cycle were identified. Two main areas were evident within the aquifer models. These were termed $A$ and $B$ in order to be consistent with the previous chapter and indicate parts of the subsurface that show an increase and decrease in resistivity with decreasing tidal stage, respectively. Model parts identified as $A$ (showing an increase of resistivity with decreasing tidal stage) are considered to show a fast reaction to tidal stage changes and hence a smaller optimal warping cost between the resistivity and tidal signals, as indicated along the N2 profile section. Conversely, model resistivity time series indicating a decrease of resistivity with decreasing tidal stage (termed $B$ ) are expected to show larger OWC values for the same DTW analysis as compared to parts A. Again, this was conclusively shown along the N2 profile in the Waikanae groundwater zone. However, it was found from modelling (see Appendix E) that the choice of the monitoring window in both, length and position relative to the relationship of the tidal and resistivity signals, is crucial in order to obtain the observed termination of regions $A$ and $B$.

Analysing resistivity contour lines for three different seawater mixing ratios showed that the main changes over a diurnal tidal cycle occur in the part of the aquifer that marks most likely the mixing zone. Indicated groundwater flow paths from this inferred seawater dominance of the mixing dynamics during a rising tide, whereas the system appears to be more freshwater dominated when the tide is falling. However, these processes continue beyond the actual high and low tide times, respectively, which may be an indication of the processes being independent from tidal forcing. However, with the relatively short time span covered, this is impossible to quantify. The dynamics tie in with an inferred higher hydraulic conductivity in model parts marked $A$ and overall lower DTW warping costs between resistivity and tidal signals. These likely represent more gravelly parts of the aquifer as indicated from bore log information presented in Section 2.3.2. A higher hydraulic conductivity associated with gravels as compared to compacted sands, would also explain why these aquifer parts show higher percentage resistivity changes over time and hence appear to have higher seawater-freshwater mixing rates. 
From autocovariance analysis of the time series within the N2 resistivity model, periods of roughly 17 to 20 hours have been found for most of the aquifer (parts $A$ and $B$ ). Smaller periods of the resistivity signal were mainly found in the wave run up zone where the time series are highly fluctuating and therefore automatically lead to smaller cycle times between peaks in the signals. However, these model cells were not considered in the statistical analyses because their absolute resistivity change over the entire monitoring period was considered insignificant (i.e. $<5 \Omega \mathrm{m}$ ). These were also likely saturated with seawater to more than $10 \%$ at all times. Groundwater signal periods from two wells in the Waikanae groundwater zone have shown similar peak periodicities of around 17-18 hours. This was especially pronounced in the well further away from the coast where tidal influences can be assumed to be small. Considering the reasonably homogeneous distribution of resistivity signal periods, which are similar to the average groundwater period, it is likely that the aquifer is predominantly influenced by groundwater forcing, whereas tidal forcing is only of minor importance. The source of an 18 hour period however, remains unknown. 


\section{CHAPTER 6}

\section{Combining groundwater chemistry and DC resistivity for characterisation of seasonal seawater intrusion dynamics}

"Earth provides enough to satisfy every man's needs, but not every man's greed."

Mahatma Gandhi, leader of the Indian independence movement (1869 - 1948)

In this chapter I present the results and implications of a triannual combined groundwater chemistry and geoelectrical monitoring study conducted in the Te Horo groundwater zone. I give a short overview of the chemical compositions of different waters in general, and the sampling procedures. Subsequent to this I extensively discuss different relationships between the measured ions and groundwater parameters in order to find indications for potential seawater mixing, which is later discussed in conjunction with resistivity data collected on the same dates. Long-term implication for seawater intrusion in Te Horo Beach are wrapping up this chapter. A majority of this chapter has been submitted as a paper to the Journal of Hydrology: E. Sutter and M. Ingham. Combining groundwater chemistry and electrical resistivity tomography for detection of aquifer seawater intrusion potential. 


\begin{abstract}
Groundwater chemistry can be used to identify the dominant processes taking place within an aquifer. If repeated over time and in different seasons this can help build a deeper understanding of eventual changes in these processes. This was attempted during this study by repeating in situ multi-probe measurements of groundwater temperature, EC, TDS, salinity, DO and $\mathrm{pH}$, along with laboratory based ICP-MS analysis of water samples for major cations (Na, $\mathrm{Mg}, \mathrm{Ca}$ and $\mathrm{K}$ ) and several trace metals ( $\mathrm{Sr}, \mathrm{Li}, \mathrm{Fe}, \mathrm{Mn}, \mathrm{Ba}, \mathrm{Pb}, \mathrm{Cr}, \mathrm{Al}$ ). A total of 18 bores tapping into the shallow coastal aquifer in Te Horo Beach were sampled up to three times during a one-year period. For reference, seawater and freshwater from the adjacent stream were also sampled twice, once in summer and once in winter. The comparison of several measured entities indicated seawater intrusion within the first $300 \mathrm{~m}$ from the shoreline at aquifer depths between 0 and $5 \mathrm{~m}$ below mean sea level. In this layer the dominating processes were identified to be evaporation/crystallisation during summer time. Other parts of the shallow aquifer did not show any conclusive indications for seawater mixing and the chemical composition of the groundwater stored in these parts of the aquifer mainly depend on recharge and weathering patterns. The behavioural patterns of the seasonally determined controlling processes of the groundwater chemistry are a function of aquifer depth and distance to the coast. Implications from the chemical groundwater monitoring matches results from the DC resistivity monitoring and continuous groundwater logging. Due to the immobile nature of bores used to access the groundwater, studies like this are always restricted to be valid over only a small geographical region. However, the results from the hydrochemical monitoring could be tied to results from simultaneously obtained geoelectrical models, thereby increasing the reliability of the resistivity models and at the same time allowing to extrapolate in situ measurements from the hydrochemical monitoring with greater confidence. A combination of these methods was also used in an earlier study at the field site and the comparison to the present investigations strongly indicates anthropogenically driven seawater intrusion has increased in the shallow aquifer during the past 10 or so years.
\end{abstract}




\subsection{Introduction}

The communities along the Kapiti Coast in New Zealand cover their demands for freshwater from a mixture of surface-, tank- and groundwater. Freshwater use is metered in some areas (e.g. Waikanae) and the local council attempts to conserve freshwater resources with this and other measures (i.e. repairing water pipeline leaks, restricting water take during dry summer months). Although this is creditable in terms of environmental friendliness, it also points towards a globally increasing problem - the one of a potentially decreasing quantity of freshwater resources. For a sustainable freshwater resource management plan, it is also important, apart from estimating the available freshwater quantity and its potential to renew, to determine the water quality. This essentially means regularly monitoring the freshwater chemistry. In terms of groundwater, the freshwater source investigated in this thesis, the first studies of nitrate monitoring within the Kapit Coast groundwater zones were in the early 1980's (Tidswell, 2009). In general, most of the groundwater studies have focused on nitrate-nitrogen and sulphate contamination as these are regarded to be the main factors in groundwater quality at this location (Jones and Baker, 2005). The source of these contaminants has been identified to be from horticultural and agricultural land use and the animal and sewage discharges involved (Jones and Baker, 2005; Tidswell, 2009). In a hydrochemical study carried out at a location further east from the Kapiti Coast a possible seasonal connection between groundwater chemistry variation and groundwater volume used for irrigation has been suggested (Jones and Baker, 2005).

In 1998 Te Horo Beach residents complained about the groundwater quality, reporting poor taste and odour, and high turbidity. This led to a local groundwater investigation through the council (Tidswell, 2009). Hughes (1998) found only small amounts of nitrate and faecal contamination from septic tanks that are widely installed in the settlement, but recommended not to use the groundwater for potable supply due to possible migration of the contaminants within the shallow aquifer. In addition, localised effects of saline intrusion in the form of elevated major ion concentrations were inferred at some of the beach front properties (Hughes, 1998). This led to the conclusion that the saline interface was located close to the coast as a consequence of a low aquifer throughflow in Te Horo Beach. A similar conclusion was drawn by Wilson (2003) who estimated a saline interface shift by at least 10 meters landwards along the Te 
Horo Beach settlement compared to its natural position. This was determined using a mixture of hydrochemical and geoelectrical observations. Comparison of groundwater conductivity in several bores throughout the township between the Hughes (1998) and Wilson (2003) studies suggests that the effects of saline intrusion have decreased. A statement that was also made by Jones and Baker (2005) in their regional groundwater report as a conclusion from decreasing three-monthly measurements of boron and zinc in a shallow bore in Te Horo Beach (R25/5164) since 1998. The follow-up report, published seven years later (Tidswell et al., 2012), no longer mentioned saline intrusion, implying that this was no longer a problem at the site.

Many studies have used chemical composition and stable isotope hydrochemical analysis of groundwater samples to indicate seawater intrusion (SI) into coastal aquifers (e.g. Mandal et al., 2011; Raj and Shaji, 2017). Although these techniques can be used to delineate a problem, they only represent stationary observations of a dynamic process that may change over time (i.e. with seasons) and it remains difficult to clearly pinpoint the driving force for contamination. Only a limited number of studies have used repeat measurements of such hydrochemical and stable isotope measurements of coastal groundwater in order to detect seasonal changes in aquifer chemistry (e.g. Wood and Harrington, 2015; Han et al., 2015). Both these studies were able to determine seasonal changes in groundwater chemistry and could relate them to specific processes, thus demonstrating the importance of having repeat measurements for determining the leading processes in groundwater contamination at at specific location.

Although groundwater sampling can lead to a broad understanding of the freshwater resource stored in an aquifer, in general this requires a dense network of bores. As this is not always available, some authors have used the DC resistivity technique in combination with hydrochemical analyses to map local seawater intrusion into groundwater (e.g. Ayolabi et al., 2013; Lagudu et al., 2013). These studies were able to successfully relate hydrochemical properties to respective geological layers in the subsurface and were therefore able to more confidentially extrapolate in situ measurements into a more regional domain. However, none of these studies was done repetitively. The data presented in this chapter are repeat measurements of both methods with the aim of identifying seasonal changes of SI into the coastal aquifer in the $\mathrm{Te}$ Horo groundwater zone and the dominant processes that drive this. To my knowledge this is currently the only study that presents such repeat jointly acquired sets of hydrochemical and electrical resistivity data. 
Although the Groundwater Quality State of Environment (GQSoE, former Groundwater State of Environment (GWSOE)) programme provides long-term (1994-2015) information about several hydrochemical parameters (e.g. total dissolve solids (TDS), nitrate-nitrogen, sulphate, iron, manganese, fluoride, lead, zinc, E. Coli), some of them were not measured during the entire monitoring period (i.e. major ions and metals since 2003) and the spatial coverage of shallow aquifer testing is rather sparse in some areas and hence might not be representative everywhere (Tidswell et al., 2012). Te Horo Beach has one of the largest number of private shallow bores per capita in the Wellington region, which allows for a dense sampling network. In conjunction with seasonal resistivity measurements the aim is to confirm seasonal saltwater intrusion patterns with the aid of groundwater chemistry analyses. The general assumption is that with decreasing freshwater pressure in summer, due to both increased groundwater abstraction for irrigation and other domestic use, and decreased aquifer recharge from precipitation, saltwater is able to intrude further inland than is the case in winter when freshwater pressure is generally higher, thus leading to an increase in TDS and seawater specific ions (i.e. boron, lithium, strontium, zinc) during summer time. However, results from the seasonal resistivity monitoring in Te Horo (Chapter 4) have shown that this scenario is not necessarily reflected by the resistivity measurements and alternative explanations may lead to a better match with observations in the field.

This chapter analyses the results from the groundwater sampling study in Te Horo Beach and investigates how the above expectations are met in terms of groundwater chemistry. Then the connection is investigated with regard to additional DC resistivity measurements repeated in the same interval as the hydrochemical sampling. First, a short discussion on the water composition of three relevant water types and information concerning the drinking water standard in New Zealand are given. Following this, sampling practice and analytical methods relevant for the hydrochemical data presented in this chapter are explained in Sections 3.3 and 3.4. Theory to understand the resistivity data collection and handling is provided in Sections 3.1 and 3.2. Sampling sites and field procedures are introduced together with information about specific data analysis for both methods within the course of this chapter. The hydrochemical monitoring results from the one-year period are then presented and their implications discussed. Continuous electrical conductivity (EC), groundwater stage and temperature monitoring data are then presented to establish a link between the hydrochemical and later presented DC 
resistivity results. At the end long term implications of seawater intrusion are discussed and a summary is provided to wrap up the most important findings.

\subsection{Chemical composition of different waters}

Not all water is equal. There exists a reasonably large difference in the composition and concentration of different types of water that results from variations in geological environment, weathering regimes, climatic conditions and biogeochemical processes (Ibanez et al., 2007). Therefore, it is not an easy task to establish a general rule for the composition of natural waters. In terms of this thesis three types of water are important - groundwater, seawater and surface water (i.e. river water). As only cations were analysed in this work, other species are not discussed here. There are four major cations - sodium $\left(\mathrm{Na}^{+}\right)$, magnesium $\left(\mathrm{Mg}^{2+}\right)$, calcium $\left(\mathrm{Ca}^{2+}\right)$ and potassium $\left(\mathrm{K}^{+}\right)$- that are abundant in all three types of water to a greater or lesser extent. In Table 6.1 an overview of the occurrence of these elements is given for seawater and river water together with hypothetical $1 \%$ seawater-river water mixing values for these elements. Groundwater is much more variable and highly depends on residence time (i.e. time of the water in contact with the rock matrix), flow rate (i.e. dilution and mixing time availability) and temperature (e.g. kinetic effects) (Ibanez et al., 2007). It is therefore rather meaningless to state an average groundwater composition.

Seawater has a very stable concentration in major cations (residence time of 2500-4000 years) and their relative proportions are approximately constant throughout the oceans (Ibanez et al., 2007). It has a high salinity that is mainly determined by its sodium-chloride ( $\mathrm{NaCl})$ content, but can contain considerable amounts $(>1 \mathrm{mg} / \mathrm{l})$ of trace elements such as strontium $\left(\mathrm{Sr}^{2+}\right)$ (Barlow, 2003; Hem, 1989). Riley and Tongudai (1964) discovered that the concentration of the trace metal lithium $\left(\mathrm{Li}^{+}\right)$is proportional to the chloride $\left(\mathrm{Cl}^{-}\right)$content in seawater. This is helpful in terms of ICP-MS measurements because chloride concentrations could not be measured with this method. Therefore, the lithium concentration can serve as an indication for chloride concentrations in a water sample. Seawater gets its elementary components from basically everywhere - rivers, glaciers, hydrothermal systems, and by wind and rain transport from land (Ibanez et al., 2007). With a pH of about 8.0 to 8.4 it is rather alkaline compared to river- and groundwater for which pH usually ranges between 6 and 8.5 (Ibanez et al., 2007). 
TABLE 6.1: Average composition and concentrations of major cations in seawater and river water. All concentrations are given in $\mathrm{mg} / \mathrm{l}$. Ratios between different cations can be used to identify the source of a water sample. The last column provides hypothetical values of a $1 \%$ mixing of seawater with river water which is considered to be enough to render freshwater undrinkable (Werner et al., 2013). Seawater intrusion should therefore be indicated by the changed ratios, especially those involving sodium.

\begin{tabular}{llll}
\hline Constituent & $\begin{array}{l}\text { Seawater (after } \\
\text { Hem (1989)) }\end{array}$ & $\begin{array}{l}\text { River water (after } \\
\text { Semkin et al. (1994)) }\end{array}$ & $\begin{array}{l}1 \% \text { seawater mixing } \\
\text { with river water }\end{array}$ \\
\hline Sodium $\left(\mathrm{Na}^{+}\right)$ & $10^{\prime} 500$ & 6.3 & 111 \\
Magnesium $\left(\mathrm{Mg}^{2+}\right)$ & 1350 & 4.1 & 18 \\
Calcium $\left(\mathrm{Ca}^{2+}\right)$ & 410 & 15 & 19 \\
Potassium $\left(\mathrm{K}^{+}\right)$ & 390 & 2.3 & 6.2 \\
TDS & $\sim 35 ' 000$ & $\begin{array}{l}<1000 \text { (Barlow, 2003), } \\
\text { due to suitability for human }\end{array}$ & $\leq 1340$ \\
& & consumption & \\
& & & 0.9 \\
$\mathrm{Mg}^{2+} / \mathrm{Ca}^{2+}$ & 3.3 & 0.3 & 17.9 \\
$\mathrm{Na}^{+} / \mathrm{K}^{+}$ & 26.9 & 2.7 & 5.9 \\
$\mathrm{Na}^{+} / \mathrm{Ca}^{2+}$ & 25.6 & 0.4 & \\
\hline
\end{tabular}

The composition of river water depends on which processes are dominant in its environment. For example, if weathering prevails the moderate TDS concentration will mainly be determined by the presence of $\mathrm{Ca}^{2+}$, whereas if rainfall is the leading process then TDS values will be low and $\mathrm{Na}^{+}$and $\mathrm{Mg}^{2+}$, will be more abundant relative to other major cations (Ibanez et al., 2007). Evaporative processes can increase the ionic content of surface water. For the majority of rivers the predominance in major cations is defined as $\mathrm{Ca}^{2+}>\mathrm{Na}^{+}>\mathrm{Mg}^{2+}>\mathrm{K}^{+}$. Groundwater is in many ways similar to river water but has usually higher amounts of TDS and also contains higher concentrations of trace elements and metals, such as iron $\left(\mathrm{Fe}^{3+}\right)$, as a result of the normally much longer turnover times occurring in aquifers (1000-10'000 years as compared to 16-20 days for rivers (Ibanez et al., 2007)) that allows the water to be in longer contact with the rock matrix and chemically react with it.

Most countries have drinking water standards that state the limits for several water constituents in order for the water to be considered safe for drinking. The Drinking Water Standards for New Zealand (DWSNZ) provides a framework for maximum acceptable values (MAV) of chemical determinants in New Zealand (Ministry of Health New Zealand, 2008) and was last revised in 2008. This value is considered to be innoxious to health if consumed over a lifespan of about 70 years. However, parameters measured in this thesis are generally of no health concern and 
TABLE 6.2: Measured parameters in this thesis and their guideline values as stated in Ministry of Health New Zealand (2008) (black) or WHO (2011) (blue) if there was no statement made in the DWSNZ. The last column states the main reasons for measuring these particular parameters. This will be discussed in more detail in Section 6.4 however.

\begin{tabular}{|c|c|c|}
\hline Measured parameter & Guiedeline value (GV) & Reason for measurement \\
\hline Temperature & Preferably cool, but no GV given & \\
\hline Dissolved oxygen (DO) & no GV, naturally $<4$ mg/l & Determination of seasonal variations \\
\hline $\mathrm{pH}$ & $7.0-8.5(5.5-9.0)$ & \\
\hline Electrical conductivity (EC) & $50-1500 \mu \mathrm{S} / \mathrm{cm}$ & \multirow{3}{*}{$\begin{array}{l}\text { Determination of seasonal variations, } \\
\text { seawater intrusion indication }\end{array}$} \\
\hline Total dissolved solids (TDS) & $1000 \mathrm{mg} / 1$ & \\
\hline Salinity & $0.5 \mathrm{ppt}$ & \\
\hline Sodium (Na) & $200 \mathrm{mg} / 1$ & \multirow{4}{*}{$\begin{array}{l}\text { Ratios of major elements to } \\
\text { determine origin of water }\end{array}$} \\
\hline Magnesium (Mg) & $200 \mathrm{mg} / 1$ (together with Ca) & \\
\hline Calcium (Ca) & $200 \mathrm{mg} / 1$ (together with $\mathrm{Mg}$ ) & \\
\hline Potassium (K) & $<2000 \mathrm{mg} / 1$ & \\
\hline Iron $(\mathrm{Fe})$ & $0.2 \mathrm{mg} / 1$ & \multirow{2}{*}{$\begin{array}{l}\text { Redox processes, groundwater } \\
\text { dominance }\end{array}$} \\
\hline Manganese (Mn) & $\begin{array}{l}0.4 \mathrm{mg} / 1(0.1 \mathrm{mg} / 1 \text { taste } \\
\text { threshold })\end{array}$ & \\
\hline Lithium (Li) & $0.7 \mathrm{mg} / 1$ & \multirow{3}{*}{$\begin{array}{l}\text { Seawater dominance indication, Li as } \\
\text { a proxy for chloride, Sr also } \\
\text { indication of residence time }\end{array}$} \\
\hline Strontium (Sr) & $4 \mathrm{mg} / 1$ & \\
\hline Zinc (Zn) & $1.5 \mathrm{mg} / 1$ & \\
\hline Aluminium (Al) & $0.1 \mathrm{mg} / 1$ & \multirow{5}{*}{$\begin{array}{l}\text { Can decrease resistivity }->\text { misleading } \\
\text { interpretation for seawater intrusion }\end{array}$} \\
\hline Barium (Ba) & $0.7 \mathrm{mg} / 1$ & \\
\hline Chromium (Cr) & $0.05 \mathrm{mg} / 1$ & \\
\hline Copper $(\mathrm{Cu})$ & $1 \mathrm{mg} / 1$ & \\
\hline Lead $(\mathrm{Pb})$ & $0.01 \mathrm{mg} / 1$ & \\
\hline
\end{tabular}

only guideline values (GV) have been determined for them. Several constituents discussed in this thesis are not listed in the DWSNZ but have been given GV's by WHO (2011). A summary of all measured quantities, their GV's and the reason to measure them is given in Table 6.2. Water that exceeds these standards may still be used for industrial or irrigation purposes.

\subsection{Field procedures and data analysis}

Te Horo Beach offers a good opportunity to sample the shallow coastal aquifer through a vast number of private bores. In Fig. 6.1 and all bores used for groundwater sampling are indicated with dots. The extended TH2 DC resistivity transect $\left(\mathrm{TH} 2_{\text {ext }}\right)$ along which the resistivity measurements were made, is marked in the southern part of the township. Over the course of a year eleven bores have been sampled three times in total, with the geoelectrical profile measured either on the same days as the groundwater samplings, or closely around these dates. 
This was done twice in winter (June 2015 and August 2016) and once in summer (February 2016) (green dots). Four additional bores were only made available after the initial June 2015 analysis. These are marked with blue dots in Fig. 6.1 and their datasets comprise groundwater information from February and August 2016. Along with these four additional bores that provide seasonal hydrochemistry data, seawater and water from the Mangaone Stream were also sampled twice. Three of the bores could only be sampled once during the one-year period due to bore owners being away when the groundwater sampling took place and having not turned on their bore pump during that time (groundwater inaccessibility). Information obtained from these bores are used only in relation to the particular time in the year they were sampled in, and are marked red in Fig. 6.1. Between December 2015 and August 2016 it was possible to install a permanent EC, water level and temperature logger in an undisturbed (i.e. not pumped) bore the location of which is indicated in orange (R) (Fig. 6.1). Bore depths are not known exactly for every bore. Those that were unknown and show up neither in any local driller's notes nor in the GWRC data base can be assumed to be shallower than seven meters which is the maximum depth before a consent is required. A depth of five meters was assumed for these bores as an approximate average depth. A summary of bore depths is given in Table 6.3.

\subsubsection{Hydrochemical data}

Samples from bores were always taken using the same procedure. First, water was run from an outside tap directly connected to the bore (i.e. unfiltered groundwater) into a PVC bucket, which was rinsed first with the tap water in order to avoid contamination from a previous bore (Fig. 6.2(a)). In the bucket a previously rinsed multi-probe sensor (YSI ProPlus, USA) was submerged and continuously measured temperature, dissolved oxygen (DO), electrical conductivity (EC), total dissolved solids (TDS) and salinity of the flushing water. In order to make sure the water analysed is representative of the groundwater at the bore's screen depth, measurements were only recorded once the parameters had stabilised. This usually took between 5 and 15 minutes depending on the frequency of usage of the bore, and water pressure. The $\mathrm{pH}$ was then measured using an EcoTestr pH2 meter (Oakton Instruments, USA). A table summarising all results from the in situ water testing is given in Appendix I Tables I.1 and I.2. Finally, the groundwater sample was taken from the still running tap by rinsing a sterilised 50 


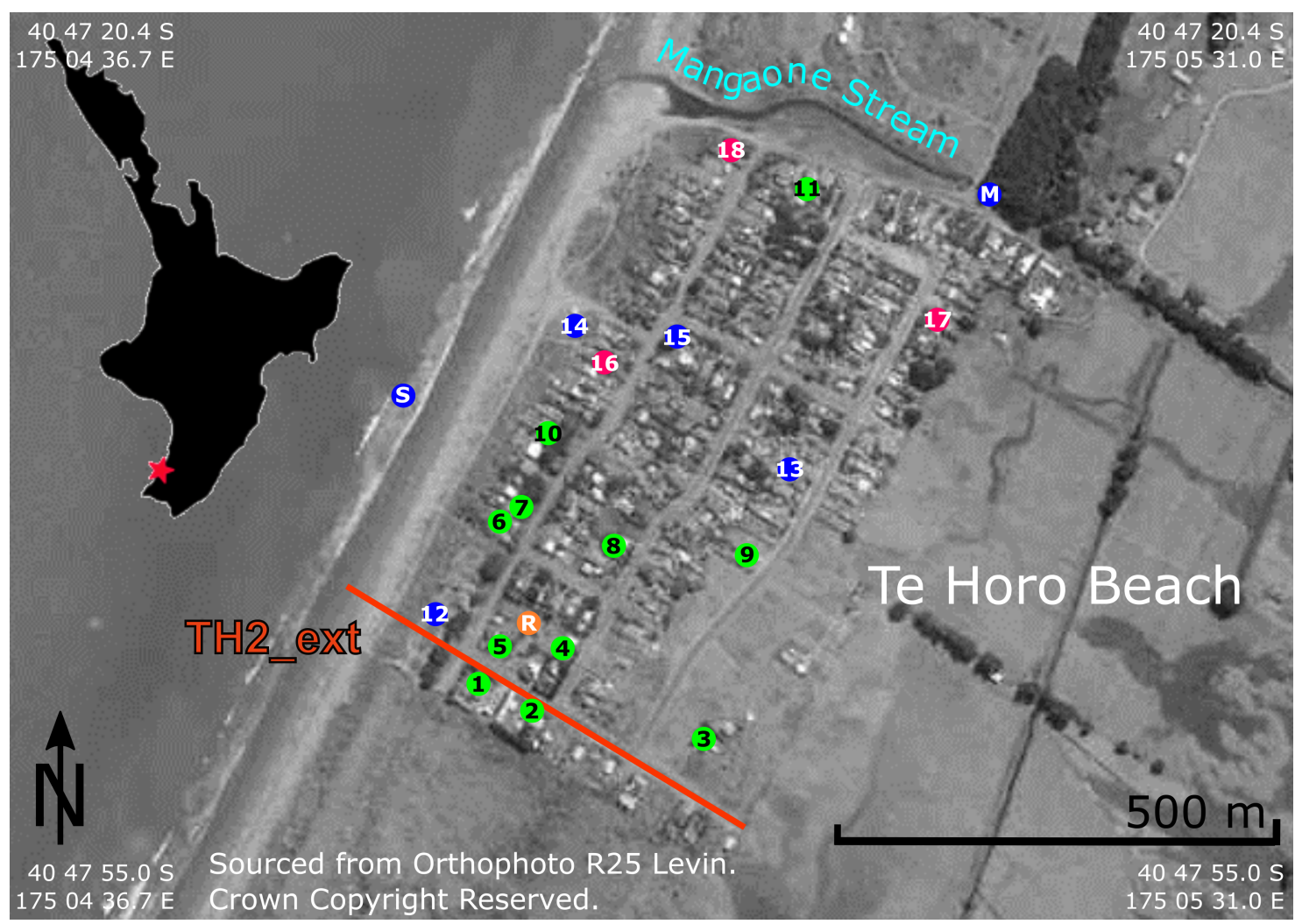

FIGURE 6.1: Map of the Te Horo Beach settlement with the Mangaone Stream to its north. Green dots indicate bore sampling locations where YSI sensor and ICP-MS hydrochemical data are available for all three sampling dates, while blue dots mark those where groundwater sampling results from February and August 2016 are available. Red dots indicate the location of bores that were only tested once during the sampling period. Long-term electrical conductivity (EC), groundwater stage and temperature monitoring was performed in the bore marked with the orange dot $(R)$. Geoelectrical monitoring was done along profile $\mathrm{TH} 2_{\text {ext }}$.

$\mathrm{ml}$ polypropylene (PP) tube (NEST Biotechnology, China) three times with the tap water before filling it up with the groundwater to just below the rim. The sample tube was then closed with its polyethylene (PE) cap and stored in a cooler equipped with cooling elements to keep the samples at low temperatures for transport. Because of long waiting times for the use of the ICP-MS instrument the samples had to be stored at about $4{ }^{\circ} \mathrm{C}$ in a fridge for up to 28 days. Sample holders were never opened during this time and because the parameters of interest are not of biological origin, sample deterioration should only be minor if at all. This could be confirmed by re-measuring two groundwater samples taken in February 2016 together with the ones taken in August 2016 (i.e. roughly 7 months after collection). Re-measurement showed an average change (increase or decrease) in the measured quantities of about $15 \%$ except for calcium which seemed to experience large changes during this time span (see Appendix I Table I.3). Although the re-measured February 2016 samples were stored constantly at around $4{ }^{\circ} \mathrm{C}$, they had been opened when the initial analysis was run on them in the beginning of March 
TABLE 6.3: Bore depth information for the 18 bores that were sampled for groundwater at least once during the one-year period (see Fig. 6.1 for locations). Coordinates are given in NZ Map Grid.

\begin{tabular}{llllll}
\hline Bore \# & Easting & Southing & $\begin{array}{l}\text { Elevation } \\
{[\mathrm{m} . \mathrm{a} . \mathrm{s} .1 .]}\end{array}$ & $\begin{array}{l}\text { Bore depth } \\
{[\mathrm{m}]}\end{array}$ & $\begin{array}{l}\text { Corrected } \\
\text { bore depth } \\
\text { [m.a.s.1.] }\end{array}$ \\
\hline 1 & 2685657 & 6043574.9 & 4.7 & 6 & -1.3 \\
2 & 2685725 & 6043553.3 & 5.1 & 8 & -2.9 \\
3 & 2685912 & 6043517.7 & 8.6 & 5 & 3.6 \\
4 & 2685753 & 6043613.4 & 5.1 & 5 & 0.1 \\
5 & 2685702 & 6043647 & 3.3 & 8.5 & -5.2 \\
6 & 2685673 & 6043767.4 & 5.5 & 5 & 0.5 \\
7 & 2685703 & 6043762.1 & 5.8 & 5 & 0.8 \\
8 & 2685800 & 6043722.4 & 6.6 & 5 & 1.6 \\
9 & 2685969 & 6043713.5 & 7.1 & 5.5 & 1.6 \\
10 & 2685717 & 6043861.1 & 6.6 & 6.5 & 0.1 \\
11 & 2686050 & 6044138.9 & 6.3 & 9 & -2.7 \\
12 & 2685608 & 6043665.9 & 5.8 & 21 & -15.2 \\
13 & 2685988 & 6043792.9 & 6.6 & 5 & 1.6 \\
14 & 2685770 & 6043995 & 2.8 & 5 & -2.2 \\
15 & 2685895 & 6043971.6 & 3.2 & 5 & -1.8 \\
16 & 2685812 & 6043961 & 4.9 & 5 & -0.1 \\
17 & 2686175 & 6044001.3 & 7.7 & 11 & -3.3 \\
18 & 2685934 & 6044185.1 & 1.9 & 21.5 & -19.6 \\
$\mathrm{R}$ & 2685720 & 6043654 & 3.2 & 3.5 & -0.3 \\
\hline
\end{tabular}

2016. Hence, the change in the measured concentration is possibly due to the introduction of fresh air when opening the sample containers, allowing for chemical reactions. However, the average calibration standard bias for the August 2016 measurements was $7 \%$ and $4 \%$ higher than for February 2016 analyses in major and trace elements, respectively, accounting for some of the observed change.

Before ICP-MS analysis $20 \mathrm{ml}$ of each groundwater sample taken in the field were filtered with a syringe driven $0.22 \mu \mathrm{m}$ ployethersulfone (PES) membrane filter (Jet Biofil, China) into acid washed and deionized water rinsed new sample tubes (Fig. 6.2(b)). This was in order to avoid any solid residues like sand or dust particles in the samples that could clog the intake to the ICP-MS instrument. The filtered samples were acidified with $0.3 \mathrm{ml}$ concentrated nitric acid in order to prevent chemical reactions of the open samples. To avoid clogging the instrument and to ensure comparable TDS in the introduced solutions, samples were diluted with $1 \%$ nitric acid according to their TDS contents. Dilution factors for major element (i.e. Na23, Mg24, 
Ca43 and K39) analysis varied between 200 and 1200 times, and for trace elements (i.e. Fe56, Mn55, Li7, Ba137, Pb208, Al27, Cr52, Cu63, Zn66 and Sr86) 2 to 12 times. Only cations were analysed due to difficulties in detecting anions with the ICP-MS system. Because seawater has much higher ion concentrations it had to be diluted at least 10'000 times to ensure the large amount of TDS was not a problem for the instrument. Each dilution was weighed (i.e. sample and total weight) (Fig. 6.2(c)) with an analytical balance (Ohaus, Australia) so that the effective dilution factor could be defined with much higher accuracy $( \pm 0.0001 \mathrm{~g})$.

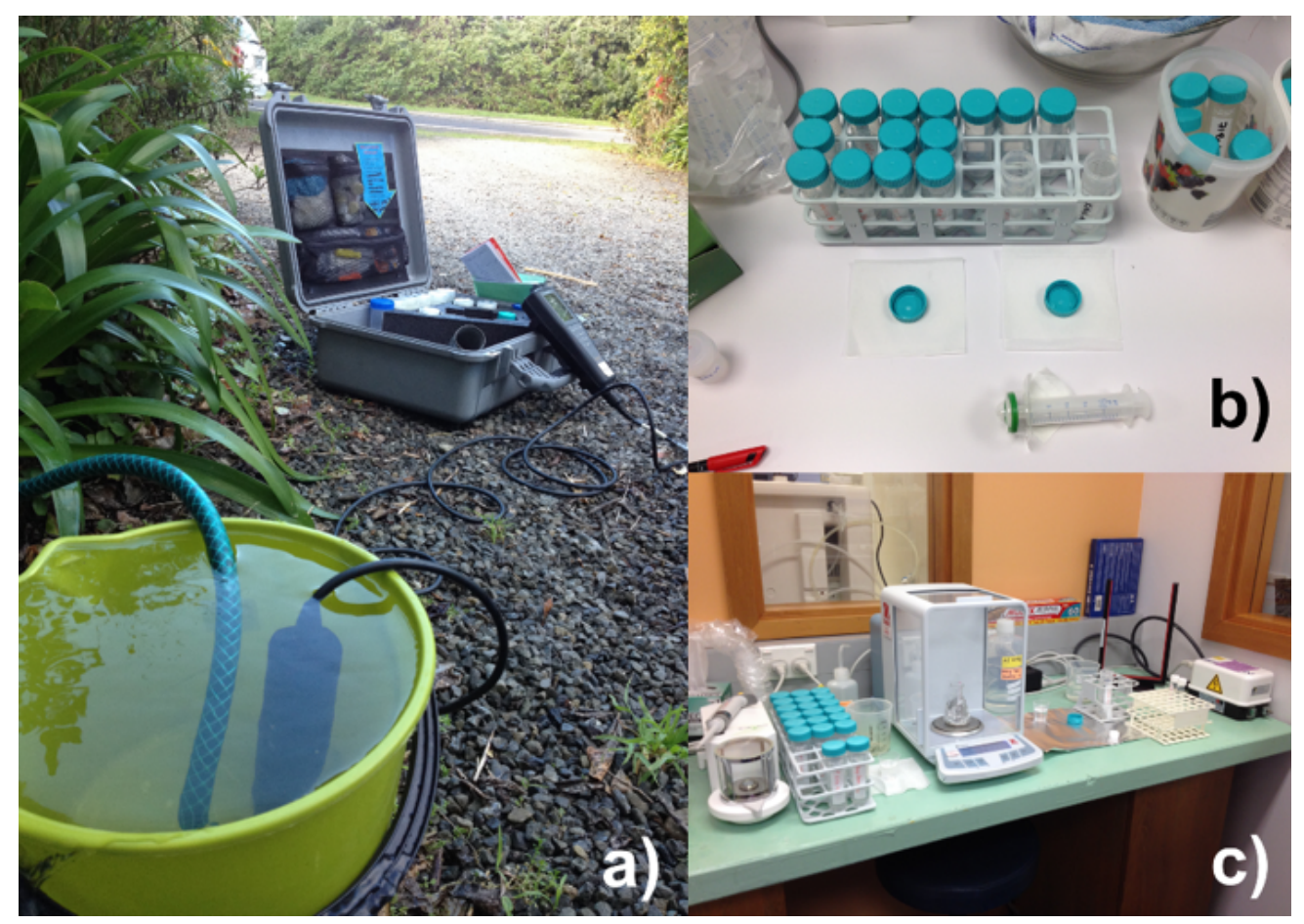

FIGURE 6.2: a) Groundwater sampling in the field. The multi-probe sensor in the bucket determines parameters like EC, TDS, salinity, temperature and dissolved oxygen of the continuously running groundwater. Once the parameters stabilize it can be assumed that the water is representative of the extraction depth and the groundwater sample can be taken. b) Sample filtering in the laboratory in order to remove solid particles before acidification process. c) Dilution of the samples with $1 \%$ nitric acid. Actual weights of the dilution are measured and noted in order to calculate an accurate dilution factor for concentration recovery after ICP-MS measurements.

Because the ICP-MS instrument (Element2 by Thermo Fisher Scientific, USA) measures the number of atoms of an element in a sample rather than a concentration, calibration standards were made from 1000 ppm ICP-MS single-element aqueous solutions (VHG Labs Inc., USA) containing all major and trace elements measured, respectively. This way the compositions were exactly known and allowed for calculation of each element's concentration in the groundwater samples using Eq. 3.29. Furthermore, river water certified reference material SLRS-4 (Yeghicheyan 
et al., 2001) was used as the secondary standard in order to estimate the accuracy of each measurement. This was done by calculating the bias for the secondary standard concentration using a similar form of Eq. 3.30. Only elements with a bias within $15 \%$ were considered trustworthy and therefore used for interpretation. The overall accuracy of each ICP-MS run was determined using the average bias between known and measured calibration standard concentrations for major and trace elements, respectively. This was favoured over the SLRS-4 bias because bias in the secondary standard for some of the elements gave rise to concern over their precision in order to be useful (e.g. low counts). The average calibration standard biases amount to $1.75 \%$ (Jun 15), $1.72 \%$ (Feb 16) and $9.07 \%$ (Aug 16) for major elements, and $1.95 \%$ (Jun 15), $3.21 \%$ (Feb 16) and 7.53 \% (Aug 16) for trace elements. With the February 2016 measurements, a filtered $1 \%$ nitric acid blank was also analysed in order to determine if there was any contamination of the samples from the sample tubes they were stored in or the filters used to eliminate solid particles from them. The element concentrations found from this filtered acid blank were in the order of $10^{-4}$ to $10^{-7} \mathrm{ppm}$. This is negligible and hence sample contamination from storage or filter equipment can be excluded.

\subsubsection{Geoelectrical data}

In order to have resistivity data for comparison with the hydrochemical data, an extended profile of the Te Horo profile TH2 (named TH2 $2_{\text {ext }}$ ) was, with exception of the first sampling which commenced about two months earlier, collected on or close to the dates the groundwater samplings were conducted (08.05.2015, 22.02.2015 and 22.08.2016). The profile extended along Kitchener Street (Fig. 6.1) over a total length of $495 \mathrm{~m}$ and was acquired with a 5-m minimum spacing and a Wenner- $\alpha$ array. This was achieved with the roll-along technique using a total of five cables with eight channels each. The time of measurement of the entire transect was in the order of three hours, started to the eastern side of the settlement and ended at the shore line. On all three occasions every electrode has been watered with saline water to ensure good ground contact and a sufficiently low $(<1500 \Omega \mathrm{m})$ electrode resistance throughout the data acquisition. Data handling and time-lapse inversion were carried out in the same manner as all other 2D time-lapse inversions presented in this thesis and a more detailed discussion of this can be found in Chapters 3 and 4. Electrode recovery for each of the repeat measurements was again done using fixed landmarks, the 5-m takeouts of the cables, and GPS positioning of 
every eighth electrode. Differential GPS topography information was also incorporated into the inversion process. Roughly $15 \%$ of all data were deleted in the editing process. These were mainly due to bad ground contact when crossing roads and driveways. Errors for all three measurements were estimated to be below $5 \%$ using a combination of repetition and reciprocal errors. The final resistivity models were obtained with an overall RMS error of $3.5 \%$ and model appraisal was done using the statistical approach to determine the DOI cut-off factor as suggested by (Deceuster et al., 2014).

Seven of the total 18 sampled bores in Te Horo (1, 2, 3, 4, 5, 12 and $R$ in Fig. 6.1) are located reasonably close to this transect and their electrical conductivity (EC) measurements were therefore transformed into bulk resistivity $\left(\rho_{b}=F / \sigma_{f}\right.$ with $F$ being 2.75 (Wilson et al., 2006)) in order to be comparable with the resistivity models.

The combination of monitoring groundwater and geoelectrical transects together has the aim of investigating seasonal seawater intrusion patterns in the aquifer in terms of its hydrochemistry and resistivity changes over time. In Fig. 6.3 the groundwater stage and rainfall at the Te Hapua wetland, located close to the Te Horo Beach settlement (Fig. 2.1), are shown for the entire sampling period. At the time of the first groundwater sampling in June 2015, as would be expected during the rainy season (winter), the groundwater level was high. The same observation applies to the August 2016 sampling. In summer 2016 (February sampling) the timing of the investigations was somewhat unlucky in that it commenced after a significant rain event in the driest period of the year. However, the low precipitation during the two months prior to the sampling should still have provided conditions representative of the aquifer during the dry period as was inferred from seasonal DC resistivity data in Chapter 4.

\subsection{Hydrochemical monitoring results and their implications}

The results presented in the following sections are based on a combination of the YSI sensor measurements obtained directly at the field sites (i.e. in situ measurements) and the hydrochemical groundwater analyses performed in the laboratory using the ICP-MS method. All data used for producing the figures in the following sections are given in Appendix I. Different combinations of measured parameters are used to demonstrate relationships between seawater 

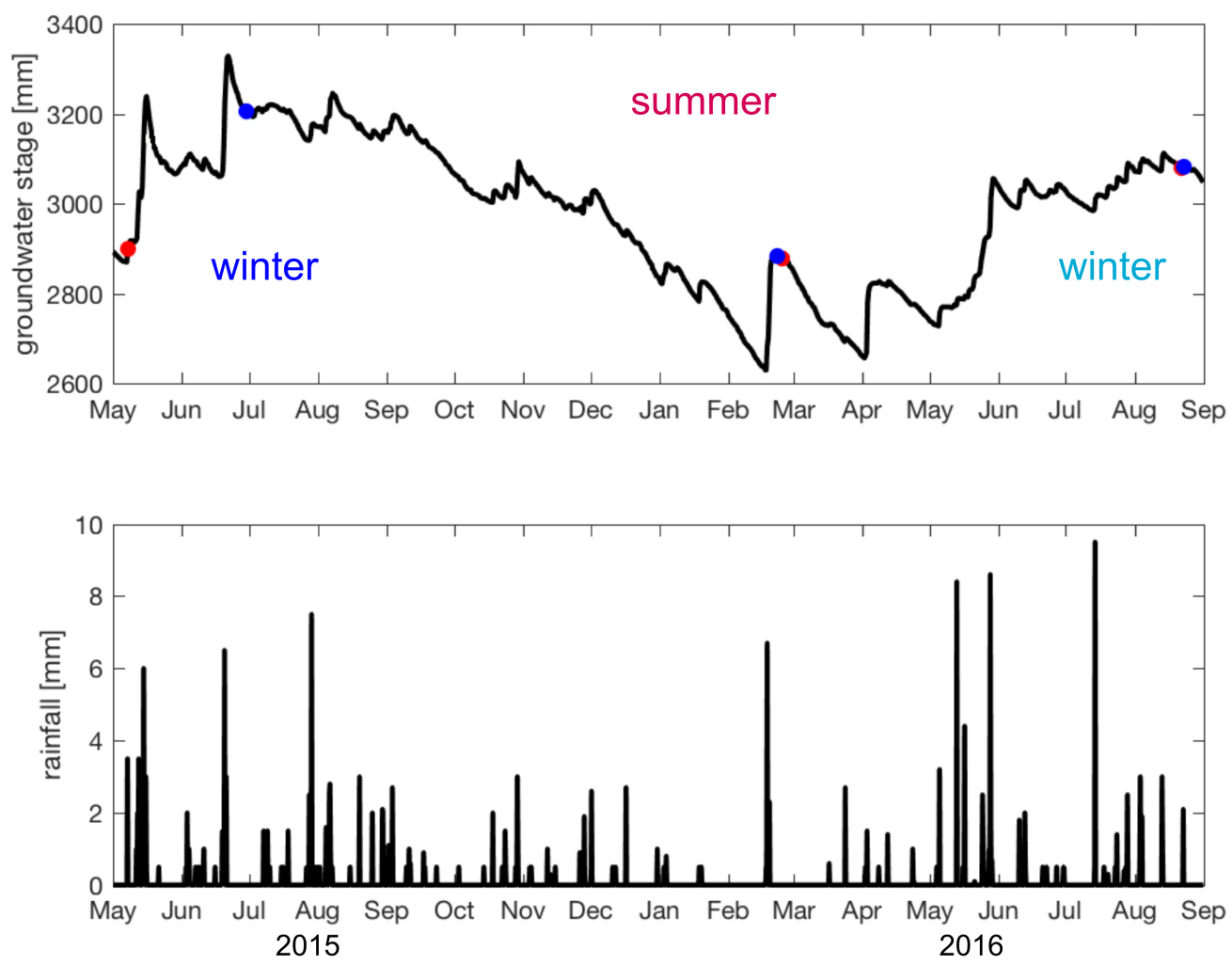

FIGURE 6.3: Groundwater stage (top) and rainfall (bottom) measured at the Te Hapua wetland GWRC environmental monitoring network station (location see Fig. 2.1) from May 2015 until September 2016. Blue dots indicate the times of the groundwater sampling, and red the ones of the resistivity measurement campaigns, respectively.

and freshwater dominated systems and to image observed seasonal variations in these. For analysis, only data that passed the criteria outlined in Section 6.3.1 (calibration standard bias < $10 \%$, SLRS-4 bias $<15 \%$, total ion counts > quantification limit (6 standard deviations (SD)) were taken into account. With these restrictions none of the measurements for $\mathrm{Cu}$ and $\mathrm{Zn}$ could be taken as they showed SLRS-4 biases much higher than $15 \%$ in all three runs. A reason for this might have been the relatively low ion counts for these elements during the ICP-MS analysis. In general, major elements were much more robust and all data passed the above criteria, whereas many of the trace elements were problematic to measure reliably. For example, $\mathrm{Pb}$ could only be obtained for some of the samples in June 2015 and August 2016. Except for two bores where slightly elevated $(<10 \mathrm{ppb})$ concentrations were found, the concentrations for lead were in the order of $10^{-2}$ to $10^{-1} \mathrm{ppb}$. It is therefore inferred that $\mathrm{Pb}$ only exists in spurious amounts in Te Horo Beach groundwater. Aluminium, although seemingly with higher concentrations, had a similar problem, and manganese concentrations could only be measured in the June 2015 samples due to large SLRS-4 biases and low ion counts in the other runs. 
In the following sections, all data available are used to find relationships between several groundwater parameters and across seasons. The data points are not specifically appointed to their bores in every plot, but rather are colour coded according to the time of the year they were sampled in - dark blue for June 2015 (winter), magenta for February 2016 (summer) and light blue for August 2016 (winter). The following discussion is structured using groundwater parameters measured in situ with the multi-probe sensor and starts with those related to salinity, followed by investigations involving the $\mathrm{pH}$ of the groundwater. Redox reactions that may have a controlling factor in the shallow aquifer make up the final part of this discussion.

\subsubsection{Electric conductivity, total dissolved solids and salinity relation- ships}

The groundwater electric conductivity (EC), total dissolved solids (TDS) and salinity are all interrelated parameters (i.e. TDS and salinity are effectively calculated from EC by the sensor) that reflect how well the water conducts an electric current. The higher the number of ions dissolved in the groundwater (TDS), the better conductor it will be, and a high concentration of ions is also generally related to high salinity, although, strictly speaking, this does not necessary mean that this is related to the intrusion of seawater. In Fig. 6.4 this is graphically shown by plotting the in situ measured TDS against EC and salinity, which illustrates the linear relationship between those parameters. As is expected, the water becomes electrically more conductive with an increasing content of ions in the water (i.e. increased salinity). In theory, temperature changes of more than 5-10 degrees Celsius can also lead to a significant change in TDS measurements (Nelson, 2002). In this study only one bore (8) exceeds a seasonal temperature change of 5-10 degrees Celsius. However, this is related to a TDS change of only about $50 \mathrm{mg} / \mathrm{l}$. Temperature dependent TDS changes are therefore considered negligible at the field site of Te Horo Beach. Figure 6.5 clearly illustrates this by showing the temperature changes against changes in TDS for all bores and between subsequent measurements.

In general, the highest values of TDS and EC are found for measurements made in summer 2016. This is expected due to a decreased amount of freshwater present in the aquifer and, hence, a higher concentration of minerals in the water. However, there are bores that exhibit a relatively high TDS content year-round (bore no. 3, 10, 12 and 13), and some that continuously 

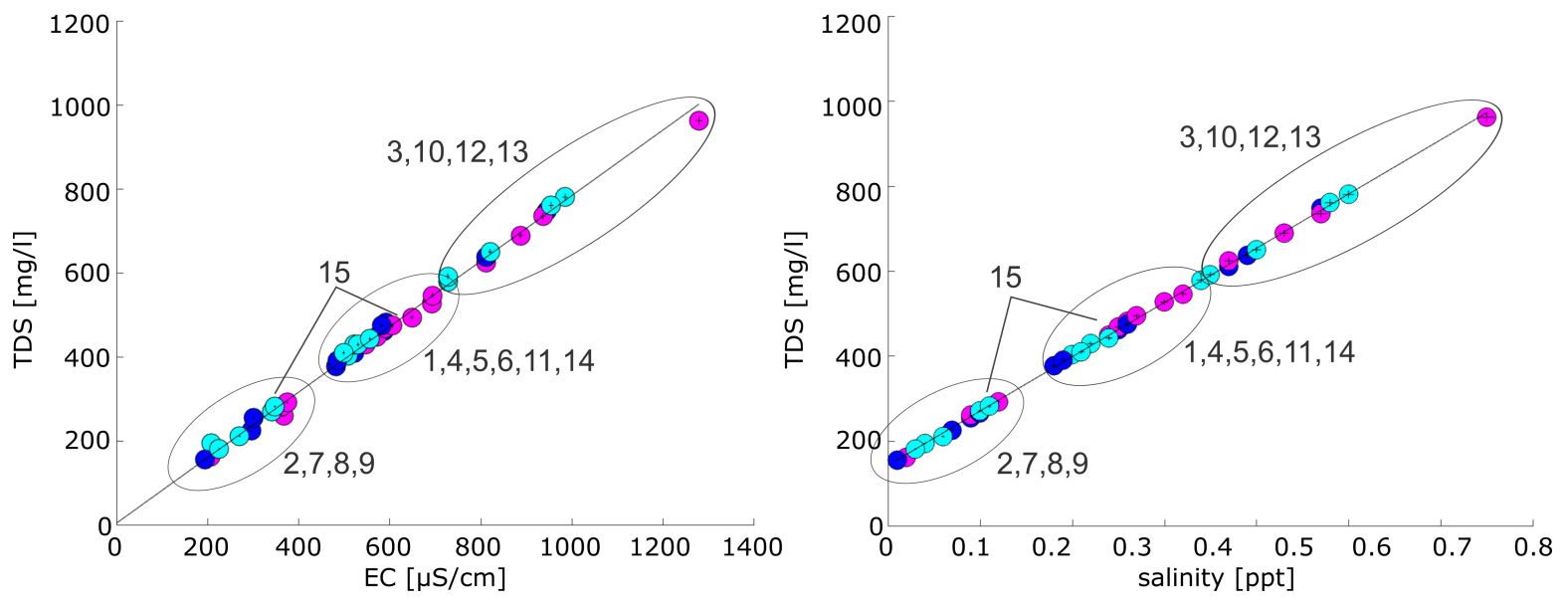

FIgURE 6.4: Total dissolved solids (TDS) vs. electrical conductivity (EC) (left), and TDS vs. salinity (right) measured in Te Horo Beach bores. Blue dots represent the two winter measurements, whereas pink ones are from the summer sampling.

show low readings (bore no. 2, 7, 8 and 9). Reasons for this are likely the varying depth of the boreholes (bores 7, 8 and 9 are more than $0.5 \mathrm{~m}$ above mean sea level) and a relatively heterogeneous geology in the shallow aquifer (i.e. the screen of bore 2 is at a depth of -2.9 m below sea level but shows some of the lowest salinity measurements in the study area). Low TDS, EC and salinity values are also found for the winter 2015 measurements in bore 17. With a depth well below mean sea level, the location of this bore a reasonable distance (i.e. $>400 \mathrm{~m}$ ) away from the coast may explain these values. Bores 3 and 13 show a high TDS content (high EC and salinity) although their depths are above mean sea level. As they are located approximately $500 \mathrm{~m}$ away from the coast they possibly tap into an ancient seawater inclusion or old stagnant groundwater pocket. The bores that are within about $300 \mathrm{~m}$ of the coast (no. 1, 4, 5, 6, 10, 11 and 14) and have screen depths close to or deeper than the mean sea level show electric conductivities between 400 and $800 \mu \mathrm{S} / \mathrm{cm}$ and TDS contents of 400 to $600 \mathrm{mg} / \mathrm{l}$ with a salinity lower than $0.5 \mathrm{ppt}$. Based on WHO (2011) guideline values this is still considered freshwater suitable to drink (although the taste may be unpleasant). Bores 16 (summer 2016) and 18 (winter 2015) both group into this category, too, although they are not plotted in Fig. 6.4. A summary of the three assigned main categories of bores based on their EC/TDS/salinity values is given in Table 6.4. There is one bore (no. 15) that belongs to category $L$ (August 2016) and $M$ (February 2016) depending on the season it was measured in.

In Fig. 6.6 all major elements measured with the ICP-MS method are plotted individually against their corresponding EC. A linear trend can be estimated for $\mathrm{Na}, \mathrm{Mg}$ and $\mathrm{Ca}$, and, with some exceptions, the same three categories as described in Fig. 6.4 and outlined in Table 6.4 can again 
TABLE 6.4: Bore categories according to their electrical conductivity, bore depth and bore characteristic. Bracketed bores were only measured once during the survey.

\begin{tabular}{|c|c|c|c|}
\hline Category & $\mathrm{EC}[\mu \mathrm{S} / \mathrm{cm}]$ & Bore description & $\begin{array}{l}\text { Bore numbers } \\
\text { associated }\end{array}$ \\
\hline $\mathrm{L}$ & $0-400$ & $\begin{array}{l}>0.5 \text { m.a.s.l. or distant from } \\
\text { coast }\end{array}$ & $\begin{array}{l}2,7,8,9,15 \text { (winter), } \\
(17)\end{array}$ \\
\hline M & $400-800$ & $\begin{array}{l}<0.5 \text { m.a.s.l. and within } 300 \mathrm{~m} \text { of } \\
\text { the coast }\end{array}$ & $\begin{array}{l}1,4,5,6,11,14,15 \\
\text { (summer), }(16,18)\end{array}$ \\
\hline $\mathrm{H}$ & $>800$ & $\begin{array}{l}<0.5 \text { m.a.s.l. and within } 200 \mathrm{~m} \text { of } \\
\text { the coast or tapping into ancient } \\
\text { seawater }\end{array}$ & $3,10,12,13$ \\
\hline
\end{tabular}

be distinguished. This is not surprising as the main part of the TDS amount in the groundwater samples will be attributed to the major elements. Bore number 4 shows medium concentrations of sodium but elevated concentrations of magnesium and calcium are found in the later winter sampling. Weathering from increased rainfall could account for an increased $\mathrm{Ca}$ and $\mathrm{Mg}$ release at this location in winter 2016. Another anomaly is the consistently low concentrations in all major cations for the June 2015 sample in bore 10 at a high TDS content. Since the data for the major elements measurement are of excellent quality (calibration standard bias $<2 \%$, SLRS-4 bias $<3 \%$, quantification limit (=6 SD) exceeded by a magnitude of $10^{6}$ ) it is likely that the data are representative and that the high TDS count at the low concentration in the major elements has another explanation. The sensor was always carefully rinsed with the new bore water before measurements were started, so that contamination of the multi-probe sensor from a previous bore can be ruled out as the source of ambiguous TDS measurements. Elevated nitrate-N or ammonia-N levels in the groundwater of the shallow coastal aquifer (Bekesi, 1996; Hughes, 1998) could lead to high TDS concentrations. These would be undetected in this study as they were not subject to investigation.

It is striking how consistent the concentrations in potassium are for all but two bores during the entire sampling period. The two odd bores (no. 3 and 13) are those pointed out earlier as possibly tapping into ancient seawater or old groundwater lenses as remnants from a prograding coast line (Tidswell, 2009). The long residence time of the groundwater in such lenses would explain the high amount of potassium (and all other major cations as well as TDS) as a consequence of a longer interaction with the rock matrix (e.g. feldspars) compared to all other bores that only show a low concentration in this element. In terms of seasonality it appears 


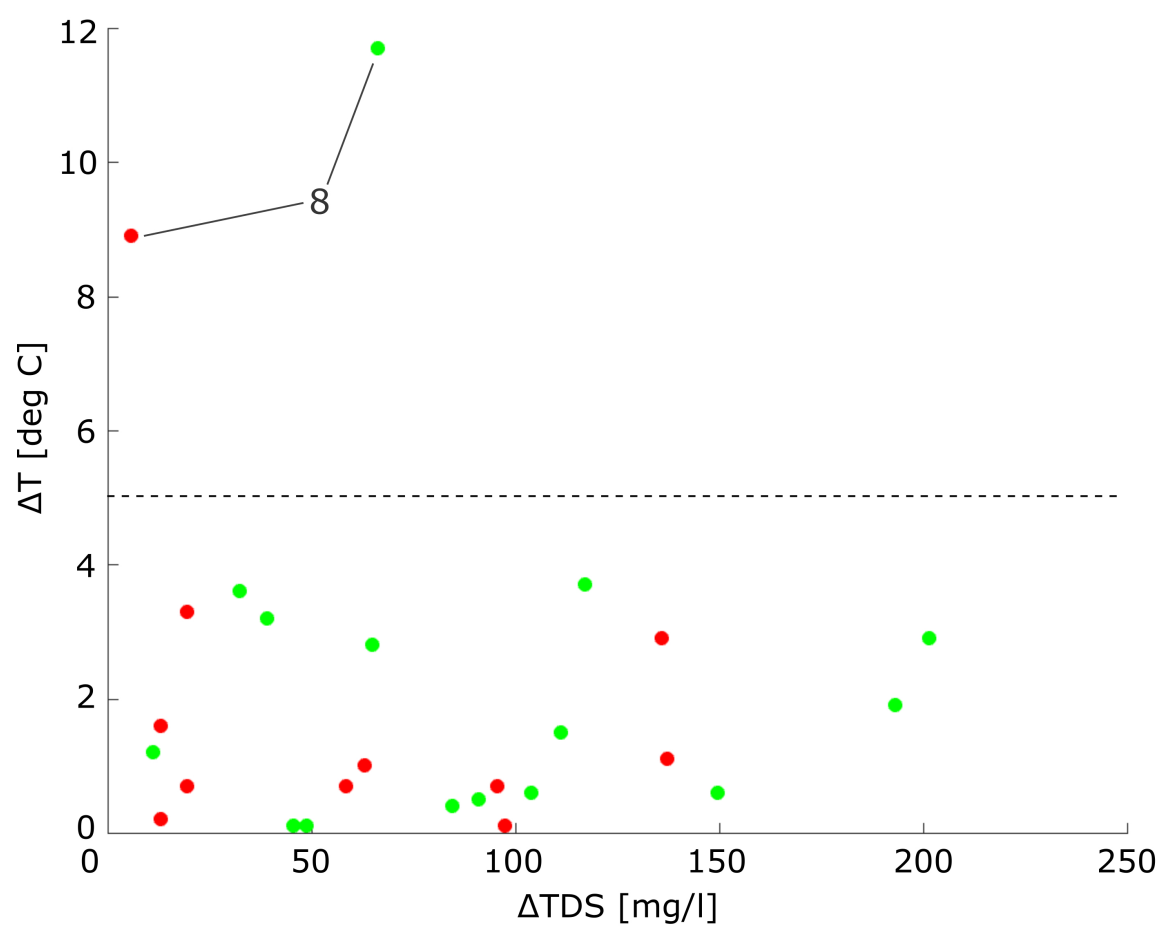

FIGURE 6.5: Temperature difference vs. TDS difference of all bore samples and between subsequent samplings (red dots $=$ abs(winter 2015-summer 2016), green dots $=$ abs(summer 2016-winter 2016)). The temperature variation is generally low between seasons and there is no notable trend visible with TDS. Only bore \# 8 exceeds a temperature difference of $5-10{ }^{\circ} \mathrm{C}$, which is considered the limit for temperature influence of TDS by Nelson (2002).

as if generally summer measurements (pink dots) show lower concentrations in Na, Ca and Mg compared to winter concentrations (blue dots) in these ions. Lower concentrations of elements in the water during summer time could mean that crystallisation/evapotranspiration is a dominant process leading to removal of these ions from the groundwater. This agrees with observations from the seasonal DC resistivity measurements discussed in Chapter 4.

One way of looking at the potential occurrence of seawater intrusion in an aquifer is by analysing the ratios found for the major elements (in this case only cations). There is a significant difference in these ratios between fresh- and seawater as shown in Table 6.1 for the average seawater and river water compositions on this planet. In Fig. 6.7 the ratios of $\mathrm{Na} / \mathrm{Ca}, \mathrm{Na} / \mathrm{K}$ and $\mathrm{Mg} / \mathrm{Ca}$ are plotted in relation to ratios found from measurements of the major cations in the seawater (S) at the sampling location, the Mangaone stream (M) north of the field site (as a representation of freshwater composition in the area), and a hypothetical $1 \%$ mixing of these two fluids. Looking at the ratio of $\mathrm{Na}$ and $\mathrm{Ca}$ it becomes evident that most bore samples lie above the line representing Mangaone stream water. This essentially means that their $\mathrm{Na} / \mathrm{Ca}$ ratio is 


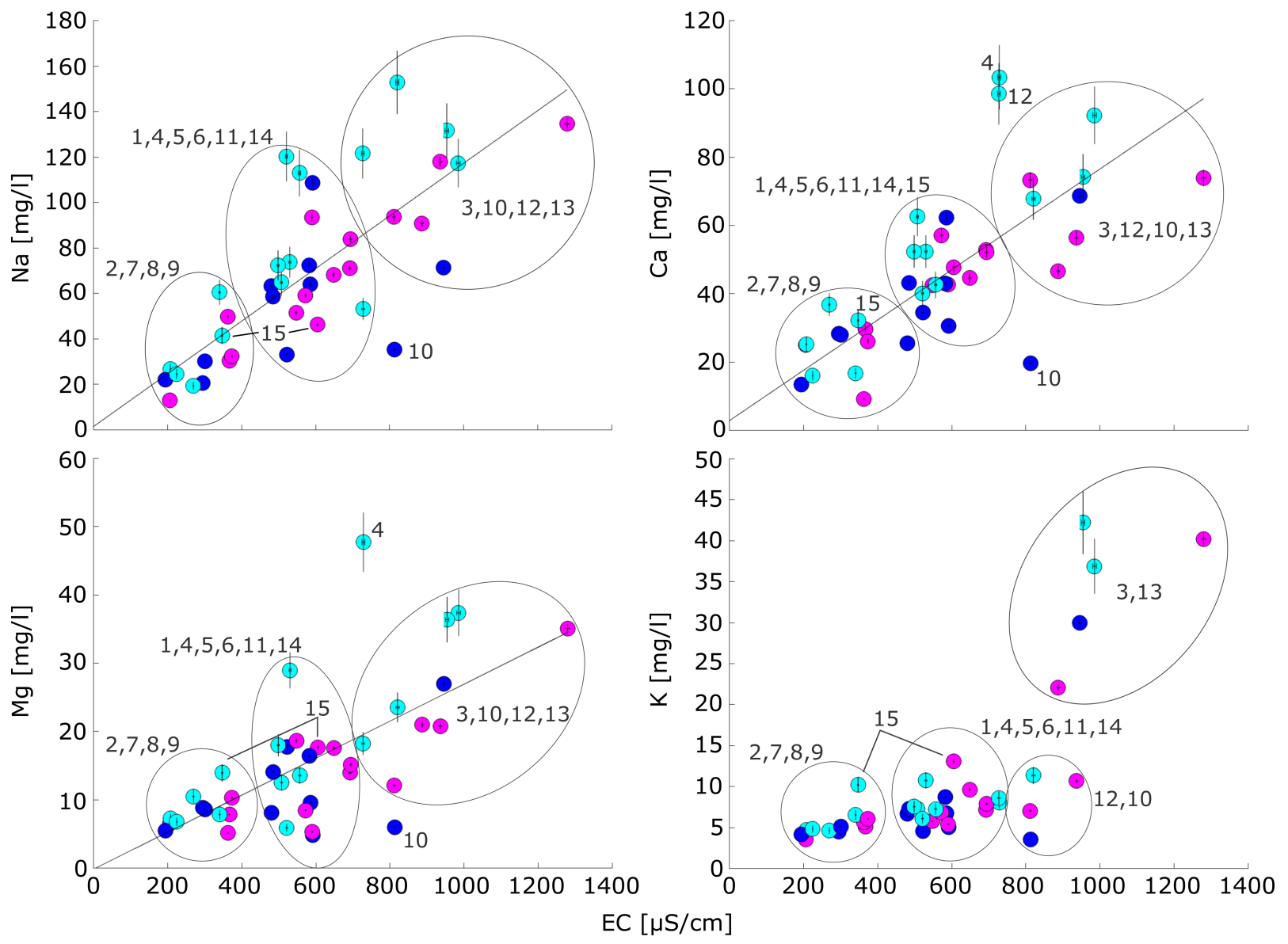

FIGURE 6.6: Electric conductivity plotted against the four major cations ( $\mathrm{Na}, \mathrm{Mg}$, $\mathrm{Ca}$ and $\mathrm{K}$ ). A positive linear trend can be found for $\mathrm{Na}, \mathrm{Mg}$ and $\mathrm{Ca}$, but is not evident in $\mathrm{K}$. Increasing concentrations in the major elements have a significant influence on EC as they account for the main TDS content in groundwater.

smaller than that of the freshwater stream, which can only be achieved if there is a depletion in sodium and/or an enrichment in calcium relative to the stream water. Custodio (1987) pointed out exchange reactions between sodium and calcium in a seawater-freshwater system that are related to adsorption processes. As freshwater is usually Ca-dominated (e.g. Table 6.1), for example as a consequence of the dissolution of feldspars, calcium becomes adsorbed by sediment grains in a freshwater system. If, however, seawater intrudes, the sediment surfaces have a preference to adsorb sodium ions instead and therefore calcium is released into the groundwater. This therefore leads to an increase in calcium and a decrease in sodium if seawater intrusion occurs, which is observed for most of the Na/Ca ratios in Fig. 6.7.

Seasonal variations are generally small (usually $<20 \mathrm{mg} / \mathrm{l}$ ) and differ, depending on the bore, between an increase of calcium in summer (e.g. 2, 11 and 15), an increase of calcium in winter (e.g. 4, 7, 9, 10 and 12) and relative stability throughout the year (e.g. 5 and 13). The bores 

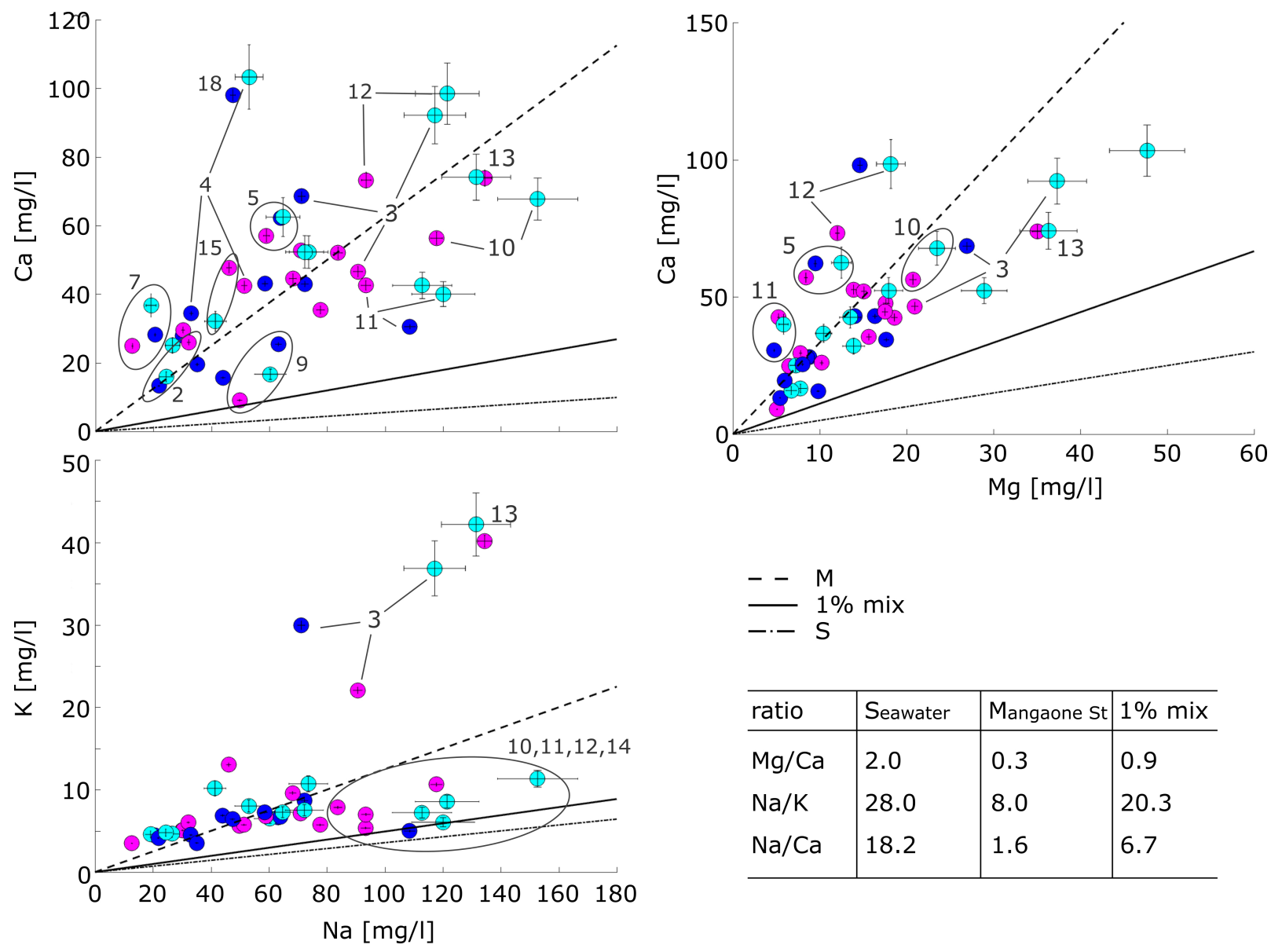

FIGURE 6.7: Major element ratios shown for $\mathrm{Na} / \mathrm{K}, \mathrm{Na} / \mathrm{Ca}$ and $\mathrm{Mg} / \mathrm{Ca}$. The plotted trend lines indicate the ratios of the relative major cations for average data measured in the Mangaone stream (M) representing freshwater, the seawater (S) west of the sampling site, and a theoretical $1 \%$ mixture of fresh- and seawater at this location (Fig. 6.1).

grouped according to their $\mathrm{Na} / \mathrm{Ca}$ behaviour with time did not all fall into the same categories ( $L, M$ or $H$ in Table 6.4) as in the previous discussion, which indicates that seawater intrusion does not necessarily need to be tied to high TDS content. However, when considering the screen depths, there is a commonality between them. Bores reaching depths between mean sea level and to about $5 \mathrm{~m}$ below mean sea level (bores 2, 11 and 15) show evidence for seawater intrusion from their increased Ca concentrations during the summer months. Bores that either extend only to depths above mean sea level (4, 7, 9 and 10) or tap into deeper parts of the aquifer (> $10 \mathrm{~m}$ below mean sea level - bores 12 and 18) indicate this process occurring in winter. Seawater intrusion occurrence in winter when groundwater levels and recharge are high is not intuitive, hence, the elevated Ca concentrations in the very shallow and the deeper parts of the aquifer during winter months may be due to an increased throughflow of freshwater that brings large amounts of calcium into the aquifer as opposed to Na-Ca exchange reactions 
due to intruding seawater. The above observations concerning bore depths and $\mathrm{Na} / \mathrm{Ca}$ ratios also match the resistivity structure and seasonal changes of Te Horo profile TH1 in Fig. 4.7. Similar conclusions can be drawn for $\mathrm{Mg} / \mathrm{Ca}$ ratios, though, with generally smaller variations observed in $\mathrm{Mg}$ as compared to $\mathrm{Na}$. Elevated calcium and magnesium concentrations can also be connected to high weathering rates induced from large precipitation rates for example. In this case the measured concentrations would be independent from sedimentary matrix reactions and the gradients of lines plotted for different samples in the same bore can give an indication of the hydrochemical composition of the groundwater. For example, bore 10 shows a gradient close to the one of freshwater, whereas the measurements of bore 5 appear to have a slightly less steep gradient towards a $1 \%$ seawater mixing trend. However, with only two to three measurements per bore and taking into account the errors of the individual data points, it is difficult to make meaningful statements from gradients.

Bores 3 and 13 in Fig. 6.7 show higher than usual potassium concentrations all year round, whereas all other bores have a relatively stable potassium content of less than $15 \mathrm{mg} / \mathrm{l}$. A high potassium concentration is generally thought to be a function of groundwater residence time and therefore supports the hypothesis that these two bores tap into groundwater that has not moved much in a long time (e.g. ancient seawater lens or stagnant old groundwater). The majority of the bores follows the trend of the fresh river water, but bores 10, 11, 12 and 14 seem to be closer to the trend line for a freshwater-seawater mixture, both value- and gradient-wise. Because $\mathrm{K}$ is not as reactive as for example $\mathrm{Na}$ and $\mathrm{Ca}$, it can be assumed that adsorption is not the dominant process for potassium concentrations in the groundwater. Hence, trends close to that of a $1 \%$ seawater mixture may indicate saline mixing at these locations. The four bores either belong to category $M$ or $H$ as defined in Table 6.4.

Apart from the major cations, a series of trace elements was also measured in the groundwater samples using the ICP-MS method. Due to reasons mentioned earlier the data for these elements were qualitatively not as good as those for the major elements. Concentrations in seawater could not be measured for trace elements and, hence, reference values in Table 6.5 are taken from Chester (2000). These are not plotted in Figs. 6.8 and 6.9 because the high values of salinity would constrict the abscissa in the plots.

Trace metal concentrations found in the groundwater samples in Te Horo Beach are generally 
TABLE 6.5: Trace element concentrations for average seawater at 35 ppt salinity as stated by Chester (2000).

\begin{tabular}{l|ll}
\hline Trace element & $\begin{array}{l}\text { Concentration }[\mathrm{nmol} / \mathrm{kg}] @ 35 \\
\text { ppt salinity }\end{array}$ & Concentration $[\mu \mathrm{g} / \mathrm{l}]$ \\
\hline Strontium (Sr) & $90^{\prime} 000$ & 8080 \\
Lithium (Li) & $25^{\prime} 000$ & 177.86 \\
Barium (Ba) & 100 & 14.08 \\
Aluminium (Al) & 20 & 0.55 \\
Chromium (Cr) & 4 & 0.21 \\
Iron (Fe) & 1 & 0.057 \\
Manganese (Mn) & 0.5 & 0.028 \\
Lead $(\mathrm{Pb})$ & 0.01 & 0.0021 \\
\hline
\end{tabular}

low for $\mathrm{Al}, \mathrm{Ba}, \mathrm{Cr}$ and $\mathrm{Pb}$ as illustrated in Fig. 6.8. Aluminium could only be detected in summer 2016 (more acidic water can contain higher Al concentrations (WHO, 2015) - see $\mathrm{pH}$ relationships in Section G.1), and there were detectable concentrations for chromium and lead only during the winter measurements. As chloride ions form an insoluble compound with lead (WHO, 2015), higher $\mathrm{Cl}$ concentrations in summer could explain the absence of $\mathrm{Pb}$ in the groundwater samples. Almost all bores show these $\mathrm{Al}$ and $\mathrm{Pb}$ occurrences in their samples. These trace metals were measured in order to detect possible influence on the DC resistivity measurements. However, none of the four trace metals analysed in Fig. 6.8 shows any clear relationship with EC. It is therefore unlikely that these elements have an influence on the DC resistivity measurements. Apart from Ba, the concentrations in seawater are less than $1 \mu \mathrm{g} / 1$, and it is assumed that trace elements $\mathrm{Al}, \mathrm{Cr}$ and $\mathrm{Pb}$ cannot be directly used as seawater tracers. Indeed, their measured concentrations are similar to or lower than those measured for the fresh river water sample $(\mathrm{M})$. This is probably a consequence of their low solubility in water. Barium can be used as a freshwater tracer (Yamamoto-Kawai et al., 2010) but typically shows values below $10 \mu \mathrm{g} / 1$ in the ICP-MS measurements. Two bores (3 and 13) show Ba concentrations higher than those measured in the Mangaone stream. These are the same two bores believed to be associated with isolated groundwater lenses and it is therefore possible that Ba accumulates over time due to low freshwater throughflow.

Strontium and lithium are two very abundant trace metals found in seawater (see Table 6.5) and are related to chlorine concentration (Riley and Tongudai, 1964; Barlow, 2003). A positive linear relationship with salinity (EC, TDS) of these elements in Fig. 6.9 can therefore be directly interpreted as an indication for increased seawater mixing. The elevated concentrations in $\mathrm{Li}$ 


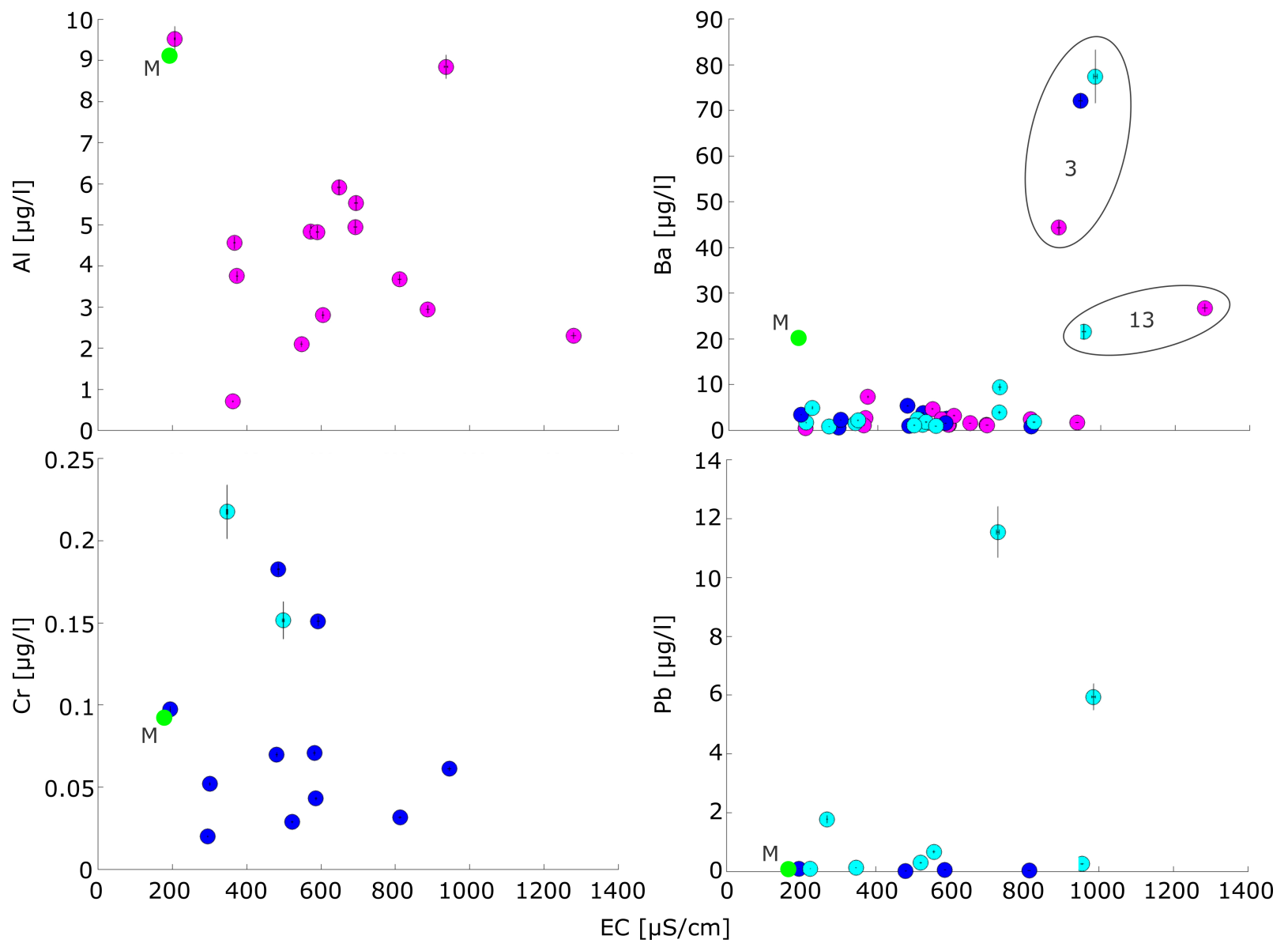

FIGURE 6.8: Trace metals for which data were available (reliable) are plotted against their associated EC measurements. Some of them could only be detected in summer (Al) or winter $(\mathrm{Cr}$ and $\mathrm{Pb})$. Their concentrations are generally low and seasonal variations in barium are marginal. For reference, the values of the trace elements are also plotted for the Mangone stream samples $(\mathrm{M})$.

and $\mathrm{Sr}$ found in all samples taken from bores 3 and 13 are another strong indication for the hypothesised remnant seawater lenses at these two locations. Furthermore, the clustering of bore samples for trace metals Li and Sr is very similar to the trends already observed for the major cations $\mathrm{Na}, \mathrm{Mg}$ and $\mathrm{Ca}$ in Fig. 6.6, indicating a positive relationship with these. From $\mathrm{Na} / \mathrm{Ca}$ ratios it had become clear that saltwater mixing can occur not only at bore locations with high TDS contents, but can also be detected in much less saline waters. Bores with relative stable $\mathrm{Na} / \mathrm{Ca}$ and $\mathrm{Mg} / \mathrm{Ca}$ ratios over time, as for example bore number 5 in Fig. 6.6, could not be entirely characterised. This bore shows one of the highest Sr concentrations measured all year round (Fig. 6.9), which leads to the conclusion that some seawater mixing must be present at this location at any time of the year. With a bore depth of $5.2 \mathrm{~m}$ below mean sea level it taps into the same zone as bores 2, 11 and 15. The missing seasonal component may be an indication for low throughflow at this location. 


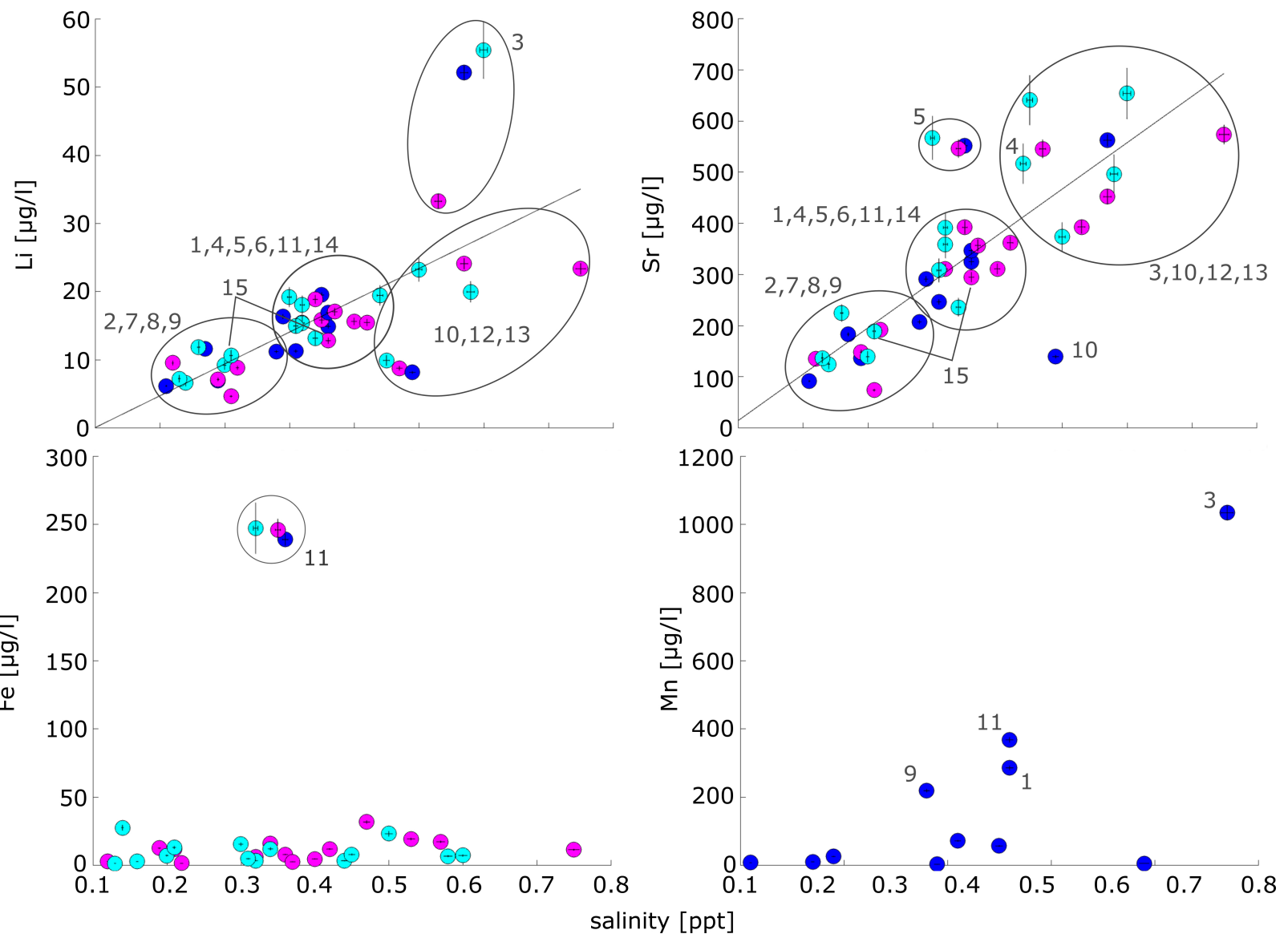

FIGURE 6.9: Seawater related (Li and $\mathrm{Sr}$ ) and non-seawater related (Fe and $\mathrm{Mn}$ ) trace metals plotted against salinity. Data quality is generally better for Li and $\mathrm{Sr}$ as a result of detectability. Positive relationships of the measured concentrations for trace metals in groundwater samples with salinity are apparent for lithium and strontium.

Iron and manganese concentrations are plotted against salinity in Fig. 6.9. Iron concentrations are below $50 \mu \mathrm{g} / 1$ for all bores and samplings except for bore number 11 which shows seasonally unchanged concentrations of around $250 \mu \mathrm{g} / \mathrm{l}$. Iron was also found in the Mangaone stream with an average concentration of $464 \mu \mathrm{g} / 1$. The close vicinity of the bore to the stream and the relatively high Fe concentrations in both samples could be an indication that the aquifer close to the stream is influenced by water discharge from the stream into the aquifer. Although Gyopari et al. (2014) states elevated Fe levels in aquifer unit Q4 in the Te Horo groundwater zone and there is assumed vertical groundwater seepage near the coast this is unlikely to be the source of the iron, because no elevated Fe concentrations were found in bore 18 even though the depth of this bore is $19.6 \mathrm{~m}$ below the mean sea level and therefore taps into a much deeper portion of the aquifer than bore $11(-2.7 \mathrm{~m})$. This also indicates that bore 18 (or the deeper Q1 aquifer parts in general) are most likely not influenced by surficial stream water exchange. Manganese was only found in winter 2015 samples. It usually coexists with iron in water but at 
lower concentrations (British Geological Survey, 2003). This is not possible to confirm for most bores as Fe concentrations were only obtained for 2016 measurements due to low detectability in winter 2015 samples. Bore 11, however, shows a clearly higher Mn concentration compared to its Fe concentration measured in June 2015. The amount of Mn in water is highly dependent on $\mathrm{pH}$ conditions and the abundance of oxygen in the water (aerobic/anaerobic conditions) and it is more stable towards oxidation than iron (Hem, 1985). This may explain the excess manganese concentrations in the groundwater of bore 11 compared to its iron content. High manganese concentrations are expected under anaerobic conditions in $\mathrm{pH}$ neutral systems where it is reduced to its soluble form $\mathrm{Mn}^{2+}$ (British Geological Survey, 2003). This could explain the high value measured in bore 3. No manganese was found in the Mangaone stream, and neither Fe, nor Mn show a trend with salinity. Relationships of Fe and Mn with $\mathrm{pH}$ and dissolved oxygen (DO) are further discussed in Appendix G. From these a seasonal dependence of $\mathrm{pH}$ was found throughout the aquifer with a lowered $\mathrm{pH}$ in the warmer season. A negative relationship between $\mathrm{pH}$ and $\mathrm{Mn}$ concentrations was found, which may also be tied to reducing conditions as indicated by DO measurements in the sampled bores. Generally, colder water can hold more DO, which was found to be the case in a few bores. However, most bores showed reasonably stable and low DO concentrations all year round. This points towards a reducing state of the shallow coastal aquifer and indicates a generally low flow of freshwater.

\subsubsection{Continuous electric conductivity, temperature and water level bore logging}

Between December 2015 and August 2016 it was possible to install a groundwater logger (CTDDiver with BaroDiver, Schlumberger Water Services (The Netherlands)) at location $R$ (Fig. 6.1) which continuously (every 15 minutes) measured groundwater EC, stage and temperature in the undisturbed (i.e. un-pumped) bore. The logger was placed at the bottom of the bore at a depth of $3.5 \mathrm{~m}$. Considering the elevation of the bore at the surface, this depth is equivalent to $0.3 \mathrm{~m}$ below mean sea level and the bore therefore taps into the middle layer of the shallow aquifer similar to bores 2, 11 and 15. In Fig. 6.10 the results for the entire monitoring period are shown. The generally observed small $\left(<2{ }^{\circ} \mathrm{C}\right)$ temperature variation of the groundwater between seasons is confirmed in bore $R$ as shown in Fig. 6.10(a) in orange. In the same figure, the groundwater EC is plotted, which, despite some fluctuations, shows a similar trend as 
the temperature with a general increase of EC from December 2015 to May 2016 and then a decrease again to August 2016. The highest air temperatures at the Kapiti Coast are generally expected in February (NIWA, 2014). However, the maximum groundwater temperatures are found between April and June. This indicates a time lag of approximately two months for groundwater temperature to adjust to air temperature. Because the 'true' EC was measured as opposed to the value corrected to $25^{\circ} \mathrm{C}$, these measurements may be influenced by temperature changes. A rule-of-thumb for the EC-temperature relationship is that EC varies by $2 \%$ for each $1{ }^{\circ} \mathrm{C}$ change in temperature (Van Essen Instruments, 2016). In Fig. 6.10(a) a temperature change of about $1.5^{\circ} \mathrm{C}$ is observed over the entire monitoring period. According to the above rule-of-thumb this would lead to EC changes of about $3 \%$, which for the measured conductivity would account to a value of less than $0.03 \mathrm{mS} / \mathrm{cm}$ (or $<0.6 \Omega \mathrm{m}$ for resistivity in Fig. 6.10(b)). This is considered negligible given that the accuracy of the logger is stated as $\pm 1 \%$ of the reading (Van Essen Instruments, 2016). It can therefore be assumed that the EC trend, which is similar to the temperature trend, is a real feature and not produced by temperature dependency of the EC measurements.

Short term fluctuations in EC observed during the entire monitoring period appear to be correlated to groundwater stage as illustrated in Fig. 6.10(b) and are independent of temperature. Groundwater stage varies between about 0.5 and $1.1 \mathrm{~m}$ above mean sea level between December 2015 and August 2016 and has generally lower levels during the summer months (Feb-May). The two sudden changes in groundwater stage in February and April 2016 are associated with two rain events in the dry season as is indicated in Fig. 6.3, whereas the steady increase from May onwards is attributed to a general increase in rainfall coming into winter 2016. Note that in Fig. 6.10(b) EC has been transformed to electrical resistivity in order to have better comparability with DC resistivity data. With a formation factor of 2.75 obtained for the study area (Wilson et al., 2006) the measured groundwater resistivity relates to a bulk resistivity of 30-55 $\Omega \mathrm{m}$ according to Eq. 3.28. This was considered to be sandy gravel saturated with a $1 \%$ seawater - freshwater mixture in Chapter 4 and matches interpretations from the hydrochemical analysis for the middle layer of the aquifer. Groundwater resistivity follows generally the same trend as groundwater stage, except for the time between February and June 2016 where they show a negative correlation (i.e. a decrease in resistivity follows after a short-term increase in groundwater level). This is contrary to the expectation that with more freshwater in the 


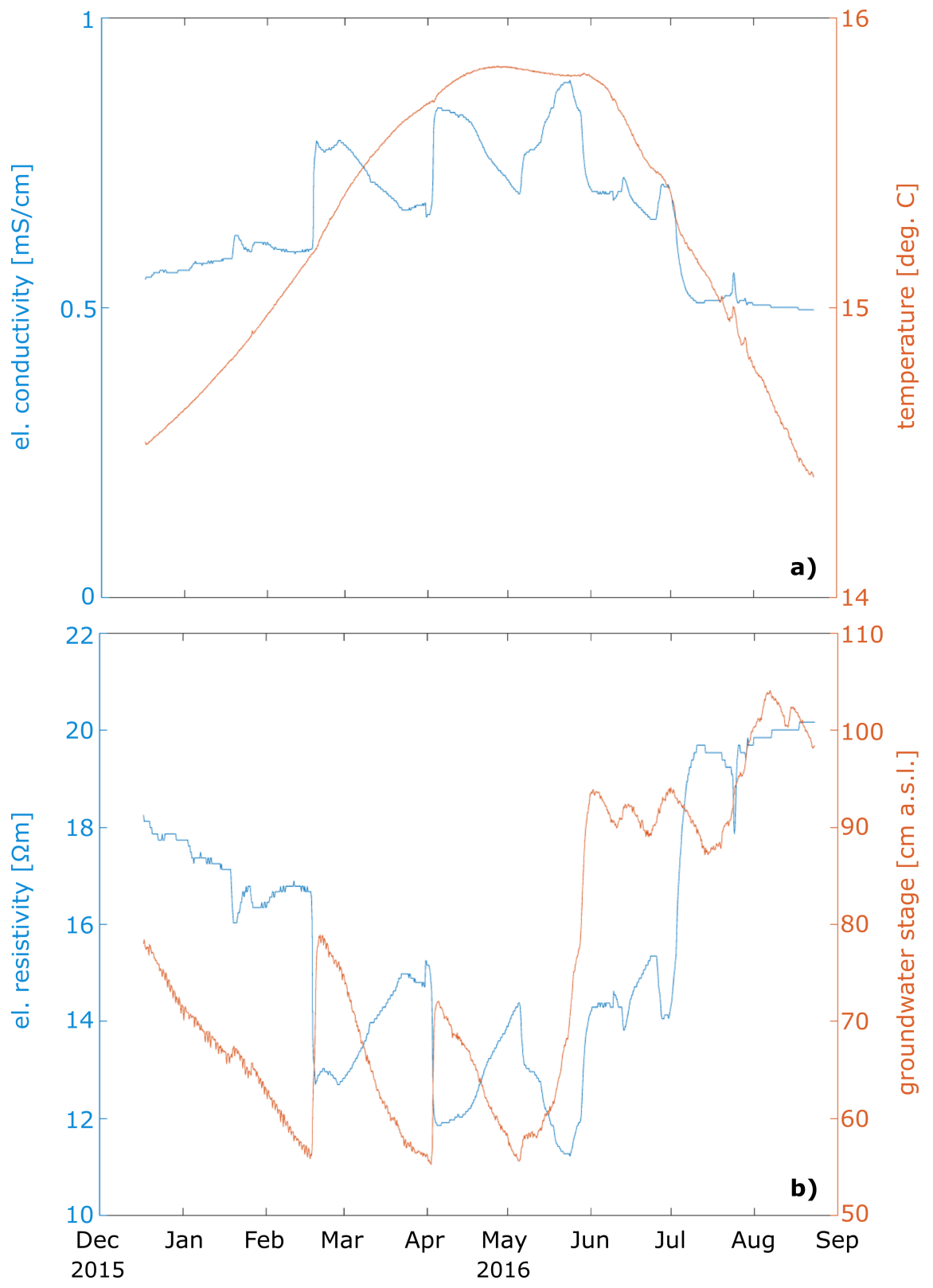

FIGURE 6.10: a) groundwater EC and temperature, and b) converted (=1/EC) groundwater resistivity and groundwater stage, continuously monitored in bore R (Fig. 6.1) between December 2015 and August 2016.

aquifer from rainfall recharge, the resistivity would increase due to dilution of the pore fluid. However, it would corroborate the theory that the dominant process in the shallow aquifer during summer is evapotranspiration leading to an accumulation of crystallised salts in the unsaturated pore matrix and a depletion of TDS in the groundwater. In such a system periodic rainfall events could dissolve these salt depositions and therefore temporarily increase the TDS concentration of the groundwater, which would lead to decreased resistivity (increased EC) readings. As the rain vanishes, evapotranspiration again becomes dominant, salts crystallise and the system goes back to its former state thereby increasing the resistivity of the groundwater. 
Once rainfall increases and evapotranspiration stops being the dominant process in this aquifer portion coming into winter, groundwater resistivity generally increases again with increasing freshwater amounts due to dissolution. This is in agreement with observations from the DC resistivity monitoring discussed in Section 4.5 and strengthens the conceptual model for feature 3 (gravelly layer) at this location.

\subsubsection{Estimating hydraulic conductivity from tidal amplitude attenuation}

The previously introduced bore monitoring data can also be used to estimate the hydraulic conductivity of the aquifer at the bore position by tidal amplitude attenuation. This method is also known as the Jacob-Ferris model (Jacob, 1950; Ferris, 1951) which provides a simple analytical solution to estimate hydraulic properties from groundwater stage fluctuations (Rotzoll et al., 2013). Initially, the model was developed for confined, homogeneous aquifers with a vertical boundary to the sea (i.e. a steep cliff), however, in the case where the aquifer thickness is much larger than the tidal range the model can also be applied to unconfined aquifers (Erskine, 1991). As this is the case in Te Horo, it was considered appropriate to use the Jacob-Ferris solution in order to get an estimate of hydraulic conductivity for the middle layer where bore $R$ taps into at the field site. The hydraulic conductivity $K$ can according to Rotzoll et al. (2013) be found from

$$
K=\frac{\pi S x^{2}}{b(\ln (A))^{2} \tau}
$$

with $S$ being the specific yield (in \%), $x$ the distance of the well to the coast, $b$ the saturated thickness of the aquifer, $A$ the ratio between groundwater and tidal stage amplitudes, and $\tau$ the oscillation period of the tidal signal. Specific yields of different geological material were measured by Johnson (1967). From analysis of the DC resistivity models in Chapter 4 the middle layer can be attributed to sandy gravel, for which Johnson (1967) suggests a specific yield of 0.25 . Bore $R$ has an approximated distance to the mean high tide mark of $207 \mathrm{~m}$ (Google Earth) and the saturated aquifer thickness can be estimated from the DC resistivity models in Fig. 4.7, bore logs in Fig. 2.8 and information from other studies (e.g. Gyopari et al., 2014) to be in the order of $25 \mathrm{~m}$ at the coast in Te Horo. From Fig. F.1 where the groundwater stage is plotted together with the tidal stage for two sequences of the entire monitoring period 
in bore $R$ it is apparent that the amplitude ratio approximates to 0.001 . The period of a tidal signal is in the order of 12 hours. Using these values in Eq. 6.1 gives an estimated hydraulic conductivity for the middle layer of $56 \mathrm{~m} /$ day. This is about two magnitudes larger than the $K$ estimated for the shallow coastal aquifer by Gyopari et al. (2014) and the difference may be attributed to the large heterogeneity of the aquifer close to the coast. It would, however, explain the lower resistivity values in the DC resistivity models (Chapter 4) and indicated seawater intrusion potential from groundwater sample analysis observed in this layer.

In Appendix B the hydraulic conductivity estimated for the gravel layer here, as well as the overall estimated small hydraulic conductivity of the shallow coastal aquifer through other authors, are used to calculate groundwater velocities of 2-50 cm/day within different geological materials. These are then compared to resistivity contour line movements obtained from the DC resistivity models discussed in Chapter 4 and it was found that the apparently hydraulically more conductive gravel layer with a relatively lower electrical resistivity has a higher horizontal water movement rate than any other part of the aquifer $(80-120 \mathrm{~cm} /$ day as compared to $1-5 \mathrm{~cm} /$ day for the bulk aquifer). This was found for both study sites and along all profiles and therefore indicates that hydrological conditions (i.e. the presence or absence of freshwater) of the aquifer are more important to resistivity changes than differences in the degree of urbanisation (though this, of course, potentially has a strong influence on how much freshwater is present in the aquifer).

\subsection{Geoelectrical monitoring results and their implications}

Time-lapse inversion results of the resistivity data for transect $\mathrm{TH} 2_{\text {ext }}$ are shown in Fig. 6.11. Also shown are plots of the resistivity ratios between subsequent time steps (February 2016/May 2015 and August 2016/February 2016) of the geoelectrical monitoring. The resistivity models show the same features as have been identified in Chapter 4, with the resistive surface layer (feature 1) representing semi-saturated to unsaturated sand with occasional gravel. Again, the thin surface layer of coarse gravel typical at the Te Horo Beach field site may influence local rainfall infiltration, but due to its small vertical extent, is most likely not resolved in the 5-m 
minimum spaced survey. The resistivity of the surface layer varies with groundwater level and moisture content related to short term precipitation or evapotranspiration conditions.

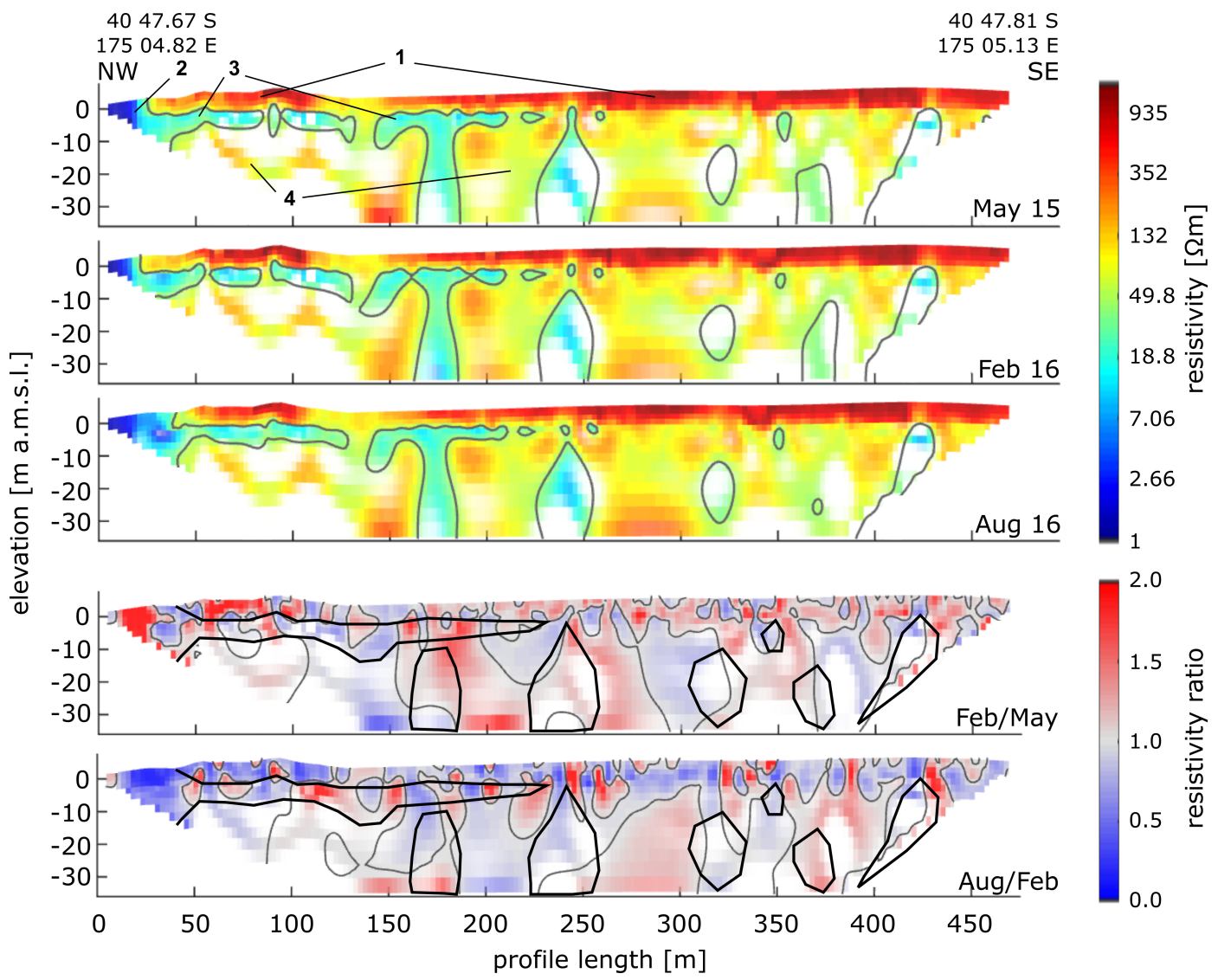

FIGURE 6.11: DC resistivity models and ratios made on three occasions (winter 2015, summer 2016 and winter 2016) on transect $\mathrm{TH} 2_{\text {ext }}$. Numbers 1 to 4 refer to the four features identified in the resistivity models in Chapter 4 and are discussed in the text. Black lines on the resistivity ratio images roughly outline the model parts showing low resistivity.

In the northwestern part of the profile the very low resistive zone (feature 2), marking the transition from fresh- to saline water, is captured. This again appears to be connected to a 5-10 $\mathrm{m}$ thick conductive layer (feature 3) likely representing the gravelly layer evident from the $\log$ obtained in bore 12 (R25/5267 in Fig. 2.8) adjacent to profile $\mathrm{TH} 2_{\text {ext }}$ at depths between about mean sea level and 5-10 m below mean sea level. The layer appears to become more discontinuous with increasing distance from the coast. Below this lower resistive zone, and further away from the coast directly underneath the resistive surface layer, a more resistive part of the aquifer is imaged in the resistivity models (feature 4). More compacted sands with occasional gravel lenses and saturated with freshwater is a possible explanation for the observed resistivity range at this depth interval and agrees well with the bore information found in $\mathrm{Te}$ Horo Beach as outlined in Section 2.4.2. 
The location of the boundary between saltwater saturated sands and more resistive features to the southeast appears to vary slightly with seasons. Contrary to expectations of seawater intrusion further inland during summer, the horizontal interface position shifts towards the coast from winter (May 2015) into summer (February 2016), and then away from the coast from summer to winter (August 2016). However, the second winter measurement in August 2016 was influenced by a significant storm in late July 2016 which produced wave run-up much further inland than normally observed. This led to seawater seepage downwards from the surface close to the coast and it is probable that this is largely responsible for the more extensive shift of the low resistivity region in the northwest, overprinting any seasonal pattern in the first $50 \mathrm{~m}$ of the profile.

From winter 2015 to summer 2016 the resistivity ratios (Feb/May) predominantly show an increase in resistivity above mean sea level. This includes a significant increase in resistivity in the upper $10 \mathrm{~m}$ in the area where the observed shift in the saline boundary occurs, as well as in the very near surface where reduced moisture content and a shallower groundwater level in summer raises the resistivity. In the thin conductive region below the surface layer up to about $200 \mathrm{~m}$ on the profile, changes are heterogeneous, but dominantly reflect an increase in resistivity, too. Deeper parts of the model space which also have lower resistivity and are up to a profile length of about $250 \mathrm{~m}$ also indicate a resistivity increase coming into summer. Those located more than $300 \mathrm{~m}$ from the coast generally show a decreased resistivity. However, depths down from about $10 \mathrm{~m}$ below mean sea level and further away from the coast have a relatively small resistivity ratio amplitude suggesting only little change in resistivity occurs between seasons. The patterns explained above for the transfer from winter into summer is fully reversed for going from summer into winter again (Aug/Feb).

Observations of seasonal resistivity changes made for the first 200-250 m along the extended TH2 profile in the Te Horo groundwater zone are in accordance with what was found on the shorter beach profiles discussed in Section 4.4.2 and their interpretation in Section 4.5 where freshwater forcing was found to be the major factor in resistivity shifts with season. It was also indicated that potentially enhanced evapotranspiration may have an influence on the freshwater mineral content in feature 3. Aquifer parts further than $300 \mathrm{~m}$ away from the 
shore appear to show the opposite temporal resistivity behaviour which can be interpreted purely with more or less freshwater being present in the aquifer as outlined in Fig. 1.2. However, the low amplitude in resistivity changes may point towards a system with small water flow and hence small changes in water chemistry (i.e. changes in electrical properties) over time.

The following section discusses the hydrochemical and resistivity measurements taken on three occasions during one year with respect of their combination. This can be used to constrain the resistivity models and at the same time extrapolate the in-situ hydrochemical measurements with greater certainty.

\subsection{Discussion and insights from combining time-lapse hy- drochemical and geoelectrical data}

The results from the hydrochemical monitoring study carried out in Te Horo Beach have been used to investigate several relationships in order to detect any indication of seawater mixing in the aquifer in general and within a seasonal context. Bore depth has been identified to be a major factor for dominance of different processes and saline intrusion potential. In Fig. 6.12 all bores sampled are imaged and coloured according to the depth category they tap into (green $=$ above mean sea level, orange $=$ between 0 and $5 \mathrm{~m}$ below mean sea level, pink $=$ deeper than $5 \mathrm{~m}$ below mean sea level). Close inspection of relationships between major cations measured at the field site leads to the conclusion that high TDS (EC, salinity) concentrations do not necessarily need to be an indicator for seasonally induced seawater mixing and that bores with lower TDS can be affected by saline intrusion. A better estimation of seawater intrusion potential may be the combined investigation of major cation ratios, especially the relationship between sodium and calcium, and saltwater indicating trace metals (i.e. strontium and lithium). Calcium plays an important role as its accumulation can be either due to higher weathering rates (i.e. from increased recharge), or as a consequence of seawater intruding and exchanging adsorbed calcium with sodium. Samples showing high sodium and calcium concentrations at the same time and also showing increased concentrations in these ions during summer time have a strong implication for seawater intrusion, whereas those having high calcium concentrations in winter are more likely affected by weathering. Furthermore, enriched 
trace metal concentrations such as for strontium and lithium are strong indicators of seawater because they are not abundant in large quantities in other water types.

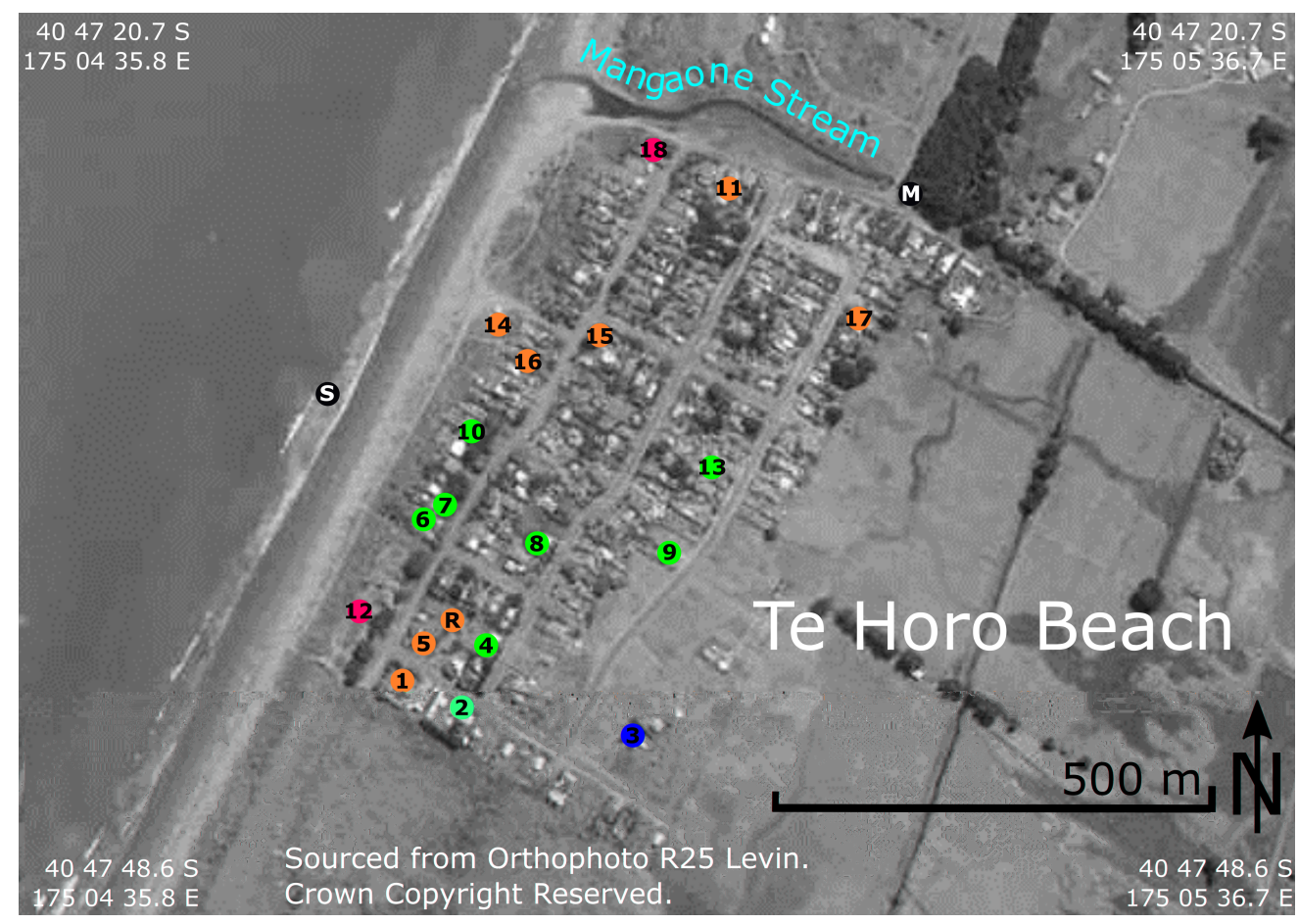

FIGURE 6.12: Sampled bores coloured according to their bore depth with respect to the mean sea level. Green $=$ above mean sea level, orange $=$ between 0 and $5 \mathrm{~m}$ below mean sea level, and pink $=$ deeper than $5 \mathrm{~m}$ below mean sea level. The two sampling locations $\mathrm{S}$ and $\mathrm{M}$ are surface waters and are hence not appointed a depth category.

From the discussion of the results of the hydrochemical study in Section 6.4, all bores have been assessed for their potential of seawater mixing, which is illustrated in Fig. 6.13. In order to qualify for the red category that indicates strong implications for seasonal saline mixing, samples needed to show seawater mixing potential from at least two independent parameters (e.g. high $\mathrm{Ca}$ and $\mathrm{Na}$ concentrations in summer plus a high content in strontium). Bores that are coloured green in Fig. 6.13 did not indicate saline intrusion. There was one bore (6) that was difficult to place into one of these two categories because it shows indications for both. The two blue bores were interpreted to tap into old stagnant saltwater lenses due to their high concentrations in TDS, Sr, Li and Ba. The three bores that were only measured once can only be interpreted to a certain extent and are therefore coloured in a lighter tone.

When Figs. 6.12 and 6.13 are compared, it quickly becomes clear that there must be a relationship between the saline intrusion potential and bore depth. From this it appears that bores 


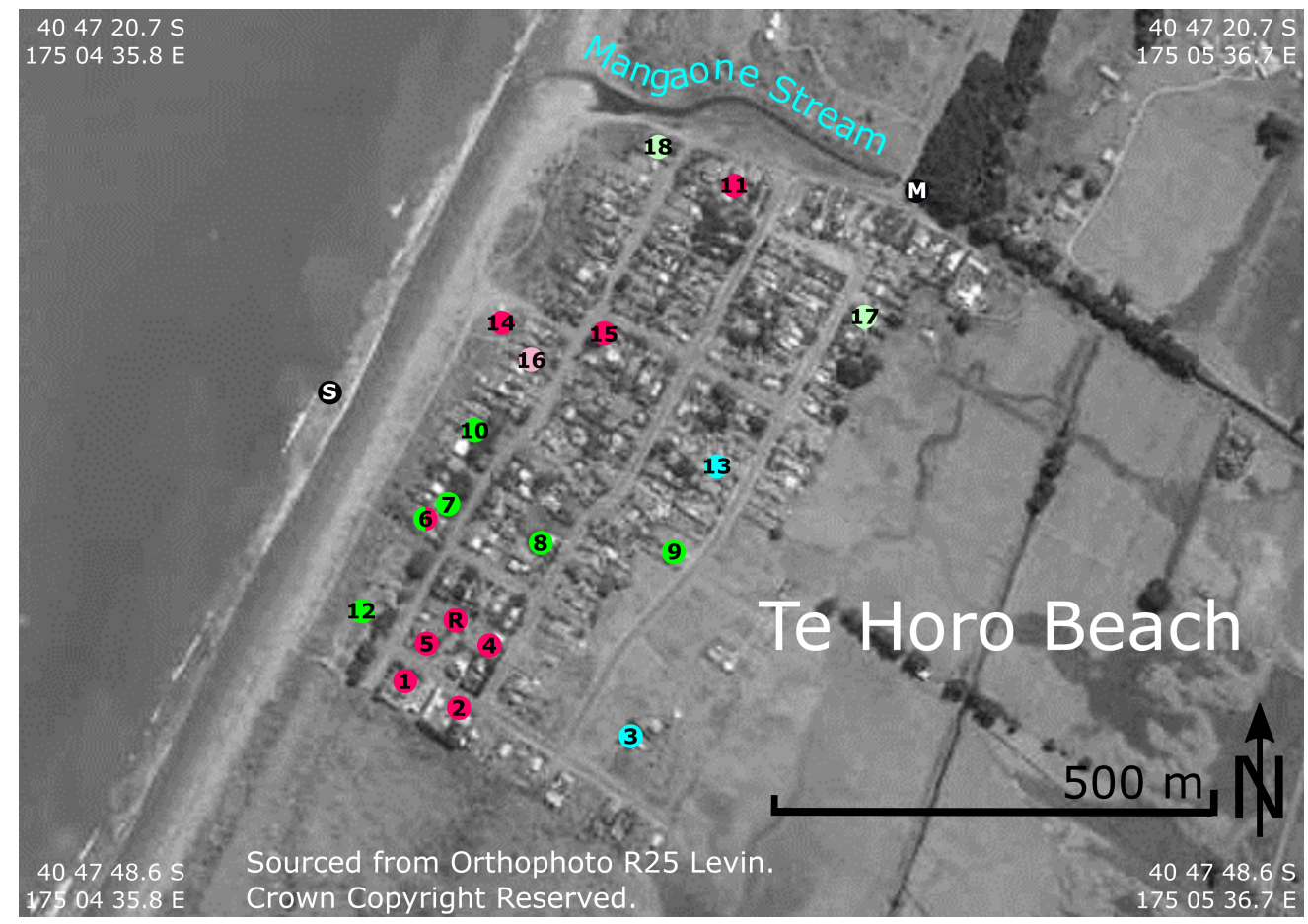

FIGURE 6.13: Sampled bores coloured according to their potential for seawater mixing. Pink dots show a tendency for seawater mixing in two or more of the measured parameters. Green dots indicate that there was little or no implication for seawater mixing. Blue dots mark bores that likely tap into old seawater lenses or stagnant old groundwater that is enriched in TDS. With pale coloured dots bores are marked for which only one sample was taken during the sampling period to indicate which behaviour in terms of seawater mixing it indicates based on that one sample.

with a depth between 0 and about $5 \mathrm{~m}$ below the mean sea level show strong indications for seawater mixing, while bores deeper or shallower than this do not indicate this. Only two bores (4 and 6) within $300 \mathrm{~m}$ of the shoreline do not distinctly follow this rule. However, their exact depths are unknown and were assumed to be $5 \mathrm{~m}$ (Table 6.3) meaning that they reach 0.1 and $0.5 \mathrm{~m}$ above mean sea level, respectively. It may well be that bore 4 actually belongs to the medium depth category rather than the shallow one. And the inconclusive results regarding seawater mixing potential for bore 6 could be due to the bore depth being within the transition zone between the shallow and medium depth category with changing aquifer conditions.

The above discussed categorisation of the aquifer into three depth levels according to hydrochemical indication of seawater intrusion potential in the tested bores agrees well with the geological bore information as displayed in Fig. 2.8. A near-surface semi-saturated to unsaturated sand layer overlaying an approximately $5 \mathrm{~m}$ thick gravelly horizon, with a more compacted sand body containing gravel lenses at depths larger than about 5-10 m below mean 
sea level. Though, heterogeneity of the aquifer stratigraphy needs to be kept in mind, the indicated hydrogeologic situation is also reflected in the DC resistivity profiles collected in the Te Horo groundwater zone. This strongly suggests that seawater is entering the shallow coastal aquifer predominantly through the potentially hydraulically more conductive gravel layer. This conclusion was also drawn from the results of the tidal monitoring investigations as discussed in Chapter 5. The relationship between groundwater electrical conductivity (transformed to bulk resistivity with the formation factor of 2.75 (Wilson et al., 2006)) and DC resistivity models for the three sampling dates is discussed in the following and further investigates the robustness of their comparison.

Although the superimposed EC data from the wells appear to match well with the DC resistivity models in Fig. 6.14, the horizontal errors of the well positions are large at some locations due to projection and location inaccuracies, and therefore lead to some ambiguity. This stresses the importance of having good knowledge of well depths and locations for optimal comparison of the two methods. However, absence of drilling information and bore pumps blocking the bores for direct in-well measurements made this quite difficult for some of the sampling locations in this study. Also the absence of bores reaching larger depths, only allows comparison of the uppermost approximately $10 \mathrm{~m}$ of the aquifer. However, this nicely illustrates the findings from the hydrochemical study, where a correlation between bore depth and seawater intrusion potential was drawn for bores located between 0 and $5 \mathrm{~m}$ below mean sea level. From Fig. 6.14 it becomes clear that this affects the bores tapping into the low conductivity zone at approximately this depth interval, but that this zone only extends to about 200-250 m inland. From the hydrochemical analyses it was also concluded that seawater intrusion is only apparent to about $300 \mathrm{~m}$ from the shoreline. An observation also made on the resistivity ratios along profile $\mathrm{TH} 2_{\text {ext }}$ in Fig. 6.11.

From groundwater stage fluctuations measured in bore $R$ a hydraulic conductivity of $56 \mathrm{~m} /$ day was estimated for this layer using the approach of tidal amplitude attenuation by Rotzoll et al. (2013) in Section 6.4.3. This is relatively high compared to the estimate of $2-5 \mathrm{~m} /$ day of the shallow coastal aquifer found from other studies in the area (e.g. Gyopari et al., 2014), but would explain a hydraulically better conducting layer at this depth interval, such as gravel and coarse sand as is indicated for example by the driller's log of bore 12 (see R25/5267 in 


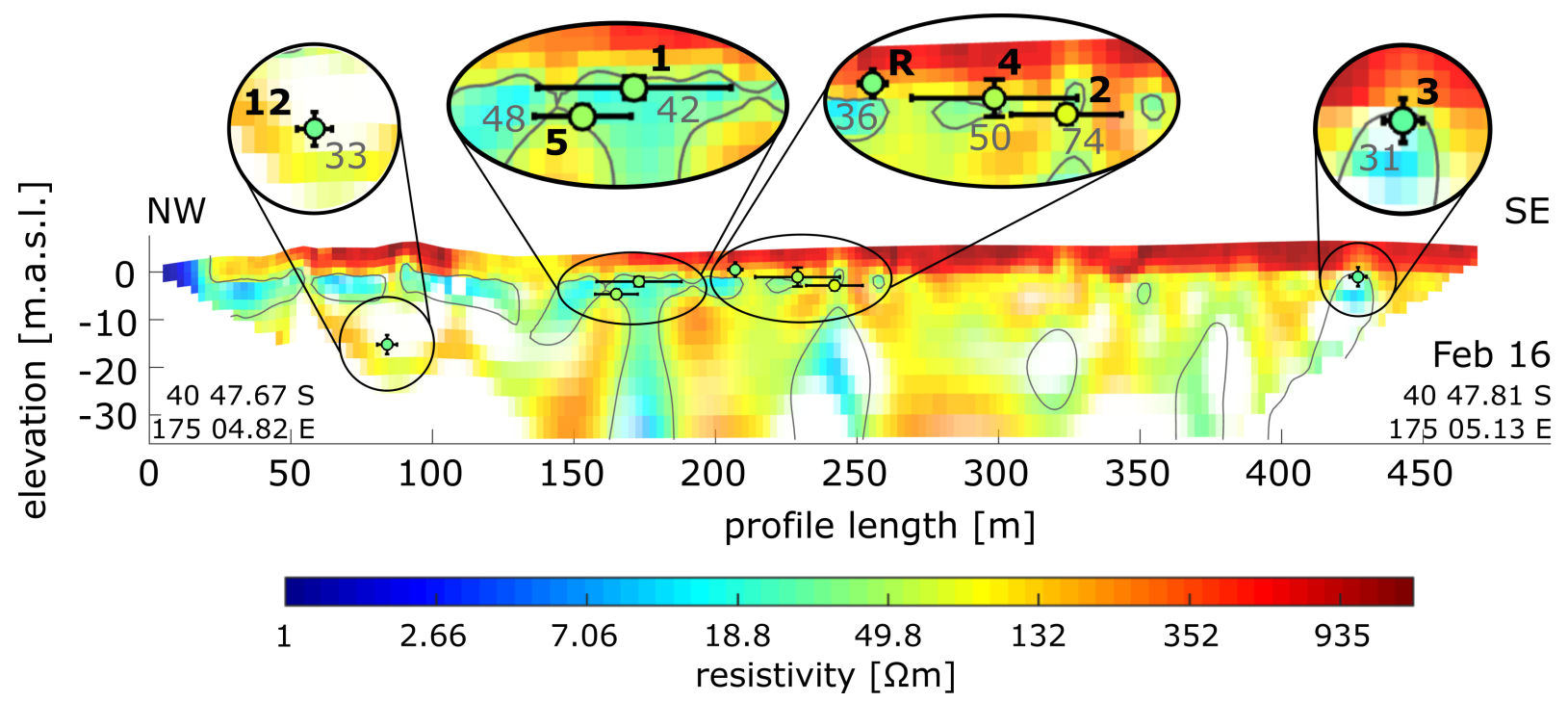

FIGURE 6.14: Resistivity model (along extended TH2 profile in Fig. 6.1) and to bulk resistivity transformed bore water EC measurements (dots with error bars) of the February 2016 groundwater sampling campaign. Both use the same colour scale. Bore numbers as assigned in Chapter 6 are given with bold black numbers and the bore water bulk resistivity values are stated as grey numbers (in $\Omega \mathrm{m}$ ). The absolute values of the two methods agree well when superimposed and can be used to constrain each other.

Fig. 2.8). A higher hydraulic conductivity could significantly lower the measured resistivity due to increased infiltration of seawater within this layer up to about $250 \mathrm{~m}$ profile length as indicated in Fig. 6.14. The extended TH2 profile also illustrates the high heterogeneity of the shallow aquifer and furthermore strengthens the theory that bores showing low EC values and high total dissolved solids (TDS) contents more than $300 \mathrm{~m}$ inland (i.e. bores 3 and 13) are remnants of old seawater inclusions from a prograding coast line, as in Fig. 6.14 the location of bore number 3 appears not to be in direct connection with the low-resistivity layer closer to the coast due to discontinuity of this layer with increasing distance from the coast.

Because transect $\mathrm{TH}_{2}$ ext was measured three times during the course of a year, it is possible to image the resistivity ratios together with the EC ratios between winter and summer months (i.e. summer 16/winter 15 and winter 16/summer 16) as illustrated in Fig. 6.15. In terms of seasonal changes the two methods agree relatively well and show the same increase or decrease behaviour within errors. For example, in the Feb/May-ratio plot in Fig. 6.15 the groundwater EC ratio of bores 1 and 2 show a decrease in electric conductivity of $10 \%$ and $50 \%$, respectively, but they are coming to lie within areas of a resistivity increase in the DC resistivity models. However, due to projection and bore location errors, the actual bore values could also be located to the left or right of the indicated posisiton, where they would match very well with 
the resistivity ratio obtained from the geoelectrical models. Therefore, while the hydrochemical study of the bore water samples was able to determine seawater intrusion at the field site as a function of bore depth, this estimate was rather rough due to uncertainties resulting from the fact that some of the bore depths were not known accurately. By combining it with the geoelectrical data, correlation of the two methods in absolute and ratio value perspectives, it is possible to overcome these positioning errors. This results in a much more accurate estimate of a preferential seawater intrusion pathway within the shallow coastal aquifer.

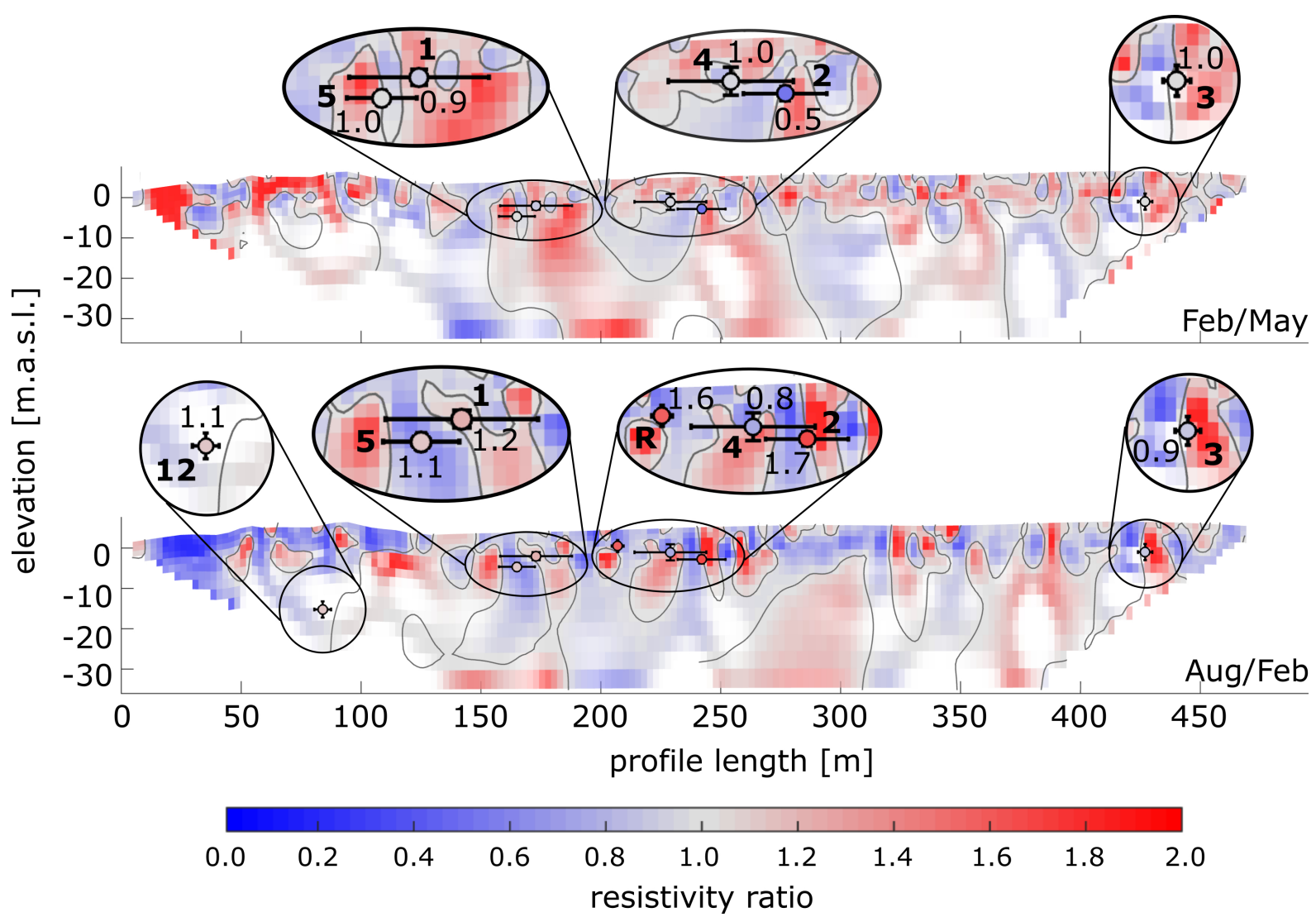

FIGURE 6.15: Resistivity and EC ratios superimposed on each other for the extended profile TH2 in Te Horo (Fig. 6.1). Bore numbers are indicated in bold, whereas the groundwater EC ratios are stated with light numbers. Note that the colour scale is the same for the hydrological and geophysical data. The results of the two methods show good agreement within errors.

Measurements of DC resistivity and groundwater parameters were also measured combined by Wilson (2003) at similar locations in Te Horo Beach. Although these measurements were not done repetitively at the time, they can now serve as a record to investigate seawater intrusion potential into the shallow coastal aquifer over a longer time span. This is discussed in the following section. 


\subsection{Long-term implications for seawater intrusion in Te Horo Beach}

Te Horo has experienced a major growth in the past ten years with an increase in population of $20 \%$ between the census years 2006 and 2013 alone (www.stats.govt.nz). And although these numbers include the entirety of Te Horo, it is a good indication for the growth rate in the Te Horo Beach settlement during these year. This is easily visible when comparing the 2005 and 2016 satellite images of Te Horo Beach in Google Earth. In Fig. 6.16 this has been done by marking all houses on the 2005 satellite image with black squares, whereas those additionally visible in the 2016 satellite image have been marked with a green square. Within eleven years the township has therefore grown by $24 \%$ in properties based on these images. According to the GWRC well and bore consent data base (http://mapping.gw.govt.nz) there were 54 wells and bores installed in the Te Horo Beach settlement until the end of 2005. This number increased by at least $20 \%$ in the eleven years after that. The bore numbers in this data base are most likely lower than the actual number of bores drilled in the settlement because those reaching depths shallower than seven meters do not need a consent and are therefore unlikely to show up in the GWRC data record. A clear indication of this is that several of the actually sampled bores during the 2016 groundwater sampling study are not registered in the data base but are obviously existent and in use. Therefore, a substantial increase in use of groundwater in Te Horo Beach within the past ten to twelve years is strongly indicated.

Concerns of residents about groundwater quality in the settlement led to an extensive bore water analysis initiated by the regional council in 1998 (Hughes, 1998). A Master's thesis at Victoria University of Wellington further investigated seawater intrusion at the Te Horo Beach field site in the years 2002 and 2003 (Wilson, 2003). This provides in situ EC and local geoelectrical information at this location for the past 13-18 years. In the following the available data is therefore compared within this time span in order to find possible links between groundwater extraction and seawater intrusion. The groundwater level in a deep bore (R25/0003) on Sims Road has been measured since 1985 and therefore a comparable groundwater level for all three measurement periods could be obtained. These are listed in Table 6.6. It shows typical groundwater levels in the field area with January 1998 being highest, while February 2016 


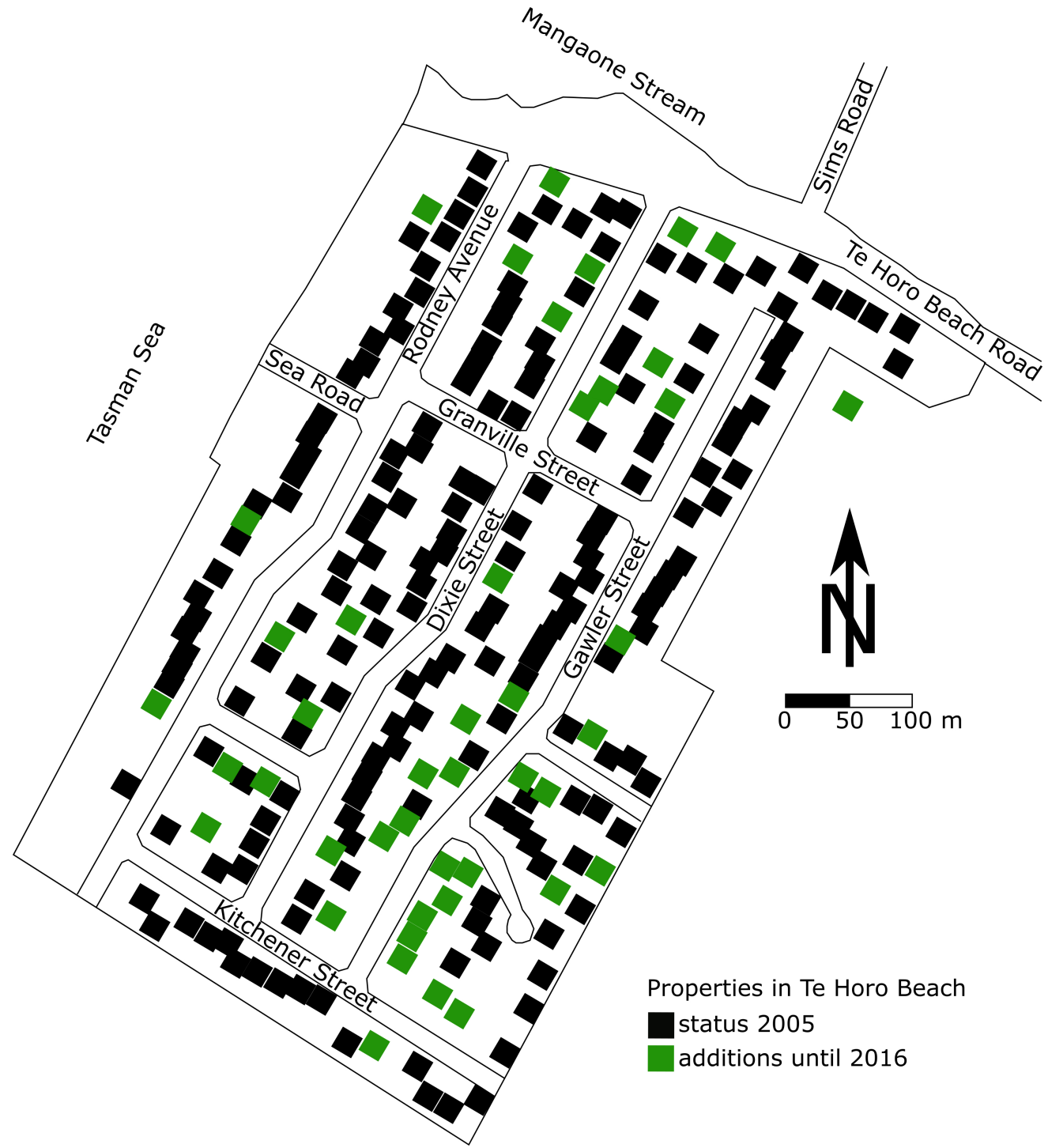

FIGURE 6.16: Te Horo Beach properties existing as indicated from the Google Earth 2005 satellite image (black) versus the one of the 2016 update (green). Each square marks one house.

shows a lower value and the lowest stage is found to be in April 2003. However, the minimum and maximum values do not differ by more than $200 \mathrm{~mm}$ and therefore it can be assumed that the three studies were acquired in approximately similar hydraulic conditions.

From the bore sampling study in 1998, Hughes (1998) observed a large spatial variability in the aquifer from measurements of several species (i.e. between bore samples only $200 \mathrm{~m}$ apart), and found an increasing redox potential of the aquifer towards the coast, which was tied to low groundwater flow in the area. However, bore depths are not stated explicitly ('predominantly shallow'), which may lead to a perception bias of the Hughes (1998) results, because, as seen in Chapter 6, the bore depth was found to be crucial for aquifer behaviour close to the coast at this location. In Fig. 6.17 the measurements of groundwater EC in several bores in Te Horo 
TABLE 6.6: Groundwater stage in well R25/0003 on Sims Road in Te Horo Beach (GWRC monitoring network) for the three electrical conductivity surveys. Note that these are average values for the time spans given in 'Sampling Date'.

\begin{tabular}{l|l}
\hline Sampling date & Groundwater stage $[\mathrm{mm}]$ \\
\hline 6. - 8. January 1998 & 2218 \\
April 2003 & 2065 \\
23. - 24. February 2016 & 2107 \\
\hline
\end{tabular}

Beach of all three surveys are plotted. They are grouped into ranges of $200 \mu \mathrm{S} / \mathrm{cm}$ and, except for two bores along Rodney Avenue which show an increase in EC of 200-400 $\mu \mathrm{S} / \mathrm{cm}$, mostly have not changed within the 18 years between the first and the last measurements in the same bores. However, considering the high heterogeneity of the shallow aquifer and the unknown exact depths for many of the bores it becomes difficult to compare different bore locations with each other. In general, Fig. 6.17 illustrates an increasing EC trend towards the coast along the east-west axis for all three survey dates. Furthermore, the two bores (3 and 13) in this study that are located in the east of the settlement and show rather high EC values can now be put into context with surrounding bores for which generally very low EC measurements were obtained. This strengthens the stagnant saline water inclusion hypothesis at these locations. Bores closest to the Mangaone stream have higher EC values than those further away at the same distance away from the coast. According to Robertson and Stevens (2007) there is periodic saline influence depending on the extent of tidal inflow and amount of mouth blocking from driftwood of the Mangaone stream estuarine system up to around where Sims Road bridge crosses (approximately same extent as position of well R25/0003). Intrusion of more saline water into the aquifer from the stream could therefore be an explanation for this observation. It may also indicate higher hydraulic conductivity of river gravel deposits as was suggested by Gyopari et al. (2014) for the Waikanae groundwater zone (i.e. close to the Waikanae River), which could serve as a preferential path for seawater to intrude further inland.

Wilson (2003) additionally obtained EC monitoring data in three bores along Sims Road and the one about $150 \mathrm{~m}$ away from the shoreline (as opposed to the two others at $>300 \mathrm{~m}$ distance from the coast) showed seasonal variations similar to the ones observed during the seasonal groundwater sampling of the current study (i.e. higher EC in winter than summer). In his conclusion Wilson (2003) suggested this may be due to a six-month time lag in the aquifer. With a depth of 5.6-16 m below surface this bore most likely taps into the layer identified as the coarse gravelly sand in the middle of the coast-proximal part of the shallow aquifer. From 
the hydrochemical data analysis it becomes clear that apart from EC values, the data shows a seasonal component indicating increased seawater intrusion within this layer during the drier summer months. A time lag of half a year seems therefore unrealistic.

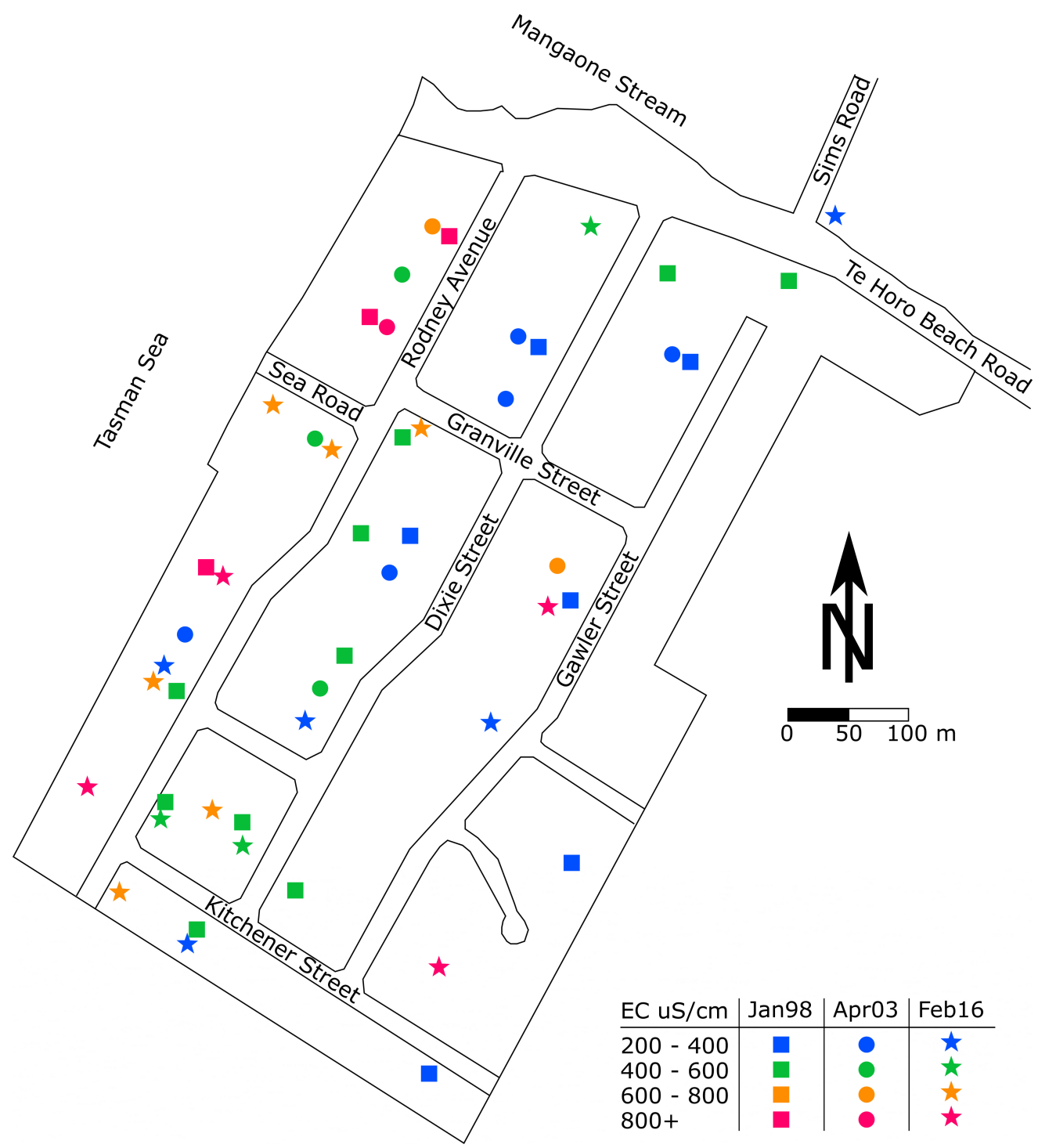

FIGURE 6.17: Comparison of three studies that measured electrical conductivity in bores around the Te Horo Beach settlement. Data for January 1998 are from Hughes (1998), those for April 2003 were taken from Wilson (2003), and those of February 2016 are from the current groundwater study (see also Chapter 6).

Following Hughes (1998) the EC values of all three studies have been plotted as a function of distance to the mean high tide mark in Fig. 6.18. Note that the original data for the April 2003 study (Wilson, 2003) could not be obtained and therefore the averages of the EC ranges in Fig. 6.17 were taken for this data set. Hence, the error on these may be up to $\pm 100 \mu \mathrm{S} / \mathrm{cm}$. The relationship of groundwater electrical conductivity relative to its distance from the coast 
for all three survey years in the past 18 years shows a light exponential trend with increasing EC coming closer to the shoreline (dashed line in Fig. 6.18). This is regardless of bore depth and makes sense as it would be expected that aquifer portions closer to the coast naturally experience a higher concentration in total dissolved solids due to their proximity to the sea. A more concerning observation is the one of a clear increase of bore samples found in the EC range $>600 \mu \mathrm{S} / \mathrm{cm}$ to a further distance away from the coast for the February 2016 survey (note the two high-EC values at distances $>350 \mathrm{~m}$ mark bores 3 and 13 and are most likely tied to old saline water inclusions rather than current saline intrusion) as compared to the other two field campaigns. This could be interpreted as saline water encroaching further inland between 2003 and 2016 and may be a direct consequence of the $20-24 \%$ increase in population and bores at the field site in Te Horo Beach.

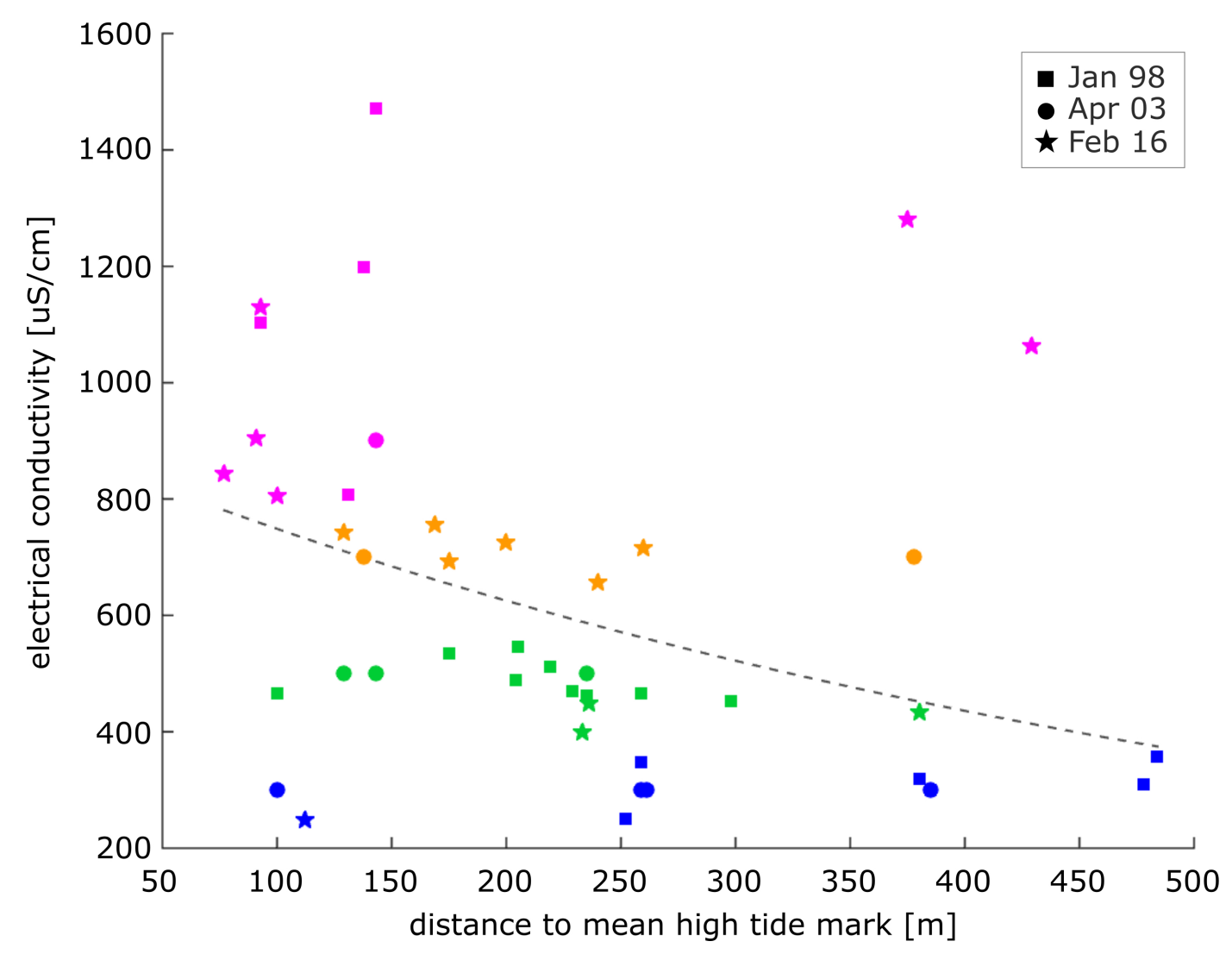

FIGURE 6.18: Comparison of the three studies in Te Horo Beach measuring EC vs. distance from coast. Colours refer to EC ranges used in Fig. 6.17 (blue $=200-400 \mu \mathrm{S} / \mathrm{cm}$, green $=400-600$ $\mu \mathrm{S} / \mathrm{cm}$, orange $=600-800 \mu \mathrm{S} / \mathrm{cm}$, pink $=>800 \mu \mathrm{S} / \mathrm{cm})$. Note that errors on April 2003 data may be up to $100 \mu \mathrm{S} / \mathrm{cm}$, whereas those from January 1998 and February 2016 are in the order of $1 \mu \mathrm{S} / \mathrm{cm}$.

Wilson (2003) also used the geoelectrical method in his thesis and, amongst other locations, also measured the TH1 transect in Te Horo in January 2003. The data was inverted with the 
same program, inversion procedures and parameters as used during the current project in order to be comparable to the January 2016 data set obtained from the TH1 transect. In similar hydrological conditions (i.e. similar groundwater stages) the saline interface in 2016 seems to be shifted approximately $4.5 \mathrm{~m}$ further inland from its position in 2003 at the surface, and shows a shift of about $0.5 \mathrm{~m}$ at a depth of approximately ten meters below mean sea level (Fig. 6.19). Additionally, the parts of the aquifer where more saline water is detected (i.e. gravelly sand middle layer between about 0 to $10 \mathrm{~m}$ below mean sea level with resistivity values of less than $50 \Omega \mathrm{m}$ ) appear to be more continuous in the 2016 resistivity model, which may be a consequence of more saline water infiltrating this layer. However, the data quality of the 2003 survey appears to be lower than that for the 2016 data set as indicated by the number of blanked out values after using the DOI model appraisal analysis, and this therefore may introduce some errors in determining the resistivity isolines and lead to a biased impression. The resistivity ratio between the two resistivity models (for comparable parts) in Fig. 6.19 almost exclusively shows a decrease in resistivity of about 40-60 \% between January 2003 and January 2016 throughout the entire model space. Considering that the groundwater stage during the 2016 acquisition actually was about $70 \mathrm{~mm}$ higher than during the 2003 survey, this further points towards increased salinization of the shallow coastal aquifer in Te Horo within the 13 years between the two geoelectrical measurements.

\subsection{Summary}

Ion concentration variations measured in 18 bores suggest seawater intrusion up to about 200 $\mathrm{m}$ from the coast and at depths between $0 \mathrm{~m}$ and 5-10 $\mathrm{m}$ below mean sea level during summer. Elevated concentrations of iron were found in the Mangaone stream and one bore that taps into above depth interval and is adjacent to the stream. This is an indication of river water - freshwater exchange close to the steam where their geologic units are connected. Oxygen depleted groundwater was sampled in two bores which also show unusually high total dissolved solids concentrations. These were located a further distance away from the coast and were interpreted as old saline lenses from a past prograding coastline. A conclusion also drawn from DC resistivity monitoring in Chapter 4. From continuous groundwater stage and EC logging in a bore reaching into the geological horizon at 0-10 $\mathrm{m}$ depth below mean sea level, the from the 


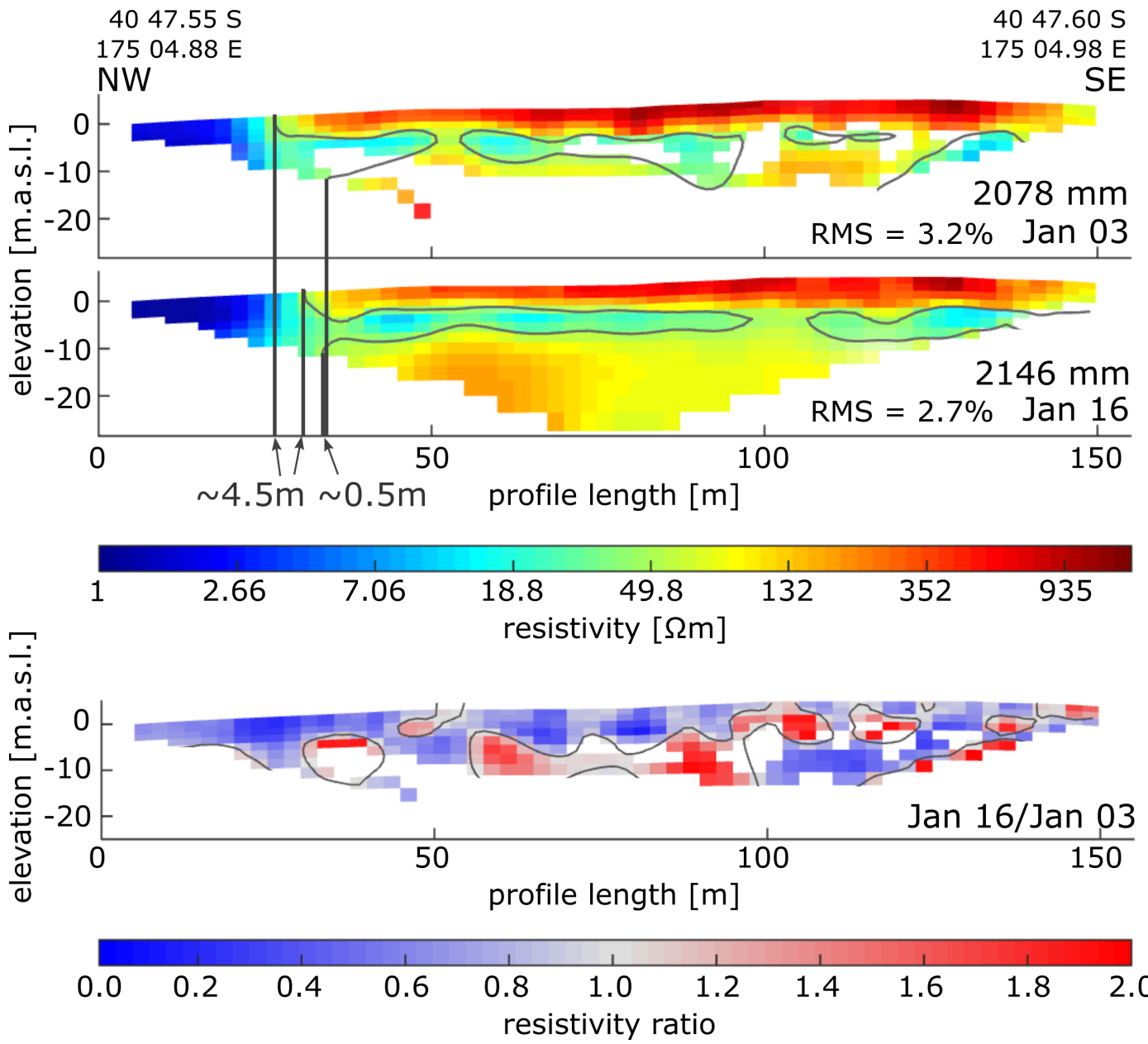

FIGURE 6.19: TH1 profile measured in January 2003 for the study carried out by Wilson (2003) and the same profile measured in January 2016 from the Te Horo Beach seasonal monitoring data set (13 years later). The contour lines indicate the $45 \Omega \mathrm{m}$ isoline which is considered about $1 \%$ saltwater mixing (as introduced in Chapter 4). At the bottom the resistivity ratio between the two inversion models is shown for model cells that exist in both data sets.

main hypothesis unexpected relationship during summer months of these two parameters can be explained by the same processes already indicated in Section 4.5 and therefore strengthens the hypothesis of a freshwater pressure dominated system with influence of enhanced evapotranspiration rates during summer. A higher hydraulic conductivity estimated from tidal amplitude attenuation calculations for the thin layer just below mean sea level, is another indication of seawater preferentially moving along this feature in the horizontal direction.

Using the propagation of resistivity isolines from geoelectrical monitoring models, a velocity of saltwater mixing at the field locations was estimated in Appendix B to be in the order of $1-5 \mathrm{~cm} /$ day from seasonal observations and $80-150 \mathrm{~cm} /$ day from tidal monitoring models. The discrepancy between the different velocities is possibly a consequence of the tidal monitoring 
resolving better a much larger portion of a hydraulically much more conductive layer (i.e. feature 3) with an estimated hydraulic conductivity of $56 \mathrm{~m} /$ day, as compared to the seasonal profiles which contain a larger portion of the hydraulically less conductive fine to medium grained sand ( $K \sim 2-5 \mathrm{~m}$ /day (Gyopari et al., 2014)). From Darcy's law groundwater velocities of $22 \mathrm{~cm} /$ day and $1.4 \mathrm{~cm} /$ day for the hydraulically more or less conductive geologic materials have been calculated, respectively. These agree reasonably well with the obtained saline mixing velocity estimates found from the seasonal and tidal observations. In general, water movement velocities are found to be smaller for vertical flow than for flow in the horizontal direction, which suggests a dominant groundwater flow and seawater mixing horizontally, rather than vertically. A conclusion drawn also by Gyopari et al. (2014) that found a vertical hydraulic conductivity two magnitudes smaller compared to the horizontal one for this aquifer.

The extended geoelectrical profile $\mathrm{TH} 2_{\text {ext }}$ indicated the lower resistive zone termed feature 3 to become more discontinuous with distances more than $250 \mathrm{~m}$ away from the shore. Parts of the resistivity models that show lower resistivity values appear disconnected from the saline zone (feature 2) at the northwestern end of the profile and were interpreted as being remnants of old saline water. This again agrees with high ion concentrations found for bores located within this part of the aquifer as discussed above. Resistivity ratios between winter and summer measurements show different temporal behaviour of the aquifer along the first $250 \mathrm{~m}$ and the second portion of the profile extension in comparable resistivity zones (e.g. gravel layer / lenses). However, parts further away from the coast and at larger depths showed lower amplitudes in resistivity change. These are indications that the aquifer is dominated by freshwater. Parts that have an absence of the hydraulically conductive gravel layer connected to the sea show the expected decrease in resistivity coming into summer when there is less freshwater present and vice versa when coming into the winter season with enhanced rainfall. The hypothesis first introduced in Chapter 4 is once more able to explain why the coast proximal part of the model reacts in the opposite way. The low amplitude of the resistivity changes in some parts along the profile are indicative for a generally low hydraulic conductivity of the bulk aquifer.

Superposition of bore groundwater electrical conductivity measurements onto DC resistivity profiles extending profile TH2 in Te Horo to $495 \mathrm{~m}$ in total, showed graphically good agreement between the hydrological and geophysical measurements within projection and bore 
depth errors. This further strengthened the individual findings of the two methods of indicated larger seawater intrusion into the coarse gravelly layer (also termed feature 3) between approximately mean sea level and to 5 to $10 \mathrm{~m}$ below mean sea level. While the in situ hydrological measurements provide a ground-truth value, the geophysical models are able to extend this information on a broader scale. This way a better constrained model of resistivity distribution and hydrological meaning of the resistivity values is obtained.

Finally, a comparison between hydrological and geoelectrical studies carried out over the past 18 years in Te Horo Beach strongly implied that there is increased seawater intrusion at the field location within the past decade or so. This is observed to be parallel to a 20-24 $\%$ increase in population (property and consented wells) and hence, strongly suggests that seawater intrusion into the shallow coastal aquifer within this time is tied to the increase in groundwater use from shallow bores (i.e. related to a freshwater pressure decrease). A mean sea level rise of about $1.3 \mathrm{~mm} /$ year (Bell et al., 2000) may be an additional factor (rise of $17 \mathrm{~mm}$ between 2003 and 2016), however, considering the small changes caused within a tidal cycle on the groundwater EC measured in bore $R$ (see Section 6.4.2) which have groundwater head differences several magnitudes larger than this, it is more likely that the observed increased seawater intrusion is mainly caused by higher freshwater abstraction.

All of the above measurements and calculations indicate the same scenario: A hydraulically more conductive layer that coincides with a gravelly sand interval, as indicated from bore core information, at a depth of about mean sea level to 5-10 m below mean sea level. This layer appears to have higher horizontal groundwater movement associated and acts as a preferential flow path of seawater further inland in the case of a lowered freshwater pressure. In sustainable groundwater management it is very important to know whether such features exist close to potential or existing abstraction points. Combining classical hydrological sampling strategies with geoelectrical monitoring in a repetitive manner clearly leads to a better constrained interpretation of such features. 



\section{CHAPTER 7}

\section{Integration of information: A conceptual model of a shallow coastal aquifer}

"No one ever made a decision because of a number. They need a story."

Daniel Kahneman, psychologist and Nobel Prize winner (1934 - present)

In the final chapter of this thesis I integrate available and newly obtained information from geoelectrical, hydrochemical and modelling results into a conceptual model of the shallow coastal aquifer at the Kapiti Coast. I will summarise the findings and implications and outline how these integrate with the dynamics of the groundwater system, thereby providing conclusions that can be drawn from this project. I end with an outlook for suggested further work. 


\subsection{Introduction}

Groundwater with its many advantages over surface water as a freshwater supply (e.g. large amounts often protected from direct contamination), is nevertheless subject to contamination threats and overexploitation. One of the future (and present) concerns with coastal groundwater sources is contamination of the source with seawater from either rising sea levels or excessive groundwater pumping from wells located close to the coast. In order to avoid seawater intrusion and develop a sustainable use for freshwater supply in these areas it is necessary to understand the short and long term dynamics that influence groundwater recharge and discharge in such environments. This thesis was looking into the near-coastal processes between fresh- and seawater exchange underground within two time frames - tidal and seasonal - and using the advantage of geophysics to gain a more extended view of the subsurface, while having in situ measurements from hydrochemical water analyses to get a different angle of view for a better overall understanding. In the previous chapters the outcomes of these studies were discussed thoroughly and provide vital additional information concerning the dynamics of the seawater-freshwater system in the study area. However, it is hard to keep an overview of all the information available and ultimately gain a deeper understanding if it is not combined in a sensible way.

The problem can be simplified by constructing a conceptual model that describes the physical components and interaction of the groundwater system. This way the available information can be organised and integrated for a wider understanding of processes that are interrelated and hence, accurately analyse the system. A good hydrological conceptual model that can later be used for numerical modelling needs to discuss three key components according to Fulton et al. (2005):

1. Surface water - groundwater interaction,

2. physical exchange of water, and

3. processes that influence recharge and discharge/abstraction in the area.

With regard to variable density flow situations such as seawater-freshwater mixing in a coastal aquifer it is of additional importance to consider density driven flow and circulation which 
contribute to the fluid dynamics in the mixing zone.

In a later step such a conceptual model can be used in addition to the data in order to develop a more detailed numerical model (using programs such as the MODFLOW/MT3DMS based SEAWAT code (U.S. Geological Survey) for simulation of three-dimensional variable-density groundwater flow and transport) in order to simulate seawater intrusion into the coastal aquifer, which links all components that are driving forces to the problem. Policy and decision makers would then have an accurate tool for sustainable groundwater resource management in geologically similar coastal areas.

This is the concluding chapter of the PhD thesis. Conclusions drawn from the three-year project will be illustrated and discussed within the framework of the conceptual model presented in the following section. All implications from geoelectrical, hydrochemical, modelling and statistical analyses are summarised in the larger context of a regional groundwater system with fresh- and seawater dominated sub-systems in order to outline dominant processes that lead to the observed dynamics. An outlook of possible future research for better understanding of such systems is provided at the end of this chapter.

\subsection{A conceptual model of fresh- and saltwater dynamics in the shallow groundwater aquifer at the Kapiti Coast}

A large part of hydrogeological information about the study area comes from external sources such as groundwater modelling studies and monitoring reports on behalf of the regional or local councils (e.g. Hughes, 1998; Jones and Baker, 2005; Jones and Gyopari, 2005; Tidswell, 2009; URS, 2010; Tidswell et al., 2012; Utting, 2013; NIWA, 2014; de Lange, 2014; Gyopari et al., 2014), drill log and pumping information (e.g. Jones, 2002; Brown, 2003; URS, 2003), and research projects (e.g. Wilson, 2003; Watts and Hawke, 2003; Welch, 2004; Wilson et al., 2006; Ewig, 2009; Morrow et al., 2010; Allen, 2010). In the course of this PhD project, additional structural (i.e. lithology) and temporal (i.e. behaviour, periods) information within the first $500 \mathrm{~m}$ from the shoreline at the Kapiti Coast was obtained by primarily analysing DC resistivity monitoring data. This is complemented with in situ point measurements of hydrochemical 
measurements to assess the quality of the geoelectrical information and obtain an independent temporal measure of seawater intrusion potential at the field site. Figure 7.1 shows a conceptual model of the shallow coastal aquifer which integrates lithological information from the above sources with findings of this thesis. The processes as inferred from temporal observations made in the framework of this project are discussed in the following outlining the story of the groundwater in the field area. This is the main text body. Embedded in this, stated in italics and numbered with $\mathrm{C} 1, \mathrm{C} 2$, etc., are the conclusions that can be drawn from the $\mathrm{PhD}$ project.

Imagine a rain drop that falls in the hill slopes of the Tararua Range. It has two options to end as surface runoff possibly ending up in a surface water body (e.g. stream, wetland or lake), or infiltrating the ground (e.g. through alluvial fans) and contribute to groundwater recharge. In the latter case it will, together with billions of other rain drops, make up the gravitationally driven groundwater flow in the Q2 aquifer close to the south-eastern margins of the Kapiti Coast. With a hydraulic conductivity of 10-50 m/day (Gyopari et al., 2014) the groundwater migrates towards the coast with 4-20 cm/day (Appendix B), where the geologic material changes gradually into the Holocene sands of the shallow coastal aquifer (Q1). Due to the generally finer grained nature of this medium, the groundwater flow is reduced to only about $1.5 \mathrm{~cm} /$ day (Appendix B). Where this happens, groundwater storage is increased in areas with lower hydraulic conductivity, whereas high-conductivity areas are evacuated more rapidly (Harman and Sivapalan, 2009). A couple of groundwater-surface water exchange processes happen within these two aquifer zones that can lead to either groundwater recharge or discharge. In the upper part ( $>1 \mathrm{~km}$ away from the coast) of the streams (i.e. Waikanae River, Mangaone stream) a downward flow due to the coarser material of the Q2 aquifer occurs and surface water infiltrates the aquifer at rates of 50-550 1/s (Welch, 2004; Gyopari et al., 2014) depending on location. There is also minor downward seepage $(0.05 \mathrm{~m} /$ day (Gyopari et al., 2014)) of groundwater between the Q2 and lower lying aquifer portions observed in this part.

Coming closer to the coast ( $<1 \mathrm{~km}$ from the shoreline) and into the Holocene sand, groundwater is the major factor of wetland re- and discharge. In summer months this is important in terms of wetland evaporation, which can be a significant sink of groundwater during this time of the year (Allen, 2010). The Q1 aquifer is very heterogeneous due to its deposition 


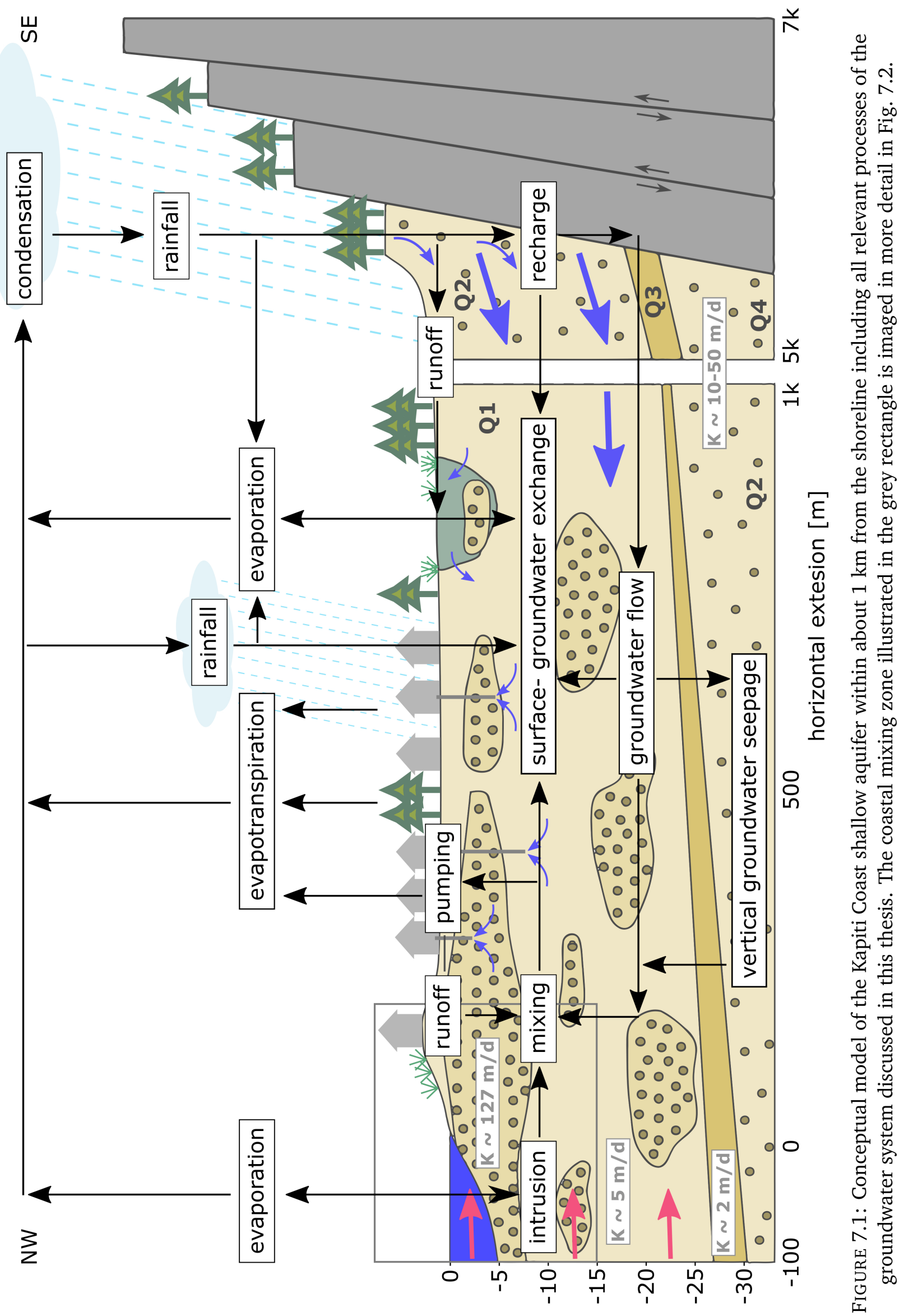

[ils.e.m] иопреләр 
history of post-glacial erosion and dune sands sedimentation with intervals of reworked river gravel deposits. This leads to varying hydraulic conductivity (2-56 m/day (Gyopari et al., 2014 and Section 6.4.3)) and therefore heterogeneous groundwater flow of approximately $1.5 \mathrm{~cm} /$ day in the Q1 bulk aquifer and $22 \mathrm{~cm}$ /day in gravel lenses within this aquifer (Appendix B). Due to the low-flow fine grained sands the hydraulic gradient leads to an upward flow of groundwater close to the coast, which recharges the rivers and streams of the area with about 150-250 1/s (Welch, 2004; Gyopari et al., 2014). This goes along with slow upward seepage of approximately $0.05 \mathrm{~m} /$ day (Gyopari et al., 2014) from lower lying aquifer units.

Surface- and groundwater exchange at the coast involves three main processes:

1. Evaporation/evapotranspiration from plants, irrigation and runoff,

2. pumping of groundwater from bores, and

3. local rainfall (events) recharge.

While (1) mainly removes water from the ground during elevated surface temperatures in summer, (2) can lead to surface runoff and re-circulate groundwater back into the aquifer (i.e. when used for irrigation or cleaning) all year round. However, pumping from the shallow aquifer appears to be increased during summer months when groundwater is widely used for private garden irrigation in the field area. Hence, bore extraction and therefore evapotranspiration from irrigation and plant evapotranspiration is possibly much higher during summer season.

C1: Local rainfall events (i.e. $>5 \mathrm{~mm} / \mathrm{h}$ ) have a short term influence on groundwater recharge in the near-surface (unsaturated and semi-saturated) parts of the coastal shallow aquifer.

For example, in dry summer months such events can lead to short term dissolution of crystallised minerals from the sediment matrix and locally increase groundwater mineralisation for a couple of days. In urban areas rainfall runoff is decreased by about $30 \%$ as compared to natural environments (Watts and Hawke, 2003) due to man-made barriers between the surface and the ground such as pavement, drainage channel system and buildings, which reduce natural runoff and infiltration by channeling the water artificially. 
C2: Anthropogenic structures lead to a changed recharge/discharge pattern in urban areas and observed opposite apparent seasonal aquifer resistivity change in zones of high hydraulic conductivity (i.e. where assumed seawater intrusion is largest).

Although, closer to the coast, the groundwater in these hydraulically more conductive lithological units clearly shows an increase in seawater typical elements and element behaviour (i.e. strontium, lithium, sodium depletion along with calcium enrichment) indicating increased seawater intrusion during summer, the overall electrical conductivity decreases.

C3: Findings from bore water hydrochemistry in different seasons indicate that a high total dissolve solids (TDS) content cannot be automatically tied to seawater intrusion. In terms of aquifer characterisation with regard to seawater intrusion it is important to take the complete groundwater chemistry into account.

Enhanced evapotranspiration, bore pumping and associated de-saturation of the gravelly sand lenses leads to crystallisation of minerals into the pore matrix and removes therefore minerals from the groundwater. This is reversed when freshwater flow increases in winter and saturation dissolves these minerals leading to an observed increase in groundwater conductivity. Saturated parts of the aquifer with lower hydraulic conductivity (i.e. fine grained sands) and areas with less urbanisation and man-made structures show the hypothesised resistivity changes in terms of seasonal behaviour (see Fig. 1.2).

C4: From the relative position of the saline interface with respect to the dunes along the rural profile N1 in the Waikanae groundwater zone and comparison between this and past studies in the Te Horo groundwater zone there are strong correlations between increased seawater intrusion and increased urbanisation.

C5: Because seawater pressure in a seasonal sense does not change significantly within a year (Bell et al., 2000), from seasonal resistivity and hydrochemistry monitoring the main controlling factor for seasonal seawater intrusion is found to be the available freshwater pressure (i.e. freshwater recharge-discharge relationships).

Within at least $200 \mathrm{~m}$ from the shoreline the shallow aquifer is additionally influenced by tidal forces. In this area, lithological (and therefore hydraulic) heterogeneity leads to preferential flow paths in the ground which are potential pathways for seawater to intrude the aquifer 
further inland. A zoom of the conceptual model for this zone is illustrated in Fig. 7.2 and the most important processes associated with it are outlined. Seawater is forced up and down the shore within a diurnal tidal cycle covering more or less area of the beach face (i.e. termed the wave run up zone). Part of this water evaporates directly at the surface, but part of the seawater infiltrates through the sand and into the aquifer. A process that also takes place below the water surface where the constant pressure landwards forces the seawater to intrude into the sedimentary matrix. Where seawater and freshwater meet, the fluids mix and internal aquifer circulation driven by density and hydraulic conductivity differences determines how salt- and freshwater flows within this zone.

C6: It is the mixing zone where the main temporal changes within a tidal time frame are associated with. Although aquifer resistivity changes reverse throughout a semi-diurnal monitoring window, the period of these changes are associated with groundwater-rather than tidal dynamics.

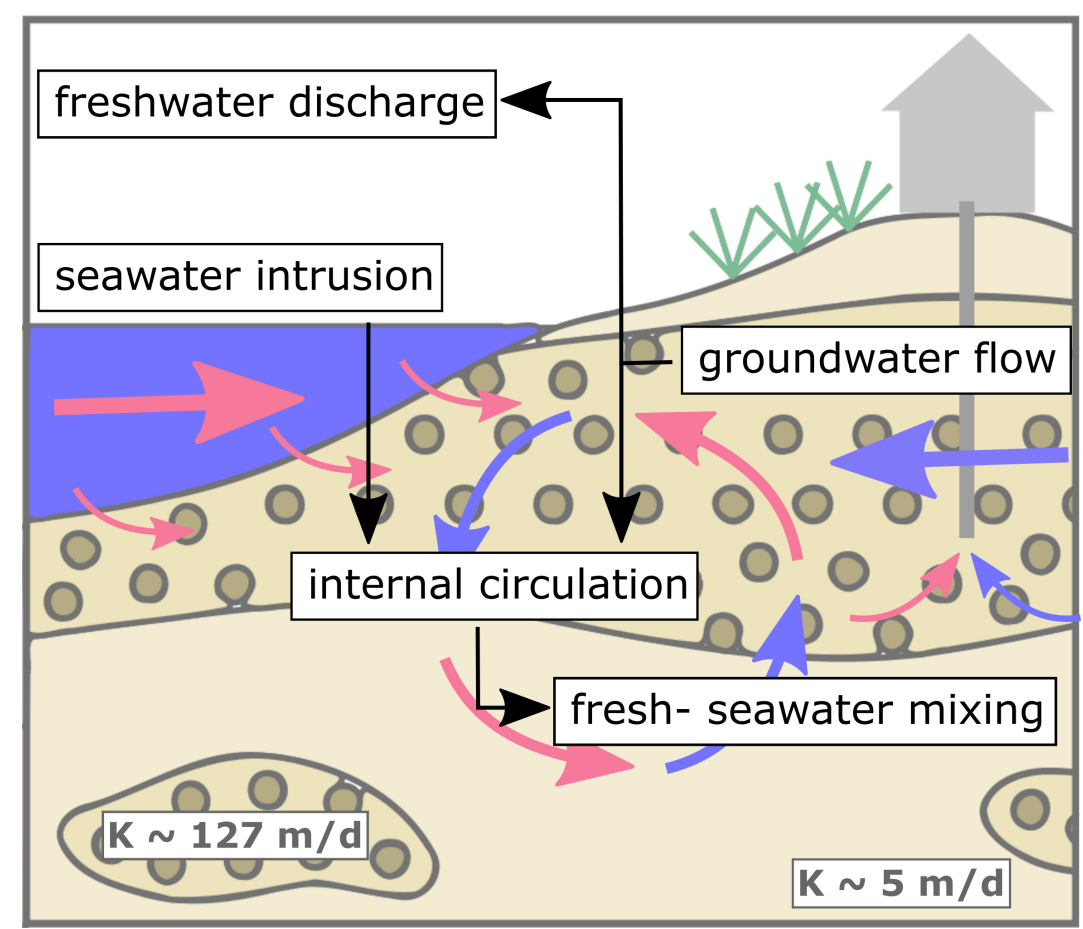

FIGURE 7.2: Zoom into the mixing zone of the shallow coastal aquifer conceptual model in Fig. 7.1 showing the relevant processes that are taking place in this zone.

Technically, DTW analysis of resistivity time series constrained with hydrological information can help to determine preferential flow paths for seawater intrusion in the coastal aquifer. In a seasonal time frame, seawater intrusion into these preferential flow paths is largely controlled 
by hydrogeology, but also to a large amount by freshwater flow in form of effective recharge from continuous rainfall.

C7: Increased continuous rainfall and therefore increased general freshwater flow towards the coast (i.e. in winter) leads to a significantly lowered amplitude in tidally influenced seawater-freshwater mixing within the shallow coastal aquifer.

Freshwater discharge to the sea is assumingly increased during these periods. Groundwater pumping from shallow (and possibly deeper) bores extracts freshwater from the aquifer. If the amount of pumped groundwater is larger than the rate of freshwater recharge, this lowers the freshwater pressure and allows more seawater to intrude further distances within the aquifer. In the worst case, seawater will eventually enter bores and wells close to the coast making the water undrinkable (i.e. $<1 \%$ seawater mixing can already render it unfit for drinking (Werner et al., 2013)). The velocity of the seawater mixing front propagation is a crucial factor for this to happen.

C8: Horizontal flow within the hydraulically more conductive gravelly sand (80-150 $\mathrm{cm} /$ day), as estimated from resistivity contour line movement, is dominant over propagation velocity within hydraulically less conductive fine grained sands $(1-5 \mathrm{~cm} /$ day) and vertical groundwater flow. Mixing of seawater therefore is much more pronounced within the gravelly sand lenses/layers and bores tapping into such zones which are directly connected to the coastline have a much larger potential of experiencing seawater contamination.

Periodic sea storms and surges that flush the shoreline above the mean high tide mark introduce seawater seepage from the surface over an extended distance inland than is normally the case. This can, depending on connectivity to hydraulically more conductive lenses within the Holocene aquifer, additionally lead to increased seawater intrusion over larger distances.

In this freshwater driven system, the rain drop that initially infiltrated the aquifer in the foothills of the Tararua Range will eventually evaporate again (may that be from a wetland, river, irrigation spray, from the soil, plants or the sea surface) and close the water cycle by condensing with several billion other water drops into a cloud. 


\subsection{Project Summary}

This project has clearly demonstrated that the DC resistivity method is well capable of imaging the saline interface and suitable to monitor salinity changes of the pore fluid in a shallow coastal Holocene sand and gravel aquifer within both, seasonal and tidal time frames. Furthermore, it was possible to show that tides do not have a measurable influence on the resistivity changes observed on resistivity surveys using a Wenner- $\alpha$ array and a 5-m minimum spacing, but salinity changes can be observed in resistivity measurements repeated over a few hours within a day using a 2-m minimum spacing for the same array type. These changes, though reflecting changed seawater mixing behaviour within a semi-diurnal time window, are likely driven by freshwater dynamics rather than tidal forcing. The source of the 18-20 hour period, which is shared between the resistivity and groundwater level changes, could not be determined with the available data, however. In an attempt to use dynamic time warping for similarity measure between time series, parts of the investigated aquifer could be tied to be influenced by groundwater and others were identified to be closer related to the tidal signal. The latter coincided with the gravel layer for which a larger hydraulic conductivity was calculated as compared to the one found for the bulk aquifer.

Combination of geoelectrical and hydrochemical measurements leads to better constrained geoelectrical model interpretation and allows to more safely extrapolate hydrochemical point measurements. Thereby unexpected observations could be put into perspective and explain that the opposite temporal behaviour of resistivity changes in a seasonal sense does not necessarily need to mean that there is a large time lag existent, but rather that intrusion dynamics are masked by other processes. Area development (urbanisation) and extensive anthropogenic influence of the natural setting has a demonstrated influence on the observed temporal behaviour of resistivity and hydrochemical parameters. In addition, resistivity and hydrochemical data acquired for this project and compared to older studies strongly indicate a link between increased anthropogenic groundwater take and increased seawater intrusion in the study area.

Generally, the shallow coastal aquifer geology was defined in greater detail thanks to the geoelectrical profiles, agreeing well with bore core information available. A hydraulically more conductive gravel layer within a hydraulically low conductive unit was found to act as 
a preferential flow path for seawater intrusion at the field location. All data analysed in the course of this $\mathrm{PhD}$ project strongly suggest that the main driving force for seawater intrusion into the shallow coastal aquifer under investigation is the availability of freshwater at any time. Therefore, the main hypothesis as outlined in Section 1.4 could be confirmed.

\subsection{Outlook}

This project applied geoelectrical and hydrochemical monitoring to discover and better understand the above outlined processes. By studying correlations between findings from both disciplines and analysing time series additionally with statistical methods a broad understanding of seawater intrusion processes into shallow sandy coastal aquifers could be demonstrated. This can be applied for future decision making in sustainable groundwater management and policy applications. In the following shortcomings and proposed further work is suggested with regard to advances in seawater intrusion monitoring and associated processes.

\subsubsection{Geoelectrical methods}

This project clearly demonstrated the applicability of the surface-based DC resistivity method to study the saline interface and also, by means of time-lapse monitoring, seawater intrusion processes. In terms of resolution, a smaller electrode spacing (i.e. 2-m minimum) was clearly beneficial for studying short-term tidal fluctuations, which could not be resolved with the larger 5-m minimum spacing. However, surface geoelectrics has the disadvantage of losing resolution rapidly with increasing distance away from the current source (i.e. increasing depth). Therefore, future seawater intrusion monitoring studies that apply the geoelectrical method should include:

$\times$ Borehole and/or cross-borehole measurements within the seawater-freshwater mixing zone, but also further away from the coastline in order to increase vertical resolution and enable the possibility to study the small resistivity changes (i.e. 10-50 $\Omega \mathrm{m}$ ) within different time frames better.

It was attempted to do such measurements as the main part of this project initially. However, due to permit and cost problems this was prevented from occurring. 
$\times$ Automated monitoring with fixed arrays to decrease the likelihood of varying electrode positions between time steps and decrease the sampling interval.

$\times$ Depending on the underground geology, additional geophysical methods such as refraction/reflection seismics, ground penetrating radar (GPR) and electromagnetic (EM) methods should be applied along the geoelectrical transects in order to provide lithologically better constrained inversion models (i.e. by joint inverting these methods). This was not possible at the field location chosen for this project as the attenuation in the loose sands was too high to get reliable signals from seismics or GPR. EM methods were unsuitable due to the electromagnetic noise in the area (e.g. surface and subsurface wires).

\subsubsection{Hydrochemical methods}

From three hydrochemical analyses of groundwater from 18 shallow bores seasonal changes in groundwater chemistry could be determined and seawater intrusion potential within different hydrogeological units was obtained within a seasonal time frame. However, there are a few facotrs that should be taken into account for future hydrochemical studies. These include:

$\times$ A larger range of vertical depth penetration for the chosen sampling bores would be beneficial (depending on availability). This is especially recommended when using hydrochemistry in conjunction with the geoelectrical (or another geophysical) method as it allows significantly improved constraints on the inversion results.

$\times$ The sampling interval should be decreased in order to make sure that measurements show a continuous change and therefore decrease the possibility of such point measurements being a possible exception/measurement error. This would imply the use of a reasonably cheap analysis method, however.

$\times$ As seawater contains chloride to a substantial part along with other seawater indicating anions (e.g. sulfate, bromide) it would be beneficial to use an additional groundwater chemistry analysis method (e.g. chromatography) as well as the applied IC-PMS method as the latter is not sufficiently sensitive for some anions to be determined. This would 
allow investigation of important additional seawater intrusion specific relationships such as using Piper plots to determine the source of a groundwater sample.

$\times$ Analysis of a sample of the seawater at the field location for the same elements as the groundwater samples are analysed for should be aspired to. Thus, a suitable dilution or technique for the high-concentration seawater analysis needs to be found.

$\times$ Sensors used to measure in situ parameters such as electric conductivity, total dissolved solids, $\mathrm{pH}$ and dissolved oxygen in the field should be calibrated with the same standards before every sampling is carried out. This should ensure comparability between individual samplings. Furthermore, attaching a sterilised/rinsed hose to the sampling tap would make sure false dissolved oxygen readings are not introduced and decrease the possibility of chemical reactions of elements in the groundwater directly before sampling.

\subsubsection{Time series methods}

It has been demonstrated that time series obtained from geoelectrical monitoring data can be successfully analysed for their temporal behaviour with a range of time series analysis techniques. Resistivity ratios showed good agreement with dynamic time warping (DTW) analysis in outlining parts of the aquifer which react differently. Autocovariance of a long ( $>12$ hours) geoelectrical time series and Fourier transformation of groundwater stage monitoring data from a well allowed determination of the dominant period for changes in subsurface flow. However, despite using these methods successfully with the present data, there is room for improvement in future applications.

$\times$ The DTW analysis showed that optimal warping cost (OWC) values obtained are highly depending on the length of the monitoring window relative to the period of the subsurface signal, especially where this period differs from that of the tidal stage. Additionally, the placement of this monitoring window with respect to the relative temporal relationships between the signals being compared is important in order to obtain a correct measure of time lag. Future research that applies this method to geoelectrical signals should carefully choose the monitoring period according to the resistivity signal period and is recommended to be at least twice as long as this period. 
$\times$ In general, tidal monitoring time series should aim to be longer than the tidal stage period in order to obtain well estimated periods for the resistivity signal through autocovariance. Such longer time series could be achieved with fixed monitoring systems. To increase temporal resolution a more frequent rate of sampling should be used. However, with acquisition times of roughly $15-20 \mathrm{~min}$ for a $78 \mathrm{~m}$ long 2-m initial electrode spaced Wenner- $\alpha$ array the sample interval can realistically only be approximately halved.

$\times$ Further research in applicability of statistical methods to geoelectrical (and other geophysical) time series is highly recommended in order to obtain a robust assessment method to investigate time lags between oscillating signals. DTW could be a promising tool, but this thesis has shown that the method clearly requires more work before it can be regarded a reliable way of obtaining a signal similarity measure.

\subsubsection{Other recommendations}

It should be the goal to carry out more interdisciplinary research that takes advantage of several well established methods from different fields in order to overcome shortcomings of each one of the methods used and therefore substantially improve our knowledge about seawater intrusion dynamics in coastal aquifers. Furthermore, such studies should be applied to a variety of geologically different environments, as small differences in hydrogeological properties can lead to varying temporal observations in seawater intrusion characterisation as could be seen from comparisons between the two different field sites discussed in this thesis.

And to say it with the words of Custodio (1987): "In dealing with resource and water problems, the subsystem should be understood within the framework of the larger resource system." 


\section{Bibliography}

Abarca, E., Karam, H., Hemond, H. F., and Harvey, C. F. (2013). Transient groundwater dynamics in a coastal aquifer: The effects of tides, the lunar cycle and the beach profile. Water Resources Research, 49:2473-2488.

Acworth, R. I. and Dasey, G. R. (2003). Mapping of the hyperoheic zone around a tidal creek using a combination of borehole logging, borehole electrical tomography and cross-creek electrical imaging, New South Wales, Australia. Hydrogeology Journal, 11:368-377.

Aitchinson-Earl, P., Ettema, M., Hanson, C., Hayward, S., Larking, R., Sanders, R., Scott, D., and Veltman, A. (2003). Coastal aquifer saltwater intrusion assessment guidelines. Technical report, Environment Canterbury.

Albouy, Y., Andrieux, P., Rakotondrasoa, G., Ritz, M., Descloitres, M., Join, J.-L., and Rasolomanana, E. (2001). Mapping Coastal Aquifers by Joint Inversion of DC and TEM Soundings - Three Case Histories. Ground Water, 39(1):87-97.

Allen, W. C. (2010). Hydrological characteristics of the Te Hapua wetland complex: the potential influence of groundwater level, bore abstraction and climate change on wetland surface water levels. Master's thesis, Victoria University of Wellington.

Archie, G. E. (1942). The Electrical Resistivity Log as an Aid in Determining Some Reservoir Characteristics. Technical report, Society of Petroleum Engineers.

Ashman, K. M., Bird, C. M., and Zepf, S. E. (1994). Detecting bimodality in astronomical datasets. Astronomical Journal, 108:2348-2361.

Ataie-Ashtiani, B., Volker, R. E., and Lockington, D. A. (1999). Tidal effects on sea water intrusion in unconfined aquifers. Journal of Hydrology, 216:17-31. 
Ayolabi, E. A., Folorunso, E. F., Odukoya, A. M., and Adeniran, A. E. (2013). Mapping saline water intrusion into the coastal aquifer with geophysical and geochemical techniques: the University of Lagos campus case (Nigeria). SpringerPlus, 3(433).

Badon Ghyben, W. (1889). Nota in verband met de voorgenomen putboring nabij Amsterdam (Notes on the probable results of the proposed well drilling near Amsterdam). Tijdschrift het koninklijk Instituut voor Ingenieurs, The Hague, pages 8-22.

Barlow, P. M. (2003). Ground Water in Freshwater-Saltwater Environments of the Atlantic Coast. U.S. Geological Survey Circular 1262, U.S. Geological Survey.

Bauer, P., Supper, R., Zimmermann, S., and Kinzelbach, W. (2006). Geoelectrical imaging of groundwater salinization in the Okavango Delta, Botswana. Journal of Applied Geophysics, 60:126-141.

Beaujean, J., Nguyen, F., Kemna, A., Antonsson, A., and Engesgaard, P. (2014). Calibration of seawater intrusion models: Inverse parameter estimation using surface electrical resistivity tomography and borehole data. Water Resources Research, 50:6828-6849.

Beck, W. and Hanshaw, B. B. (1970). Comparison of chemical hydrology of the carbonate peninsulas of Florida and Yucatan. Journal of Hydrology, 10(4):330-368.

Bekesi, G. (1996). Nitrate-Nitrogen in Horowhenua Groundwater. Technical report, Manawatu Wanganui Regional Council.

Bell, R. G., Goring, D. G., and de Lange, W. P. (2000). Sea-level change and storm surges in the context of climate change. IPENZ Transactions, 27(1):10 pp.

Binley, A., Hubbard, S. S., Huisman, J. A., Revil, A., Robinson, D. A., Singha, K., and Slater, L. D. (2015). The emergence of hydrogeophysics for improved understanding of subsurface processes over multiple scales. Water Resources Research, 51:3837-3866.

British Geological Survey (2003). Water Quality Fact Sheet: Managnese. Online.

Brown, L. (2003). Te Horo Beach Groundwater Investigation Bores. Technical report, Resource Investigations Greater Wellington Regional Council. 
Burnett, W. C., Aggarwal, P. K., Aureli, A., Bokuniewicz, H., Cable, J. E., Charette, M. A., Kontar, E., Krupa, S., Kulkarni, K. M., Loveless, A., Moore, W. S., Oberdorfer, J. A., Oliveira, J., Ozyurt, N., Povinec, P., Privitera, A. M. G., Rajar, R., Ramessur, R. T., Scholten, J., Stieglitz, T., Taniguchi, M., and Turner, J. V. (2006). Quantifying submarine groundwater discharge in the coastal zone via mulitple methods. Science of the Total Environment, 367(2-3):498-543.

Callander, P., Lough, H., and Steffens, C. (2011). New Zealand Guidelines for the Monitoring and Management of Sea Water Intrusion Risks on Groundwater. Technical report, Envirolink Project 420-NRLC50.

Chester, R. (2000). Marine Geochemistry. Blackwell Science, Oxford UK, 2nd edition.

Comte, J.-C. and Banton, O. (2007). Cross-validation of geo-electrical and hydrogeological models to evaluate seawater intrusion in coastal aquifers. Geophysical Research Letters, 34(L10402).

Cooper, H. H. J., Kohout, F. A., Henry, H. R., and Glober, R. E. (1964). Sea water in coastal aquifers. U.S. Geological Survey Water-Supply Paper 163-C.

Coscia, I. (2012). Time-Varying Electrical Properties of Infiltrating River Water as a Natural Tracer for Investigating Flow and Transport in an Aquifer Through Time-Lapse Crosshole ERT. PhD thesis, ETH Zurich.

Cotecchia, V. (1977). Direct and laboratory observations of the mixing phenomena of fresh and salt water in coastal groundwater: particular case of fractured media. Symposium on Hydrodynamic Diffusion and Dispersion in Porous Media, Pavia.

Custodio, E. (1967). Hydrogeochemical studies in the Llobregat delta, Barcelona, Spain. General Assembly of Bern. Int. Assoc. Scientific Hydrology, 62:135-155.

Custodio, E. (1987). Salt-fresh water interrelationships under natural conditions. In Custodio, E. and Bruggeman, G. A., editors, Studies and reports in hydrology: groundwater problems in coastal areas, pages 14-96. UNESCO, Paris.

De Franco, R., Biella, G., Tosi, L., Teatini, P., Lozej, A., Chiozzotto, B., Giada, M., Rizzetto, F., Claude, C., Mayer, A., Bassan, V., and Gasparetto-Stori, G. (2009). Monitoring the saltwater intrusion by time lapse electrical resistivity tomography: The Chioggia test site (Venice Lagoon, Italy). Journal of Applied Geophysics, 69:117-130. 
de Lange, W. (2014). Kapiti Coast coastal hazard assessment. Technical report, Kapiti Coast District Council Report.

Deceuster, J., Etienne, A., Robert, T., Nguyen, F., and Kaufmann, O. (2014). A modified DOIbased method to statistically estimate the depth of investigation of dc resistivity surveys. Journal of Applied Geophysics, 103:172-185.

deGroot-Hedlin, C. and Constable, S. (1990). Occam's Inversion to generate smooth, twodimensional models from magnetotelluric data. Geophysics, 55(12):1613-1624.

Diebold, F. X., Kilian, L., and Nerlove, M. (2006). Time Series Analysis. In Blume, L. and Durlauf, S., editors, The New Palgrave Dictionary of Economics, Second Edition. Macmillan, London.

Erskine, A. D. (1991). The Effect of Tidal Fluctuation on a Coastal Aquifer in the UK. Ground Water, 29(4):556-562.

Ewig, E. (2009). Lithospheric shortening and ductile deformation in a back-arc setting: South Wanganui Basin, New Zealand. PhD thesis, Victoria University of Wellington.

Ferris, J. G. (1951). Cyclic fluctuations of water level as a basis for determining aquifer transmissibility. International Association of Hydrological Sciences Journal, 2:148-155.

Fraser, K., Law, M., and Avanidou, D. (2014). Annual Waikanae Borefiled Report 2013/14 (Consent WGN130103 [31992]). Technical report, CH2M Beca Limited.

Fulton, J. W., Koerkle, E. H., McAuley, S. D., Hoffman, S. A., and Zarr, L. F. (2005). Hydrogeologic Setting and Conceptual Hydrologic Model of the Spring Creek Basin, Center County, Pennsylvania, June 2005. Scientific investiagions report 2005-5091, U.S. Department of the Interior and U.S. Geological Survey.

Glover, R. E. (1959). The pattern of fresh-water flow in a coastal aquifer. Journal of Geophysical Research, 64(4):457-459.

GNS Science NZ (2016). Water Sure - groundwater science. Online information leaflet.

Gunawardhana, L. N. and Kazama, S. (2009). Tidal effects on aquifer thermal regime: An analytical solution for coastal ecosystem management. Journal of Hydrology, 377:377-390. 
Gyopari, M., Mzila, D., and Hughes, B. (2014). Kapiti Coast groundwater resource investigation. Catchment hydrogeology and modelling report, Greater Wellington Regional Council.

Hale, D. (2013). Dynamic warping of seismic images. Center for Wave Phenomena, Colorado School of Mines (USA).

Han, D., Post, V. E. A., and Song, X. (2015). Groundwater salinization processes and reversibility of seawater intrusion in coastal carbonate aquifers. Journal of Hydrology, 531:1067-1080.

Harman, C. and Sivapalan, M. (2009). Effect of hydraulic conductivity variability on hillslopescale shallow subsurface flow response and storage-discharge relations. Water Resources Research, 45(1):W01421.

Heath, R. C. (1983). Basic Ground-Water Hydrology. U.S. Geological Survey Water-Supply Paper 2220, 86p. U.S. Geological Survey.

Heiss, J. W. and Michael, H. A. (2014). Saltwater-freshwater mixing dynamics in a sandy beach aquifer over tidal, spring-neap, and seasonal cycles. Water Resources Research, 50:6747-6766.

Heiss, J. W., Puleo, J. A., Ullman, W. J., and Michael, H. A. (2015). Coupled surface-subsurface hydrologic measurements reveal infiltration, recharge, and discharge dynamics across the swash zone of a sandy beach. Water Resources Research, 51:8834-8853.

Hele-Shaw, H. S. (1897). Experiments on the nature of surface resistance in pipes and on ships. Transactions Institute Naval Architects, 39:145-146.

Hele-Shaw, H. S. (1899). Stramline motion of a viscous film. British Association for the Advancement of Science, 68th Meeting, 136:136-142.

Hem, J. D. (1985). Study and Interpretation of the Chemical Characteristics of Natural Water. Technical report, U.S. Geological Survey Water-Supply Paper 2254.

Hem, J. D. (1989). Study and Interpretation of the Chemical Characteristics of Natural Water (3rd edition). U.S. Geological Survey Water-Supply Paper 2254.

Henderson, R. D., Day-Lewis, F. D., Abarca, E., Harvey, C. F., Karam, H. N., Liu, L., and Jr., J. W. L. (2010). Marine electrical resistivity imaging of submarine groundwater discharge: 
sensitivity analysis and application in Waquoit Bay, Massachusetts, USA. Hydrogeology Journal, 18:173-185.

Henry, H. R. (1964). Interfaces between salt water and fresh water in coastal aquifers. In et al., H. H. C., editor, Sea Water in Coastal Aquifers, pages 35-69. U. S. Geological Survey.

Hermans, T., Vandenbohede, A., Lebbe, L., Martin, R., Kemna, A., Beaujean, J., and Nguyen, F. (2012). Imaging artificial salt water infiltration using electrical resistivity tomography constrained by geostatistical data. Journal of Hydrology, 438-439:168-180.

Herzberg, A. (1901). Die Wasserversorgung einiger Nordseebaeder (The water supply on parts of the North Sea coast). Journal fuer Gasbeleuchtung und Wasserversorgung, Muenchen, 44 $45: 815-819,842-844$.

Hilbich, C., Marescot, L., Hauck, C., Loke, M. H., and Maeusebacher, R. (2009). Applicability of Electrical Resistivity Tomography Monitoring to Coarse Blocky and Ice-rich Permafrost Landforms. Permafrost and Periglacial Processes, 20(3):269-284.

Hughes, B. (1998). Te Horo Beach Groundwater Quality Investigation. WRC Groundwater Report WRC/RINV-T-98/10, Wellington Regional Council.

Ibanez, J. G., Hernandez-Esparza, M., Doria-Serrano, C., Fregoso-Infante, A., and Mohan Singh, M. (2007). Environmental Chemistry Fundamentals. Springer Science and Business Media LLC.

Inouchi, K., Kishi, Y., and Kakinuma, T. (1990). The Motion of Coastal Groundwater in Response to the Tide. Journal of Hydrology, 115:165-191.

Ivkovic, K. M., Marshall, S. K., Morgan, L. K., Werner, A. D., Carey, H., Cook, S., Sundaram, B., Norman, R., Wallace, L., Caruana, L., Dixon-Jain, P., and Simon, D. (2012). Nationalscale vulnerability assessment of seawater intrusion: summary report. Waterlines report, National Water Commission, Canberra.

Jacob, C. E. (1950). Flow of ground water. In Rouse, H., editor, Engineering hydraulics, pages 321-386. Wiley, Hoboken, NJ. 
Jeanson, B. and Dufort, J. (1970). Etude sur modele physique des conditions de pollution des nappes cotieres par l'eau salée lors de la production. Bulletin BRGM, 2nd Series, III(3):98-148.

Johnson, A. I. (1967). Specific yield - compilation of specific yields for various materials. U.S. Geological Survey Water-Supply Paper 1662-D.

Johnson, T. C., Slater, L. D., Ntarlagiannis, D., Day-Lewis, F. D., and Elwaseif, M. (2012). Monitoring groundwater-surface water interaction using time-series and time-frequency analysis of transient three-dimensional electrical resistivity changes. Water Resources Research, 48:W07506, 13 pp.

Jones, A. (2002). Sims Road Pump Tests Mourie Property Te Horo. Technical Report WRC/RI-T02/05, Wellington Regional Council.

Jones, A. and Baker, T. (2005). Groundwater monitoring technical report. GWRC Groundwater Technical Report 1, Greater Wellington Regional Council.

Jones, A. and Gyopari, M. (2005). Investigating the sustainable use of shallow gorundwater on the Kapiti Coast. Technical report, Greater Wellington Resource Investigations Department.

Kim, J.-H., Yi, M.-J., Park, S.-G., and Kim, J. G. (2009). 4-D inversion of DC resistivity monitoring data acquired over a dynamically changing earth model. Journal of Applied Geophysics, 68:522-532.

Kim, K.-Y., Seong, H., Kim, T., Park, K.-H., Woo, N.-C., Park, Y.-S., Koh, G.-W., and Park, W.-B. (2006). Tidal effects on variations of fresh-saltwater interface and groundwater flow in a multilayered coastal aquifer on a volcanic island (Jeju Island, Korea). Journal of Hydrology, 330:525-542.

Kirsch, R. (2006). Groundwater Geophysics. Springer Berlin Heidelberg New York.

Knoedel, K., Krummel, H., and Lange, G. (2005). Geophysik. Springer Berlin Heidelberg New York, 2nd edition.

Kobierska, F., Jonas, T., Griessinger, N., Hauck, C., Huxol, S., and Bernasconi, S. M. (2015). A multi-method field experiment to determine local groundwater flow in a glaicer forefield. Hydrological Processes, 29(6):817-827. 
Kohout, F. A. (1964). The flow of fresh water and salt water in the Biscayne aquifer of the Miami Area, Florida. Sea Water in Coastal Aquifers. U.S. Geological Survey Water-Supply Paper 1613-C, pages C12-C32.

Kuan, W. K., Jin, G., Xin, P., Robinson, C., Gibbes, B., and Li, L. (2012). Tidal influence on seawater intruison in unconfined coastal aquifers. Water Resources Research, 48:W02502, $11 \mathrm{pp}$.

LaBrecque, D. J. and Yang, X. (2000). Difference Inversion of ERT Data: A Fast Inversion Method for 3-D In-Situ Monitoring. Proceedings of the Symposium of the Application of Geophysics to Engineering and Environmental Problems, pages 907-914.

Lagudu, S., Rao, V. V. S. G., Prasad, P. R., and Sarma, V. S. (2013). Use of geophysical and hydrochemical tools to investigate seawater intrusion in coastal alluvial aquifer, Andhra Pradesh, India. In Wetzelhuetter, C., editor, Groundwater in the Coastal Zones of Asia-Pacific, pages 49-65. Springer Dordrecht, The Netherlands.

Levanon, E., Shalev, E., Yechieli, Y., and Gvirtzman, H. (2016). Fluctuations of fresh-saline water interface of water table induced by sea tides in unconfined aquifers. Advances in Water Resources, 96:34-42.

Lines, L. R. and Treitel, S. (1984). Tutorial - A Review of Least-Squares Inversion and its Application to Geophysical Problems. Geophysical Prospecting, 32:159-186.

Loke, M. H. (2004). Tutorial: 2-D and 3-D electrical imaging surveys. Online.

Loke, M. H., Acworth, I., and Dahlin, T. (2006). A comparison of smooth and blocky inversion models in 2D electrical imaging surveys. Exploration Geophysics, 34:182-187.

Loke, M. H., Chambers, J. E., Rucker, D. F., Kuras, O., and Wilkinson, P. B. (2013). Recent developments in the direct-current geoelectrical imaging method. Journal of Applied Geophysics.

Mandal, A., Zhang, J., and Asai, K. (2011). Stable isotopic and geochemical data for inferring sources of recharge and groundwater flow on the volcanic island of Rishiri, Japan. Applied Geochemistry, 26:1741-1751. 
Marescot, L., Loke, M. H., Chapellier, D., Delaloye, R., Lambiel, C., and Reynard, E. (2003). Assessing reliability of $2 \mathrm{D}$ resistivity imaging in mountain permafrost studies using the depth of investigation index method. Near Surface Geophysics, pages 57-67.

Maurer, H. (2012). Inversion Theory for Applied Geophysics. Lecture Script ETH Zurich.

McGranahan, G., Balk, D., and Anderson, B. (2007). The rising tide: assessing the risks of climate change and human settlements in low elevation coastal zones. Environment and Urbanization, 19:17-37.

Menke, W., editor (2012). Geophysical Data Analysis: Discrete Inverse Theory (Third Edition). Academic Press, Boston, 3rd edition.

Michael, H. A., Mulligan, A. E., and Harvey, C. F. (2005). Seasonal oscillations in water exchange between aquifers and the coastal ocean. Nature Letters, 436:1145-1148.

Ministry of Health New Zealand (2008). Drinking-water Standards for New Zealand 2005 (Revised 2008). Online.

Moreau, M. and Bekele, M. (2015). Groundwater component of the Water Physical Stock Account (WPSA). Technical report, GNS Science Consultancy Report 2014/290.

Morrow, F. J. (2007). Dynamics of the saline interface of the shallow unconfined aquifer at Kapiti. Master's thesis, Victoria University of Wellington.

Morrow, F. J., Ingham, M. R., and McConchie, J. A. (2010). Monitoring of tidal influences on the saline interface using resistivity traversing and cross-borehole resistivity tomography. Journal of Hydrology, 389:69-77.

Mulligan, A. E., Evans, R. L., and Lizarralde, D. (2007). The role of paleochannels in gorundwater/seawater exchange. Journal of Hydrology, 335:313-329.

Mzila, D. (2013). Resource Consent Application WGN130103: Hydrogeology Technical Review. Resource Consent Application Review 1211068-V3, Greater Wellington Regional Council.

Nelson, D. (2002). Natural Variations in the Composition of Groundwater. Groundwater Foundation Annual Meeting. 
Nguyen, F., Kemna, A., Antonsson, A., Engesgaard, P., Kuras, O., Ogilvy, R., Gisbert, J., Jorreto, S., and Pulido-Bosch, A. (2009). Characterization of seawater intrusion using 2D electrical imaging. Near Surface Geophysics, 7:377-390.

NIWA (2014). The Climate and Weather of Wellington Region. Number 65 in NIWA Science and Technology Series. National Institute of Water and Atmospheric Research.

Ogilvy, R. D., Meldrum, P. I., Kuras, O., Wilkinson, P. B., Chambers, J. E., Sen, M., Pulido-Bosch, A., Gisbert, J., Jorreto, S., Frances, I., and Tsourlos, P. (2009). Automated monitoring of coastal aquifers with electrical resistivity tomography. Near Surface Geophysics, 7:367-375.

Oldenburg, D. W. and Li, Y. (1999). Estimating depth of investigation in dc resistivity and IP surveys. Geophysics, 2:403-416.

Osborne, A. M. (2006). Movement of water within the Waikanae Shallow Gravel Aquifer and its Interaction with the Waikanae River. Master's thesis, Victoria University of Wellington.

Oude Essink, G. H. P. (2001). Improving fresh groundwater supply - problems and solutions. Ocean and Coastal Management, 44:429-449.

Palacky, G. J. (2006). Resistivity Characteristics of Geologic Targets. In Nabighian, M. N., editor, Electromagnetic Methods in Applied Geophysics, number 3 in Investigations in Geophysics, chapter 3, pages 53-130. Society of Exploration Geophysicists, Tulsa, Oklahoma USA, 3rd edition.

Parliamentary Commissioner for the Environment (2015). Preparing New Zealand for rising seas: Certainty and Uncertainty. Online.

Pearson, K. (1929). Editorial note. Biometrika, 21:370-375.

PerkinElmer (2011). The 30-Minute Guide to ICP-MS. Technical Note ICP-Mass Spectrometry.

Perri, M. T., Cassiani, G., Gervasio, I., Deiana, R., and Binley, A. (2012). A saline tracer test monitored via both surface and cross-borehole electrical resistivity tomography: Comparison of time-lapse results. Journal of Applied Geophysics, 79:6-16.

Petitjean, F., Inglada, J., and Gancarski, P. (2012). Satellite Image Time Series Analysis Under Time Warping. IEEE Transactions on Geoscience and Remote Sensing, 50(8):3081-3095. 
Poulsen, S. E., Rasmussen, K. R., Christensen, N. B., and Christensen, S. (2010). Evaluating the salinity distribution of a shallow coastal aquifer by vertical multielectrode profiling (Denmark). Hydrogeology Journal, 18:161-171.

Raj, D. and Shaji, E. (2017). Fluoride contamination in groundwater resources of Alleppey, southern India. Geoscience Frontiers, 8:117-124.

Reynolds, J. M. (2011). An Introduction to Applied and Environmental Geophysics. John Wiley and Sons, Chichester, West Sussex UK, 2nd edition.

Riley, J. P. and Tongudai, M. (1964). The lithium content of sea water. Deep-Sea Research, 11:563-568.

Robert, T., Dessargues, A., Brouyère, S., Kaufmann, O., Hallet, V., and Nguyen, F. (2011). Assessing the contribution of electrical resistivity tomography (ERT) and self-potential (SP) methods for a water well drilling program in fractured/karstified limestones. Journal of Applied Geophysics, 75:42-53.

Robertson, B. and Stevens, L. (2007). Kapiti, Southwest, South Coasts and Wellington Harbour: risk assessment and monitoring recommendations. Technical report, Report prepared for Greater Wellington Regional Council by Wiggle Coastal Management, Nelson.

Robinson, C., Gibbes, B., Carey, H., and Li, L. (2007). Salt-freshwater dynamics in a subterranean estuary over a spring-neap tidal cycle. Journal of Geophysical Research, 112:C09007, 15 pp.

Rojas, R. (2010). Why the Normal Distribution? Online, Freie Universitaet Berlin.

Rotzoll, K., Gingerich, S. B., Jenson, J. W., and El-Kadi, A. I. (2013). Estimating hydraulic properties from tidal attenuation in the Northern Guam Lens Aquifer, territory of Guam, USA. Hydrogeology Journal, 21(3):643-654.

Rovey, C. W. and Cherkauer, D. S. (1995). Scale Dependency of Hydraulic Conductivity Measurements. Ground Water, 33(5):769-780.

Rumer, R. R. and Harleman, D. R. F. (1963). Intruded salt-water wedge in porous media. Journal of Hydraulics Division, 89 (HY6):189-220.

Sakoe, H. and Chiba, S. (1978). Dynamic programming algorithm optimization for spoken word recognition. IEEE Transactions on Acoustics, Speech and Signal Processing, 26:43-49. 
Sasaki, Y. (1992). Resolution of Resistivity Tomography Inferred from Numerical Simulation. Geophysical Prospecting, 40:453-463.

Schlumberger Water Services (2010). Diver-Suite - Water Level Loggers Product Manual.

Semkin, R. G., Jeffries, D. S., and Clair, T. A. (1994). Hydrochemical Methods and Relationships for Study of Stream Output from Small Catchments. In Moldan, B. and Cerny, J., editors, Biogeochemistry of Small Catchments: A Tool for Environmental Research, chapter 7, pages 165-188. John Wiley and Sons Ltd.

Shumway, R. H. and Stoffer, D. S. (2016). Time Series Analysis and Applications. Using the R Statistical Package. Free Dog Publishing.

Slater, L., Binley, A. M., Daily, W., and Johnson, R. (2000). Cross-hole electrical imaging of a controlled saline tracer injection. Journal of Applied Geophysics, 44:85-102.

Tadolini, T. and Tulipano, L. (1975). Measurement of chlorine, bormine and iodine content in groundwater from the Salentine peninsula (Southern Italy) in respect to the sea water encroaching the continent. 3rd International Convention on Groundwater, Palermo.

Taniguchi, M. (2002). Tidal effects on submarine groundwater discharge into the ocean. Geophysical Research Letters, 29(12):1561, 3 pp.

Thomas, N. and Callander, P. (2013). Review of Horizons Coastal Groundwater Monitoring Network. Report no. 2013/ext/1317, Horizons Regional Council.

Thomas, R. (2004). Practical Guide to ICP-MS. Marcel Dekker Inc, New York and Basel.

Tidswell, S. (2009). Kapiti Coast groundwater quality investigation, 2008. GWRC Technical Report GW/EMI-T-09/246, Greater Wellington Regional Council.

Tidswell, S., Conwell, C., and Milne, J. R. (2012). Groundwater quality in the Wellington region. State and trends. Report gw/emi-t-12/140, Greater Wellington Regional Council.

Todd, D. K. (1958). Groundwater hydrology. John Wiley and Sons, New York.

Todd, D. K. and Meyer, C. F. (1971). Hydrology and geology of the Honolulu aquifer. Proceedings of the American Society of Civil Engineers, HY2:233-356. 
Turner, I. L. and Acworth, R. I. (2004). Field Measurements of Beachface Salinity Structure using Cross-Borehole Resistivity Imaging. Journal of Coastal Research, 20:753-760.

URS (2003). Waikanae/Otaihanga Borefield Drilling Strategy. Technical report, Kapiti Coast District Council, URS New Zealand Limited.

URS (2010). Monitoring Manual Waikanae Borefield and Back-up Bores (PW1, PW5). Report 42749750/01/01, Kapiti Coast District Council.

U.S. EPA (2005). Quick Guide To Drinking Water Sample Collection. Technical report, United States Environmental Protection Agency.

Utting, M. (2013). Kapiti Coast - Revised Aquifer Testing and Groundwater Modelling. Report for Kapiti Coast District Council (KCDC) NZ1-6134419-61, CH2M Beca Limited.

Van Essen Instruments (2016). Product Manual Diver.

Voorhees, K. J., Hickey, J. C., and Klusman, R. W. (1984). Analysis of Groundwater Contamination by a New Surface Static Trapping/Mass Spectrometry Technique. Analytical Chemistry, 56(13):2602-2604.

Wagner, F. M., Moeller, M., Schmidt-Hattenberger, C., Kempka, T., and Maurer, H. (2013). Monitoring freshwater salinization in analog transport models by time-lapse electrical resistivity tomography. Journal of Applied Geophysics, 89:84-95.

Watts, L. F. and Hawke, R. M. (2003). The effects of urbanisation on hydrologic resoponse: a study of two coastal catchments. Journal of Hydrology (NZ), 42(2):125-143.

Weir, J. and Davidson, P. (2016). Wairau Aquifer Groundwater Model. Prediction of Climate Change Impacts. Report c16061, Marlborough District Council.

Welch, C. (2004). Groundwater recharge form the Waikanae River. Unpublished BSc (Hons) thesis, Victoria University of Wellington.

Werner, A. D., Bakker, M., Post, V. E. A., Vandenbohede, A., Lu, C., Ataie-Ashtiani, B., Simmons, C. T., and Barry, D. A. (2013). Seawater intrusion processes, investigation and management: Recent advances and future challenges. Advances in Water Resources, 51:3-26. 
Werner, A. D. and Lockington, D. A. (2006). Tidal impacts on riparian salinities near estuaries. Journal of Hydrology, 328:511-522.

Western, A. W., Grayson, R. B., and Costelloe, J. F. (2005). Principles of Hydrological Measurements. In Anderson, M. G., editor, Encyclopedia of Hydrological Sciences, chapter 6. John Wiley and Sons Ltd.

WHO (2011). Guidelines for drinking-water quality. World Health Organization, 4th edition.

WHO (2015). Chemical fact sheets. In Guidelines for drinking-water quality, pages 307-442. World Health Organization.

Wilkinson, P. B., Chambers, J. E., Meldrum, P. I., Gunn, D. A., Ogilvy, R. D., and Kuras, O. (2010). Predicting the movements of permanently installed electrodes on an active landslide using time-lapse geoelectrical resistivity data only. Geophysical Journal International, 183:543556.

Wilson, S. R. (2003). The Saltwater-Freshwater Interface, Te Horo Beach, Kapiti Coast. Master's thesis, Victoria University of Wellington.

Wilson, S. R., Ingham, M., and McConchie, J. A. (2006). The applicability of earth resistivity methods for saline interface definition. Journal of Hydrology, 316:301-312.

Wolf, R. E. (2005). What is ICP-MS? ... and more importantly, what can it do? U. S. Geological Survey CR/CICT.

Wood, C. and Harrington, G. A. (2015). Influence of Seasonal Variations in Sea Level on the Salinity Regime of a Coastal Groundwater-Fed Wetland. Groundwater, 53(1):90-98.

Yamamoto-Kawai, M., Carmack, E. C., McLaughlin, F. A., and Falkner, K. K. (2010). Oxygen isotope ratio, barium and salinity in waters around the North American coast from the Pacific to the Atlantic: Implications for freshwater sources to the Arctic throughflow. Journal of Marine Research, 68:97-117.

Yeghicheyan, D., Carignan, J., Valladon, M., Bouhnik Le Coz, M., Le Cornec, F., Castrec-Rouelle, M., Robert, M., Aquilina, L., Aubrey, E., Churlaud, C., Dia, A., Deberdt, S., Dupré, B., Freydier, R., Gruau, G., Hénin, O., de Kersabiec, A.-M., Macé, J., Marin, L., Morin, N., Petitjean, P., and Serrat, E. (2001). A Compilation of Silicon and Thirty One Trace Elements 
Measured in the Natural River Water Reference Material SLRS-4 (NRC-CNRC). Geostandards Newsletter, 25(2-3):465-474.

YSI Incorporated (2009). YSI Professional Plus User Manual.

Zang, C., Huang, S., Wu, M., Du, S., Scholz, M., Gao, F., Lin, C., Guo, Y., and Dong, Y. (2011). Comparison of Relationships Between pH, Dissolved Oxygen and Chlorophyll a for Aquaculture and Non-aquaculture Waters. Water, Air and Soil Pollution, 219:157-174.

Zhou, B. and Dahlin, T. (2003). Properties and effects of measurement errors on 2D resistivity imaging surveying. Near Surface Geophysics, pages 105-117. 



\section{APPENDIX A}

\section{D DC resistivity study of the saline interface shape}

Along with the seasonal measurements made in February 2016 before the rain event (i.e. Feb1 data set) in Te Horo, six additional $155 \mathrm{~m}$ long transects (P1-P6 in Fig. A.1), centred at and perpendicular to TH1 were collected intersecting TH1 at its first $(0 \mathrm{~m}), 10 \mathrm{~m}, 20 \mathrm{~m}, 30 \mathrm{~m}, 40$ $\mathrm{m}$ and $50 \mathrm{~m}$ electrode positions. These were all measured on the same day as TH1 and using the same electrode geometry and setup as the main profile (see Section 4.2).

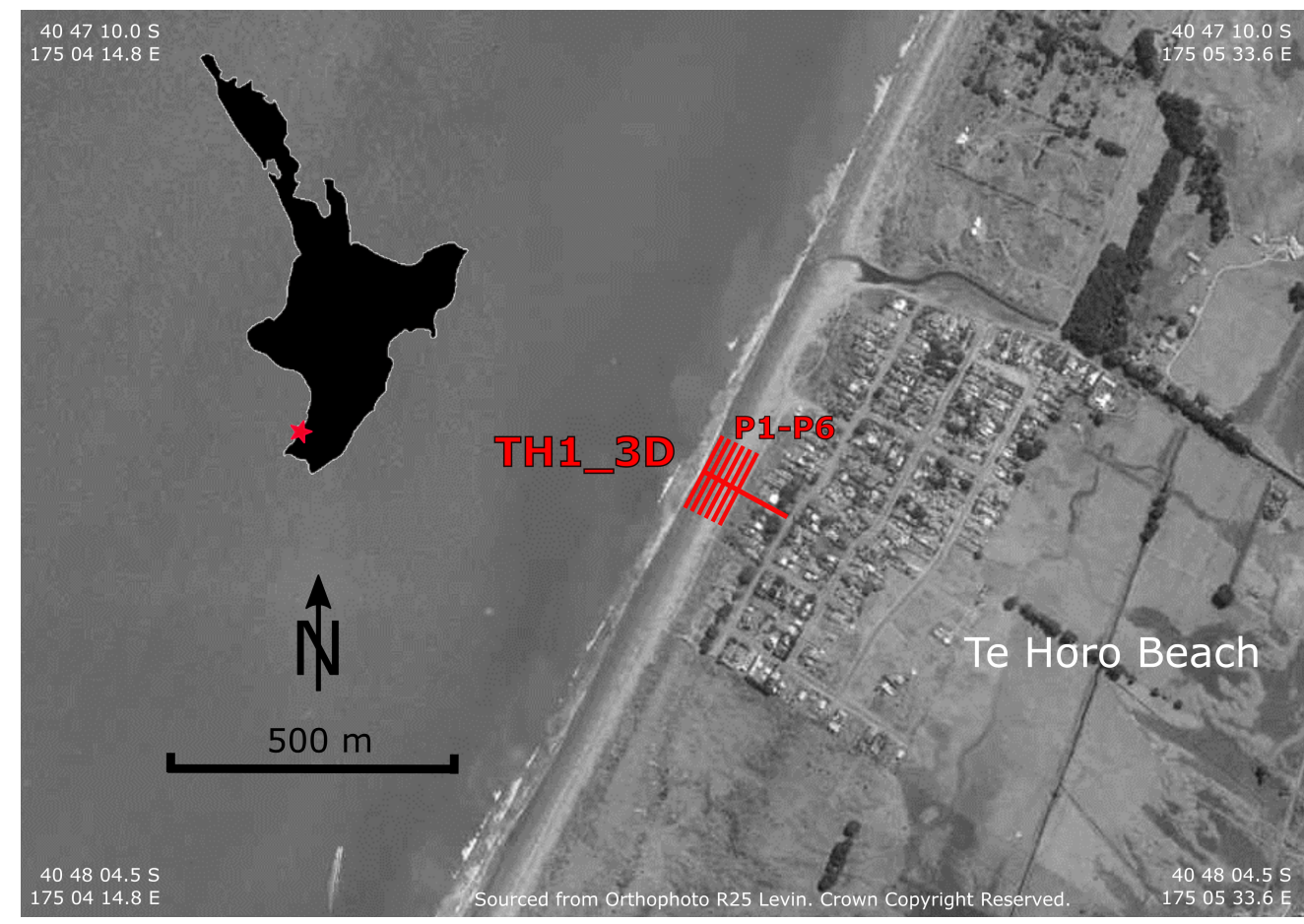

FIGURE A.1: Map of additional geoelectrical surveys in Te Horo Beach. The $\mathrm{TH}_{3 D}$ survey was done once in order to get a better understanding of the saline interface shape. P1-P6 mark six profiles perpendicular to the TH1 transect. 
The seven data sets were then combined together in order to perform a 3D inversion in Res3DInv (32-bit version) (Geotomo, Malaysia) using the same inversion parameters and strategy as was used to process the time-lapse inversions of the 2D profiles (see Section 4.3). However, with Res3DInv a DOI index analysis was not possible because the program version does not allow a background model resistivity to be incorporated. It was not attempted to use a different inversion software in order to keep consistency throughout the $\mathrm{PhD}$ project. For model appraisal the integrated model sensitivity contour lines were therefore superimposed on the resistivity model section shown in Fig. A.2. Model parts for which a sensitivity value of below 0.6 was found were considered unreliable and hence, completely cut. This approach is not optimal as the choice of the cut-off factor is subjective, and the use of the resolution matrix would be favourable, however this is not given in the 32 bit version of Res3DInv. The sensitivity information at least gives some impression of how well the model is constrained by the data.

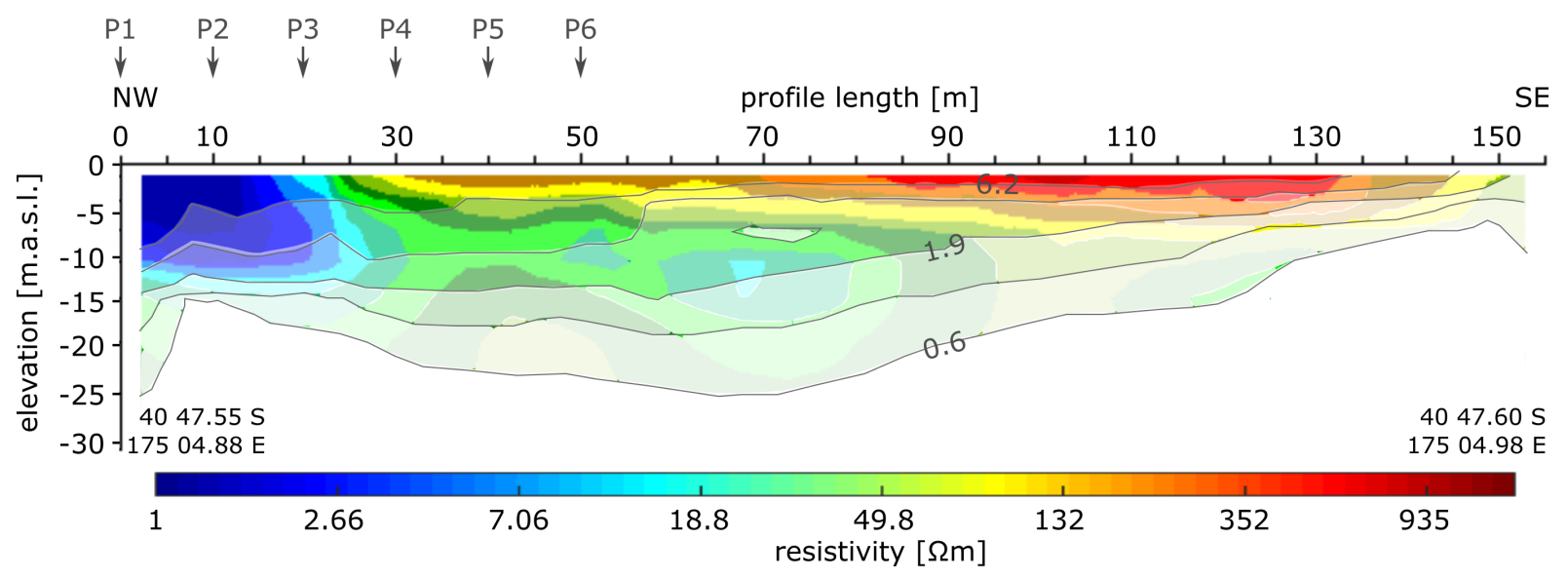

FIGURE A.2: Vertical slice along the TH1 profile line of the 3D inverted DC resistivity data from a measurement made along TH1 and six perpendicular transects (P1-P6) at the north-western end of TH1 in February 2016. Shaded contours indicate sensitivity where values below 0.6 were regarded having no model reliability.

The aim of inverting the profiles together in a 3D inversion was to investigate how the shape of the saline zone would extend further out to the sea at the north-western end of the profile, because due to the trapezoidal shape of the $2 \mathrm{D}$ transects this zone usually was not very well resolved for the Te Horo data sets compared to the Waikanae transects which were imaging a larger zone of the saline water saturated part of the aquifer. In Fig. A.2 the vertical xz-section along the TH1 profile of the 3D model is imaged. Incorporation of the six perpendicular profile lines P1 to P6 increases the sensitivity at the north-western edge of the model. This is also observed in Fig. A.3 for the horizontal depth slices through the 3D model, where the sensitivity 
is only high enough in areas covered by the profiles (i.e. the first $\sim 60 \mathrm{~m}$ on W-E extension and along TH1).

Both figures indicate a saline wedge that extends to a depth of approximately $10 \mathrm{~m}$ below mean sea level and appears to continue to an unknown extent west towards the sea. From Fig. A.3 it becomes clear that this saline wedge also is continuous in north-south direction for the entire depth section as opposed to marking a local situation around the TH1 transect. As is already clear from the profiles collected in the Waikanae groundwater zone, this does not agree with the classical Ghyben-Herzberg shaped saline interface (see Fig. 1.1) and instead suggests an unstable condition in terms of density relationships, where denser seawater overlies less dense freshwater. The three situations in which such unstable conditions appear were pointed out by Custodio (1987). They correspond to the relatively wide tidal zone at both field locations (i.e. several tens of meters), possible discharge of freshwater below the mean sea level and/or, as seen from the seasonal monitoring in Te Horo (Fig. 4.7), high disturbance from sea storms. All of these may contribute to the explanation of the observed interface situation along the Kapiti Coast. 

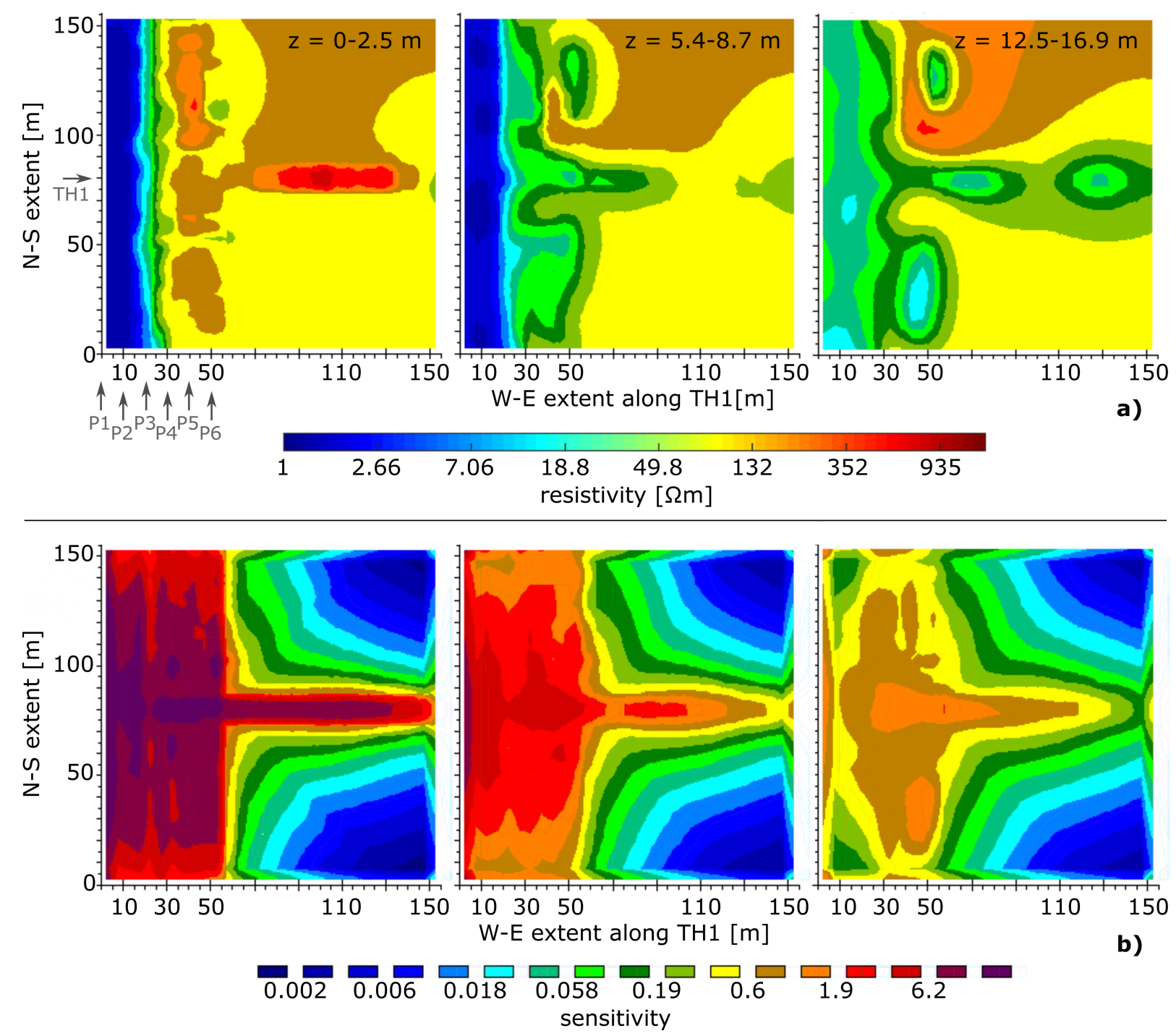

FIGURE A.3: a) Horizontal slices at three depth levels through the 3D model that was produced from the resistivity data collected along the TH1 profile and the six additional transects perpendicular to it. b) Sensitivity maps of the models at the three depth levels shown in (a). Note that areas in the models (a) with a sensitivity (b) of below 0.6 (blue to yellow) are considered unreliable. 


\section{APPENDIX B}

\section{Saline mixing front velocity}

In order to further characterise saline mixing at the field sites the Waikanae N2 and S2 profiles, as well as the Te Horo TH1 profile were used to estimate a mixing front velocity from three different resistivity isolines. Following Chapter 6, the resistivity contour line values were based on the formation factor found by Wilson et al. (2006) and electric conductivity measurements

1. the freshwater cut-off isoline of $140 \Omega \mathrm{m}$ for $0 \%$ saline mixing,

2. the $45 \Omega \mathrm{m}$ isoline representing $1 \%$ saline mixing at the field sites, and

3. the $6 \Omega \mathrm{m}$ isoline that indicates $10 \%$ mixing of seawater with freshwater.

Horizontal and vertical maximum velocity estimates for each isoline type were calculated by taking three random measurements for horizontal and vertical directions covering the maximum range of all isolines in a time series (i.e. seasonal and tidal if available). These three ranges were then averaged and divided by the time span they covered in order to end up with a resistivity change velocity value measured in $[\mathrm{cm} /$ day]. Error sources for this analysis are resulting from the DC resistivity inversion (i.e. the RMS errors) and errors from measuring the distance between the minimum and maximum contour lines $(\sim 10 \%)$. The total error for the saline mixing velocity was estimated to be the combination of the two error sources and differs between profiles depending on their resistivity model RMS errors. A table of the average estimates for several resistivity profiles in the Waikanae and Te Horo groundwater zones can be found in Table B.1. Note that the saline water mixing velocity calculated here is an average for 
the aquifer and depends on an average hydraulic conductivity of a heterogeneous mixture of geological materials with varying high and low $K$. Hydraulic conductivity is scale dependent such that for the same material covering an area of $1 \mathrm{~m}^{3} \mathrm{~K}$ will be different than for an area covering $1 \mathrm{~km}^{3}$ (Rovey and Cherkauer, 1995). The saline water mixing velocity (or in general water flow velocity) as presented in Table B.1 should not be mistaken as being equivalent to hydraulic conductivity, however. Groundwater flow velocity $(v)$ is calculated from $K$ according to Darcy's law by

$$
v=\frac{K^{d h} / d l}{n}
$$

where $d h / d l$ is the hydraulic gradient and $n$ the porosity. Gyopari et al. (2014) estimated the hydraulic gradient in the shallow aquifer at the Kapiti Coast to be in the order of 0.001. Porosity can be assumed to be in the order of 0.25 for a gravelly sand (Johnson, 1967). Using these values in Eq. B.1 for an estimated hydraulic conductivity of $56 \mathrm{~m} /$ day for the coarse sandy gravel layer identified on the DC resistivity transects at the field sites results in a freshwater flow velocity of roughly $22 \mathrm{~cm} /$ day for this layer (also called feature 3 in Chapter 4). This is very close to the groundwater velocity that can be calculated for the Q2 aquifer ( $K$ of 10-50 $\mathrm{m} /$ day, porosity of about $25 \%$ ) at 4-20 cm /day, which makes sense considering that the Q2 aquifer is made up of gravelly sand. In contrast, taking the estimated hydraulic conductivity of about $5 \mathrm{~m} /$ day (Gyopari et al., 2014) for the Holocene sand aquifer (porosity of around 0.35 (Johnson, 1967)) at the Kapiti Coast, which is possibly a representative value for the bulk aquifer lithology, a groundwater flow velocity of $1.5 \mathrm{~cm} /$ day is calculated.

Two general things can be noted directly from Table B.1. First, from velocity ratios it appears as if for more than $75 \%$ of the cases the horizontal velocity is larger than the vertical velocity. The other common observation is that tidal saline front movements are roughly one to two magnitudes faster than those estimated from seasonal time series. This is possibly a consequence of the higher resolution of the tidal profiles (2-m minimum spacing vs. 5-m minimum spacing for seasonal time series), which allows observation of the faster changes induced by the tidal movements in the wave run up and near-shore zones that are not resolved in the seasonal monitoring data sets. This is a further indication that the tidal water movement is not resolved in the seasonal monitoring data sets. Another factor may be that in the more highly resolved tidal monitoring data larger portions of the hydraulically more conductive 
TABLE B.1: Average saline mixing front velocities derived from three different isolines and their temporal expansion. The column value refers to the isoline resistivity plus indication of horizontal or vertical velocity estimate or ratio of these two velocity estimates. The error estimate for the velocity of each profile is given on the line following the profile name in percentage and underneath each measurement in absolute values.

\begin{tabular}{l|ccc|ccc|ccc}
\hline \multirow{2}{*}{ Profile } & \multicolumn{3}{|c|}{$v_{\text {horizontal }}[\mathrm{cm} /$ day] } & \multicolumn{3}{c|}{$v_{\text {vertical }}[\mathrm{cm} /$ day] } & \multicolumn{3}{c}{ ratio } \\
& 140 & 45 & 6 & 140 & 45 & 6 & 140 & 45 & 6 \\
\hline N2 season & 1.38 & 1.15 & 1.71 & 0.91 & 0.76 & 0.61 & 1.52 & 1.52 & 2.81 \\
$\pm 12.5 \%$ & 0.17 & 0.14 & 0.21 & 0.11 & 0.1 & 0.08 & 0.27 & 0.27 & 0.5 \\
N2 tidal & 118.9 & 121.34 & 149.0 & 89.36 & 122.0 & 106.51 & 1.33 & 0.99 & 1.4 \\
$\pm 15.1 \%$ & 17.95 & 18.32 & 22.5 & 13.49 & 18.42 & 16.08 & 0.28 & 0.21 & 0.3 \\
S2 season & 1.05 & 0.86 & 1.27 & 0.93 & 0.86 & 0.67 & 1.13 & 1.0 & 1.89 \\
$\pm 12.5 \%$ & 0.13 & 0.11 & 0.16 & 0.12 & 0.11 & 0.08 & 0.2 & 0.18 & 0.33 \\
TH1 season & 4.51 & 3.59 & 3.82 & 1.76 & 1.2 & - & 2.57 & 2.98 & - \\
$\pm 12.7 \%$ & 0.57 & 0.46 & 0.49 & 0.22 & 0.15 & - & 0.46 & 0.54 & - \\
TH1 tidal & 84.99 & 243.19 & 365.34 & 99.47 & 215.83 & 111.37 & 0.85 & 1.13 & 3.28 \\
$\pm 11.8 \%$ & 10.03 & 28.7 & 43.11 & 11.74 & 25.47 & 13.14 & 0.14 & 0.19 & 0.55 \\
\hline
\end{tabular}

layer with estimated $22 \mathrm{~cm} /$ day groundwater flow velocity, is imaged compared to the spatially larger seasonal time series containing more of the $1.5 \mathrm{~cm} /$ day groundwater movement rate.

There is consistency in the saline mixing front (i.e. resistivity) velocities between the three different isolines observed. However, there seems to be a significantly (2-3 times) higher water movement in the Te Horo Beach aquifer for both seasonal and tidal estimates compared to the Waikanae locations, although this is not as pronounced for the freshwater isoline estimates of the tidal velocity. A faster fluid mixing velocity in Te Horo as opposed to Waikanae ties in with the high hydraulic conductivity (56 m/day) estimated from tidal head attenuation calculations in Chapter 6 for the coarse sand and gravel layer possibly associated with the gravel ridges in Te Horo Beach. However, from the time-lapse models of the Waikanae profiles there is likely a higher hydraulic conductivity found in the middle layer as outlined in Sections 4.4.1 and 5.3.1, but is possibly not quite as high as in Te Horo.

There is no significant saline mixing velocity difference between the rural N2 and urban S2 profiles in the Waikanae groundwater zone. Hence, aquifer locations in more urban and rural areas does not appear to be a dominating factor for seawater intrusion velocity in the Waikanae groundwater zone when compared to the hydrogeological settings. This means that it is much more likely that differences in aquifer lithology, and therefore variations in their hydraulic parameters, of the Waikanae and Te Horo field areas are leading to the observed differences 
in saline mixing front velocity estimates, DTW time lags and effectively seawater intrusion potential, rather than Te Horo's urban TH1 location as compared to the Waikanae N2 profile. However, urbanisation and its change to natural recharge and discharge processes may still be an important local factor in controlling seawater intrusion into a coastal aquifer as was indicated by the unexpected temporal behaviour of the geoelectrical and hydrochemical measurements.

In a comparable hydraulic environment (hydraulic conductivity $K \sim 10^{-3} \mathrm{~cm} / \mathrm{s}$ versus the Q1 Holocene sand aquifer Kapiti Coast $K=2.3^{*} 10^{-3}-5.8 * 10^{-3} \mathrm{~cm} / \mathrm{s}$ (Table 2.1)). De Franco et al. (2009) estimated seasonal saline mixing front velocities for an aquifer under investigation in northern Italy, that were in the order of 1-10 cm/day, and hence are in a similar range as the ones estimated from seasonal monitoring in this study. This again demonstrates good agreement and therefore applicability of results found from one specific location to other, similar environments. 


\section{APPENDIX C}

\section{Seasonal implications from repeated tidal resistivity}

monitoring

\section{C.1 Introduction to the Te Horo tidal monitoring data sets}

As seen in Chapter 5, diurnal geoelectrical tidal monitoring data can allow inference of quite valuable hydrological information in addition to the usual imaging purpose of an aquifer. In order to investigate how the findings of the long-term study in the Waikanae groundwater zone apply to the Te Horo groundwater zone and how these change within a seasonal time frame, six shorter time series have been collected during a one-year period along the TH1 transect (Fig. 4.2). For a general introduction to the problem, techniques used and a discussion of data analysis procedures, the reader is referred to Chapter 5 . The error estimate was in the order of $1.62 \%$ to $2.49 \%$ and the statistical cut-off factor determination developed by Deceuster et al. (2014) could not be used due to unimodal distribution of DOI index values. Therefore, and a fixed cut-off factor of 0.2 as suggested by Marescot et al. (2003) and Hilbich et al. (2009) was used to scale the inversion models instead.

Tidal monitoring data sets in Te Horo Beach were acquired every three months over monitoring times ranging between 9 and 13 hours. The groundwater level and tidal range data for each of the six DC resistivity acquisition days are given in Table C.1, and the relative position of each monitoring with respect to the seasonal hydrological cycle is illustrated in Fig. C.1 in terms of groundwater stage and rainfall data at the Te Hapua wetland monitoring site. The 2D 
TABLE C.1: Tidal range at Otaki river mouth and groundwater level at Te Hapua wetland for the tidal monitoring acquisition dates along the Te Horo Beach TH1 profile.

\begin{tabular}{lll}
\hline Acquisition date & $\begin{array}{l}\text { Groundwater level } \\
{[\mathrm{mm}]}\end{array}$ & Tidal range (HT-LT) [m] \\
\hline 12. Nov 2015 (9:30-18:30) & 3017 & 1.82 \\
22. Feb 2016 (8:05-20:05) & 2918 & 1.77 \\
22. Apr 2016 (8:30-16:30) & 2753 & 1.82 \\
08. Jun 2016 (9:00-17:00) & 3065 & 1.78 \\
12. Sep 2016 (9:30-17:30) & 3145 & 0.73 \\
19. Sep 2016 (8:20-16:20) & 3464 & 2.33 \\
\hline
\end{tabular}

time-lapse resistivity data sets described herein were collected over varying monitoring periods depending on available daylight (i.e. time series in summer are generally longer). While the tidal range was similar (average of $1.8 \mathrm{~m}$ ) for the first four time-lapse measurements (November 2015 - June 2016), those acquired in September 2016 were carried out first during a low tidal range $(0.73 \mathrm{~m})$ and only a week later during a high tidal range $(2.33 \mathrm{~m})$ in order to investigate the importance of the tidal magnitude on seawater intrusion.

November 2015 and June 2016 data sets were collected in similar hydrologic conditions, however, only two weeks prior to the June data acquisition a strong sea storm had washed over the Kapiti Coast, which shifted the drift wood and surface gravels by about $40 \mathrm{~m}$ further inland (east) and flooded the beach face with seawater up to approximately $80 \mathrm{~m}$ profile extent on TH1 (i.e. the entire model section in the tidal monitoring data sets). This was followed by a similar event in late July 2016, introducing more seawater seepage from the surface. February and April 2016 data sets were acquired during the driest time of the year and may therefore also be comparable. However, there was significant rainfall just before the February data collection. This was also a problem with the September 2016 data sets that were initially planned to be collected in similar hydrologic conditions but differing tidal stages. However, after acquiring the low tidal stage data (Sep1), reasonably heavy rainfall (up to $7.5 \mathrm{~mm} /$ hour) occurred just before collecting the second, high tidal stage data set (Sep2). This shows how difficult such campaigns can be in terms of planning, and also suggests that installed, automated systems would be helpful because they can be operated in a more flexible way. However, there are cost and permit issues tied to such systems, which currently prevents them from being used more extensively. With the available data sets presented in the following, comparison of small scale changes like the tidal influence on resistivity time series may be difficult due to such unforeseeable events. Some valuable information can be extracted nevertheless. 

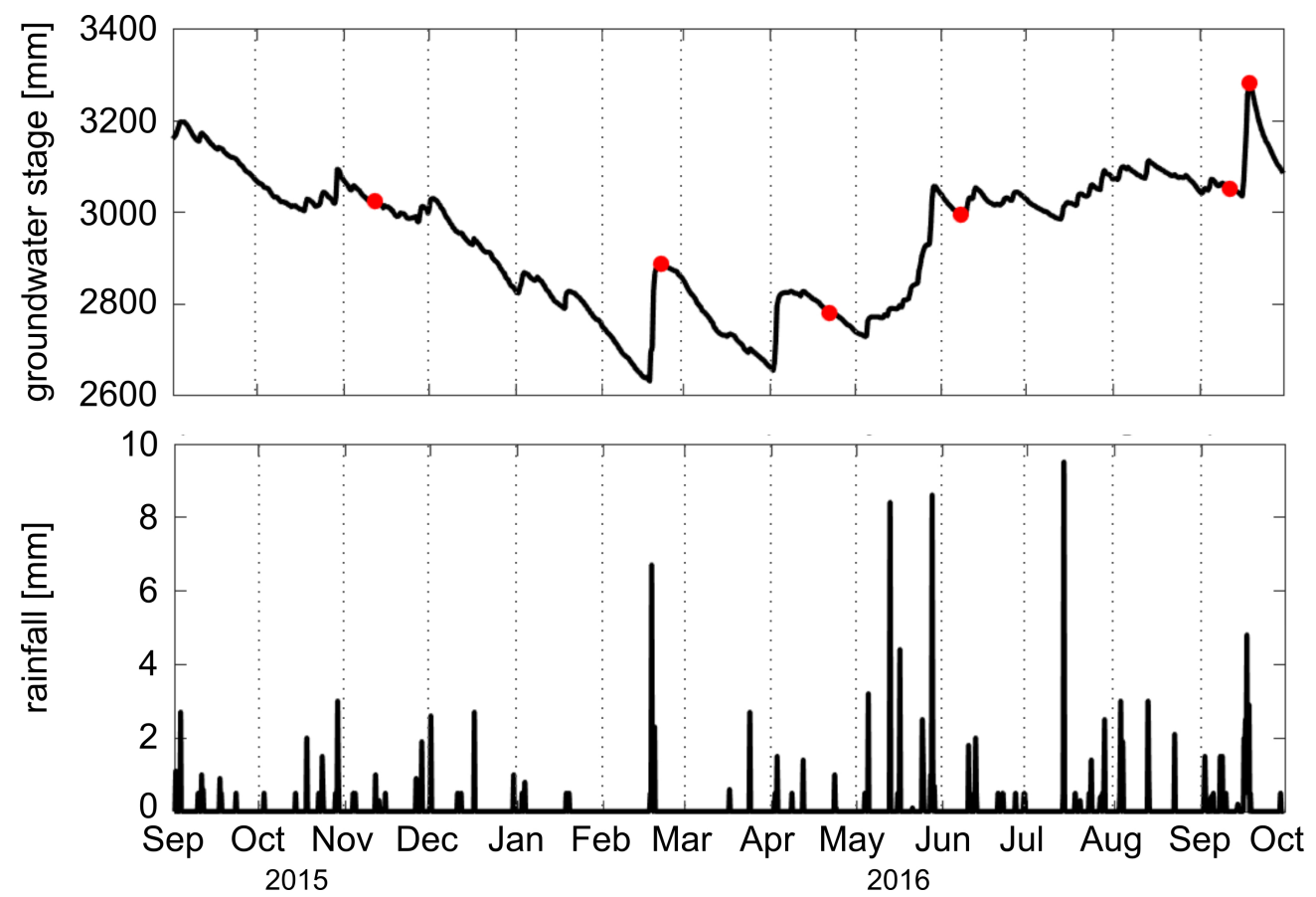

FIGURE C.1: Groundwater stage (top) and rainfall (bottom) measured at the Te Hapua wetland GWRC environmental monitoring network station (location see Fig. 2.1) from September 2015 until October 2016. Red dots indicate the times of the DC resistivity tidal monitoring dates along profile $\mathrm{TH} 1$.

\section{C.2 The tide's seasonality - implications from resistivity ra- tios and time series}

In Fig. C.2 the resistivity ratios between high tide and low tide (LT/HT) are shown for all six tidal monitoring data sets. The ratios between consecutive time steps in each data set were typically below $5 \%$ (see Appendix D) and therefore so small that it would be hard to make any statements based on these, which is why only ratios between the tidal extremes are discussed here. And even from these, it is relatively hard to identify areas of the subsurface showing different behaviour because the absolute resistivity changes during the monitoring periods appear to be fairly small $(<5 \Omega \mathrm{m})$ for large parts of the profile space in monitoring data sets between April and September 2016. These low resistivity changes between time steps may point towards the implication of tidal forcing not having a major influence on the resistivity changes at this location. Parts that increase in resistivity with decreasing tidal stage are again marked with $A$, whereas those with a decreasing resistivity going from high to low tide are highlighted with the letter $B$. Although, the first three and the last three profiles show similar outlines in 
their resistivity ratios within their respective groups, there seems to be a relative change of the behavioural pattern with time in terms of which areas react in which way. These shifts of areas identified as $A$ and $B$ may be a direct consequence of how the signals are related to each other within the differing monitoring windows for different acquisition dates as was discussed for the synthetic example in Appendix E. In addition, the amplitude of the resistivity ratios appear to be different at different times during the year and is more pronounced (20-60\%) for the November and February data sets but is considerably lower $(<20 \%)$ in April and June for comparable tidal ranges. A similar observation can be made when the resistivity ratios of the two September monitorings are compared. At a tidal range of less than one meter the resistivity ratio between high and low tide is basically non-existent, which indicates that there is very little (resolvable) change in resistivity within a tidal cycle at a small tidal range. This stands in contrast to the modelling results of Ataie-Ashtiani et al. (1999) who found significant changes for small tidal ranges, but agrees with the conclusions made by Morrow et al. (2010) who found no significant influence on the resistivity signals during low tidal ranges in the Waikanae groundwater zone. Considering the use of a different method and dealing with a heterogenous ground in the field study discussed here, the discrepancy to Ataie-Ashtiani et al. (1999) is not entirely surprising, however. If the tidal range is larger (i.e. $2.33 \mathrm{~m}$ only one week later) then this markedly changes the amplitude of the resistivity ratio between tidal extremes (up to $40 \%$ change).

The tidal monitoring data sets in November 2015 and June 2016 were measured at times when the groundwater stage was similar. The November measurements came at a time of the year when rainfall has started to reduce, while the measurements in June 2016 followed a series of significant rain events leading into winter (Fig. C.1). As the tidal range for these data sets was similar then the most likely parameter affecting the amplitude of resistivity change between high and low tide is the degree of freshwater flow through and into the shallow part of the aquifer. This could be expected to be greater in June 2016 following the rain events and, consequently, it might be expected that this would diminish the degree to which the resistivity varies between high and low tide. This was observed in the model study by Ataie-Ashtiani et al. (1999), and is indeed seen in Fig. C. 2 where the resistivity ratios for the June 2016 data are much closer to unity than are those for the November 2015 data for which more significant changes are observed. However, the flooding of the beach face two weeks prior to the June measurements 


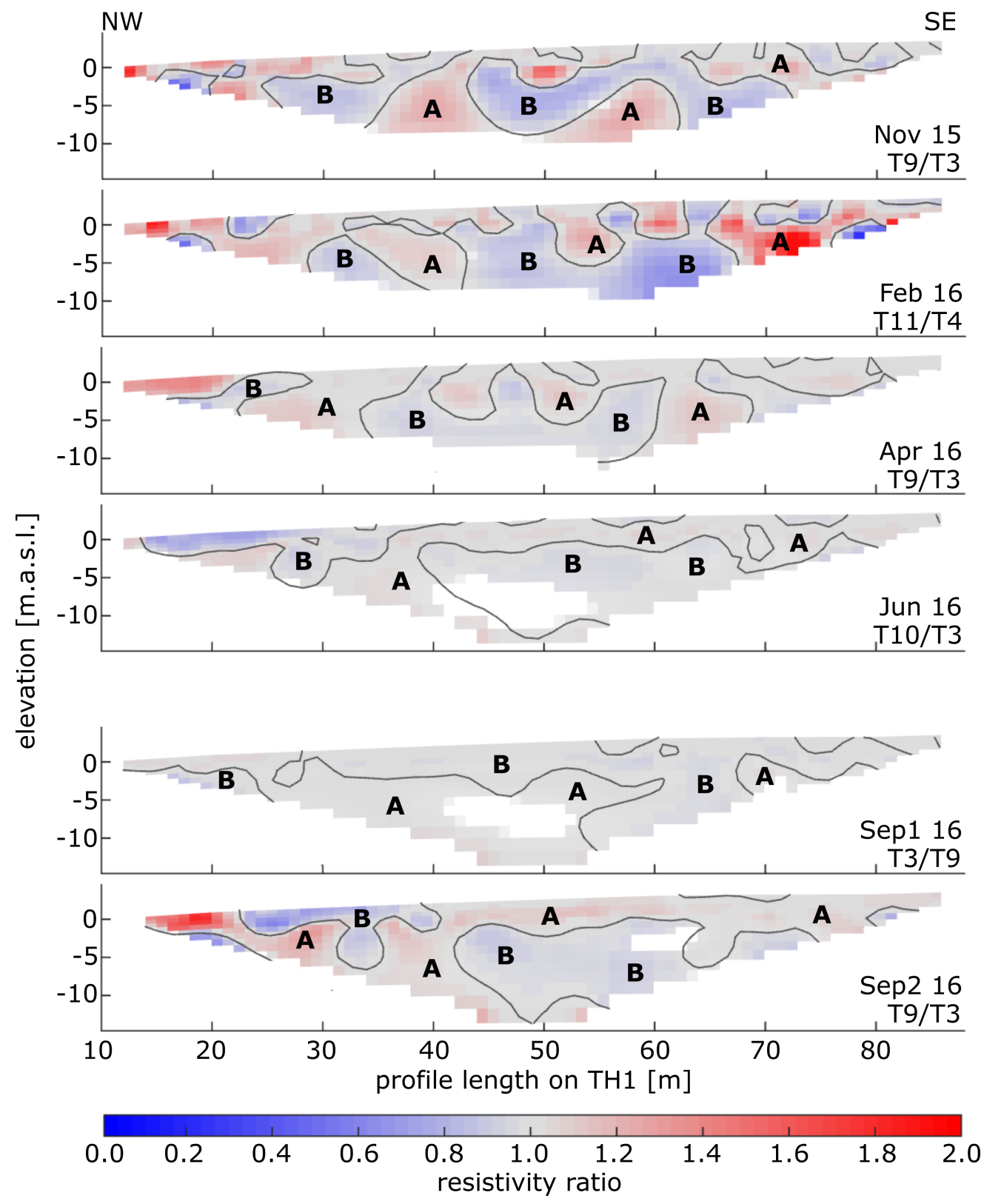

FIGURE C.2: Resistivity ratios between high and low tide (or between time steps 6 hours apart) for all of the six tidal monitorings carried out along the Te Horo Beach TH1 profile (see Fig. 4.2). Letters $A$ refer again to parts of the aquifer that react in the expected way with decreasing tide, whereas letters $B$ mark those areas that behave in the opposite way. 
may mean that the subsequent downward filtration of seawater from this event also contributes to the smaller resistivity changes.

There were also similar tidal ranges for the measurements in February and April 2016 at times when the groundwater stage was somewhat lower but had recently increased following rainfall events (Fig. C.1). In both these two cases, although the percentage resistivity changes for the February data are somewhat larger, the amplitude of actual resistivity changes are more significant than for April 2016. The slightly more significant changes for the February measurements compared to those in April may reflect the fact that these were made immediately following a rain event with little time for increased freshwater flow to be manifest.

Although the second September 2016 measurements followed a significant increase in groundwater stage, and presumably increased freshwater flow, the amplitude of resistivity changes are much larger for the $2.33 \mathrm{~m}$ tidal range associated with this data set than for the earlier September 2016 data set, prior to the rise in groundwater level, for which there was only a $0.73 \mathrm{~m}$ tidal range. Again, it is likely that the significant rain event prior to the second set of measurements was not yet reflected in freshwater flow, and instead the difference reflects the importance of the tidal range in the amount of seawater mixing that occurs. Similar to what Morrow et al. (2010) concluded in their study across a beach section in Waikanae (north of profile S3 discussed in Chapter 4), and Turner and Acworth (2004) had pointed out for changes in the saline interface, the two September monitoring data sets strongly suggest that a large tidal range leads to significantly more saline mixing in a coastal aquifer than is the case for small changes in tidal stage between low and high tide.

\section{C.3 Summary}

Similar to the Waikanae tidal monitoring presented in Chapter 5, model parts with an increase in resistivity during a rising tide were identified with letter $A$, while those indicating a decrease with increasing tidal stage were termed $B$. Results from the Te Horo TH1 profile generally show smaller time lags for both $A$ and $B$ regions than was observed for the Waikanae study. This may be a result of differing hydraulic conductivity between the two field sites due to their locations 
in more or less sandy and gravelly environs, respectively. Freshwater flow is indicated to be a controlling factor for seawater intrusion at the study site with more freshwater available due to higher recharge from continuous rainfall leading to a significantly lowered amplitude in resistivity change during a tidal cycle. A further complicating factor in temporal relationships between tidal and resistivity signals is likely accounted to highly varying hydraulic conductivity within the shallow coastal aquifer (i.e. hydrogeologic heterogeneity as indicated from bore logs as well as hydrochemical, and groundwater EC fluctuation measurements discussed in Chapter 6) that leads to complex time lag relationships.

Due to their short length, tidal monitoring series along profile TH1 could not be used for determining resistivity periods from autocovariance. However, as presented in Appendix F, using the periods obtained for groundwater stage fluctuations in a closely situated well in Te Horo Beach indicates a longer period for resistivity signals of about 21 hours at this location as well. At the same time this study again confirms the possibility of having longer periods for resistivity signals as compared to the tidal signal as was found from autocovariance analysis of the Waikanae time series in Section 5.3.2. In general, the time series discussed in this appendix appear to be rather short for a complete description of the hydraulic characteristics. It once more points out the necessity of having time series spanning at least two full cycles of the signal.

For a sustainable management of groundwater resources in coastal areas it is a necessity to know the factors that influence the aquifer structure and hydrology. Preferential flow paths are an important feature and are vital to be understood and identified in terms of these providing a direct pathway for seawater to intrude deeper and to extended parts of a freshwater aquifer. Especially with regard to rising sea levels and overexploitation of aquifers in coastal areas around the world. The tidal monitoring studies presented in Chapter 5 and this appendix chapter clearly demonstrated the possibility of extracting information to assess qualitatively the hydraulic properties from DC resistivity monitoring data sets. However, it is advisable to obtain longer time series for a more robust assessment. 

APPENDIX D

TH1 tidal monitoring resistivity ratios 


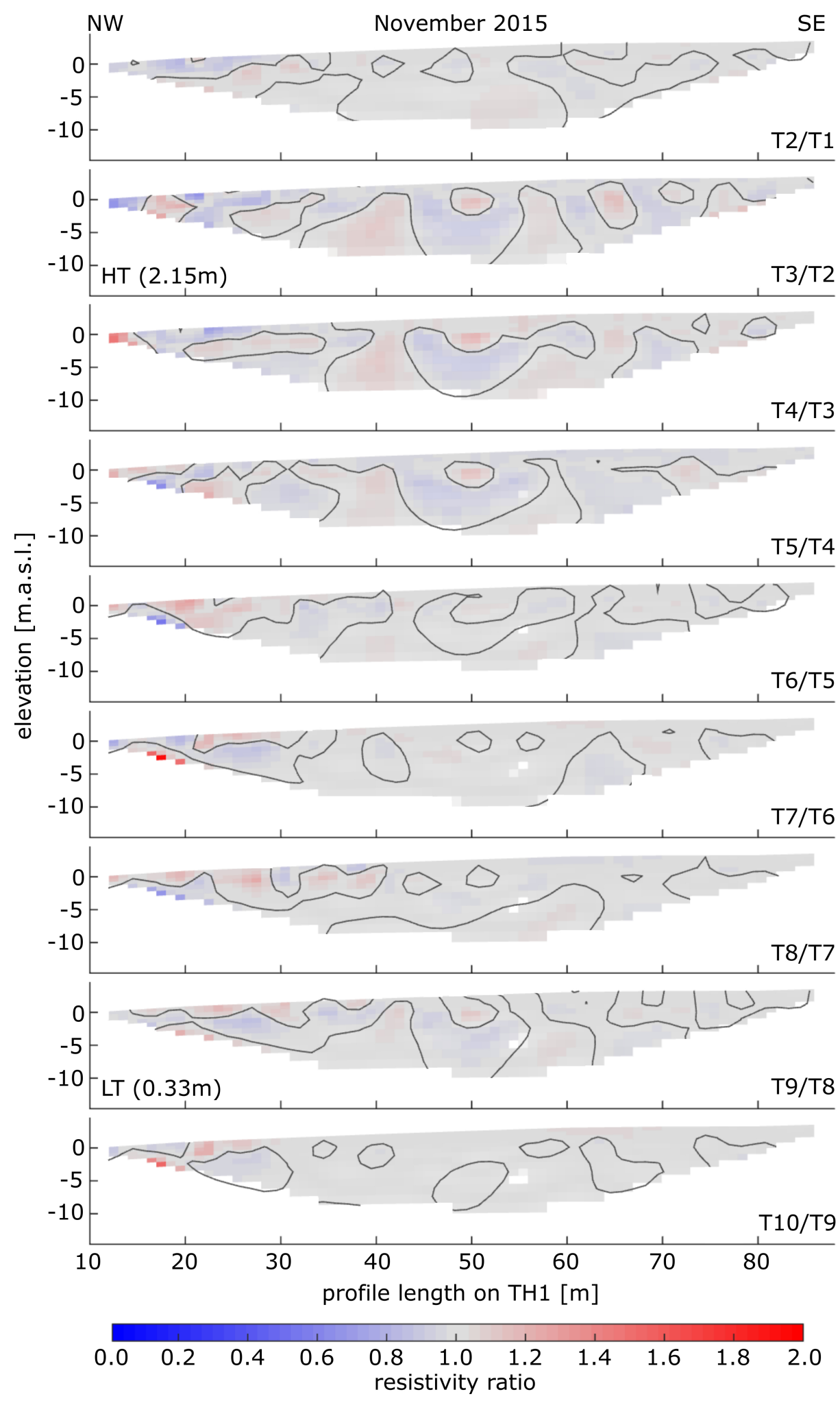

FIGURE D.1: Resistivity ratios between each consecutive time step for the November 2015 tidal monitoring along the TH1 profile 


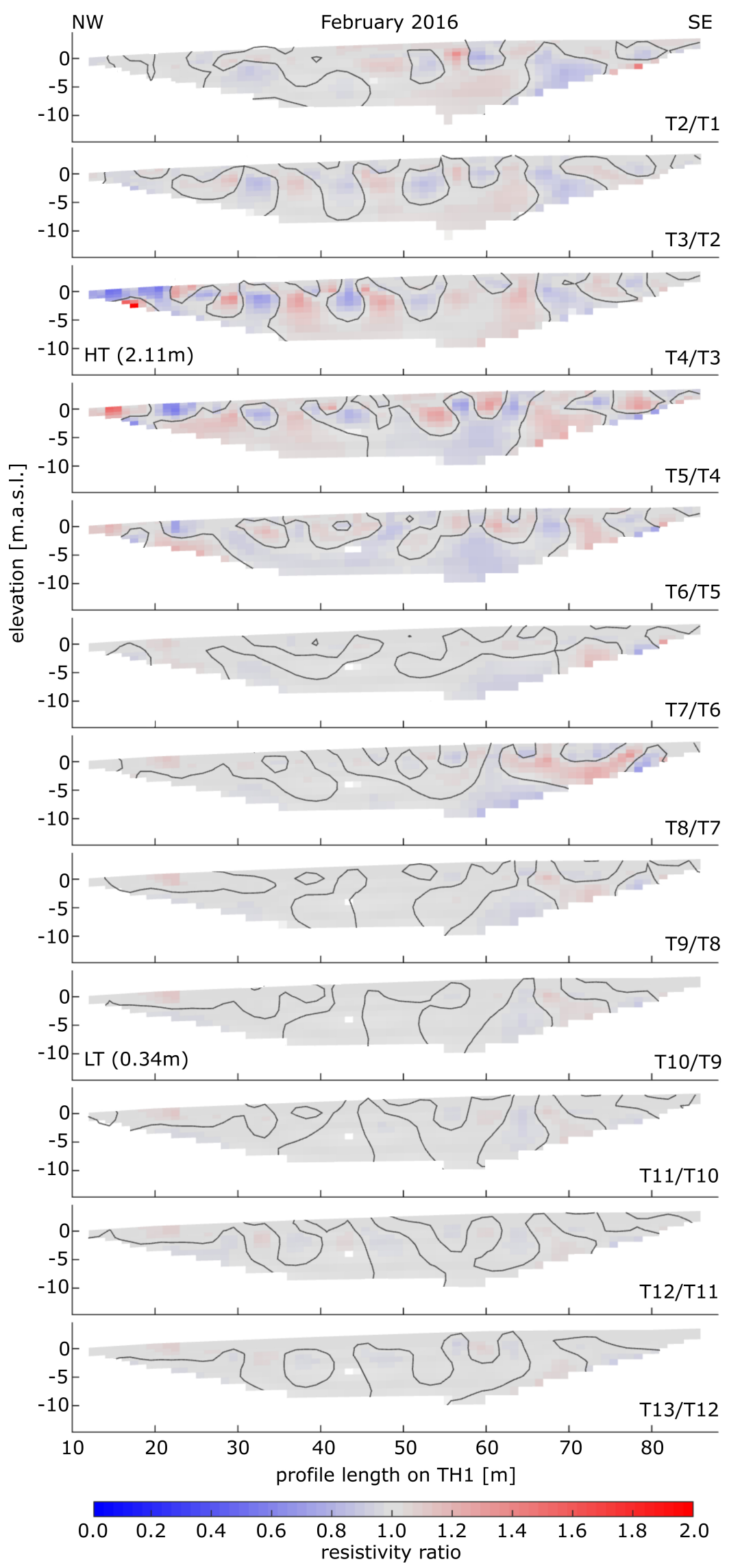

FIGURE D.2: Resistivity ratios between each consecutive time step for the February 2016 tidal monitoring along the $\mathrm{TH} 1$ profile 


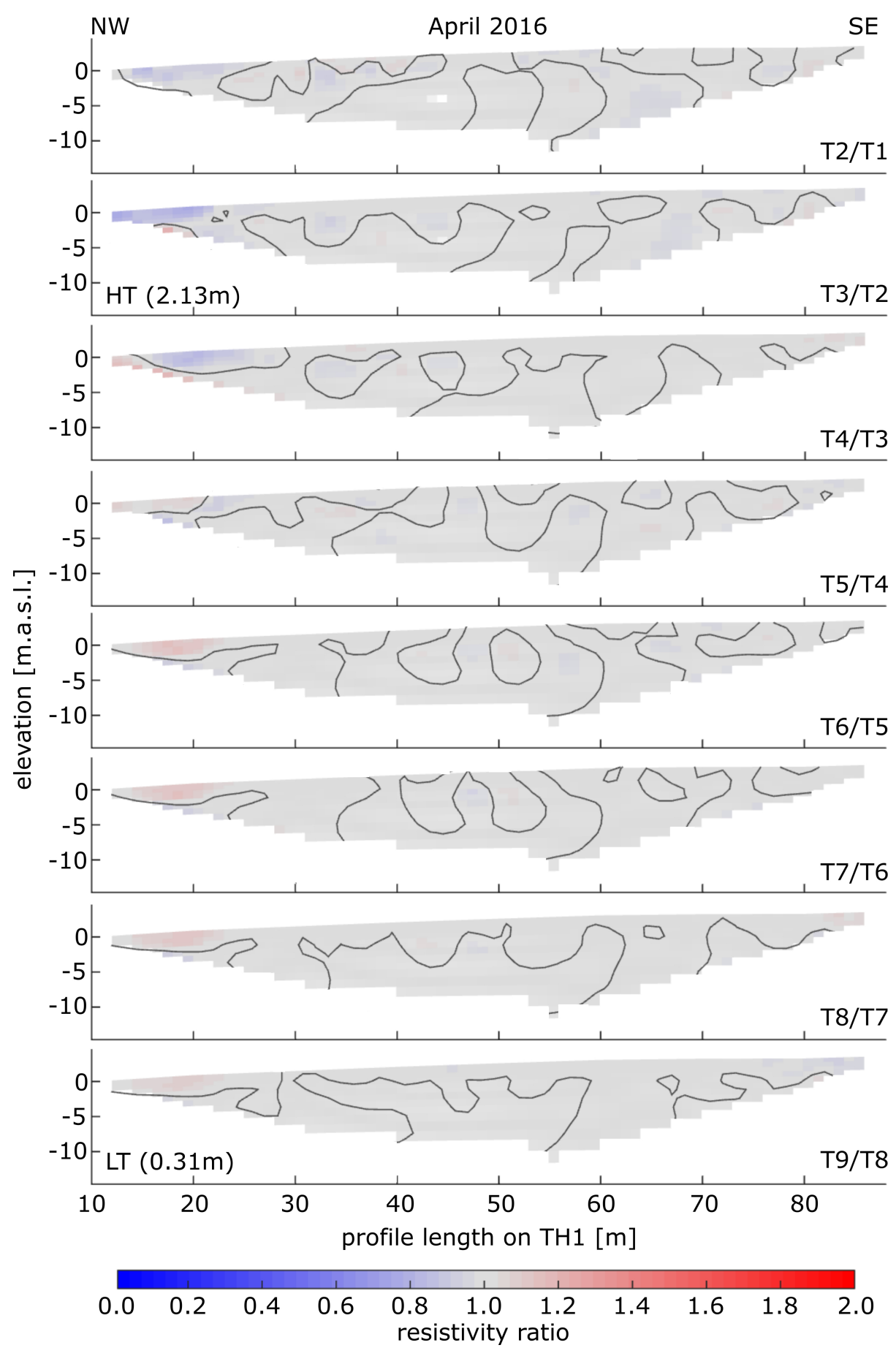

FIGURE D.3: Resistivity ratios between each consecutive time step for the April 2016 tidal monitoring along the $\mathrm{TH} 1$ profile 


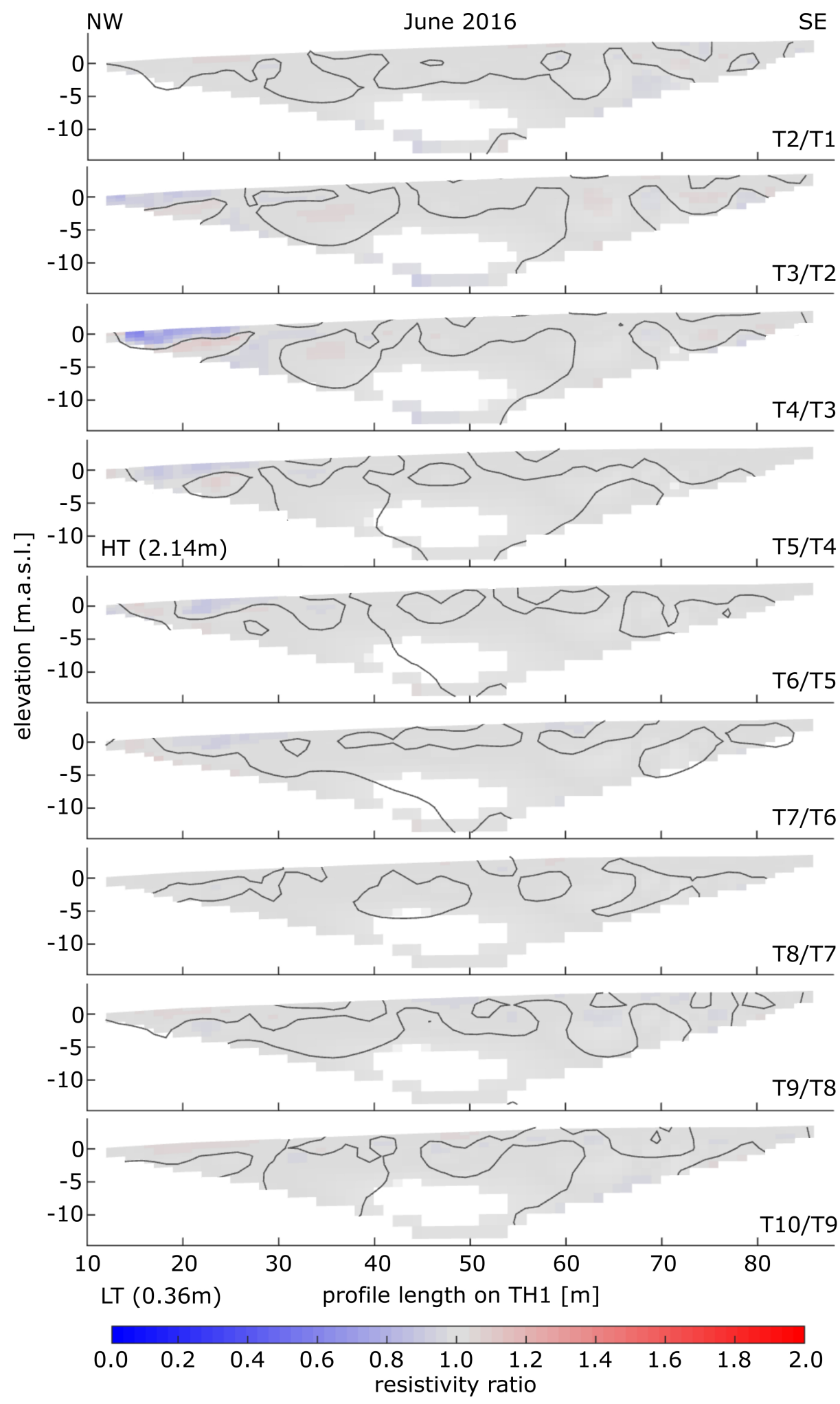

FIGURE D.4: Resistivity ratios between each consecutive time step for the June 2016 tidal monitoring along the TH1 profile 


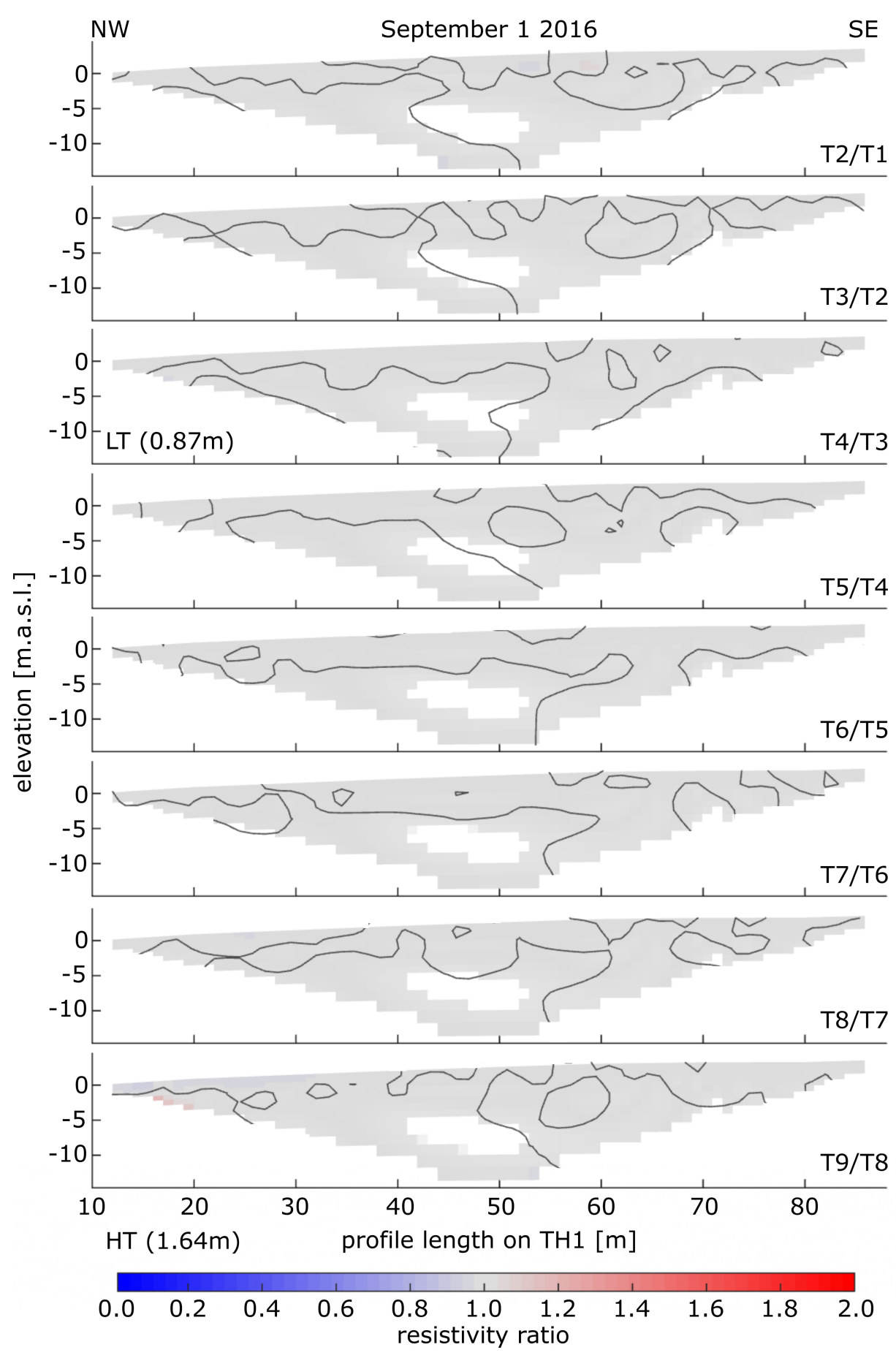

FIGURE D.5: Resistivity ratios between each consecutive time step for the first (small tidal range) September 2016 tidal monitoring along the TH1 profile 


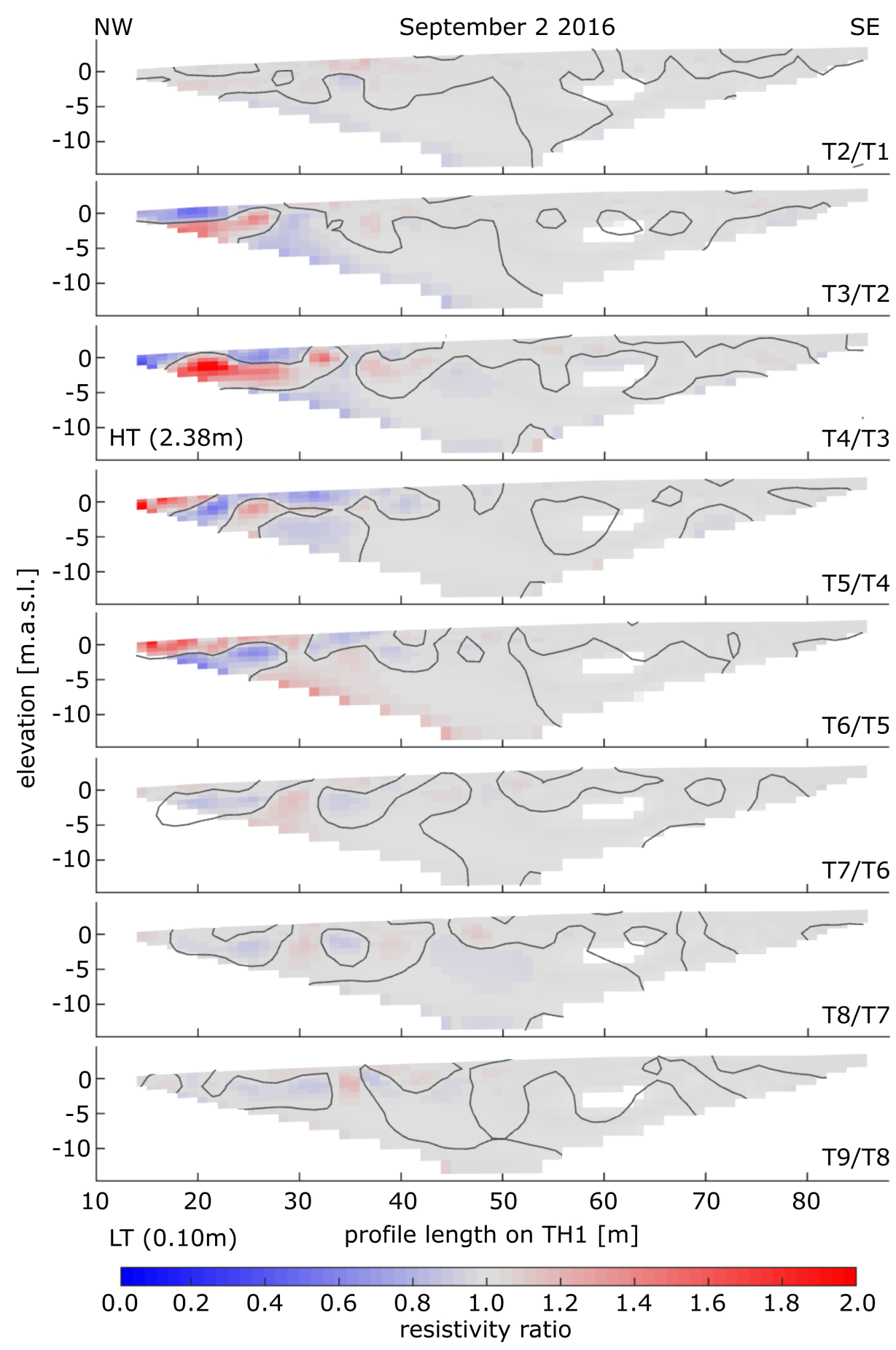

FIGURE D.6: Resistivity ratios between each consecutive time step for the first (large tidal range) September 2016 tidal monitoring along the TH1 profile 



\section{APPENDIX E}

\section{Tidal DTW modelling for a better understanding of field data}

The discussion of the results of the 21-hour monitoring in Waikanae in Chapter 5 illustrate the problems associated with analysing a field data set for recurring resistivity patterns during a tidal cycle and identifying hydraulic parameters and flow paths from it. One of the concerns is that the length of the monitoring period appears to be relatively short compared to the indicated period of the resistivity signal. In order to better understand the relationships between tidal stage and resistivity signals some additional theoretical modelling was performed and is discussed in this appendix chapter. A simpler situation was simulated with signal periods of 12 hours for all three time series. One 'resistivity' signal was shifted by 2 hours and the other by 8 hours relative to the 'tidal' signal. This leads to the same temporal relationship for the two resistivity signals as observed from the field data (i.e. always out of phase), but gives better control in terms of temporal consistency of the relationship between tidal and resistivity signals. The DTW optimal warping costs (OWC) were then calculated for differing monitoring windows shifting the respective fixed window across a five-day signal in one-hour steps. In Table E.1 the standard deviations for all OWC values found for one window length across the entire five-day signal are summarised along with the mean OWC.

The OWC found through DTW for varying monitoring window lengths in Table E.1 for modelled signals $A$ and $B$ relative to a tidal stage signal have a clear trend with a smaller standard deviation for signals $A$ compared to signals $B$ independent of the actual time window. However, window lengths that are multiples of half the signal period (i.e. 12, 18, 24, 30 hours) appear to have the same characteristics. Hence, the actual window length seems to be of minor importance, whereas the length of the monitoring window relative to the signal periods appears to be more 
TABLE E.1: Optimal warping cost differences for different monitoring window lengths of two modelled resistivity signals with a period of 12 hours and actual time shifts of 2 and 8 hours with respect to a tidal signal with at 12 hour period. Standard deviations and mean values were calculated from DTW cost differences of each window shifted through a five-day signal length in one-hour steps.

\begin{tabular}{lllll}
\hline $\begin{array}{l}\text { Window } \\
\text { length }[\mathrm{h}]\end{array}$ & $\begin{array}{l}\text { Standard } \\
\text { deviation OWC } A \\
{[-]}\end{array}$ & $\begin{array}{l}\text { Standard } \\
\text { deviation OWC } B \\
{[-]}\end{array}$ & Mean OWC A [-] & Mean OWC B [-] \\
\hline 6 & 0.71 & 0.83 & 1.91 & 5.55 \\
8 & 0.87 & 2.49 & 1.89 & 5.85 \\
12 & 0.71 & 1.12 & 1.91 & 5.8 \\
15 & 0.59 & 2.25 & 1.89 & 5.86 \\
18 & 0.71 & 1.12 & 1.91 & 5.8 \\
21 & 0.59 & 2.25 & 1.89 & 5.86 \\
24 & 0.71 & 1.12 & 1.91 & 5.8 \\
30 & 0.71 & 1.12 & 1.91 & 5.8 \\
40 & 0.24 & 1.4 & 1.91 & 5.84 \\
50 & 0.87 & 2.49 & 1.88 & 5.89 \\
\hline
\end{tabular}

important. Also, a longer monitoring period does not seem to improve the OWC estimates (i.e. comparing window lengths of 12 and 30 hours or 50 hours for example). Therefore, it can be concluded that for analyses using the DTW method to estimate OWC between time series as discussed in Chapter 5, it is beneficial to choose the monitoring time according to signal periods. For a field survey this would mean to obtain a sufficiently long resistivity baseline data set from which such periods can be estimated before the actual monitoring survey.

In the case of the real field example, however, the periods between tidal and resistivity signals are clearly different from each other, and the above conclusions may only be true if signals being compared have the same period. In order to investigate a more realistic scenario two typical part $A$ and $B$ resistivity time series from the 21-hour Waikanae monitoring data set were plotted against the tidal signal at the time of the monitoring in Fig. E.1(a). From the DTW analysis shown in Fig. 5.9 OWC for conductivity time series of parts $A$ give values between 6 to 10 hours, whereas those for parts $B$ are in the order of 12 to 16 hours. Autocovariance of each model cells time series revealed resistivity periods of 18 to 20 hours for most of the analysed aquifer section in Fig. 5.11. If the three signals are graphically approximated with clean sine signals as shown in Fig. E.1(b), then for these two model cell time series $A$ and $B$ a period of about 20 hours can be estimated from the plot. This was then used to generate two sine curves simulating the bulk electrical conductivity of the aquifer in Fig. E.1(c), where they are shown 
together with the tidal stage signal (period of about 12 hours) for a 20-hour window of the entire five-day signal modelled in Fig. E.1(d). The sampling interval for all three sine signals was one hour to be consistent with the field measurements, and the signals for parts $A$ and $B$ were shifted relative to the tidal stage signal by 7 and 17 hours, respectively.
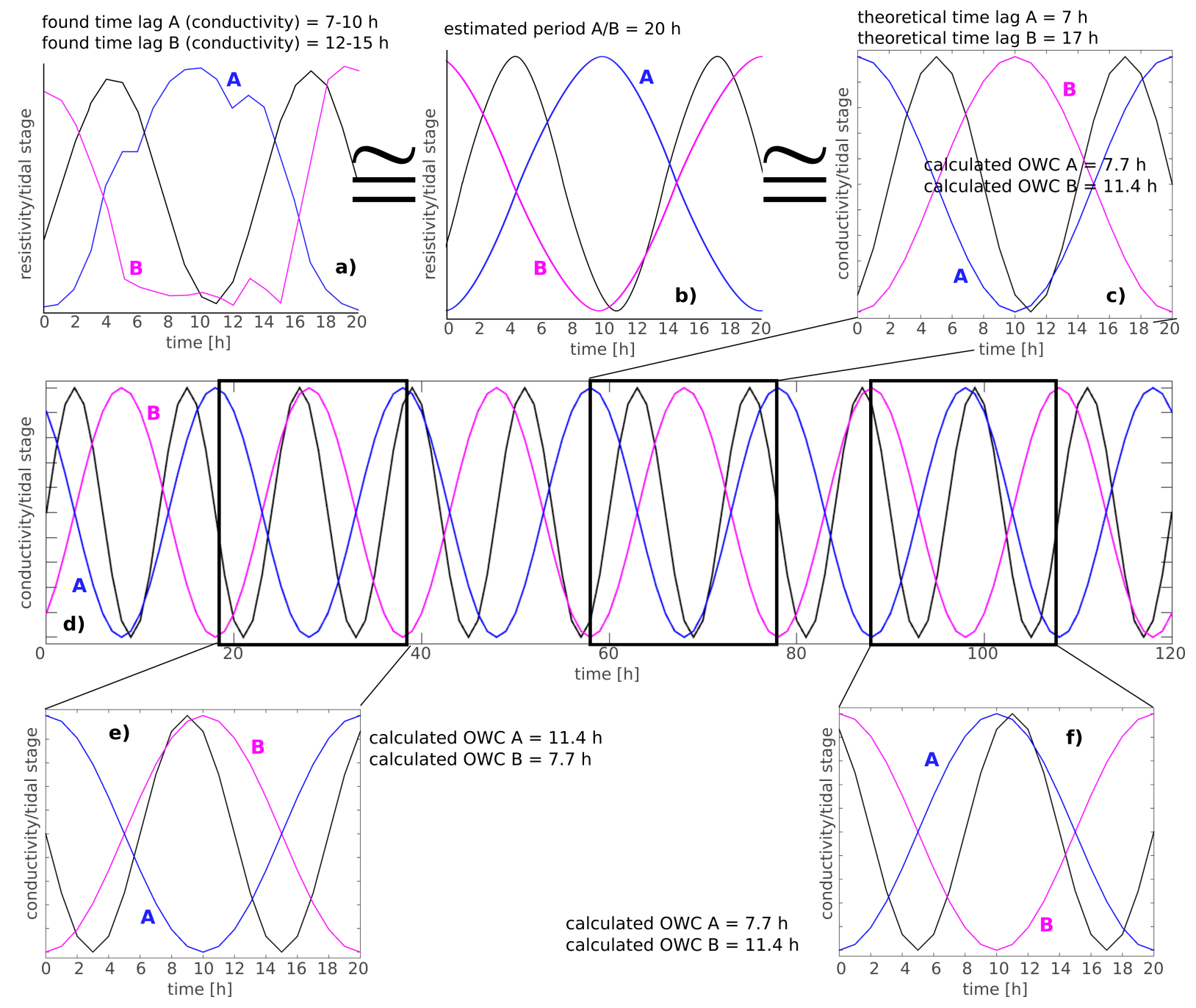

FIGURE E.1: Modelling of possible relationship between DC resistivity and tidal signals with a) two typical DC resistivity model cells for parts $A$ and $B$ plotted together with the tidal stage which approximate to about b) their sinusoidal representation from which a period of 20 hours for the DC resistivity signal was estimated, and used in c) to model electrical conductivity of those cells in order to find the same relationship between the three signals from d) which shows the signals for tidal stage with a period of 12 hours and the two inverse resistivity signals (i.e. conductivity) for $A$ and $B$ with periods of 20 hours and a time shift of 7 and 17 hours, respectively. However, as e) and d) show, there are several possibilities in a long time series for the time lags to occur in different variations.

Although, the relationship between the three signals displayed for a 20-hour window in Fig. E.1(c) resembles the field situation in Fig. E.1(a) and its approximation in Fig. E.1(b) reasonably well (note that the field signals are in resistivity, while the modelled signals are in 
conductivity for consistency with DTW analyses of the field data), it appears to highly depend on where in the five-day time series the monitoring window is placed in order to reproduce this situation (i.e. a shift of the window by five hours will show a different relationship). Therefore, even if this modelled situation represents the field situation well, it only gives a snap shot of a much larger signal with differing relationship characteristics over time. This can be illustrated for example when taking the 20-hour window between 19 and 29 hours as shown in Fig. E.1(e) where the conductivity signals are in the same position as in Fig. E.1(c), but the tidal signal is shifted by about four hours relative to them. This changes the temporal relationship of the tidal stage with parts $A$ and $B$ and results in the reversed OWC values found for these modelled time series (i.e. 7.7 hours for $A$ in Fig. E.1(c) and 11.4 hours for $A$ in Fig. E.1(e), and vice versa for $B)$. The reversed temporal relationship between the signals is a consequence of the differing periods of the tidal and resistivity signals.

Another example of this is illustrated with the window taken on the signal length between 89 and 109 hours (Fig. E.1(f)), where the mirrored situation of the original signal window is found. There, the tidal signal has the same temporal relationship with both signals $A$ and $B$ but all time series are shifted by $180^{\circ}$ compared to Fig. E.1(c). However, because for the DTW analysis global phase shifts do not matter as only the curve shapes are matched by stretching and dilatation along the time axis, this temporal snap shot leads to the same OWC as observed with the original modelling window between 59 and 79 hours.

In the above example, the OWC found between signals $A, B$ and the tide switches between $A$ and $B$ every 10 hours and repeats every 30 hours for a 20-hour window. This equals to 2.5 tidal and 1.5 resistivity/conductivity signal periods. If a window length of 30 hours is chosen instead, the OWC calculated from DTW for signals $A$ and $B$ are equal at 11.5 hours, which is close to the average of the two actual OWC. A 60-hour window is increasing the OWC to 21 hours for both signals. Therefore, having longer monitoring times appears not to be beneficial for DTW analysis, but only seems to increase the OWC instead of leading to a more accurate estimate. Choosing a monitoring length according to the resistivity signal period seems to be crucial in order to get an idea of OWC values between a tidal and tidally induced resistivity signal, as was implied also from the case where the same signal periods were used. Because of the importance of choosing an appropriate time window, DTW analyses between different monitoring data 
sets are probably hardly comparable if they were not collected with the exact same temporal dependencies of the different signals. This further suggests that from DTW analyses alone it is not possible to distinguish which parts $A$ or $B$ are hydraulically more or less conductive. This is because for example in the hypothetical case of monitoring the situation in Fig. E.1(e) in the field, one would actually label curves $A$ and $B$ the opposite way around (i.e. decreasing conductivity with increasing tide would be accounted to curve types $B$ (or 2) instead of $A$ ) in resistivity ratio plots. Hence, not only the OWC is dependent on the choice of the monitoring window, but also where regions $A$ and $B$ are ultimately identified. However, if the hydraulic conductivity of the individual subsurface parts could be found with other methods, such as for example pumping tests, it may be possible to extrapolate the information based on DTW analysis. 



\section{APPENDIX F}

\section{Estimate of resistivity periods from well groundwater table}

In order to find a period for a resistivity signal for the monitoring of an unconfined coastal aquifer it is assumed that the groundwater stage fluctuations in a nearby well can be taken as a proxy. From December 2015 until August 2016 a continuous groundwater stage and EC logger was installed in Te Horo Beach in well $R$ (Fig. 6.1), which taps into the shallow coastal aquifer at around mean sea level (-0.3 m.a.s.l.) and is, as estimated with Google Earth, about $207 \mathrm{~m}$ away from the mean high tide mark. Specific measurement parameters and the full data set from this well are discussed in Section 6.4.2. For determining the water table periods at this location, the water table data for two one-week monitoring periods were taken (i.e. closest possible to the November 2015 and the week of the February 2016 DC resistivity monitorings) to compare with the tidal stages during the same time span. The groundwater stage was smoothed using a moving average of five hours ( \pm 2 hours on each side of the measurement) and then de-trended and reduced to zero mean. Tidal stage data was calculated using the high tide and low tide information from the NIWA website in the same way as discussed in Section 5.2.3 on a one-hour interval and then interpolated to the sampling interval of $15 \mathrm{~min}$ of the well stage data. It is clear that this procedure for estimating a tidal signal completely ignores any effects from wind or atmospheric pressure on the seawater and therefore does not contain any other periodicity than the one from high and low tide, but since this is most likely the dominant period in the tidal signal, such effects should be negligible for the comparison with the groundwater data. Fig. F.1 
shows the tidal and groundwater stage data for the two measurement intervals in December 2015 and February 2016.

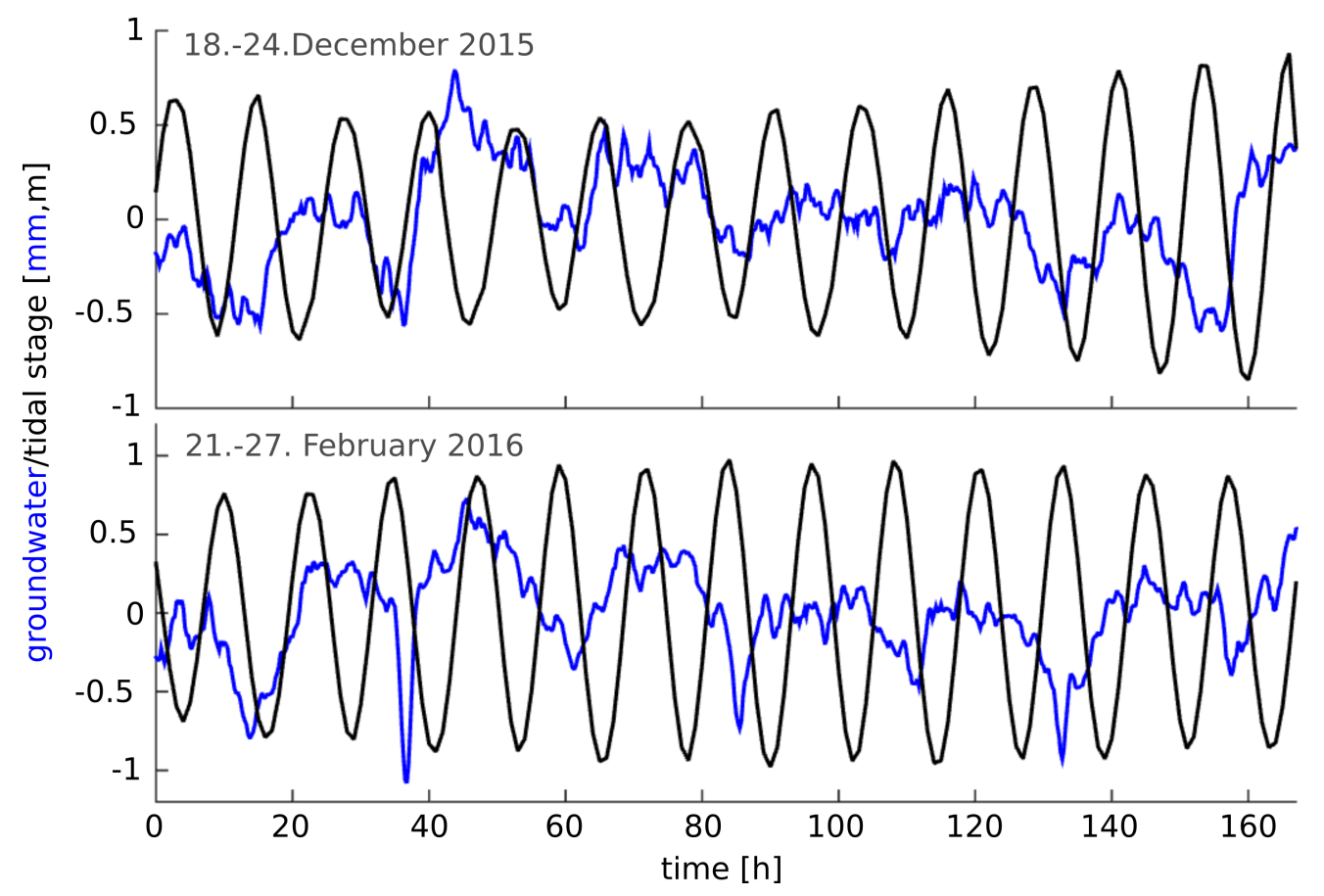

FIgURE F.1: Normalised groundwater table stage obtained from well $R$ (Fig. 6.1) in Te Horo Beach (blue) and tidal stage at Otaki River mouth (Fig. 2.1) (black) for two monitoring periods in December 2015 and February 2016 (168 hours, 7 days). Note the different units on the y-axis.

From plotting the two different stage signals together in Fig. F.1 it is already clear that the groundwater stage signal has a markedly longer period than the tidal stage signal. Both data sets have then been Fourier transformed in order to find the dominant periods of the groundwater and tidal stage signals. The result of this is shown in Fig. F.2 for the December 2015 and February 2016 monitoring periods. Although there is some variability in the derived periods, which is possibly due to temporal variability in the signals itself, it becomes quickly clear that there is the expected dominant 12 hour period visible for the tidal stage, whereas a higher period of approximately 21 hours shows the maximum amplitude for the groundwater stage signal during both monitoring windows. This confirms the possibility of having a longer period (i.e. 18-20 hours) variation for a resistivity time series relative to the tidal stage signal as was found from autocovariance for the Waikanae 21-hour monitoring in Chapter 5, and gives an estimate for the resistivity signal period in Te Horo as well. The minor peak in the groundwater stage time series indicating a period of the signal at around 11 hours is likely due to tidal activity, however, clearly not the dominant period. As the water table head is calculated from barometric 
information, minor peaks at 15 and 17 hours may be due to fluctuations in atmospheric pressure. A low amplitude peak of 24 and 21 hours, respectively, is additionally found in the tidal signal. These are most likely associated with the longer lunar and solar diurnal tidal signals.

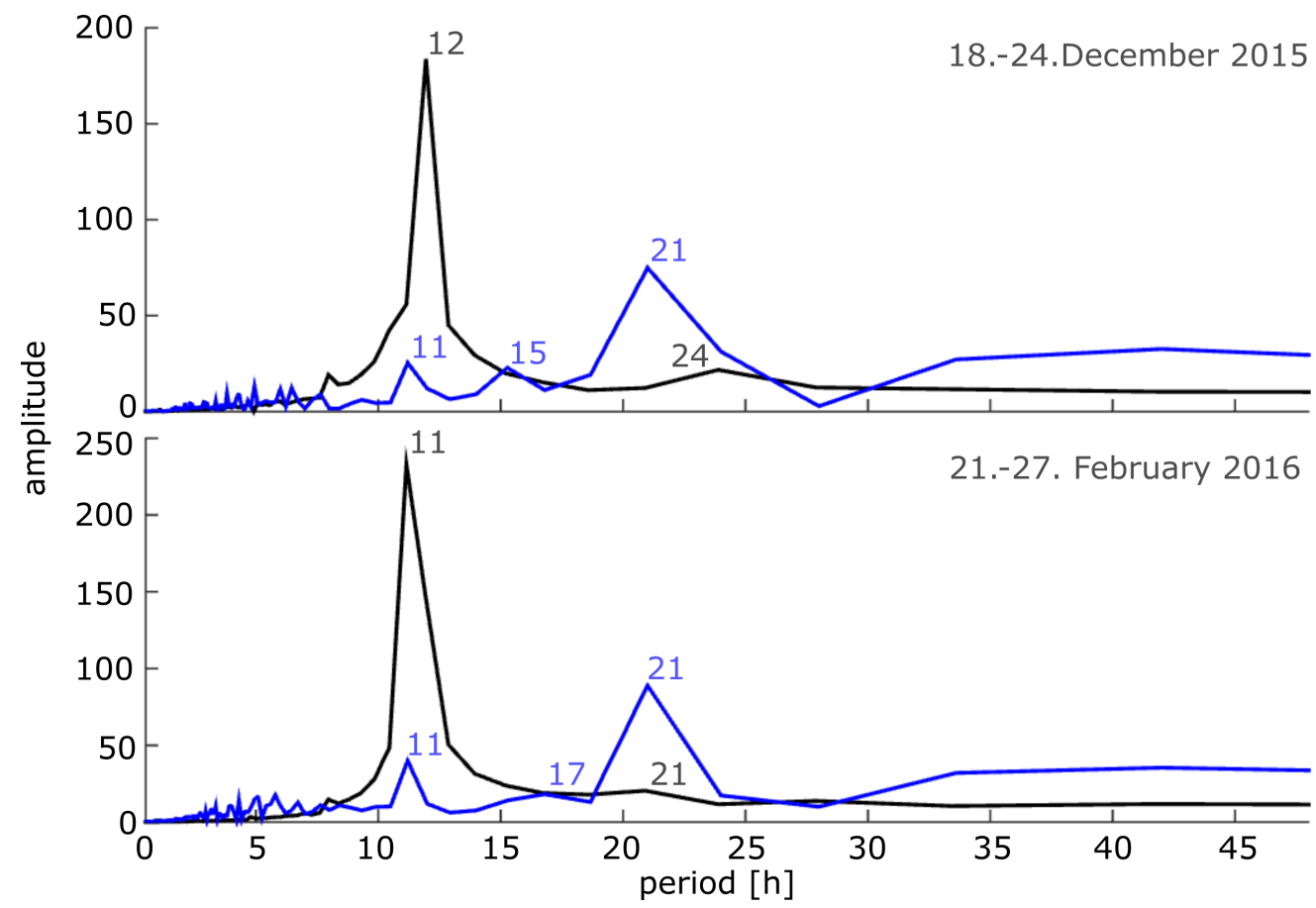

FIGURE F.2: Signal periods obtained from Fourier transforms for the groundwater (blue) and tidal (black) stage imaged in Fig. F.1 during two different times of the year. While the tidal stage shows a clear maximum amplitude at around 12 hours, the groundwater stage appears to have a main period of 21 hours. 



\section{APPENDIX $\mathbf{G}$}

\section{Groundwater chemistry - $\mathrm{pH}$ and dissolved oxygen relationships}

\section{G.1 pH relationships}

The $\mathrm{pH}$ (i.e. the acidity/alkalinity of the water - with $\mathrm{pH} 7$ being neutral, $\mathrm{pH}<7$ being acidic and $\mathrm{pH}>7$ being alkaline) of groundwater has a major influence on dissolution/coagulation processes of minerals and ions in solution. As illustrated in Fig. G.1, higher pH values are found in winter when the aquifer has lower temperatures. This makes sense as the formation of hydrogen $\left(\mathrm{H}^{+}\right)$and hydroxide $\left(\mathrm{OH}^{-}\right)$ions (which essentially determine acidity) from water absorbs heat (e.g. Hem, 1985). Therefore, if the temperature of the groundwater is increased water gets decomposed into hydrogen and hydroxide ions in order to maintain the equilibrium:

$$
H_{2} \mathrm{O}_{(l)} \rightleftharpoons H_{(a q)}^{+}+O H_{(a q)}^{-}
$$

This essentially means that there are more hydroxide ions available for chemical reactions with the rock/sediment matrix and pore fluid in an aquifer at higher temperatures.

Another important chemical reaction in an aquifer is the dissolution of calcium and magnesium ions from carbonates. In general, the lower the $\mathrm{pH}$ the more soluble are compounds such as calcium carbonate $\left(\mathrm{CaCO}_{3}\right)$ and magnesium carbonate $\left(\mathrm{MgCO}_{3}\right)$ and hence the larger the 


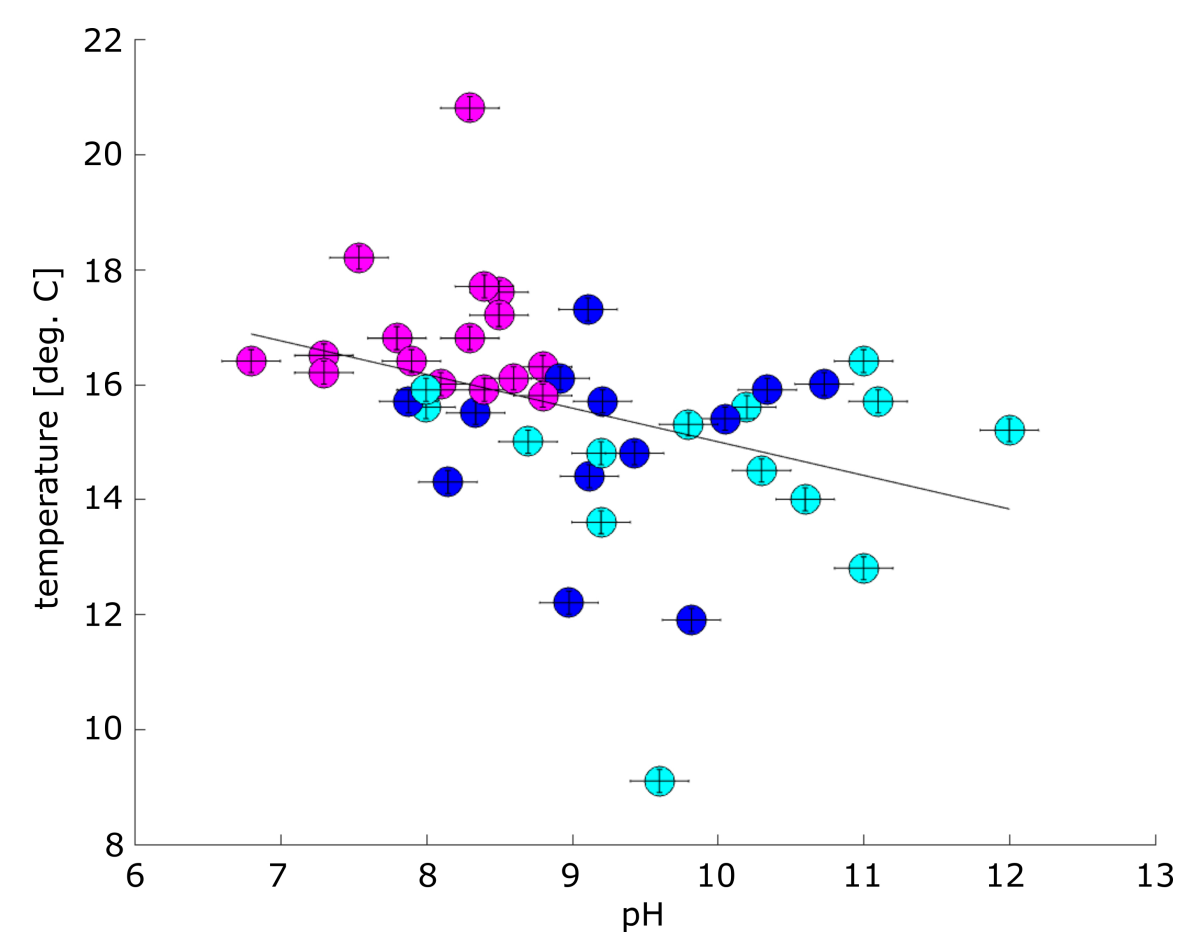

FIGURE G.1: Temperature vs. pH for all bores and samplings. A negative relationship is found between decreasing temperature and increasing $\mathrm{pH}$.

concentration of $\mathrm{Ca}$ and $\mathrm{Mg}$ ions in the water. The dissolution of carbonate rocks is initiated by carbon dioxide $\left(\mathrm{CO}_{2}\right)$ dissolving in the water:

$$
\mathrm{CO}_{2(g)}+\mathrm{H}_{2} \mathrm{O}_{(l)} \rightleftharpoons \mathrm{HCO}_{3(a q)}^{-}+\mathrm{H}_{(a q)}^{+}
$$

This releases hydrogen ions which lower the $\mathrm{pH}$ and react with $\mathrm{CaCO}_{3}$ or $\mathrm{MgCO}_{3}$ to release $\mathrm{Ca}$ and $\mathrm{Mg}$ ions, respectively, according to:

$$
\begin{gathered}
\mathrm{CaCO}_{3(s)}+2 \mathrm{H}_{(a q)}^{+} \rightleftharpoons \mathrm{Ca}_{(a q)}^{2+}+\mathrm{CO}_{2(g)}+\mathrm{H}_{2} \mathrm{O}_{(l)} \\
\mathrm{MgCO}_{3(s)}+2 \mathrm{H}_{(a q)}^{+} \rightleftharpoons \mathrm{Mg}(a q) \\
2+
\end{gathered}
$$

In Fig. G.2 a generally negative trend between $\mathrm{pH}$ and the concentrations of $\mathrm{Mg}$ (and to a lesser extent for $\mathrm{Ca}$ ) ions can be observed during winter, confirming that the more $\mathrm{Ca}$ and $\mathrm{Mg}$ dissolved in the water, the lower the measured $\mathrm{pH}$ of the groundwater is. This could be attributed to the fact that in winter the flowthrough of freshwater provides a continual supply of $\mathrm{CO}_{2}$ dissolved in water which lowers the $\mathrm{pH}$ at the same time as increasing the concentration of $\mathrm{Ca}$ and $\mathrm{Mg}$ ions. However, during the summer period the concentration of these two ions is 
much more constant and the $\mathrm{pH}$ is generally lower. This may result from a lessened supply of freshwater, and hence $\mathrm{CO}_{2}$, therefore having a main dependency of $\mathrm{pH}$ from temperature. This means that $\mathrm{Ca}$ and $\mathrm{Mg}$ concentrations are essentially independent of $\mathrm{pH}$ during summer. An observed increase of Ca and Mg ions during summer 2016, as for example observed in bores 2, 11 and 15 (Fig. 6.6), therefore is more likely due to exchange reactions initiated by seawater intrusion. On the other hand, bores that show an increase of $\mathrm{Ca}$ and $\mathrm{Mg}$ during winter time (e.g. 4, 7, 9, 10 and 12) may be more influenced by the dissolution of carbonates, as explained above, as a consequence of a higher throughflow of freshwater containing more $\mathrm{CO}_{2}$.
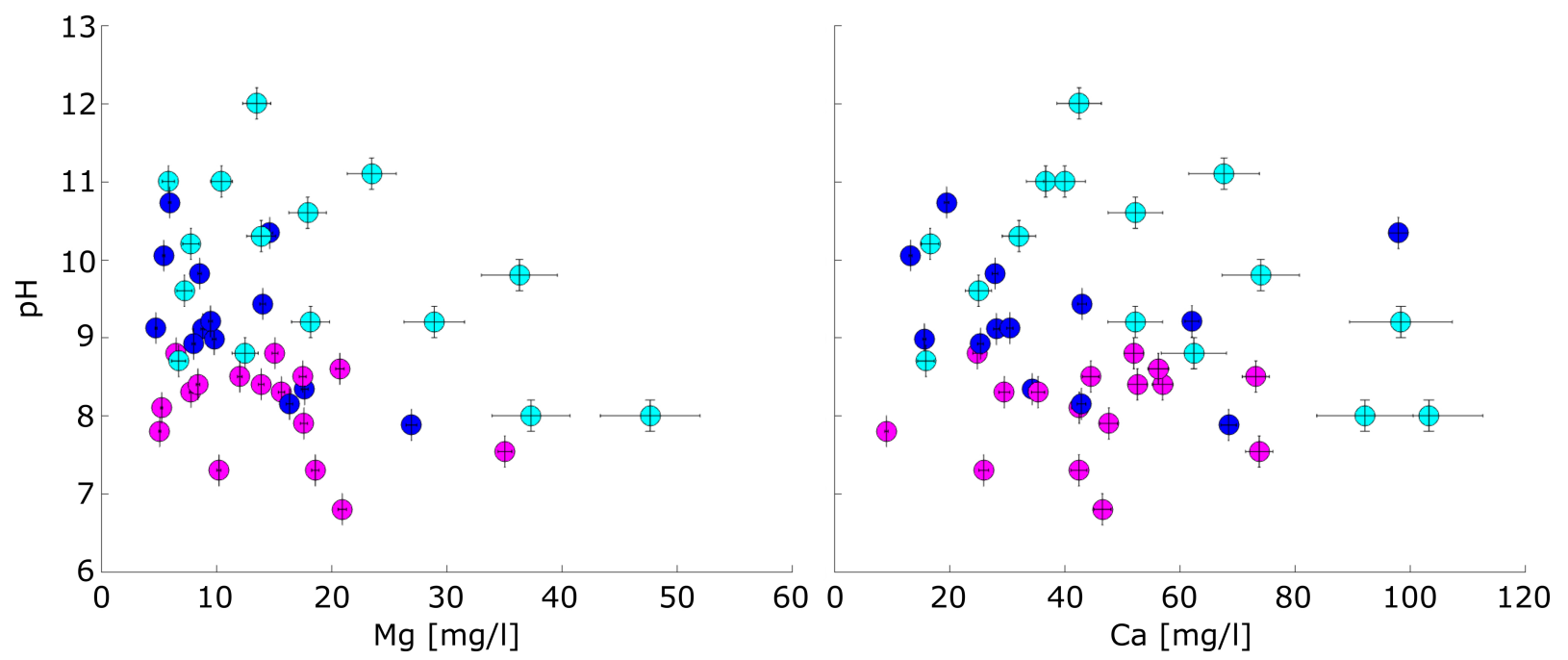

FIGURE G.2: pH of bore samples taken three times between June 2015 and August 2016 in the Te Horo Beach township plotted against the major cations magnesium and calcium. The $\mathrm{pH}$ shows seasonal dependency for all bores.

Figure G. 3 shows the manganese concentrations measured in the June 2015 sampling against their $\mathrm{pH}$ values. An exponential increase in $\mathrm{Mn}$ with decreasing $\mathrm{pH}$ is inferred from this data set with bore 3 being on the high-Mn concentration end. As the $\mathrm{pH}$ for this bore is relatively neutral (7.8), reducing conditions would be expected in order to obtain Mn concentrations of the observed magnitude (British Geological Survey, 2003). Reducing conditions are the main reason for the accumulation of manganese and iron, but the regional occurrence of these trace metals can also be regulated by the differing kinetics of the two ions (oxidation of $\mathrm{Mn}$ is much slower than for $\mathrm{Fe}$, which means that Mn concentrations can be much higher in anaerobic systems) (British Geological Survey, 2003). Anaerobic and aerobic conditions of the aquifer are discussed in terms of dissolved oxygen (DO) concentrations the following section. 


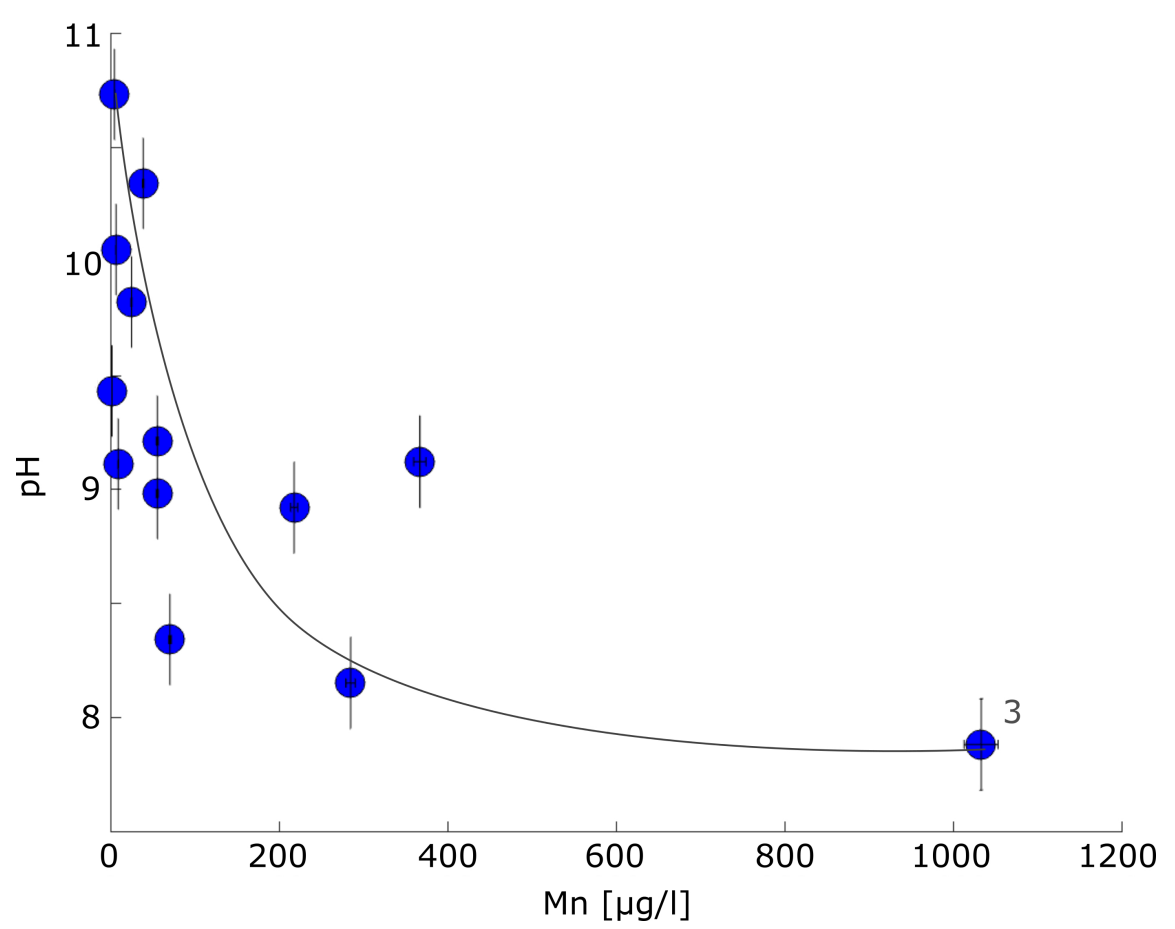

FIGURE G.3: Manganese in relation to $\mathrm{pH}$ for groundwater samples taken in winter 2015. From this it seems as decreasing $\mathrm{pH}$ conditions lead to exponentially increasing Mn concentrations in the shallow aquifer. However, note that there is no Mn data available for other samplings which would be necessary to reliably confirm such a behaviour.

\section{G.2 Dissolved oxygen relationships}

Three major factors exert some control on the total dissolved solids content in groundwater - temperature, $\mathrm{pH}$ and the redox potential of the aquifer. The latter defines the solubility of ions such as iron and manganese and may depend on the amount of oxygen available in the water. The existence of a more oxidising environment means that ions tend to go out of solution. Whereas reducing aquifer conditions will lead to an increased amount of ions in the groundwater. Dissolved oxygen (DO) concentrations depend on temperature, with lower temperatures having higher DO values. This is illustrated in Fig. G.4 for the references seawater (S) and Mangonoe stream water $(\mathrm{M})$ which show a decrease in DO with increasing temperatures between winter (w) and summer (s) in 2016. The seasonal change in DO is larger for the stream water but is quite small for the seawater. Also, freshwater, represented by the Mangaone stream, shows an approximately $20 \%$ higher DO content than seawater in winter, whereas during summer the difference is only about $5 \%$ and higher for seawater. Bores 1, 12, 14 and 15 also show this trend. Bore 8 shows the same trend with exception of its winter 2015 measurement which is unusual low for the temperature it was measured at. In general, the June 2015 measurements 
are suspiciously stable for all bores and it may therefore be possible that there was a systematic error with the sensor giving misleading DO readings. Two bores (2 and 4) show the reverse behaviour showing decreasing DO values with decreasing temperature, in contrast to the fact that cold water can hold more DO than warm water. This might also indicate measurement errors in DO at these locations, possibly due to the lack of a hose attached to the outside tap, thereby allowing the water to enrich in oxygen from the air between tap and sampling bucket for the summer 2016 measurements at these locations. All other bores have a very low DO concentration ( $<2 \mathrm{mg} / \mathrm{l}$ and an average of $0.5 \mathrm{mg} / \mathrm{l})$ year round, hence, indicating reducing (anaerobic) conditions for most of the shallow aquifer at the sampling sites is in agreement with the observation made by Hughes (1998) for the Te Horo Beach township based on measurements of nitrate- $\mathrm{N}$ and ammonia- $\mathrm{N}$ in various bores. There seems to be no relationship between bore depths and DO concentrations, and Hughes (1998) inferred the reducing environment of the shallow aquifer to be due to the high organic content in the lithology, which is likely to have a relatively heterogeneous distribution. High iron concentrations have only been found in bore 11, whereas there were elevated manganese concentration measured in bores 3 and 11. Both of these bores show groundwater depleted in oxygen, providing an environment for enhanced Fe and Mn solution. Low Fe concentrations but high Mn contents in the water measured in bore 3 may be explained by a generally low Fe content in the groundwater at locations away from the Mangaone stream, which appears to be the source of the iron in the water.
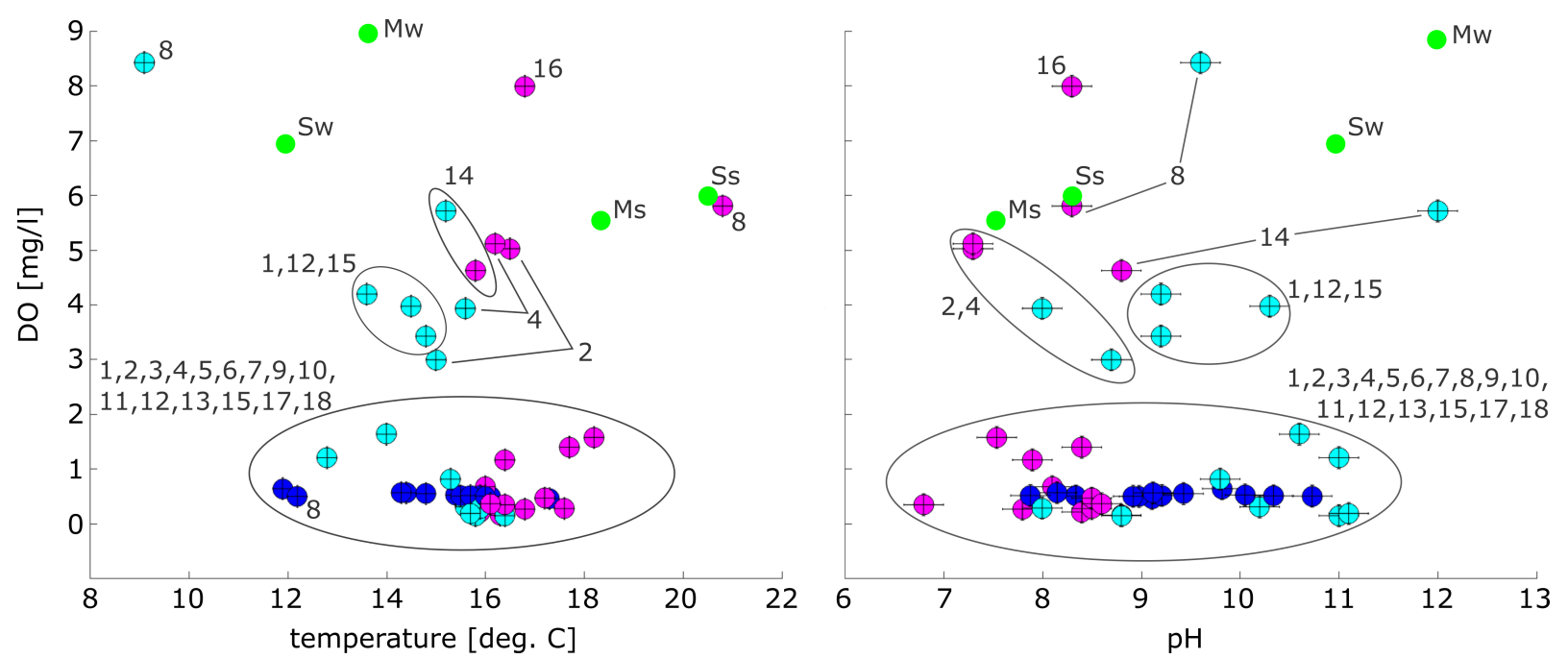

FIGURE G.4: Dissolved oxygen (DO) in relation to temperature and $\mathrm{pH}$ for all measurements carried out in the sampling period. Green dots mark summer (s) and winter (w) values for measurements taken in the seawater (S) and Mangaone stream (M) for reference. 
There seems to be no direct relationship of DO with $\mathrm{pH}$ for the majority of the bore samples in Fig. G.4. However, bores with DO values above $2 \mathrm{mg} / 1$ do suggest a linear relationship between $\mathrm{DO}$ and $\mathrm{pH}$ where an increasing $\mathrm{pH}$ results in more oxygen being dissolved in the groundwater. This makes sense given that a higher $\mathrm{pH}$ will shift the system towards an oxidising state where more oxygen is released into the water, as for example discussed in Zang et al. (2011). Again, bores 2 and 4 show the contrary behaviour, and as the $\mathrm{pH}$ was measured with a different device than DO and temperature, this is an additional indication for faulty DO measurements for these bores. The negative correlation between temperature and pH as implied by Fig. G.4 was already discussed with Fig. G.1 in Section G.1. 
APPENDIX $\mathbf{H}$

Additional seasonal DC resistivity data 


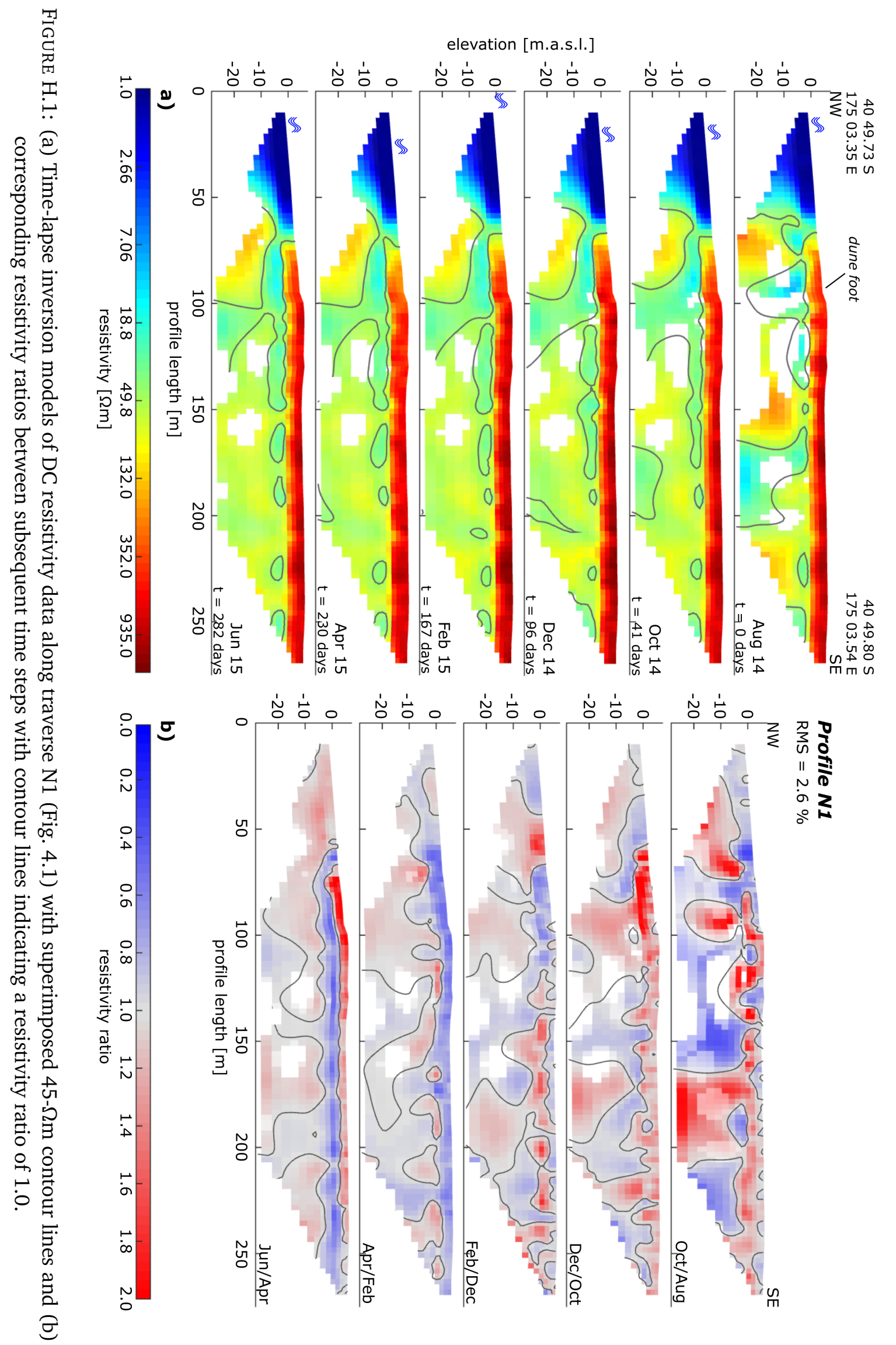




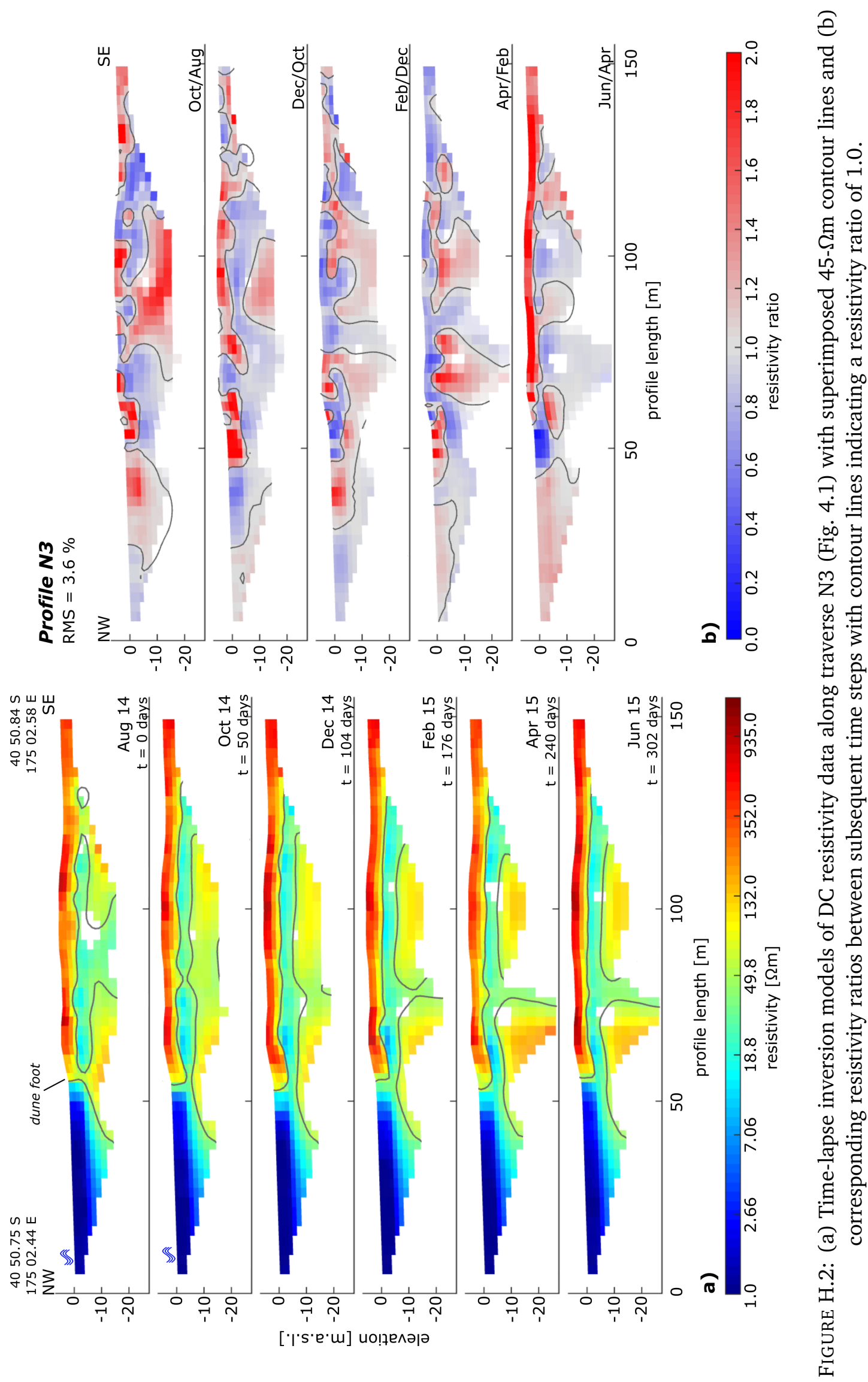




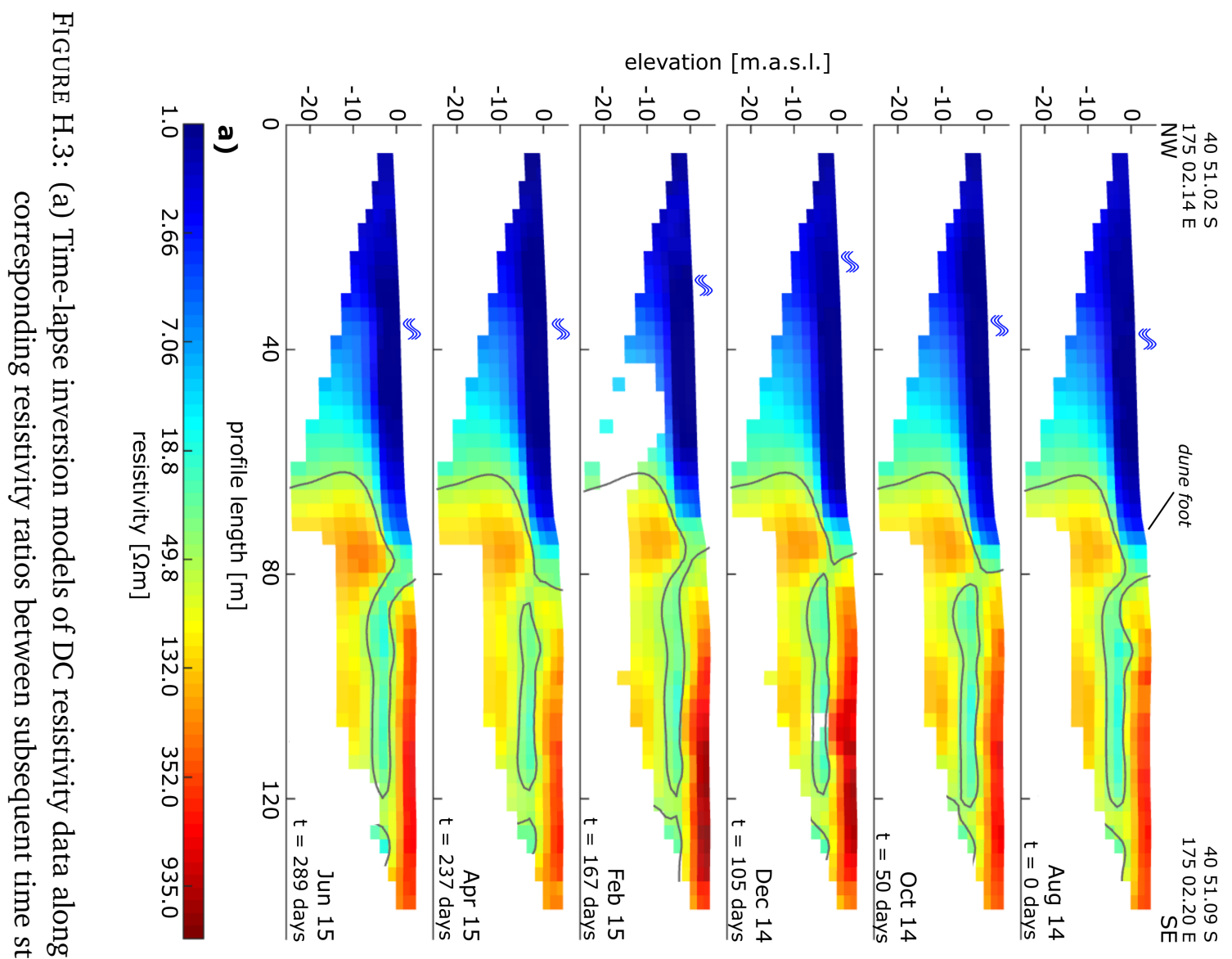

密

$\sum_{\substack{0 \\ 0}}$

官

8

要需

实范

究.

苞.

营.

志

.

突. 市

桴

芯. 岕

용
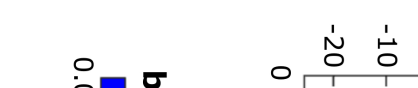

\section{年}

\section{(5)}

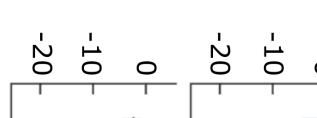

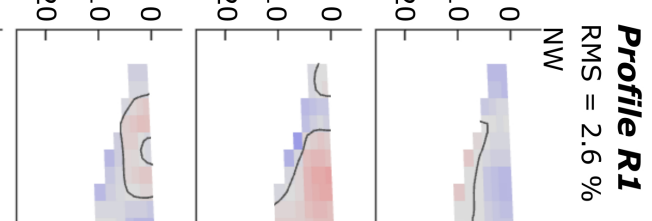

.

i

$\stackrel{\circ}{+}$

के

勇

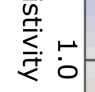

竞.

D

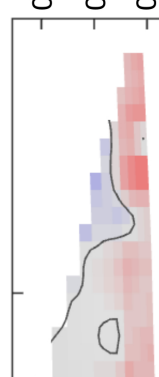

뭉
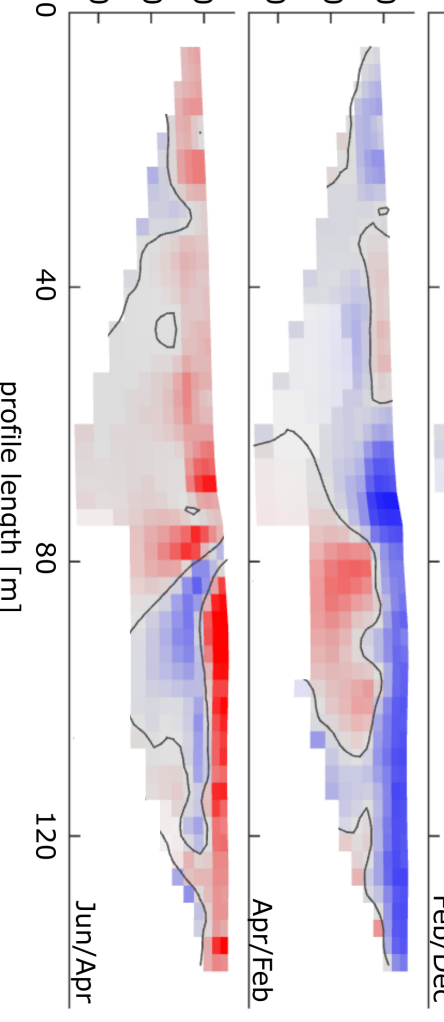

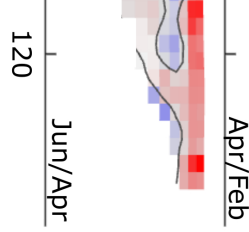

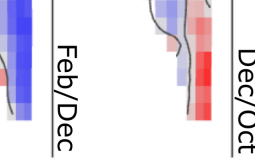

\section{策 \\ वर}



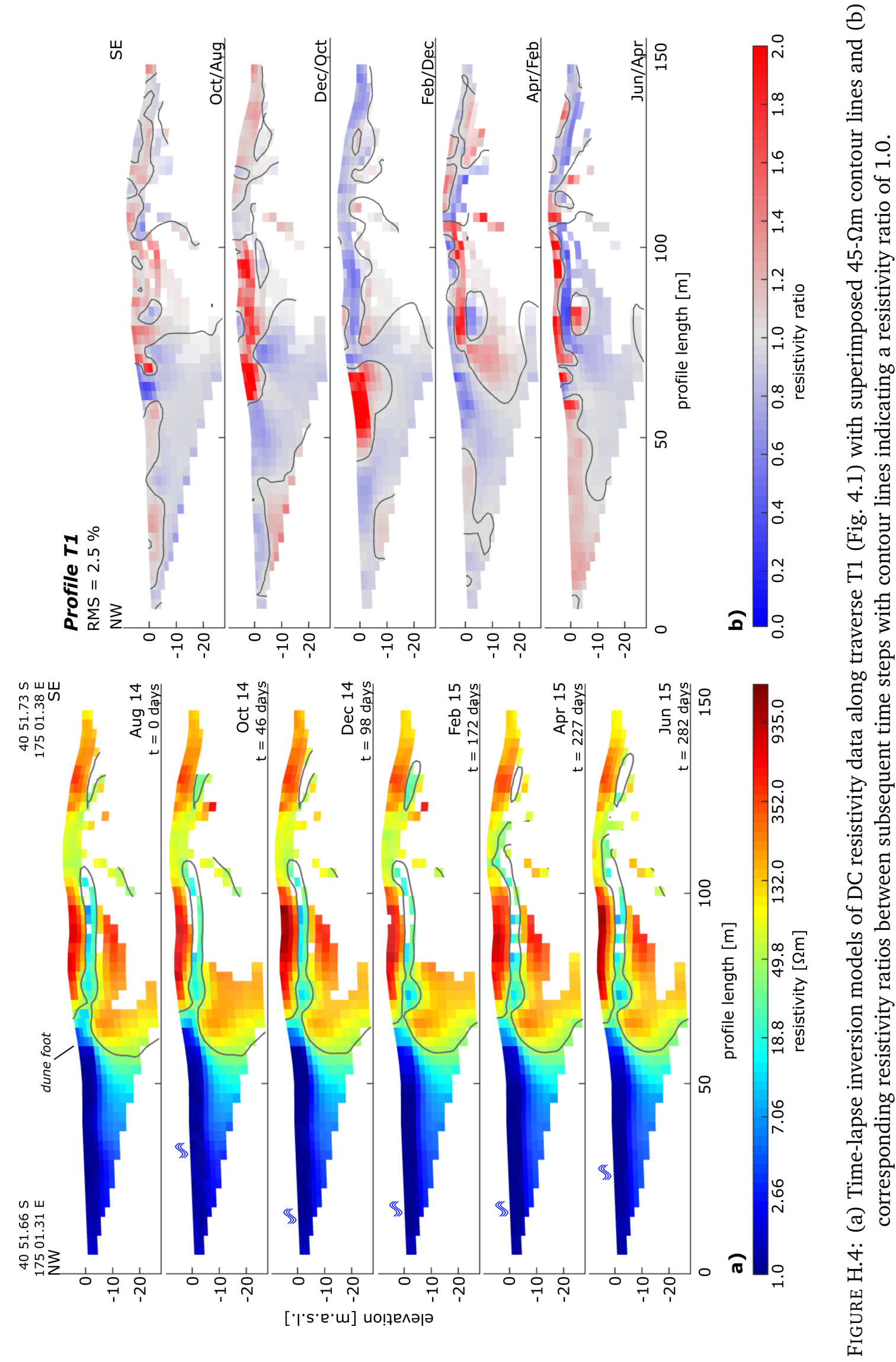


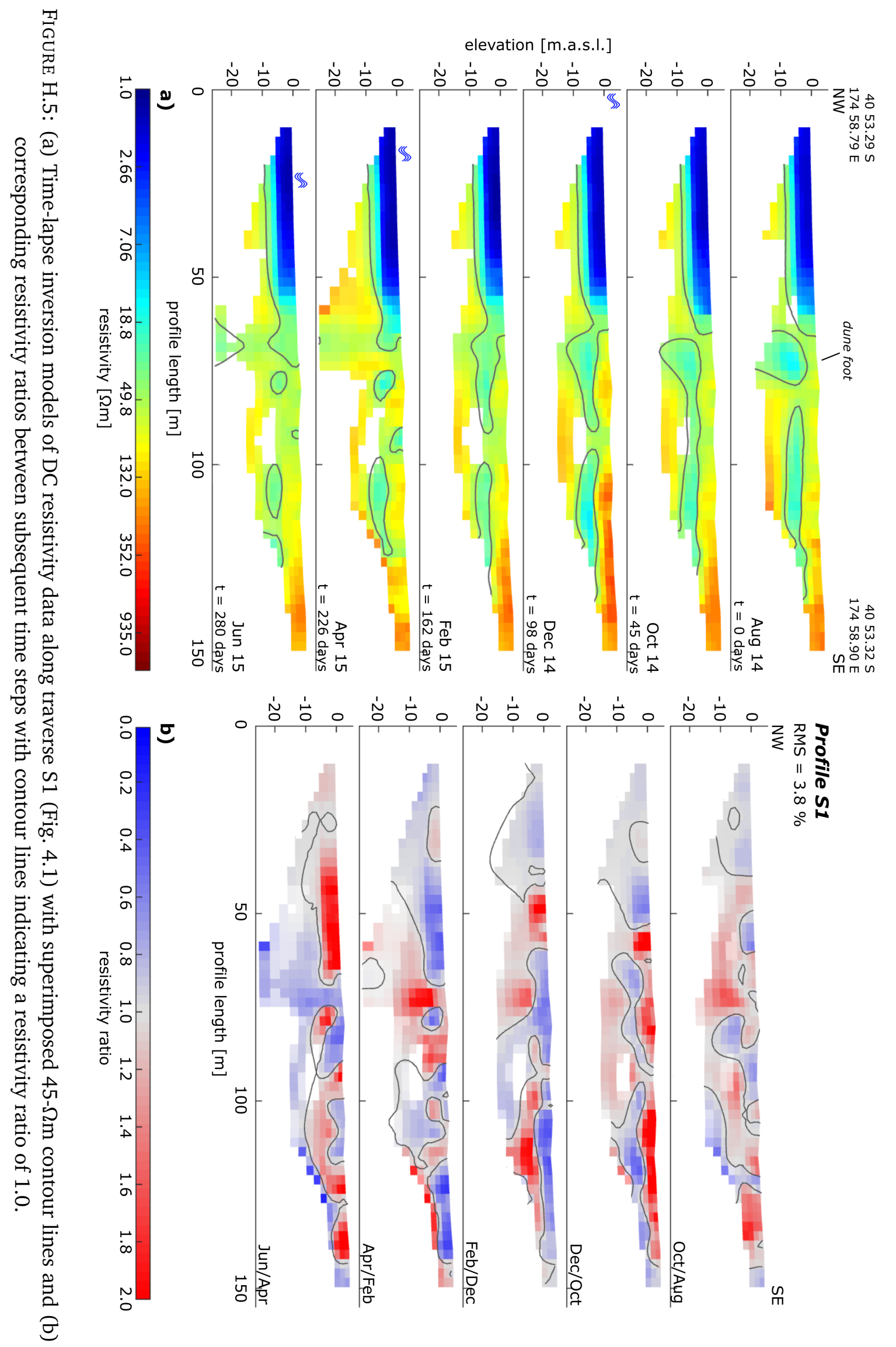




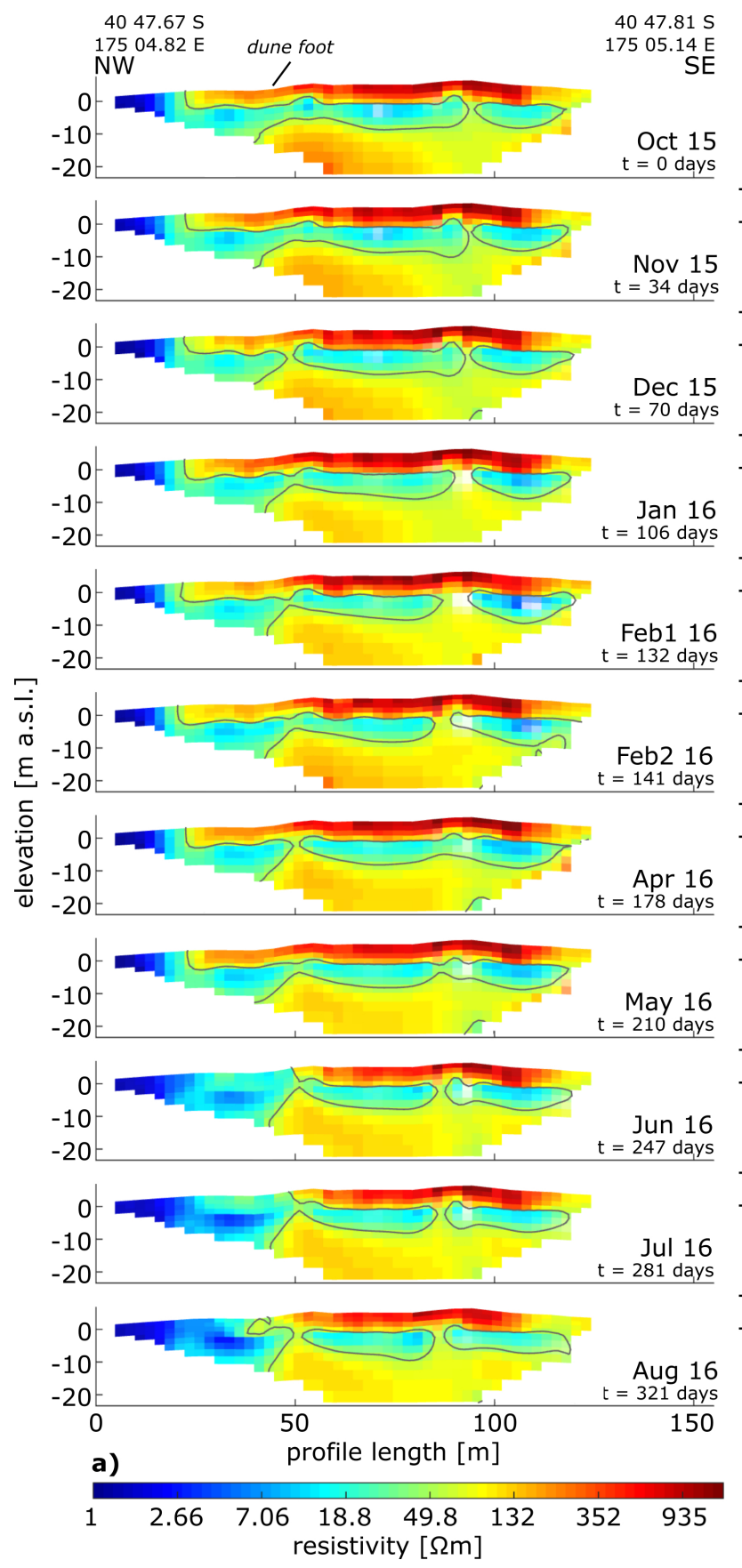

Profile TH2 RMS $=4.2 \%$
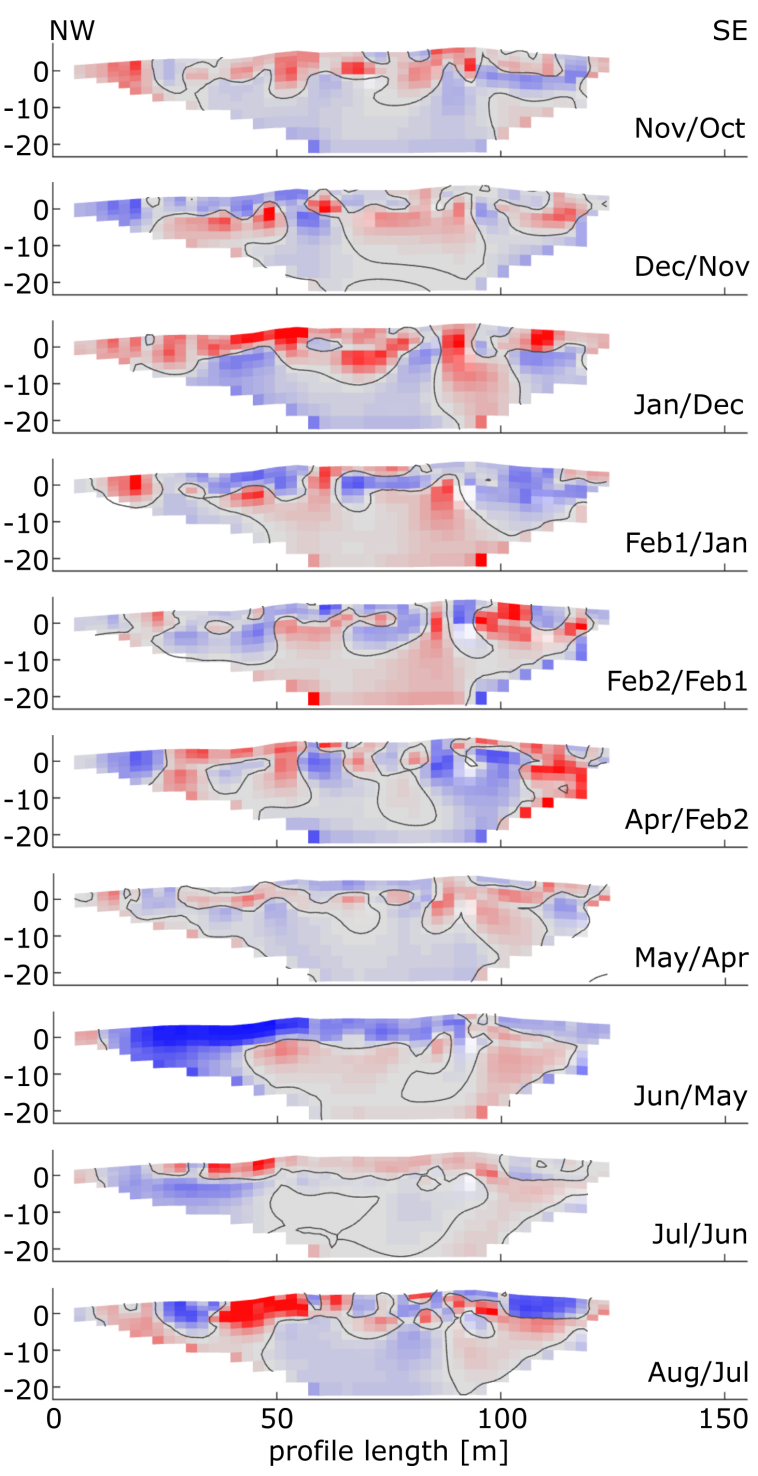

b)

$\begin{array}{lllllllllll}0.0 & 0.2 & 0.4 & 0.6 & 0.8 & 1.0 & 1.2 & 1.4 & 1.6 & 1.8 & 2.0\end{array}$

FIGURE H.6: (a) Time-lapse inversion models of DC resistivity data along traverse TH2 (Fig. 4.2) with superimposed $45-\Omega \mathrm{m}$ contour lines and (b) corresponding resistivity ratios between subsequent time steps with contour lines indicating a resistivity ratio of 1.0. 


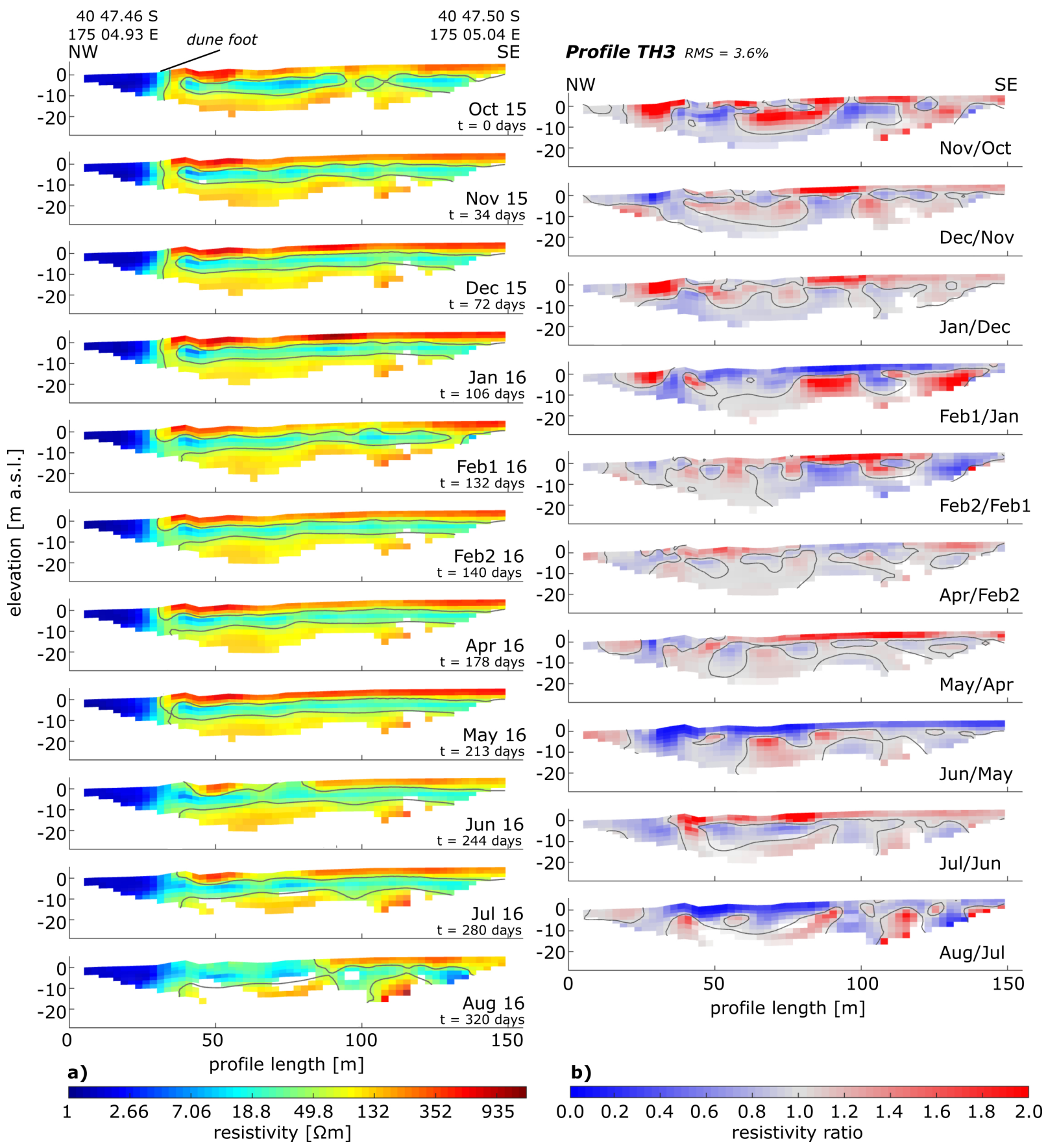

FIGURE H.7: (a) Time-lapse inversion models of DC resistivity data along traverse TH3 (Fig. 4.2) with superimposed $45-\Omega \mathrm{m}$ contour lines and (b) corresponding resistivity ratios between subsequent time steps with contour lines indicating a resistivity ratio of 1.0. 
APPENDIX I

YSI sensor results and ICP-MS analyses 

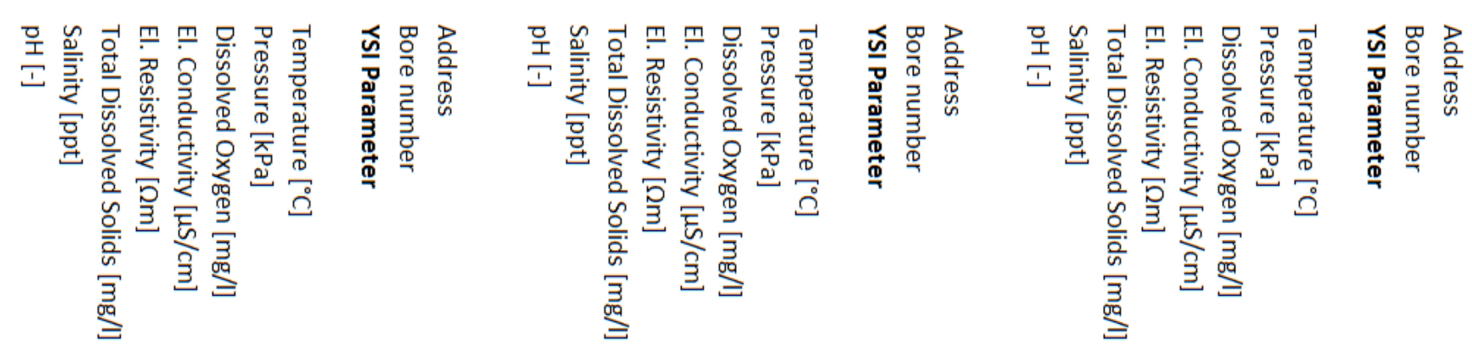

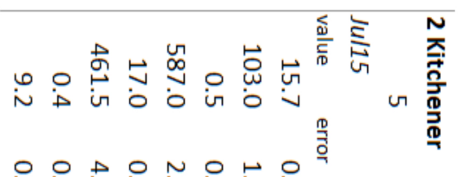

ปั

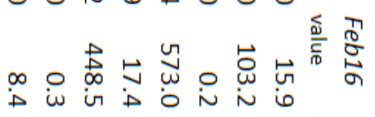

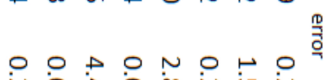

ปั

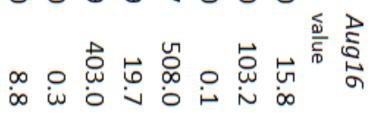

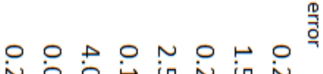

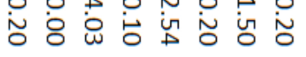

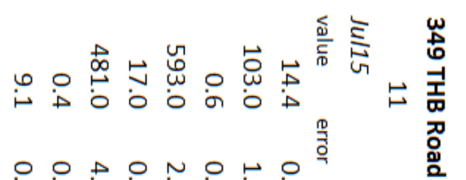

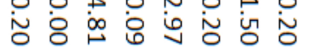

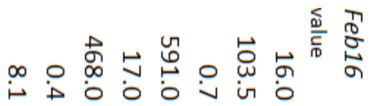

o.

ஸ்

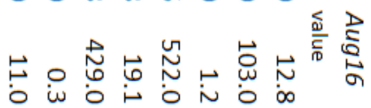

$\circ \circ \rightarrow 0 n \circ \leftarrow 0^{\circ}$

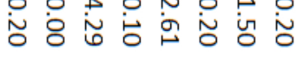

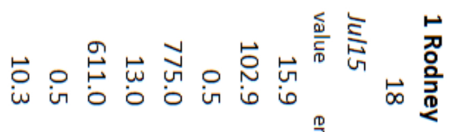

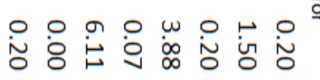

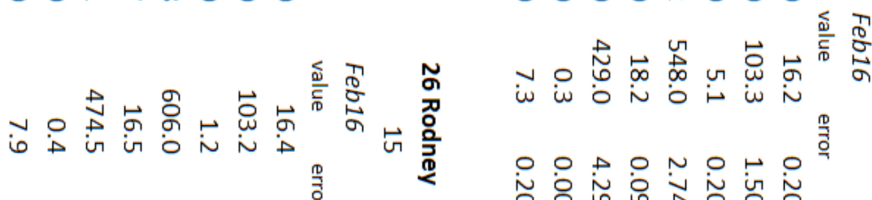

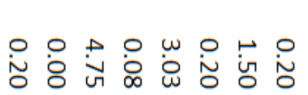

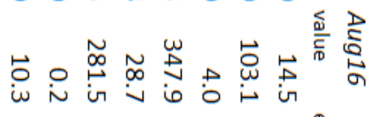

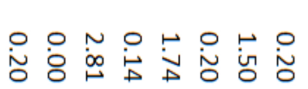

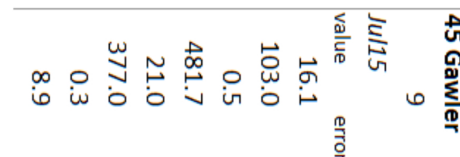

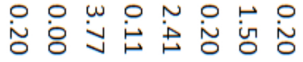

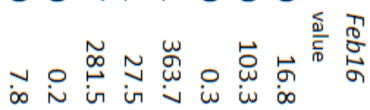

o. nor

ஸ்

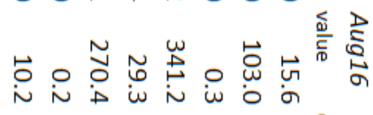

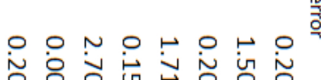

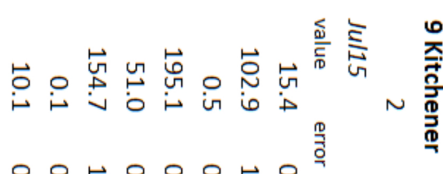

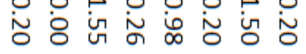

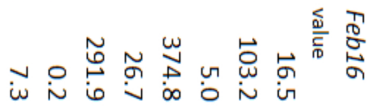

o. nor.hor

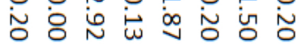

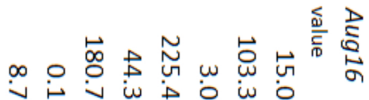

웡

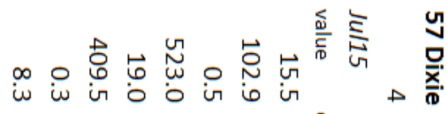

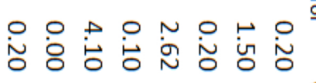

웡

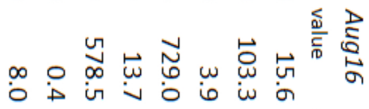

웡

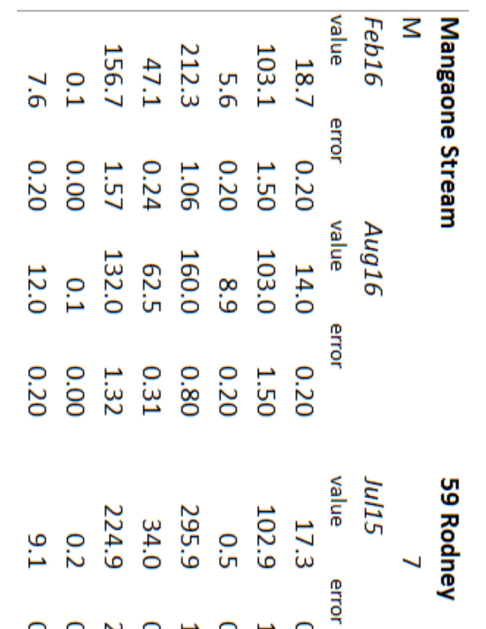

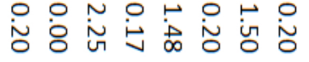

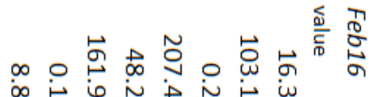

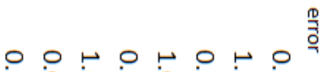

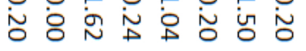

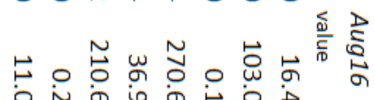

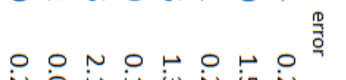

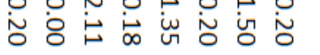

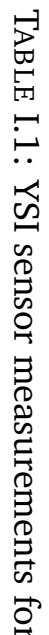

क्ञ

等 $w$ w

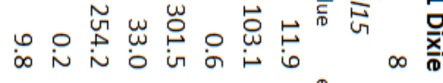

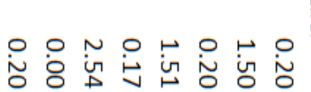

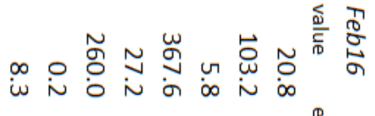

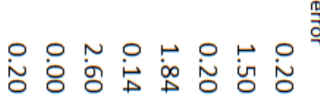

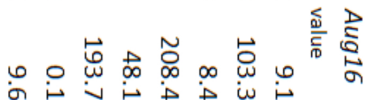

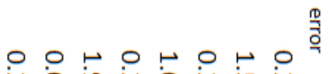

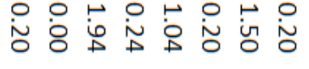

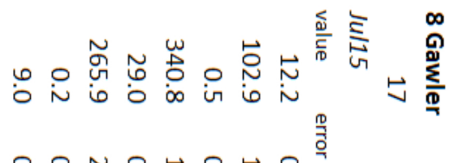

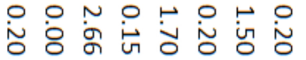



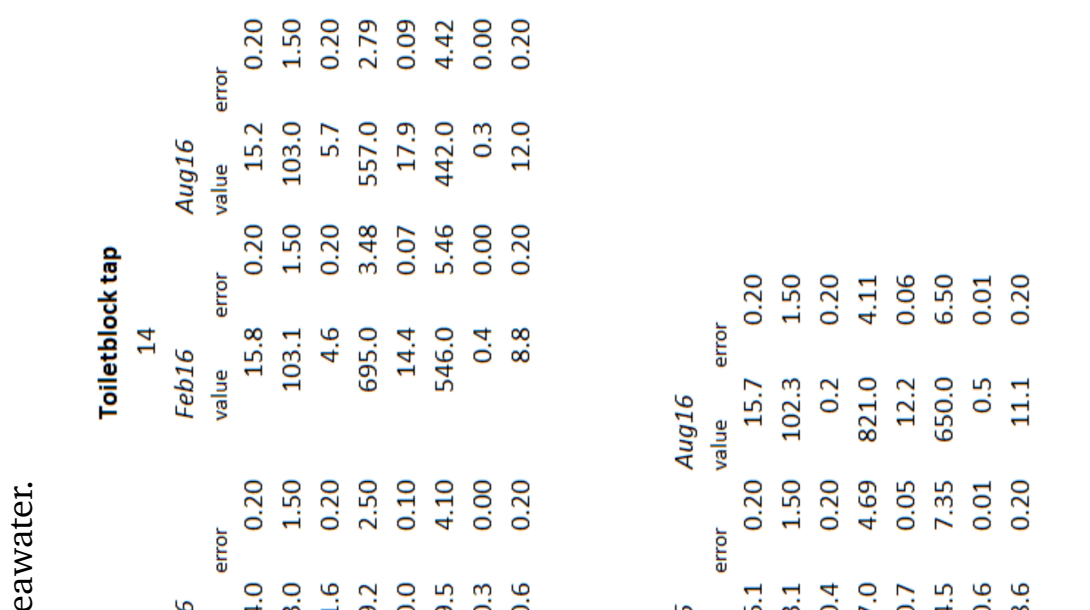

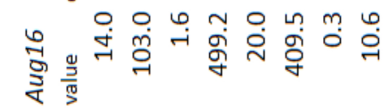

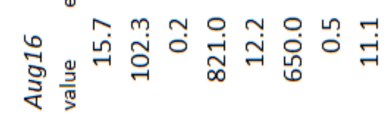

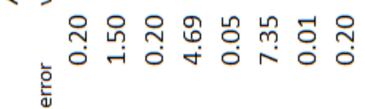

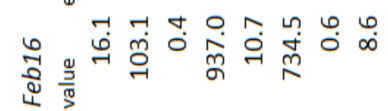
ฮี

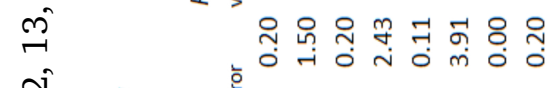

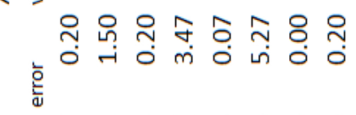

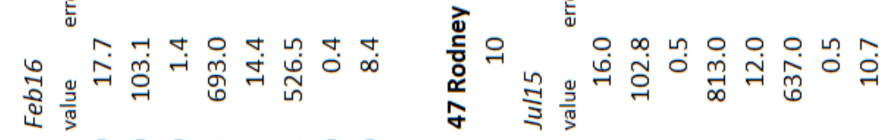

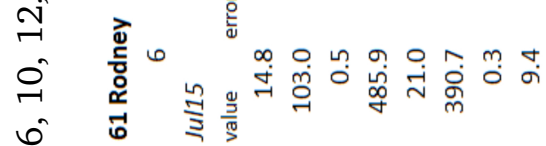
m

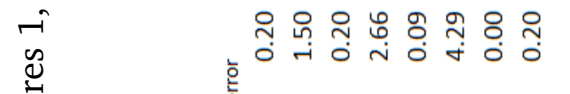

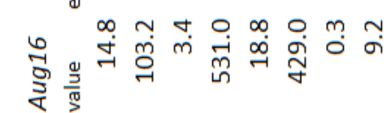

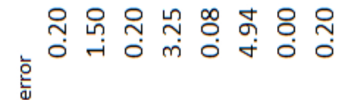

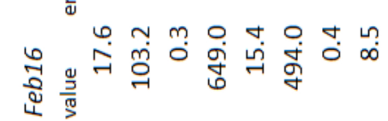

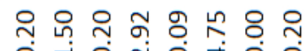

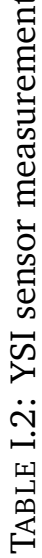

步

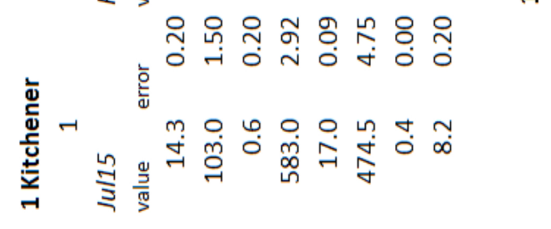

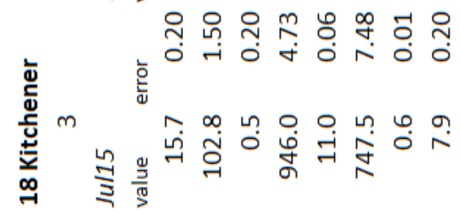

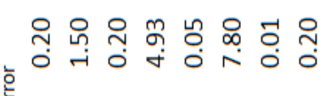
矛政

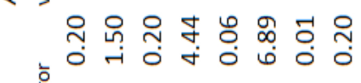
홍

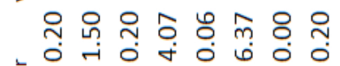

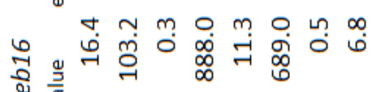

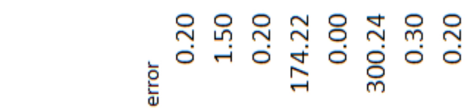

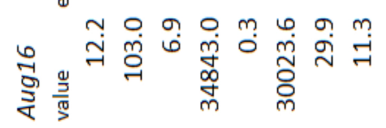
के अे

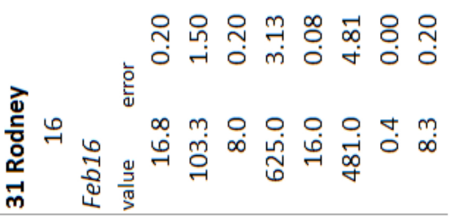

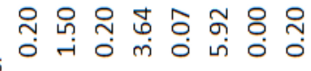
홓

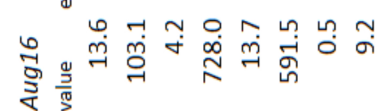

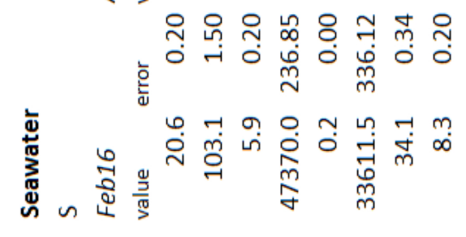

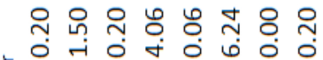

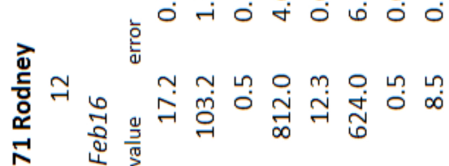

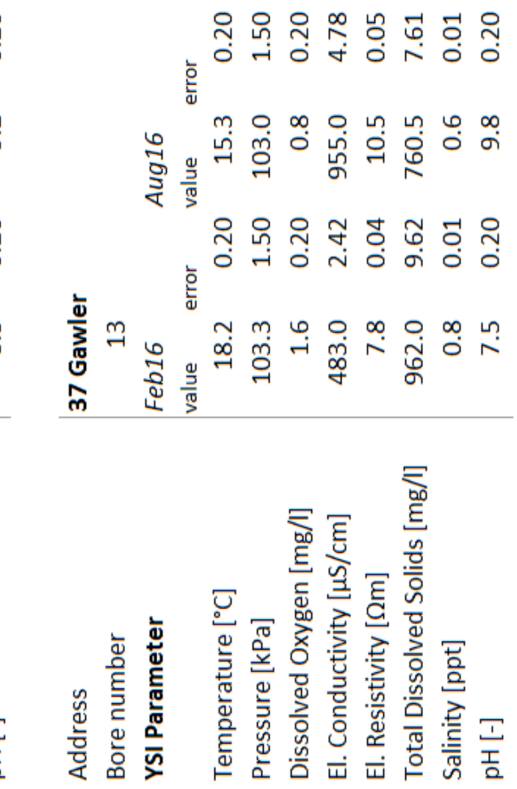




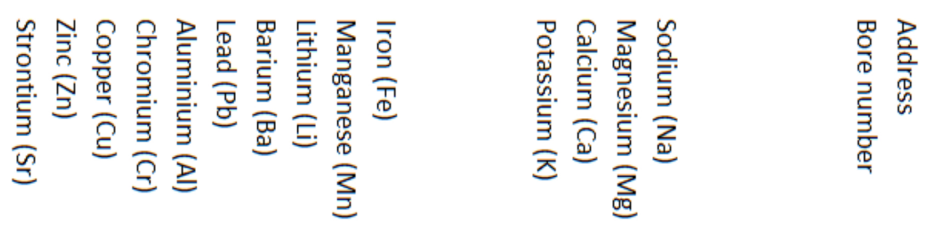

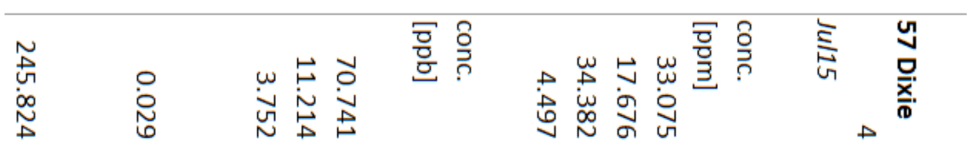

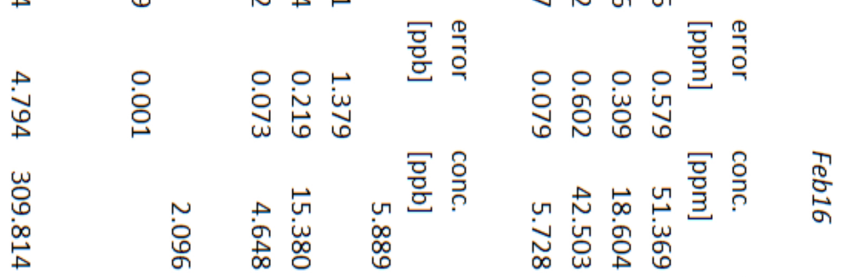

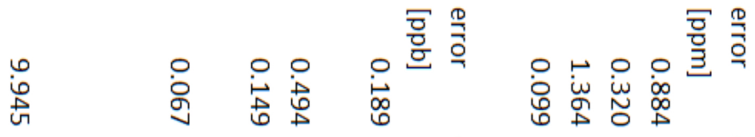

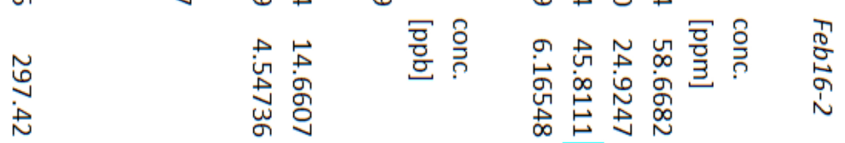

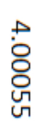

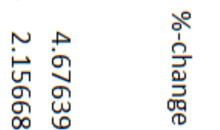

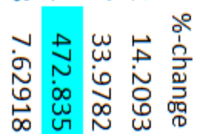

吕

का एँ

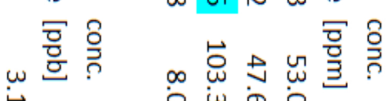

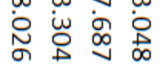

育

㨷

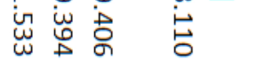

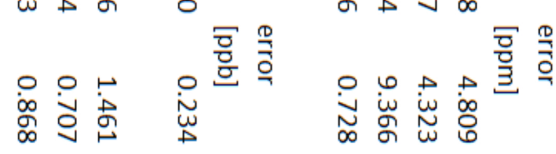

离

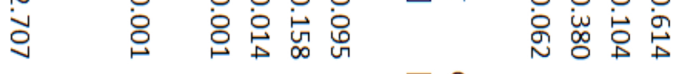

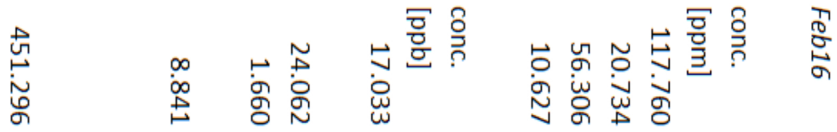

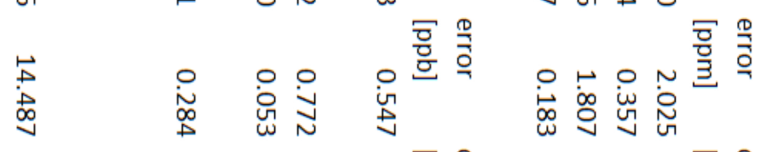

商

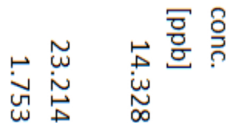

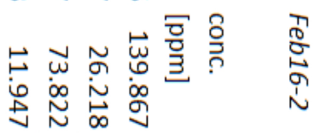

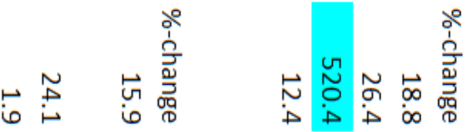

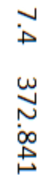

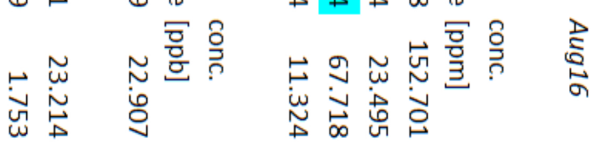

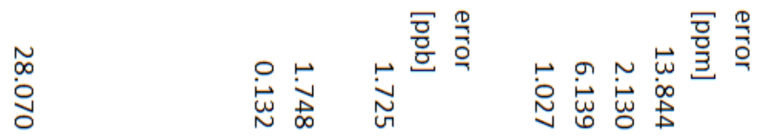




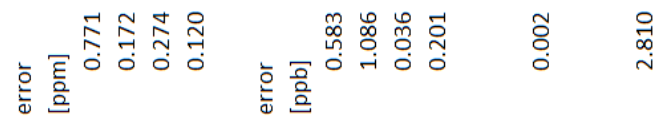

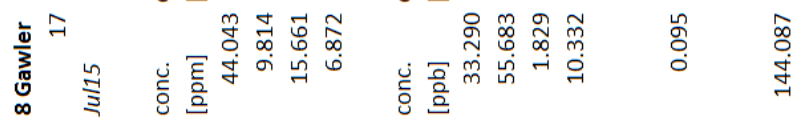

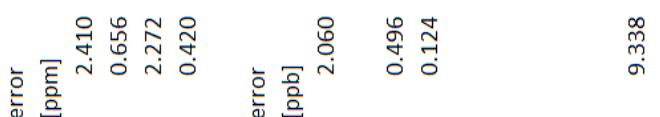

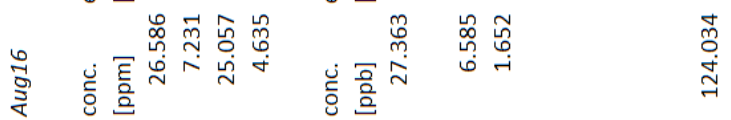

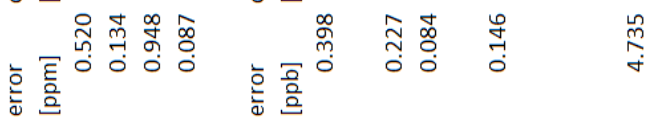

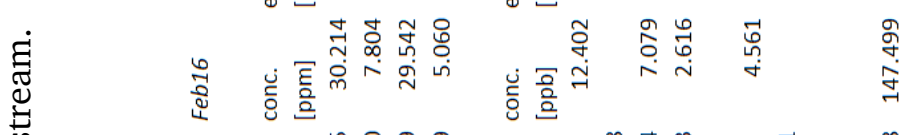

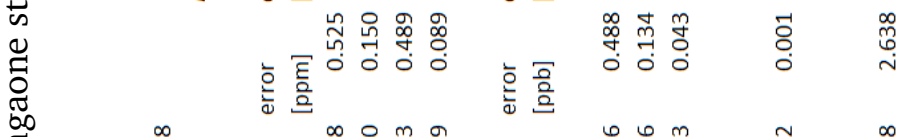

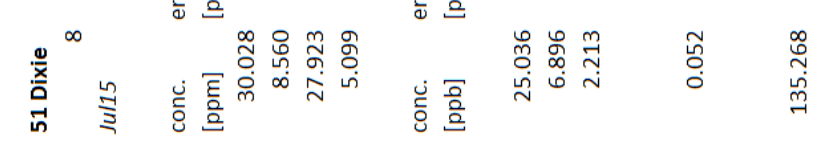

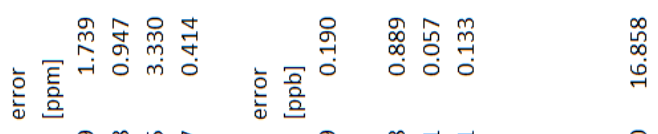

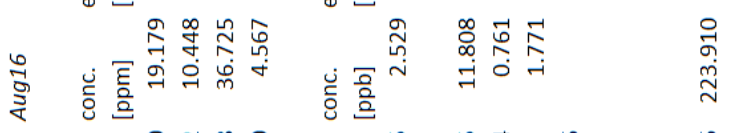

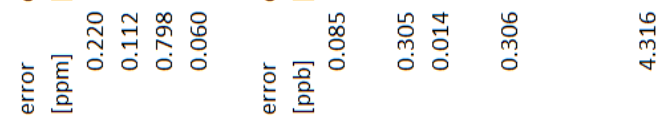

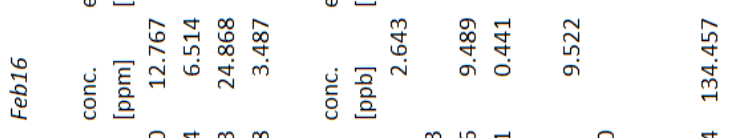

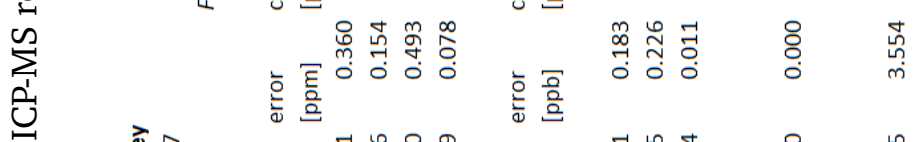

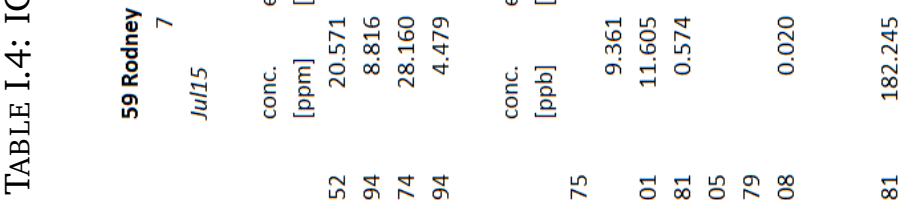

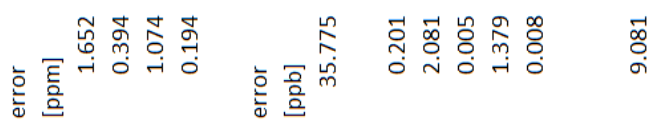

$$
\begin{aligned}
& \text { 岁 }
\end{aligned}
$$

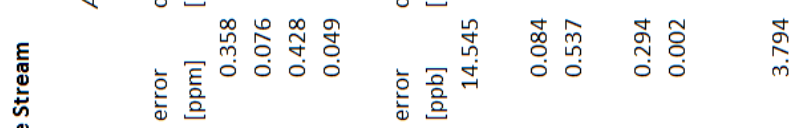

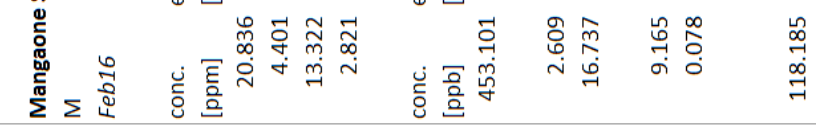

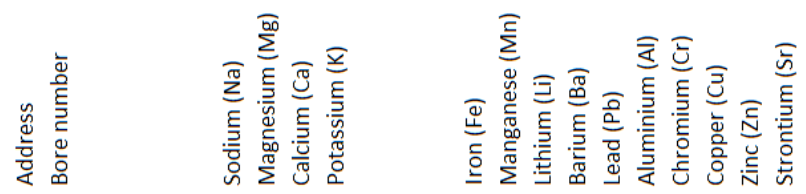




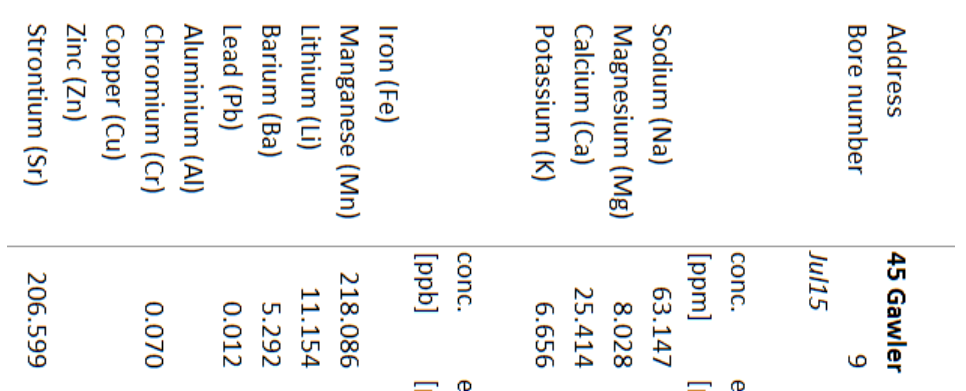

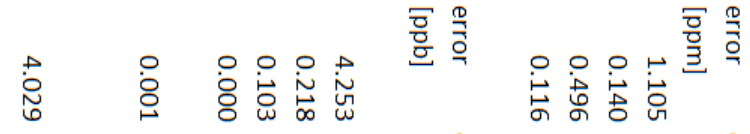

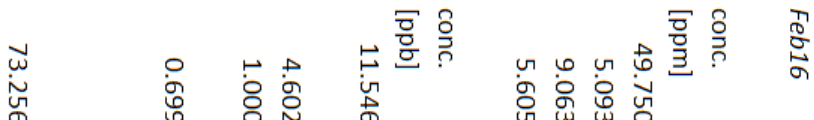

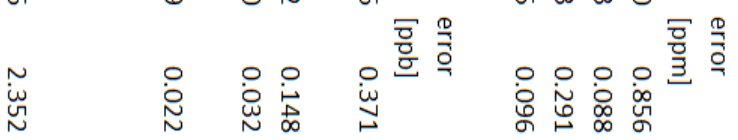

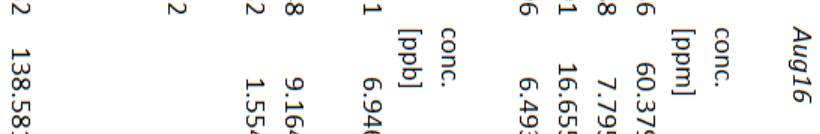

出

$\underset{\substack{b \\ ⿱ 亠 凶}}{\stackrel{b}{w}}$

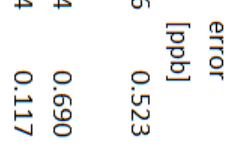

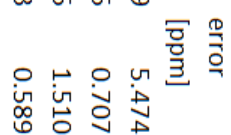

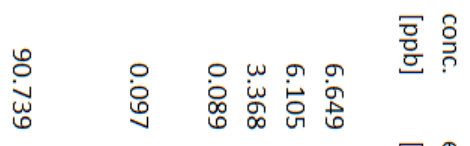

㟧

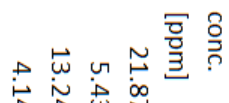

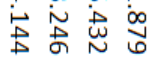

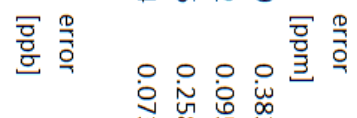

兽

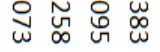

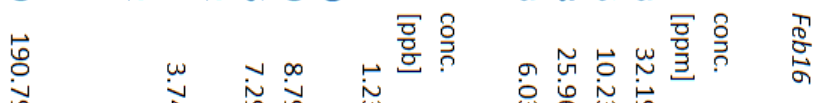

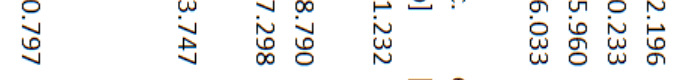

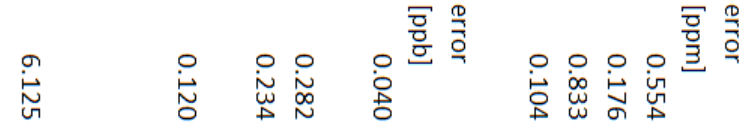

岕

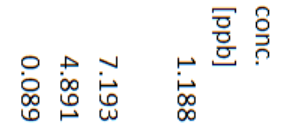

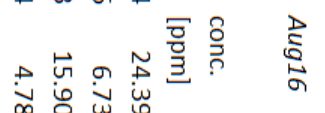

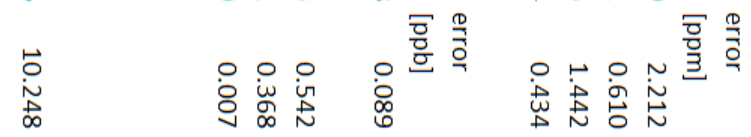




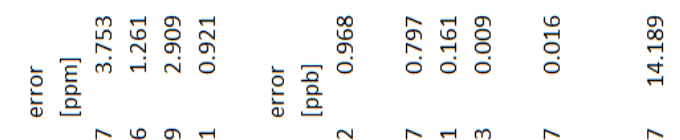

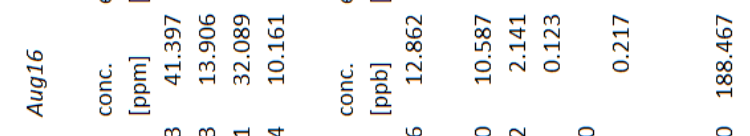

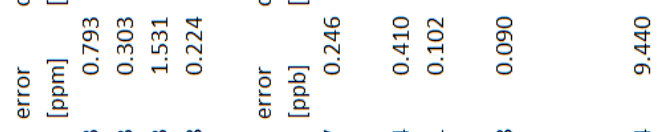

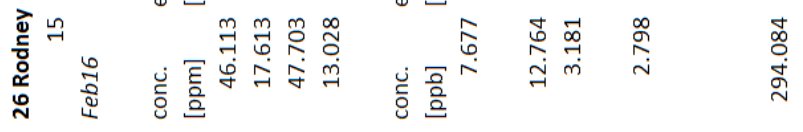

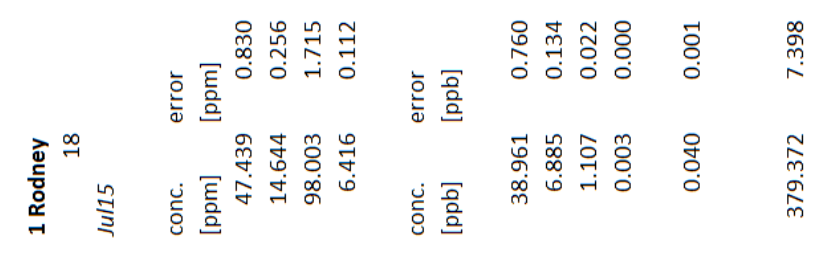

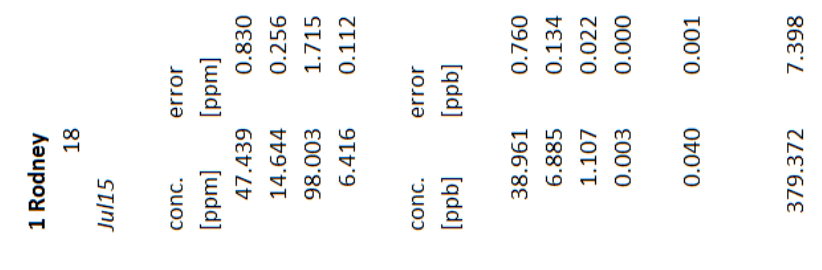

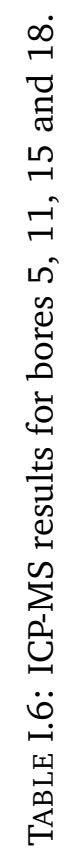
要

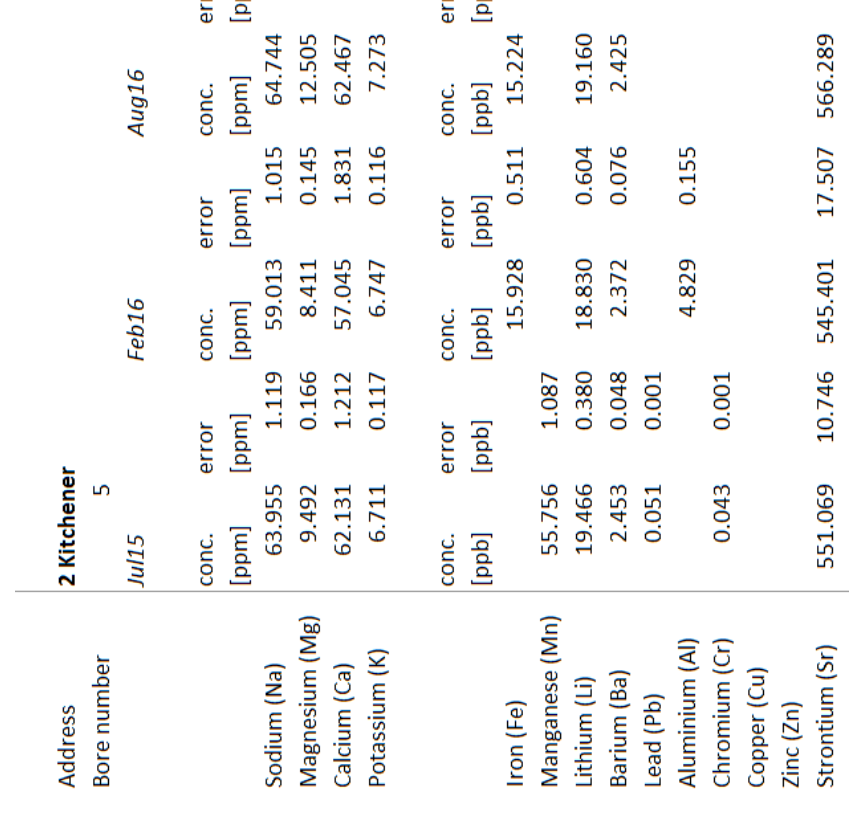




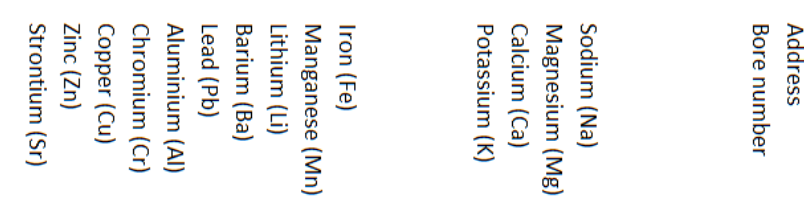

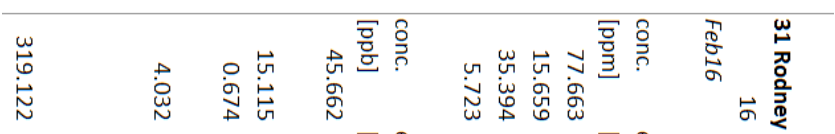

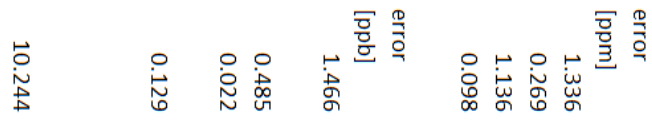

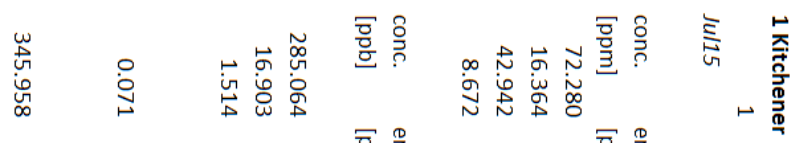

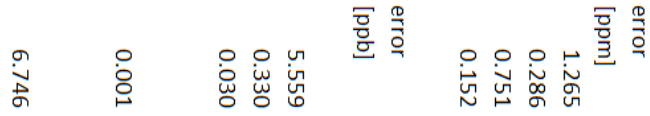

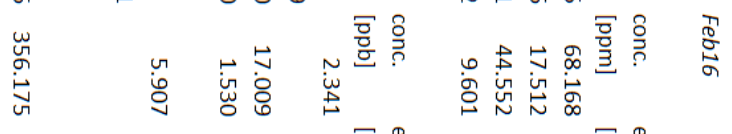

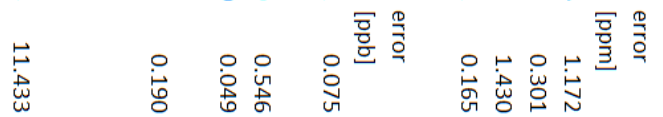

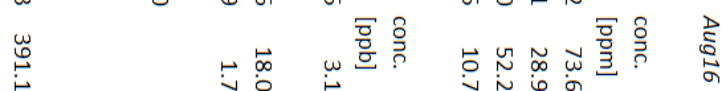

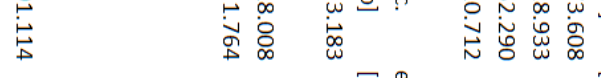

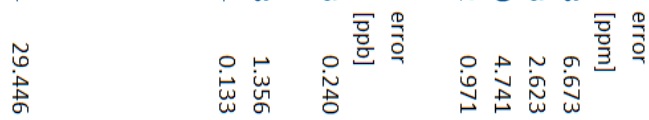

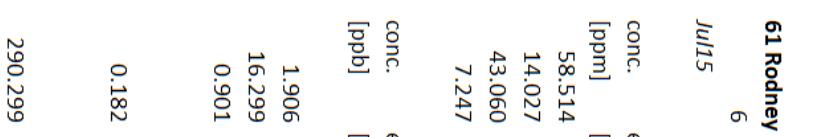

范

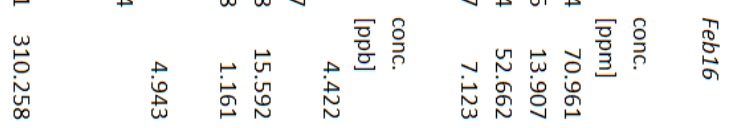

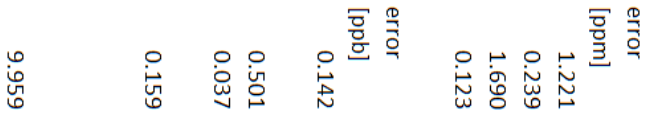

$\stackrel{2}{\stackrel{8}{6}}$

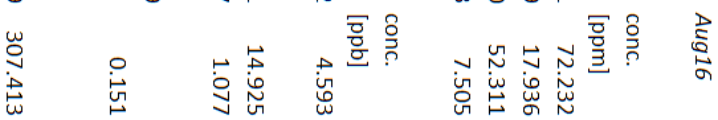

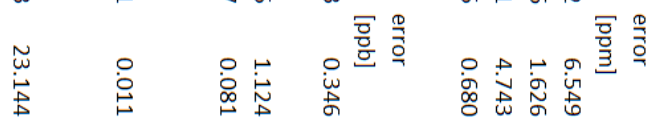

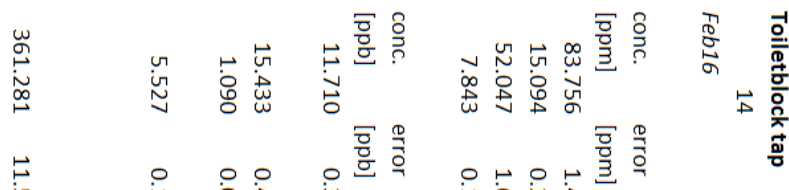

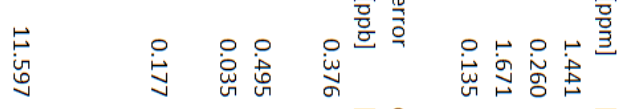

出 $\quad$ 이

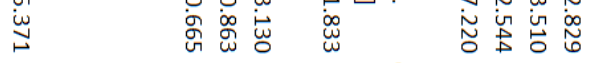

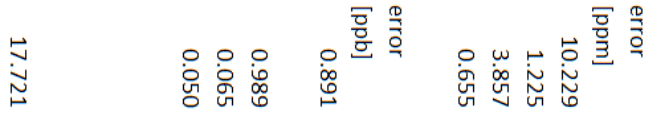




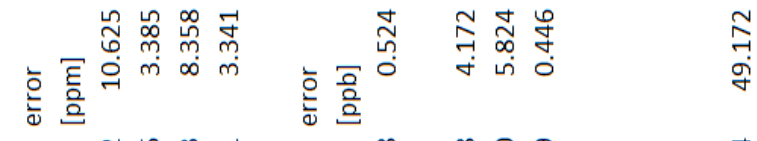

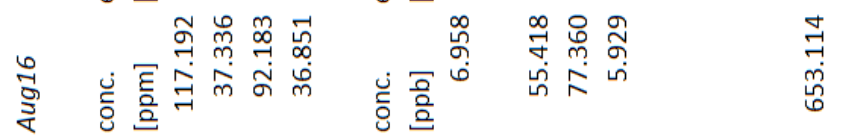

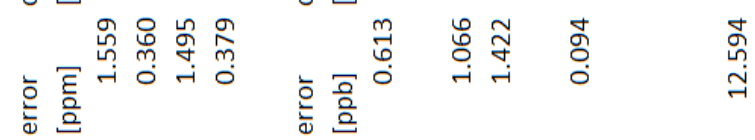

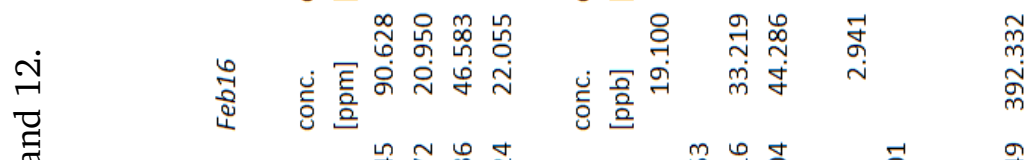

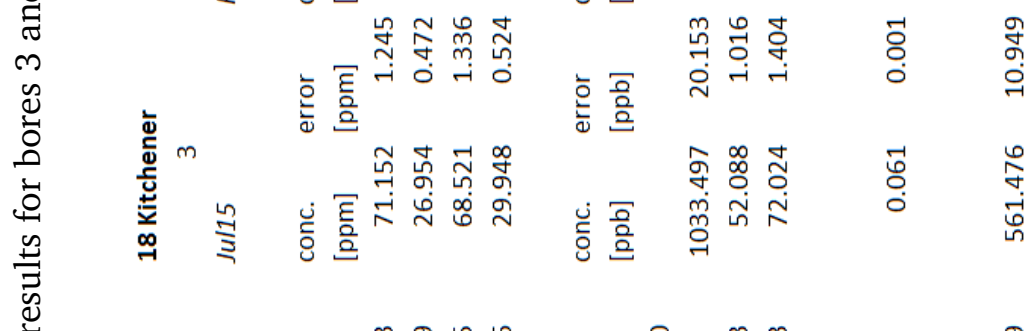

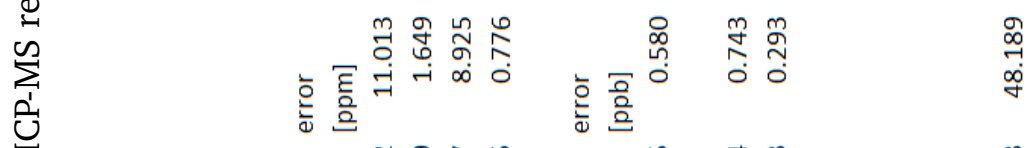

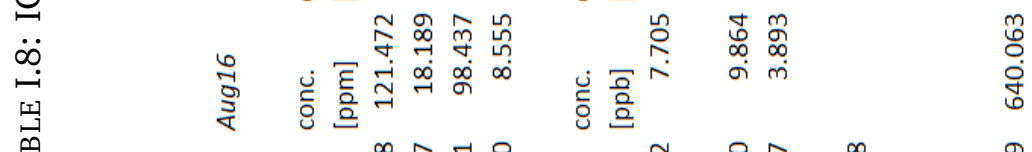

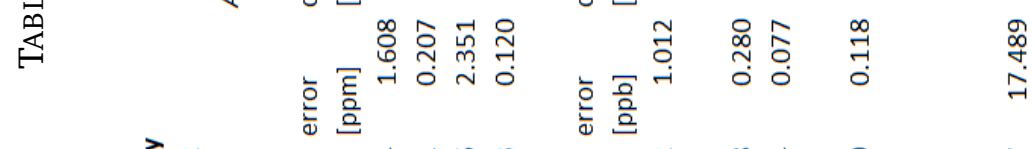

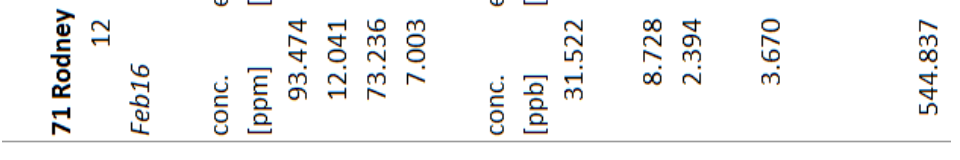

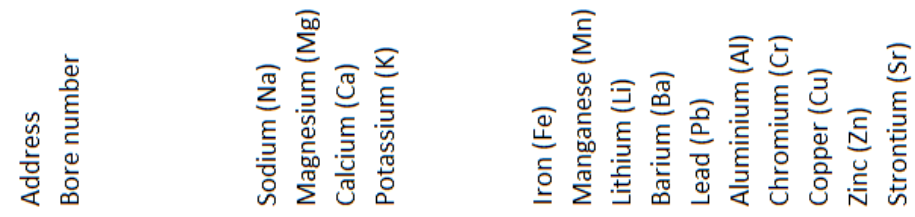




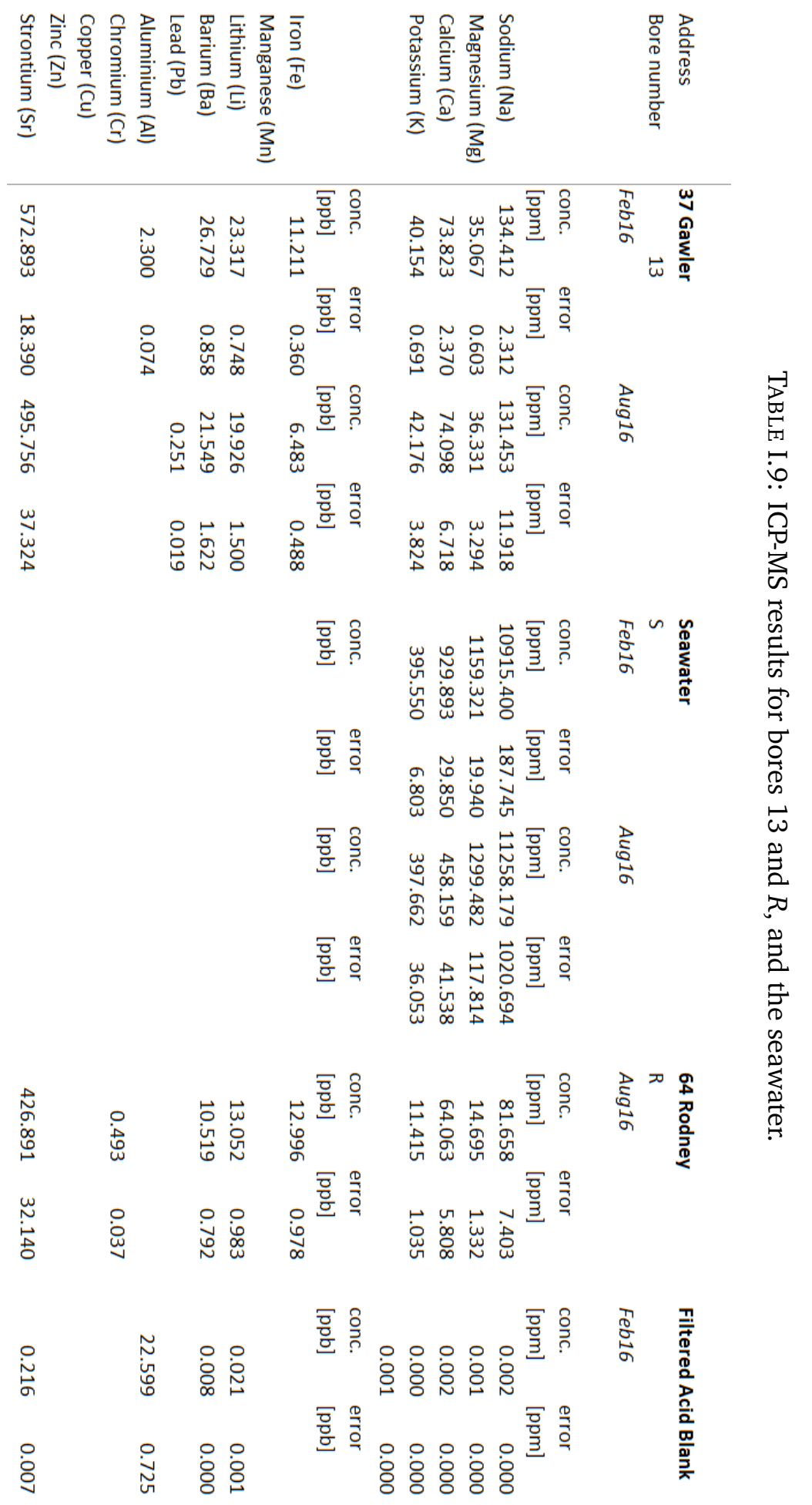

